\title{
3D dose verification for advanced radiotherapy
}

Citation for published version (APA):

van Elmpt, W. J. C. (2009). 3D dose verification for advanced radiotherapy. [Doctoral Thesis, Maastricht University]. Datawyse / Universitaire Pers Maastricht. https://doi.org/10.26481/dis.20090319we

Document status and date:

Published: 01/01/2009

DOI:

10.26481/dis.20090319we

Document Version:

Publisher's PDF, also known as Version of record

\section{Please check the document version of this publication:}

- A submitted manuscript is the version of the article upon submission and before peer-review. There can be important differences between the submitted version and the official published version of record.

People interested in the research are advised to contact the author for the final version of the publication, or visit the DOI to the publisher's website.

- The final author version and the galley proof are versions of the publication after peer review.

- The final published version features the final layout of the paper including the volume, issue and page numbers.

Link to publication

\footnotetext{
General rights rights.

- You may freely distribute the URL identifying the publication in the public portal. please follow below link for the End User Agreement:

www.umlib.nl/taverne-license

Take down policy

If you believe that this document breaches copyright please contact us at:

repository@maastrichtuniversity.nl

providing details and we will investigate your claim.
}

Copyright and moral rights for the publications made accessible in the public portal are retained by the authors and/or other copyright owners and it is a condition of accessing publications that users recognise and abide by the legal requirements associated with these

- Users may download and print one copy of any publication from the public portal for the purpose of private study or research.

- You may not further distribute the material or use it for any profit-making activity or commercial gain

If the publication is distributed under the terms of Article $25 \mathrm{fa}$ of the Dutch Copyright Act, indicated by the "Taverne" license above, 
3D dose verification for advanced radiotherapy

Wouter van Elmpt 


\section{Omslag}

"The 'flat shape' irritates me - I feel as if I were shouting to my figures, "You are too fictitious for me; you just lie there static and frozen together; do something, come out of there and show me what you are capable of!" So I make them come out of the plane." - M.C. Escher.

De cover laat zien dat M.C. Escher een meester was in het omtoveren van 2D objecten (in één vlak) naar 3D (in de ruimte) creaties, zoals weergegeven in de afbeelding op de omslag. De analogie met dit proefschrift is dat hierin ook een methode beschreven wordt die start met 2D informatie, gemeten vooraf of gedurende behandeling, en hieruit de afgegeven stralingsdosis in 3D in een patiënt bepaald kan worden.

\section{Copyright omslag}

M.C. Escher's “Magic Mirror" (c) 2008 The M.C. Escher Company B.V. - Baarn - Holland. Alle rechten voorbehouden.

(C) Copyright W.J.C. van Elmpt, Maastricht 2009

Druk: Datawyse | Universitaire Pers Maastricht

ISBN: $\quad 9789052788050$ 


\section{D dose verification for advanced radiotherapy}

\section{Proefschrift}

ter verkrijging van de graad van doctor aan de Universiteit Maastricht, op gezag van de Rector Magnificus prof. mr. G.P.M.F. Mols, volgens het besluit van het College van Decanen,

in het openbaar te verdedigen

op donderdag 19 maart 2009 om 14.00 uur

door

Wouter Johannes Catharina van Elmpt

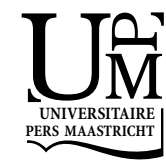




\section{Promotor}

Prof. dr. Ph. Lambin

\section{Copromotoren}

Dr. ir. A.L.A.J. Dekker

Dr. B.J. Mijnheer

\section{Beoordelingscommissie}

Prof. dr. J. Wildberger (voorzitter)

Prof. dr. B. Heijmen (Erasmus MC - Rotterdam)

Prof. dr. ir. B. ter Haar Romeny (Technische Universiteit Eindhoven / UM)

Dr. F. Verhaegen

Prof. dr. ir. C. De Wagter (Universiteit Gent)

The printing of this thesis was partially supported by Siemens Nederland NV. 


\section{Contents}

\section{Introduction}

Chapter 1 Introduction and outline of the thesis 9

Chapter 2 A literature review of electronic portal imaging for radiotherapy 17 dosimetry

\section{Pre-treatment dose verification}

Chapter 3 A Monte Carlo based three-dimensional dose reconstruction method derived from portal dose images

Chapter 4 Treatment verification in the presence of inhomogeneities using EPID-based three-dimensional dose reconstruction

Chapter 5 The next step in patient-specific QA: 3D dose verification of conformal and intensity-modulated RT based on EPID dosimetry and Monte Carlo dose calculation

Chapter 6 Transition from a simple to a more advanced dose calculation algorithm for radiotherapy of non-small cell lung cancer (NSCLC): Implications for clinical implementation in an individualized doseescalation protocol

\section{Treatment verification and in vivo dosimetry}

Chapter 7 Calibration of megavoltage cone-beam CT for radiotherapy dose calculations: Correction of cupping artifacts and conversion of CTnumbers to electron density

Chapter 8 3D in vivo dosimetry using megavoltage cone-beam CT and EPID dosimetry

\section{Discussion}

Chapter 9 General discussion and future perspectives

Summary

Samenvatting

List of publications

Dankwoord 

INTRODUCTION 



\section{CHAPTER}

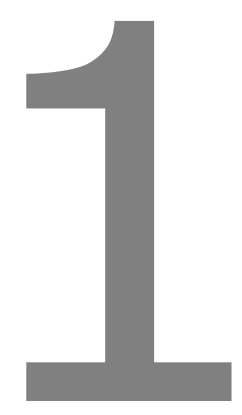

Introduction and outline of the thesis 



\section{INTRODUCTION}

Radiotherapy, together with surgery and chemotherapy, is one of the three main modalities for treating cancer. High energy beams of ionizing radiation of photons, electrons or ions, are used to destroy the tumor cells inside the human body. The aim of radiotherapy is to deliver a radiation dose to the tumor while sparing the normal tissue and possible nearby organs at risk. In external beam radiotherapy, a treatment plan is usually designed applying anatomical information based on a snapshot in time, i.e. the planning computed tomography (CT)-scan, made typically one or two weeks before the patient starts the treatment. In order to avoid damage of healthy tissue and adequate coverage of the tumor, accurate geometric and dosimetric design of the treatment plan used for the entire course of a treatment is necessary. Geometric accuracies of 2-3 $\mathrm{mm}$ are required ${ }^{1}$ for treatment plans with tight conformal dose distributions around the target volume and dosimetric differences of 5-7\% may already cause significant changes in tumor control or damage the healthy tissue. ${ }^{2,3}$ Radiotherapy is often performed in a fractioned schedule, where the total dose is delivered in multiple treatments, e.g. a typical fractionation scheme can consist of 30-40 fractions, 5 times per week. This is done to allow the healthy tissue to recover between the various fractions of treatment. Because of this fractionated schedule, it is important to maintain the same geometric and dosimetric accuracy across treatment sessions.

\section{Treatment verification}

Verification of the treatment plan and of the actual treatment delivery is of importance to prevent errors that can have enormous consequences in radiotherapy. Incidents such as in Panama ${ }^{4}$, Exeter (UK) ${ }^{5}$ and Costa Rica ${ }^{6}$ that happened $10-20$ years ago are known to most professionals involved in radiotherapy. But more recent accidents for instance in Epinal (France) $)^{7}$ and Glasgow $(U K)^{8}$, show that centers having modern equipment are also susceptible to incidents. Treatment verification is not only needed to avoid accidents, but quality assurance and control programs are also necessary to maintain the high level of accuracy required in a radiotherapy department. Verification procedures implemented prior to treatment or by applying in vivo dosimetry, are necessary to prevent or, in an early stage, detect errors and prevent accidents. The type of verification procedure determines which type of error and the magnitude of the error that can be detected.

However, if all treatment parameters are correct and the delivery process is as planned from a technical point of view, this will not automatically mean that the treatment in a clinical setting is delivered as optimal as possible. The dose distribution calculated using the planning CT scan serves as a reference throughout the entire course of radiotherapy. Changes in patient anatomy between planning CT scan and start of treatment, or occurring at a later stage which is sometimes induced by the effect of radiation, might affect the validity of this assumption and decrease the optimal result of the intended treatment. A fractionated course of radiotherapy may take up to several weeks, and previous studies have shown that differences during treatment do occur. For example in lung cancer patients, a decrease of tumor size ${ }^{9,10}$ 
and a drift of the mean position of the tumor ${ }^{11}$ may occur. During prostate cancer irradiation, organ motion results in changes of the prostate, bladder and rectum position between the various fractions of treatment. ${ }^{12,13}$ Furthermore, weight loss and shrinking of the tumor volume are frequently observed phenomena during a series of treatments of head-and-neck cancer patients and may result in a shift of the position of organs at risk, e.g. the parotids or spinal cord, towards the high dose regions. ${ }^{14,15}$

\section{Treatment adaptation}

To take these anatomy changes during treatment into account, a continuous adaptation of an individualized treatment plan has been suggested in recent years. This process is commonly referred to as adaptive radiotherapy (ART) ${ }^{16}$ : a feedback loop is introduced in the workflow of radiotherapy that incorporates information gathered during the course of treatment and updates, i.e. adapts, the treatment. ART is usually performed based on multiple image data-sets acquired during treatment, either using repeated conventional CT scans or by applying the recent developments in in-room CT imaging, e.g. kV or MV (cone-beam) CT scanning. Nowadays, images form the basis of this feedback loop, i.e. image-guided radiotherapy $(\text { IGRT })^{17,18}$, but the next step to extend the possibilities of ART is to add information about the dose delivered to the patient in this workflow. Then, ART will incorporate not only the actual patient anatomy during treatment but also the actual dose distribution in a changing anatomy. The entire chain of radiotherapy, including the dose delivery to the patient, is then part of this adaptive workflow and ART has evolved from image-guided radiotherapy towards dose-guided radiotherapy (DGRT): decisions and treatment adaptation based on the delivered dose distribution.

\section{OBJECTIVE OF THE STUDY}

The main objective of this study is to investigate how information of the dose delivery, either prior to or during treatment, can be used to derive information on the threedimensional (3D) dose distribution inside the patient. For this purpose, models will be developed for the verification of the 3D dose distribution, both pre-treatment and during treatment, based on the actual patient geometry and energy fluence delivered by an accelerator. These models are pre-requisites to facilitate the extension of the feedback loop of adaptive radiotherapy from image-guided radiotherapy towards true dose-guided radiotherapy.

\section{OUTLINE OF THE THESIS}

In this Chapter 1, a general introduction is given on the background and importance of dose verification procedures in a radiotherapy department, with a focus on the use of $3 \mathrm{D}$ dose verification for adaptive and dose-guided radiotherapy. A more elaborate 
overview on the use of electronic portal imaging devices (EPIDs) for (pre-)treatment dosimetry is presented in Chapter 2, where a review of published methods of performing dosimetry with EPIDs is given. This review focuses on the dosimetric characteristics of EPIDs, the various ways of calibrating an EPID for dosimetry and application of the EPID for transmission based and non-transmission based verification methods, either pre-treatment or during treatment. Finally in this chapter, some discussion is dedicated to the type of errors that can be detected with EPID dosimetry and on frequently used acceptance and rejection criteria for interpreting the differences observed between planned and measured dose distributions. Also, current clinical applications of EPID dosimetry are summarized.

Three-dimensional dose verification strategies are applied in two different parts of the treatment procedure. A 3D verification strategy prior to treatment (pre-treatment dose verification) and a 3D verification strategy applied during treatment (in vivo dosimetry). This thesis deals with both strategies.

First, the pre-treatment verification part deals with verification methods and procedures that are applied before the first fraction of a treatment is given. These pretreatment verification strategies are useful for detecting errors made during treatment planning, dose calculation, treatment plan transfer or for detecting differences between planned and actual delivered energy fluence distributions of treatment fields. In Chapter 3 a method is described that measures the dose delivered by the linear accelerator with an EPID and reconstructs the 3D dose distribution inside a predefined phantom geometry using a Monte Carlo dose calculation. In this chapter, the model is described in detail and evaluated for homogeneous phantoms. Chapter 4 describes an extension of the model to situations in which tissue inhomogeneities are present. The extended model has been verified using inhomogeneous phantoms of various dimensions, including anthropomorphic phantoms. Because the method is able to reconstruct a 3D dose distribution, a comparison with the 3D dose distribution calculated using the clinically applied treatment planning system is also performed. In Chapter $\mathbf{5}$ the application of the methods discussed in Chapters 3 and 4 in a clinical setting will be described, as well as an evaluation of the actual patient treatment plans with the dose reconstructed inside the planning CT scan. Two treatment sites have been investigated. For the first site, the thorax region, treatment plans have been calculated using the convolution algorithm of the treatment planning system and the more advanced superposition algorithm, and next compared with the 3D reconstructed dose distribution based on measured EPID images and Monte Carlo simulations. For the second treatment site, the head-and-neck region, for patients treated with intensitymodulated radiotherapy (IMRT), a comparison between the calculated dose of the treatment planning system using the superposition algorithm and the 3D reconstructed dose has been made. The various dose distributions are evaluated by comparing dose-volume histograms (DVHs). Chapter 6 focuses on the importance of using accurate dose calculation algorithms for designing 3D conformal treatment plans for patients diagnosed with non-small cell lung cancer. Different treatment plans are designed using both the simple convolution and the more advanced superposition algorithm following a dose-escalation protocol based on normal tissue constraints. The various plans calculated using different dose calculation algorithms are compared in terms of dose in the target volume and normal tissue dose, and the implications for 
implementation in clinical routine are discussed. To evaluate the accuracy of the calculated dose of both the simple and the more advanced dose calculation algorithm, the 3D dose reconstruction method described in Chapters 3-5 was used to verify the dose in a subset of the patients.

The second part of this thesis deals with verification procedures that are applied during treatment. For this purpose, it is necessary to have an accurate model of the patient anatomy prior to, or just after the treatment, while the patient is still in the treatment position. Chapter 7 describes a method to acquire a megavoltage conebeam CT scan in the treatment room and a calibration procedure to use these images for dose calculation purposes. The images are corrected for the cupping artifact and the Hounsfield units are converted into electron density values. This makes these images suitable for dose calculation using the treatment planning system or using Monte Carlo dose calculations. With images of the actual patient anatomy during treatment, the imaging part necessary to perform dose-guided radiotherapy is available. Chapter 8 combines the methods developed for the pre-treatment dose-guided verification procedures with on-line MV cone-beam CT imaging. The actual delivered treatment fields are captured behind the patient using the EPID and these fields are used in combination with the on-line acquired cone-beam CT scan. The model backprojects the transmission EPID images behind a patient during treatment to reconstruct the 3D dose delivered of that fraction inside the actual patient anatomy acquired using the MV cone-beam CT. In this chapter, the model is described and validated using a phantom study. Clinical applicability is shown for a head-and-neck cancer patient treated with IMRT.

Chapter 9 includes a discussion of the various methods for dose verification described in this thesis and a general discussion on the use of 3D pre-treatment verification and 3D in vivo dosimetry methods for dose-guided radiotherapy. An outlook is given on how to incorporate these advanced models in an adaptive way during the course of a radiotherapeutic treatment.

\section{REFERENCES}

1J.R. Palta, S. Kim, J.G. Li, and C. Liu, Intensity-Modulated Radiation Therapy. The State of the Art, in Medical Physics Monograph No. 29, J.R. Palta and T.R. Mackie, Editors. 2003, Medical Physics Publishing: Madison, WI. p. 495-514.

${ }^{2} \mathrm{~A}$. Brahme, "Dosimetric precision requirements in radiation therapy," Acta Radiol Oncol 23, 379-391 (1984).

${ }^{3}$ B.J. Mijnheer, J.J. Battermann, and A. Wambersie, "What degree of accuracy is required and can be achieved in photon and neutron therapy?," Radiother Oncol 8, 237-252 (1987).

${ }^{4}$ S. Vatnitsky, P. Ortiz Lopez, J. Izewska, A. Meghzifene, and V. Levin, "The radiation overexposure of radiotherapy patients in Panama 15 June 2001," Radiother Oncol 60, 237-238 (2001).

5J.S. Tobias, "What went wrong at Exeter?," BMJ 297, 372-373 (1988).

${ }^{6}$ International Atomic Energy Agency (IAEA), Accidental Overexposure of Radiotherapy Patients in San José, Costa Rica. Special publication series. 1998, Vienna.

${ }^{7}$ D. Ash, "Lessons from Epinal," Clin Oncol 19, 614-615 (2007).

${ }^{8}$ M.V. Williams, "Radiotherapy near misses, incidents and errors: radiotherapy incident at Glasgow," Clin Oncol 19, 1-3 (2007). 
${ }^{9}$ P.A. Kupelian, C. Ramsey, S.L. Meeks, T.R. Willoughby, A. Forbes, T.H. Wagner, and K.M. Langen, "Serial megavoltage $\mathrm{CT}$ imaging during external beam radiotherapy for non-small-cell lung cancer: observations on tumor regression during treatment," Int J Radiat Oncol Biol Phys 63, 1024-1028 (2005).

${ }^{10}$ G. Bosmans, A. van Baardwijk, A. Dekker, M. Ollers, L. Boersma, A. Minken, P. Lambin, and D. De Ruysscher, "Intra-patient variability of tumor volume and tumor motion during conventionally fractionated radiotherapy for locally advanced non-small-cell lung cancer: a prospective clinical study," Int J Radiat Oncol Biol Phys 66, 748-753 (2006).

${ }^{11}$ J.J. Sonke, J. Lebesque, and M. van Herk, "Variability of four-dimensional computed tomography patient models," Int J Radiat Oncol Biol Phys 70, 590-598 (2008).

${ }^{12}$ J.C. Roeske, J.D. Forman, C.F. Mesina, T. He, C.A. Pelizzari, E. Fontenla, S. Vijayakumar, and G.T. Chen, "Evaluation of changes in the size and location of the prostate, seminal vesicles, bladder, and rectum during a course of external beam radiation therapy," Int J Radiat Oncol Biol Phys 33, 1321-1329 (1995).

${ }^{13}$ J.M. Balter, H.M. Sandler, K. Lam, R.L. Bree, A.S. Lichter, and R.K. ten Haken, "Measurement of prostate movement over the course of routine radiotherapy using implanted markers," Int J Radiat Oncol Biol Phys 31, 113-118 (1995).

${ }^{14}$ J.L. Barker, Jr., A.S. Garden, K.K. Ang, J.C. O'Daniel, H. Wang, L.E. Court, W.H. Morrison, D.I. Rosenthal, K.S. Chao, S.L. Tucker, R. Mohan, and L. Dong, "Quantification of volumetric and geometric changes occurring during fractionated radiotherapy for head-and-neck cancer using an integrated CT/linear accelerator system," Int J Radiat Oncol Biol Phys 59, 960-970 (2004).

${ }^{15}$ E.K. Hansen, M.K. Bucci, J.M. Quivey, V. Weinberg, and P. Xia, "Repeat CT imaging and replanning during the course of IMRT for head-and-neck cancer," Int J Radiat Oncol Biol Phys 64, 355-362 (2006).

${ }^{16}$ D. Yan, F. Vicini, J. Wong, and A. Martinez, "Adaptive radiation therapy," Phys Med Biol 42, 123-132 (1997).

${ }^{17}$ L.A. Dawson and M.B. Sharpe, "Image-guided radiotherapy: rationale, benefits, and limitations," Lancet Oncol 7, 848-858 (2006).

${ }^{18}$ D. Verellen, M.D. Ridder, N. Linthout, K. Tournel, G. Soete, and G. Storme, "Innovations in image-guided radiotherapy," Nat Rev Cancer 7, 949-960 (2007). 



\section{CHAPTER}

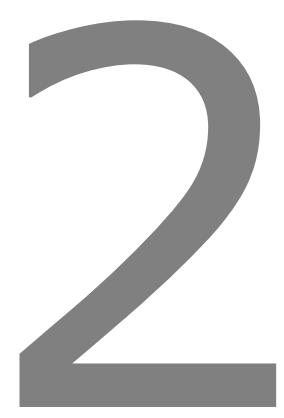

A literature review of electronic portal imaging
for radiotherapy dosimetry

Wouter van Elmpt, Leah McDermott, Sebastiaan Nijsten, Markus Wendling, Philippe Lambin and Ben Mijnheer

Published in: Radiotherapy and Oncology 88(3):289-309, 2008. 


\begin{abstract}
Electronic portal imaging devices (EPIDs) have been the preferred tools for verification of patient positioning for radiotherapy in recent decades. Since EPID images contain dose information, many groups have investigated their use for radiotherapy dose measurement. With the introduction of the amorphous silicon EPIDs, the interest in EPID dosimetry has been accelerated because of the favourable characteristics such as fast image acquisition, high resolution, digital format, and potential for in vivo measurements and $3 \mathrm{D}$ dose verification. As a result, the number of publications dealing with EPID dosimetry has increased considerably over the past 15 years. The purpose of this paper was to review the information provided in these publications. Information available in the literature included dosimetric characteristics and calibration procedures of various types of EPIDs, strategies to use EPIDs for dose verification, clinical approaches to EPID dosimetry, ranging from point dose to full 3D dose distribution verification, and current clinical experience. Quality control of a linear accelerator, pretreatment dose verification and in vivo dosimetry using EPIDs are now routinely used in a growing number of clinics. The use of EPIDs for dosimetry purposes has matured and is now a reliable and accurate dose verification method that can be used in a large number of situations. Methods to integrate 3D in vivo dosimetry and image-guided radiotherapy (IGRT) procedures, such as the use of $\mathrm{kV}$ or $\mathrm{MV}$ cone-beam $\mathrm{CT}$, are under development. It has been shown that EPID dosimetry can play an integral role in the total chain of verification procedures that are implemented in a radiotherapy department. It provides a safety net for simple to advanced treatments, as well as a full account of the dose delivered. Despite these favourable characteristics and the vast range of publications on the subject, there is still a lack of commercially available solutions for EPID dosimetry. As strategies evolve and commercial products become available, EPID dosimetry has the potential to become an accurate and efficient means of large-scale patient-specific IMRT dose verification for any radiotherapy department.
\end{abstract}




\section{INTRODUCTION}

\section{Motivation}

Advances in external beam radiotherapy have increased the accuracy requirements for dose delivery to patients. Quality control procedures before and during treatment can potentially ensure a high level of accuracy necessary for treatments designed to achieve adequate tumour control and reduction of normal tissue complications. As a result of the high number of patients being treated in radiotherapy departments world-wide, there has to be a trade-off between the extent of verification required to attain a certain level of accuracy and the work-load required to carry out such procedures.

In general, there are several aspects that should be incorporated in radiotherapy verification procedures. These include procedural verification using well-documented protocols and workflow, set-up verification measurements of the patient position and also verification of the dose delivered to the patient. Documentation and workflow are highly dependent on the size of the department and a myriad other departmentspecific factors. Set-up verification is gaining significant ground in the field of externalbeam radiotherapy with the recent proliferation of 3D and 4D (in-room) imaging tools. Detailed reviews on the clinical use of electronic portal imaging devices (EPIDs) for 2D portal imaging have been provided by Herman et al. $^{1-3}$, Langmack $^{4}$ and Antonuk ${ }^{5}$. Standards in dose verification also need to keep up with the pace, since high levels of geometrical accuracy and other QA procedures become meaningless when the dose cannot be delivered as planned.

With the increased use of intensity-modulated radiotherapy (IMRT), higher dose prescriptions and changes to electronic recording and transfer of patient treatment data, the demand for patient-specific verification has increased. The complexity of treatment plans is also increasing, making it more difficult to discover possible errors because the dose levels and field shapes are no longer intuitive for specific treatment sites, and are therefore more difficult to detect by experience. During the treatment planning process, deviations from the planned dose can be present if characteristics of the linear accelerator are not modelled accurately in the treatment planning system (TPS), e.g. parameters such as the tongue-and-groove effect or multi-leaf collimator (MLC) transmission. Errors can also occur due to data transfer problems, especially when converting from paper to electronic systems, or from manual to automatic systems. Regardless of whether the treatment technique is conventional or advanced, the department is large or small, or the treatment makes use of the latest technology or not, errors can, and often do, occur during the delivery process. Recommended QA procedures might no longer be sufficient and patient-specific verification procedures are therefore necessary to detect possible errors for these complex plans. Furthermore, the legal requirements for radiotherapy are increasing. National or international legislation (e.g. the EC Directive 97/43) impose requirements on these types of verification procedures.

Not long after their clinical introduction for set-up verification it was realised that EPID images contain dose information. Consequently, several groups investigated the dosimetric characteristics of various types of EPIDs. In some cases, EPIDs have gone on 
to replace traditional dosimetry devices in the clinic for plan verification. Interest in EPID dosimetry has been accelerated by the advantages of fast image acquisition, high resolution, digital format, and potential for in vivo measurements and 3D dose verification. Figure 2.1 illustrates this increased interest through the number of publications in peer-reviewed journals relating to EPID dosimetry over a period of more than a decade since its inception.

The aim of this study was to review how EPIDs have contributed to the verification of the dose delivered either prior to or during the treatment of a patient. The dosimetric characteristics published for different types of EPIDs are first summarised, followed by the various strategies to implement EPID dosimetry in the clinic. Finally, a review of current clinical practice is presented with special attention to acceptance and rejection criteria used in various institutes.

\section{Search strategy}

A comprehensive search strategy through the indexed database 'Pubmed' was performed using search terms that included "EPID", "portal dosimetry", "portal imaging", "dose verification", "quality control", "quality assurance", "treatment verification", "pre-treatment verification" and "in vivo dosimetry". Relevant papers, including relevant cited references in these papers, published in English between 1985 and January 2008 were included.

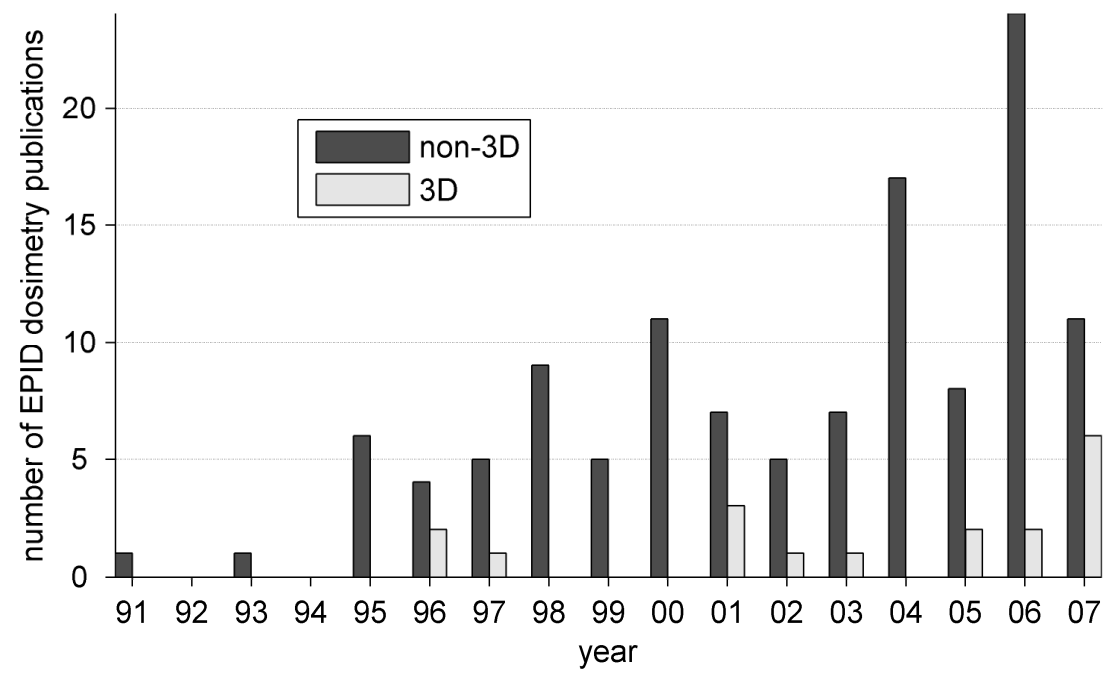

Figure 2.1: Number of publications on EPID dosimetry (as found on Pubmed www.pubmed.com). 


\section{DEVICES}

\section{Dose verification devices}

This review will focus primarily on the use of EPIDs for dosimetry, other devices are also used for verifying the dose either before or during treatment. Devices such as point detectors, film and gel have been widely reported. More detailed information about the various systems used for verification of IMRT can be found elsewhere, e.g. ${ }^{6,7}$.

Like EPIDs, various matrix detectors have been produced for measuring energy fluence or absorbed dose in two dimensions. ${ }^{7,8}$ Commercial options are available based on different measurement techniques. These include two-dimensional detectors consisting of a large number of ionisation chambers ${ }^{9,10}$ or diodes ${ }^{11,12}$ placed in a regularly spaced array or at specific points in a phantom. The use of fluorescent screens in combination with an optical camera system has also been used for dose measurements. ${ }^{13-15}$ Matrix detectors can be attached to the gantry of the linear accelerator or placed on the treatment couch. A quick verification of the beam is performed by comparing the output of these devices with a dose distribution predicted by the TPS. A possible drawback of these devices is that they have relatively few measuring points and therefore a low spatial resolution. This potentially limits the procedures for verification of highly intensity-modulated fields. A possible solution has been proposed whereby the detector is shifted between multiple irradiations of the same plan, which extends the verification time. ${ }^{10}$

An advantage of using EPIDs for dosimetry is that most departments wishing to deliver, and hence verify, more complicated treatments usually already have one attached to their linac. Using this device for dosimetry usually only requires additional software. The various EPIDs that are commercially available can be categorized according to their technical design. The first commercially available EPIDs were the liquidfilled ionisation chamber EPID and the camera-based EPID. More recently, the amorphous-silicon EPID has become the most commonly available portal imager. For a detailed overview of the historical perspective and development of EPID technology, as well as more in depth information on the various types of EPIDs that have been produced, see the review articles by Boyer et al. ${ }^{16}$, Antonuk ${ }^{5}$ and more recently by Kirby and Glendinning ${ }^{17}$.

\section{EPIDs used for dosimetry}

One of the first publications that proposed to use EPIDs for dose measurement involved the scanning liquid-filled ionisation chamber EPID (Li-Fi EPID, also referred to as 'SLIC EPID' in the literature). ${ }^{18}$ It was developed in the late 1980s/early 1990s at the Netherlands Cancer Institute, Amsterdam by Meertens and van Herk ${ }^{19-21}$ and became commercially available as PortalVision (Varian, Palo Alto, California, USA). Original versions consisted of a matrix of $256 \times 256$ ionisation chambers filled with an organic liquid (isooctane) over an area of $32 \times 32 \mathrm{~cm}^{2}$. Using the standard acquisition mode, the sampling time for one row was $20 \mathrm{~ms}$ with an image scan time of $5.6 \mathrm{~s}^{22}$ Due the relatively long read-out time, this device cannot measure dose directly, but is suitable for measuring dose-rate. The measured dose-rate can be converted to absolute dose 
by recording a continuous readout of the monitor chamber signal of the linac during image acquisition and the number of MUs delivered for the measured dose image. Dose-response characteristics of the Li-Fi EPID have been investigated by several groups. ${ }^{18,22-36}$ These characteristics can be summarised as follows: (i) the stability of the response is within $1 \%$ up to 2 years, as long as corrections for room temperature dependence and radiation damage are incorporated; (ii) the dose-rate/response relationship can be accurately described by an equation with a term proportional to the square-root of the dose-rate and another term linear to the dose-rate, or a powerlaw; (iii) if a mini-phantom is used to calibrate the system, additional corrections are required to account for lateral scatter within the EPID, which can be done via a deconvolution filter; (iv) a relative sensitivity map is needed to account for variation in response for each ionisation chamber-electrometer combination. The amount of buildup material required for electron equilibrium was investigated by Boellaard et al. ${ }^{26}$ They found that above the detector layer, the EPID had a water-equivalent thickness of $8 \mathrm{~mm}$. An additional $28 \mathrm{~mm}$ of polystyrene was required for a $25 \mathrm{MV}$ beam; however the disadvantage was that $4.5 \mathrm{~kg}$ was added to the mass of the EPID system and some deterioration in image quality was reported.

The scintillation crystal-photodiode detector, 'RTIMAGE', was also developed in the beginning of the 1990s and its use for dose measurements has been described in very early EPID dosimetry publications. It is a linear scanning array imager, developed at the Royal Marsden Hospital in London by Morton et al. ${ }^{37}$ A similar prototype was described by Symonds-Taylor et al. ${ }^{38}$ While not developed commercially, Hansen et al. $^{39}$ used the RTIMAGE to perform transit dosimetry and demonstrated the linear relationship between the response and dose, pulse height and pulse frequency. Mosleh-Shirazi et al. ${ }^{40}$ described a $2 \mathrm{D}$ crystal array mirror system that was used to measure transmission dose images.

The camera-based EPID was developed into a commercial product two decades ago. This device consists of a fluorescent phosphor-screen with a metal plate on top that converts high-energy photons into visible photons. These photons are imaged with a video camera (mostly CCD-based cameras are used) by means of mirrors and a lens. A large portion of the field can be imaged quickly due to the fast read-out of the camera and it has a high spatial resolution. The various camera-based systems for which the dosimetric properties are described are SRI-100 ${ }^{41-43}$ (Philips Medical Systems, Best, The Netherlands), iView ${ }^{44}$ (Elekta Oncology Systems, Crawley, UK), TheraView $\mathrm{NT}^{45-48}$ (Cablon, Leusden, The Netherlands). The measured grey scale value is approximately linear with dose and does not depend on the absorber thickness that is placed in the beam. Some groups have introduced a correction function to correct for the non-linearity of the EPID electronics. ${ }^{43,45}$ There is, however, a large field size dependence caused by scattered visible photons inside the optical system. This dependence is usually removed via deconvolution with a cross-talk kernel that describes the spread of the photons inside the optical system ${ }^{42,43}$, or using position specific cross-talk contributions $^{48,49}$, while also anti-scatter grids have been proposed ${ }^{50,51}$. In addition a sensitivity matrix is used to remove some of the residual cross-talk and to correct for possible non-uniformities in the phosphor-layer. The short-term variation in the response is smaller than $1 \%(1 \mathrm{SD})$ and its long-term stability is within $1 \%-2 \%$ (1 SD) for periods up to one year. ${ }^{42,45,47,52,53}$ Once the camera-based EPID is calibrated, mainly by 
removing the influence of the optical scatter effects, it is a suitable device for performing portal dosimetry with deviations around 1\%-2\% (1 SD).

The most common type of EPID available today is the amorphous-silicon EPID (a-Si EPID) or flat-panel imager. It was first described by Antonuk et al. ${ }^{54,55}$ from the University of Michigan Medical Center, Ann Arbor, USA. The panel consists of an x-ray converter, light detector, and an electronic acquisition system for receiving and processing the resulting digital image. The dose-response behaviour of the three commercially available a-Si EPIDs has been described, including the Elekta iView GT system ${ }^{56-60}$, the Siemens OptiVue ${ }^{61,62}$ and the Varian aSi PortalVision aS500/aS1000 ${ }^{63-70}$. Stability of the response has been reported as $0.5 \%$ ( $1 \mathrm{SD}$ ) over 2 years for panels fixed to linacs with lower energy beams (4 and $6 \mathrm{MV}$ photon beams). ${ }^{71}$ The dose-response relationship is independent of the dose-rate and approximately linear with integrated dose.

Over-sensitivity to low energies has also been reported, where the signal is influenced by the scatter within the bulk layers of the imager. ${ }^{15,67,70,73,74}$ The EPID response is therefore dependent on the off-axis position of a specific pixel (beam softening) as well as the thickness of the phantom or patient in the beam, which causes hardening of the photon beam, as reported by several groups. ${ }^{58,62,63,66,75}$ Various studies have used models to reduce the field size dependence from $5-8 \%$ (1 SD) to values less than $1-2 \%$ (1 SD). ${ }^{64,69,76}$ Yeboah et al. identified the effect of a copper plate on the attenuation of low-energy scattered photons, while producing additional annihilation, bremsstrahlung and scattered photons. Consequently, several groups have added an additional $\mathrm{Cu}$ plate on the detector (usually fixed within the housing under the cover). ${ }^{56,60,66,73,77}$ Additional build-up material for portal dosimetry serves to attenuate scattered radiation from the patient, to which the a-Si EPID is over-sensitive, and to ensure measurements are made beyond the position of dose maximum.

An additional issue specific to amorphous silicon EPIDs is that of ghosting and image lag. Both are due to the charge trapped within the photodiode layer resulting in both gain and offset effects. Ghosting is a gain effect whereby the trapped charge alters the electric field strength of the bulk and surface layers, lasting minutes and affecting subsequent images. Image lag is an offset effect as a result of incomplete charge transfer from the pixel capacitor. This trapped charge is read-out in subsequent frames, lasting up to $30 \mathrm{~s}$. Under-dosages from 4 to $10 \%$ at short irradiation time (2-5 MUs) relative to that of 1000 MUs have been reported for all commercially available aSi EPIDs. ${ }^{78}$ Ghosting was found to depend on irradiation time and the dose per frame, not on dose or dose-rate directly. ${ }^{56,73}$

Changes in EPID technology over recent decades have not been driven by the technical demands of dosimetry, but by the need for improved imaging standards for patient positioning, such as enhancement in image quality and faster read-out. Coincidentally, they have also aided in improving the EPID's capacity as a dosimeter. Faster read-out time has meant images can be used to measure integrated dose, instead of dose-rate (as with the Li-Fi EPID). Higher resolution, especially of the aSi EPID, has improved the resolution to which dose can be verified, along with the image quality as intended. Changes arising from EPID dosimetry requirements involve software changes, such as the PortalVision system by Varian. The earlier version (v6.1.03) used a reset every 64 frames to move the frame buffer content to the CPU, creating a dead time of $0.28 \mathrm{~s}$, or loss of one to two image frames (depending on the frame rate). This 
was a problem for dosimetry and corrected in subsequent versions of the software. ${ }^{65,78}$ As mentioned earlier, the addition of build-up material (e.g. a copper plate) is important for dosimetry and may be incorporated in future EPID designs. At this stage both imaging and dosimetry functions would have to be considered by manufacturers, and as in the past, whichever ends up becoming the primary function will drive technological advances.

An overview of the various types of EPIDs and key references to the literature are given in Table 2.1.

Table 2.1: List of key references that describe dosimetric properties and calibration methods for various EPIDs.

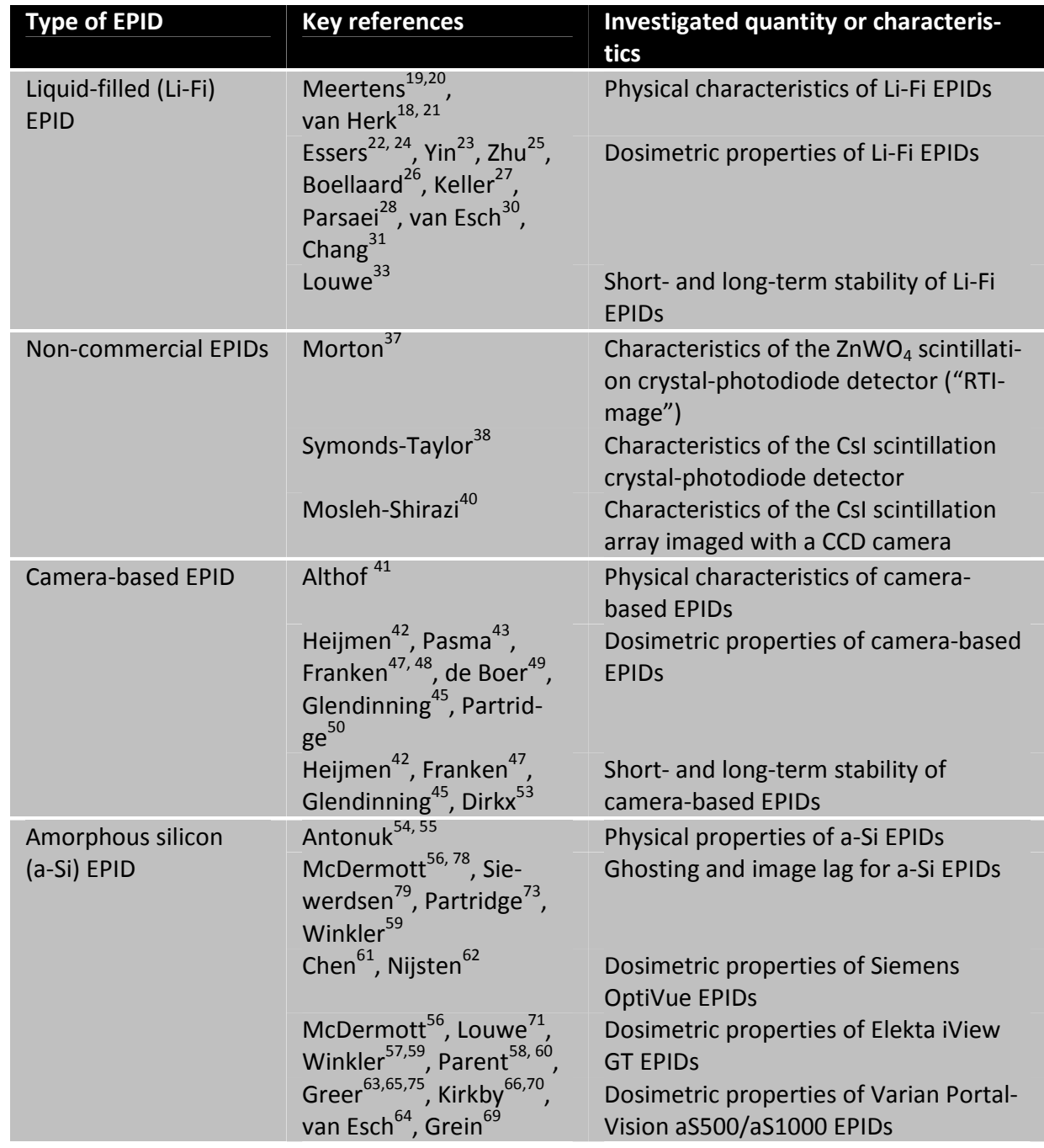




\section{EPID calibration: general approaches}

Two approaches have been adopted for the calibration of an EPID for dose measurements: conversion of the grey scale pixel value to a dose value or simulation (or prediction) of the grey scale pixel value.

The first approach applies (semi-) empirical models to convert the measured greyscale image of the EPID into a portal dose image, e.g. ${ }^{42,61,73,81,82}$. The EPID signal is converted to dose by using a calibrated detector, usually an ionisation chamber inside a water- or a mini-phantom, or film. ${ }^{35,76}$ The advantage of this approach is that the results can be verified independently with an ionisation chamber or film measurement. In other words, the interpretation of portal dose images is now simplified to the interpretation of the measurement of a conventional dosimeter. A possible drawback is that empirical or measurement-based models must be validated also outside the reference situation; especially the robustness of these calibration models under various different clinical situations needs to be tested.

The second approach simulates or models the detector response, usually by Monte Carlo (MC) simulation ${ }^{67,68,83}$ or by empirical models ${ }^{64}$. For accurate simulations, a detailed model of the EPID is necessary; however accurate technical details are not always available and often assumptions with respect to the construction and materials of the various layers are made. If the detector can be modelled accurately, an advantage of simulating the detector response is that the model can be directly verified by EPID measurements. A disadvantage in simulation methods is that for treatment verification, it is difficult to interpret patient dose differences directly from predicted and measured response differences. In principle it is possible to determine the fluence that resulted in the response (measured or predicted), and from this the corresponding dose to the patient, or detector, can be calculated. However different sources of error can produce similarly erroneous response measurements. The simulation approach is useful for quality control of the linac and treatment verification by means of a consistency check with the patient treatment plan.

The two approaches, either conversion or simulation method, have been used for different purposes. The conversion method usually applied a model-based approach that is simpler and faster than a Monte Carlo simulation of the EPID response, and therefore more suited for implementation in clinical routine. The drawback is that these calibration models can be too simple to cover all treatment techniques and irradiation configurations. Simulation of the detector response is a useful tool to derive, test and validate assumptions concerning the dose-response characteristics of the EPID. However, detailed information on the technical design of the EPID and long calculation times are needed. Accurate simulations could be used to develop models that convert the measured portal image to a portal dose distribution that opens various possibilities for (pre-) treatment dosimetry verification, as described in the later sections. 


\section{METHODS OF EPID DOSIMETRY}

There is no clear consensus in the literature on the definition of various procedures and methods related to EPID dosimetry. Here are definitions of various terms as used in this review, and further illustrated in Figure 2.2.

Verification procedures can be classified according to whether they are performed during treatment time (i.e. with the patient) or outside of treatment time (i.e. without the patient).

- Pre-treatment verification: a procedure comparing the whole or part of the intended treatment plan with measurements of corresponding radiation beams delivered by the linear accelerator outside patient treatment time, i.e. with open fields or a phantom. This comparison can focus on different aspects of the planned treatment: e.g. predicted and measured leaf positions, dose delivered to the detector or phantom, or incident energy fluence extracted from measurements.

- Treatment verification: a comparison of all or part of the planned and the delivered dose distribution based on measurements acquired during radiotherapy of the patient. These measurements can be used to determine the dose delivered to the detector or patient, or incident energy fluence obtained from measurements.

Dosimetry methods, independent of the type of detector used, can be grouped according to whether or not beams have passed through an attenuating medium, or whether the dose is reconstructed inside a phantom or patient.

- Non-transmission (or non-transit) dosimetry: determination of the dose in the detector, patient or phantom, or determination of the incident energy fluence, based on measurements without an attenuating medium between the source and the detector, i.e. phantom or patient.

(a)

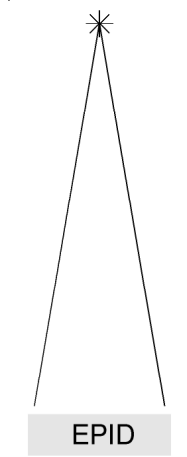

non-transmission pre-treatment (b)

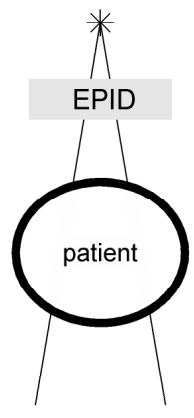

non-transmission during treatment (c)

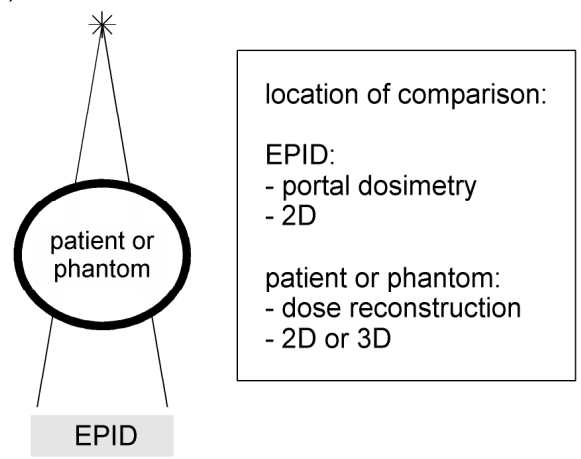

transmission pre- \& during treatment

Figure 2.2: Three arrangements for EPID dosimetry, each with the possibility to verify a dose distribution at the EPID level or inside the patient or phantom. 
- Transmission (or transit) dosimetry: determination of the dose at the position of the detector, patient or phantom, or determination of the incident energy fluence, based on radiation transmitted through the patient or phantom.

- In-phantom dosimetry: measurement or determination of the dose inside a phantom (rarely performed with EPIDs but included for completeness). This can be the dose at points, lines, planes or volumes within the phantom.

- In vivo dosimetry: measurement or determination of the dose inside the patient. Measurements performed during treatment can be performed invasively, i.e. inside the patient, or non-invasively, i.e. on or at some distance from the patient, whereby the in vivo dose at the point of interest is obtained by extrapolation. EPID dosimetry and the use of dosimeters positioned on the skin of a patient belong to the second category of in vivo dosimetry.

The dose can be measured and determined at different locations with different configurations of the EPID, phantom or patient, as described in Figure 2.2. Abbreviations: $E$ : level of the EPID, P: at the patient level / inside the patient.

a) non-transmission pre-treatment dosimetry: acquire image for each field without a patient or phantom in the beam.

- E: compare image (raw or converted to dose-to-water) with predicted EPID response or dose-to-water at the level of the imager, also referred to as 'portal dosimetry'.

- P: compare dose reconstructed inside patient/phantom CT scan (convert image to energy fluence, use as input for dose calculation algorithm) with plan calculated with patient/phantom CT scan.

b) non-transmission treatment dosimetry: acquire image for each field with the detector located between source and patient (during treatment), usually attached to the linac-head.

- E: compare image (raw or converted to dose-to-water) with predicted EPID response or dose-to-water at the level of the imager during treatment time, also referred to as 'portal dosimetry'.

- P: compare dose reconstructed inside patient/phantom CT scan (convert treatment image to energy fluence, use as input for dose calculation algorithm) with plan calculated with patient/phantom CT scan.

c) transmission treatment dosimetry: acquire image for each field with the detector located behind patient or phantom.

- E: compare image (raw or converted to dose-to-water) with predicted EPID response or dose-to-water at the level of the imager, behind the patient/phantom, also referred to as 'portal dosimetry'.

- P: compare reconstructed dose inside patient CT scan either back-project primary signal (simple correction-based algorithm) or convert image to energy fluence, use as input for a dose calculation algorithm with plan calculated with patient CT scan. 


\section{NON-TRANSMISSION EPID DOSIMETRY}

When considering EPID dosimetry, it is important to understand the capabilities and limitations of various possible methods to determine precisely what can be verified. In this chapter we describe non-transmission dosimetry methods, including 2D verification of the delivered energy fluence or dose distribution at the level of the EPID, and $3 \mathrm{D}$ verification, where the dose is reconstructed in a phantom or patient $\mathrm{CT}$ scan based on $2 \mathrm{D}$ images. For non-transmission dosimetry, both $2 \mathrm{D}$ and $3 \mathrm{D}$ verification methods are based on EPID measurements acquired without a phantom or patient between the radiation source and the detector. See Table 2.2 for an overview of methods and key references presented in the literature.

Non-transmission images can be useful for performing quality control of treatment parameters independent of the patient, related to dosimetric and geometric characteristics of the linear accelerator. Test fields have been designed to check the field flatness or symmetry of the beam, absolute output of the linear accelerator and radiation-light field coincidence. ${ }^{52,53,84-86}$ Furthermore, a large number of papers has been published on the use of EPIDs for the geometrical verification of MLC leaf positions or leaf trajectories during dynamic multileaf collimation. ${ }^{52,53,84,86-97}$ Most of these applications are based on using a gray-scale image not converted into a portal dose image, although generally these methods can also be used with portal dose images. These methods report in general an accuracy of about $1 \mathrm{~mm}$.

Furthermore, Vieira et al. ${ }^{98}$ proposed a simple leaf trajectory check for dynamic treatments. They used a sliding field with a constant gap and measurements with a camera-based EPID were performed. Differences in gap width as small as $0.2 \mathrm{~mm}$ could be detected making it an accurate QA procedure. The same group also described a QA procedure for the verification of MLC calibration and the delivery of static segmented MLC fields combined with a verification procedure for the absolute output verification of low dose segments. ${ }^{86}$ Differences smaller than 1\% (1SD) in output compared to ionization chamber measurements were obtained. Partridge et al. ${ }^{99}$ compared different methods for the verification of dynamic MLC treatments based on various EPID types: an in-house developed imager ${ }^{40}$, a method based on the liquid-filled EPID ${ }^{100}$ that was later extended for a-Si EPIDs ${ }^{101}$, and a method for a camera-based EPID ${ }^{102}$. With all methods it was possible to verify the position of the leaves during dynamic delivery with an accuracy of approximately $1.0 \mathrm{~mm}$.

\section{D verification based on non-transmission images}

Verification of the energy fluence or dose by means of an EPID is a common approach to ensure accurate delivery of radiotherapy beams. Two strategies have been adopted for verification of the planned beams: a comparison performed at the level of the detector or a method that reconstructs the dose distribution inside the patient or phantom based on non-transmission portal images. The fluence or the portal dose is either exported from the TPS, or calculated independently of the TPS from planned treatment parameters. The energy fluence is used to predict either the EPID response 
or the portal dose; i.e. the EPID signal is generally converted to a distribution of doseto-water deposited at the level of the EPID.

Kirkby et al. ${ }^{66}$ and Li et al. ${ }^{103}$ investigated the importance and the consequences of spectral response of a-Si EPIDs for dosimetric calibration showing that on one hand additional copper shielding could reduce the over-response of detectors to low-energy

Table 2.2: List of key references on non-transmission based dose verification methods.

\begin{tabular}{|c|c|c|c|}
\hline $\begin{array}{l}\text { Verification } \\
\text { procedure }\end{array}$ & $\begin{array}{l}\text { Type of } \\
\text { verification }\end{array}$ & Key references & $\begin{array}{l}\text { Objective of verification or subject of } \\
\text { the study }\end{array}$ \\
\hline \multirow{6}{*}{$\begin{array}{l}\text { QA of } \\
\text { treatment } \\
\text { machine }\end{array}$} & QA & Prisciandaro $^{93}$ & Radiation-light field congruence \\
\hline & QA & $\begin{array}{l}\text { Dirkx } \\
\text { Budgell }^{52,53}, 87\end{array}$ & $\begin{array}{l}\text { Linac output, beam profile flatness and } \\
\text { symmetry }\end{array}$ \\
\hline & QA & Baker $^{84}$, Yang ${ }^{95}$, & MLC leaf position for step-and-shoot \\
\hline & & $\begin{array}{l}\text { Samant } \\
\text { Parent }^{97}\end{array}$ & \\
\hline & QA & Vieira $^{86}$ & $\begin{array}{l}\text { MLC leaf position and absolute output for } \\
\text { low MU segmented fields }\end{array}$ \\
\hline & QA & $\begin{array}{l}\text { Vieira }{ }^{98}, \\
\text { Partridge }^{99}, \\
\text { Chang }^{88}\end{array}$ & $\begin{array}{l}\text { MLC leaf position during dynamic treat- } \\
\text { ment }\end{array}$ \\
\hline \multirow{5}{*}{$\begin{array}{l}\text { 2D fluence or } \\
\text { dose verifica- } \\
\text { tion at EPID } \\
\text { level }\end{array}$} & $\begin{array}{l}\text { pre- } \\
\text { treatment }\end{array}$ & Chang $^{82,106}$ & Dose profiles \\
\hline & & Siebers ${ }^{68}$ & Measured EPID response and Monte \\
\hline & treatment & Parent $^{58}$ & Carlo simulation \\
\hline & $\begin{array}{l}\text { pre- } \\
\text { treatment }\end{array}$ & Van $\operatorname{Esch}^{30,64}$ & $\begin{array}{l}\text { 2D energy fluence verification using Li-Fi } \\
\text { and a-Si EPIDs }\end{array}$ \\
\hline & $\begin{array}{l}\text { pre- } \\
\text { treatment }\end{array}$ & Nijsten $^{44}$ & $\begin{array}{l}\text { Absolute dose at center of field, field } \\
\text { shapes and wedge angles }\end{array}$ \\
\hline \multirow[t]{2}{*}{$\begin{array}{l}2 \mathrm{D} \text { dose re- } \\
\text { construction } \\
\text { in phantom }\end{array}$} & $\begin{array}{l}\text { pre- } \\
\text { treatment }\end{array}$ & Warkentin $^{109}$ & $\begin{array}{l}\text { Reconstruction of the dose in a plane in a } \\
\text { phantom based on non-transit portal } \\
\text { dose images }\end{array}$ \\
\hline & & $\begin{array}{l}\text { Cozzi }^{110} \\
\text { Moran }^{111} \\
\text { Nicolini }^{112}\end{array}$ & $\begin{array}{l}\text { Dose distribution measured with an EPID } \\
\text { positioned inside a phantom }\end{array}$ \\
\hline \multirow{6}{*}{$\begin{array}{l}\text { 3D dose re- } \\
\text { calculation in } \\
\text { phantom or } \\
\text { planning CT }\end{array}$} & \multirow{4}{*}{$\begin{array}{l}\text { pre- } \\
\text { treatment } \\
\text { pre- } \\
\text { treatment } \\
\text { pre- } \\
\text { treatment }\end{array}$} & van Elmpt ${ }^{116,117}$ & $\begin{array}{l}\text { Dose distribution reconstructed inside } \\
\text { (in)homogeneous phantoms }\end{array}$ \\
\hline & & Ansbacher ${ }^{115}$ & $\begin{array}{l}\text { Dose distribution reconstructed inside a } \\
\text { virtual phantom }\end{array}$ \\
\hline & & Van Zijtveld ${ }^{121}$ & Calculated dose distribution (TPS) using \\
\hline & & Steciw $^{120}$ & measured non-transit portal dose images \\
\hline & $\begin{array}{l}\text { pre- } \\
\text { treatment }\end{array}$ & van Elmpt & $\begin{array}{l}\text { Calculated dose distribution (Monte } \\
\text { Carlo) using the patient planning CT scan } \\
\text { based on measured non-transit portal } \\
\text { dose images }\end{array}$ \\
\hline & $\begin{array}{l}\text { pre- } \\
\text { treatment }\end{array}$ & Renner ${ }^{119}$ & $\begin{array}{l}\text { Calculated dose distribution (indepen- } \\
\text { dent dose engine) using the patient } \\
\text { planning CT scan based on measured } \\
\text { non-transit portal dose images }\end{array}$ \\
\hline
\end{tabular}


components, while on the other hand, when convolution/superposition methods are used to predict EPID images, the incorporation of a beam-hardening dependence of the EPID kernel would improve dosimetric accuracy. Chytyk et al. ${ }^{104}$ investigated the effect of tilted dose kernels for portal dose prediction and found that if one assumes parallel dose kernels, the dosimetric effect is less than $1.8 \%$ in extreme cases and less than $1.0 \%$ in most clinically relevant situations. Yeboah et al. ${ }^{105}$ used Monte Carlo simulations to model the EPID response and found that the air gap and field size are roughly complementary and suggested that an equivalent field size concept may be used to account for intensity and spectral changes arising from air gap variations.

Several other groups developed models to predict the EPID response to compare with non-transit EPID images of treatment fields. ${ }^{30,31,44,51,58,64,68,106-108}$ Parent et al. ${ }^{58}$ also used a Monte Carlo model, and predicted the image of a test IMRT field delivered on the beam axis and with an offset. A variation of EPID response up to $28 \%$ was found between the on- and off-axis delivery. Chang et al. ${ }^{31,106}$ compared measured and planned relative profiles and central axis dose of 25 prostate fields. After their correction methods were applied, very good agreement was obtained, with a difference of $1.9 \%( \pm 0.5 \%, 1 \mathrm{SD})$. The agreement was better than $2.0 \%$ for the central axis dose. Nijsten et al. ${ }^{44}$ reported a method to verify the absolute point dose and the $2 \mathrm{D}$ geometrical parameters of the treatment fields at the level of the EPID, to check the manual treatment parameter transfer from TPS to accelerator.

Besides predicting the dose at the imager level or the EPID response, another approach is to convert the energy fluence (measured without a phantom) to a 2D dose distribution inside a phantom at a specific depth. This method, first proposed by Warkentin et al. ${ }^{109}$, involves deconvolution of the raw EPID images to two-dimensional distributions of primary fluence using Monte Carlo generated scatter kernels. The phantom dose-distribution that would result from the energy fluence derived from measurements is then reconstructed. The calculated dose distribution inside this phantom at a specific depth, e.g. $10 \mathrm{~cm}$, can then be compared to treatment planning dose plane at that depth. In this way a direct comparison between the dose distribution calculated by the TPS and the delivered in-phantom dose distribution can be made.

A method that is more difficult to classify involves pre-treatment verification by inserting the EPID inside a phantom, or between attenuating material, at the position of the patient. ${ }^{110-112}$ The material above the detector effectively acts as build-up layer, without an air-gap, so therefore it can also be considered as a non-transmission EPID dosimetry approach. Agreement with film has been obtained within $4 \%, 3 \mathrm{~mm}$ for phantom depths of smaller than $4 \mathrm{~cm}^{112}$

Another possible arrangement for EPID dosimetry is 'non-transit imaging during treatment', by mounting the EPID to linac head (Figure 2.2b). Commercial development of this strategy using an EPID is however still at an early stage. ${ }^{113}$ There are various other dosimetry devices such as ionisation chamber matrices (i.e. they are not EPIDs) with the same configuration. ${ }^{114}$ The idea is to image beams when delivered to the patient through the detector. The signal acquired during treatment is converted to the 'delivered energy fluence', and may be compared with the 'planned energy fluence'. The advantage of using EPIDs is that they have a much higher resolution than the current matrix detectors. 


\section{D dose reconstruction based on non-transmission images}

Another approach to verification based on non-transmission dosimetry is the reconstruction of the 3D dose distribution based on measured portal (dose) images. The idea is that with both the 2D energy fluence delivered by the linear accelerator (derived from portal images of each beam) and a dose calculation algorithm, one can reconstruct a dose distribution in 3D based on measurements. Images can be acquired pre-treatment (Figure 2.2a) or during treatment (Figure 2.2b). The reference dose distribution can be calculated with a CT scan of either a phantom or the patient, as appropriate.

For 3D in-phantom dose reconstruction, a method was described by Ansbacher. ${ }^{115}$ The delivered segments of an IMRT treatment were measured and reconstructed in a pre-defined cylindrical 'virtual phantom' of fixed diameter using a set of attenuation and phantom scatter correction factors. Absolute point dose values and relative dose profiles were both reconstructed with an error smaller than $2 \%$ (1 SD), compared to ionisation chamber and film measurements for a group of 20 head-and-neck cancer IMRT patients analysed retrospectively.

An alternative algorithm was described by van Elmpt et al. ${ }^{116-118}$, who used the energy fluence extracted from a measured portal dose image as input for a Monte Carlo dose calculation in an object of choice. Reconstruction was based on either a CT scan of a phantom or the planning CT scan of a patient. For both homogeneous and inhomogeneous phantoms, agreement within 3\% of the dose measured using film or ionisation chamber dosimetry was achieved. In principle any dose calculation engine could be used, however a Monte Carlo-based approach provides greater accuracy, especially for inhomogeneous geometries. Renner et al. ${ }^{119}$ extracted the energy fluence from EPID measurements and applied an in-house developed dose calculation algorithm using the planning CT scan. A similar approach was used by Steciw et al. ${ }^{120}$, but these authors applied the same TPS as used for the original dose calculation. The advantage of these methods is that the transfer and delivery of the plan, the creation of the initial energy fluence file and the modelling of the MLC by the TPS are all checked. However certain aspects of the dose calculation, occurring after the creation of the fluence distribution, remain unchecked if the same TPS is used to calculate both the planned and reconstructed dose distributions. Van Zijtveld et al. ${ }^{121,122}$ used a method developed previously by McNutt et al. ${ }^{123,124}$ and applied it to non-transmission measurements. In this method, the incident primary energy fluence of the plan (exiting the treatment head) is adjusted until it converges to the measured portal image. The fluence is then used to re-calculate the $3 \mathrm{D}$ dose distribution using a cone-beam CT scan acquired just prior to treatment.

The main advantage of 3D verification methods using planning CT scans is that differences between planned and delivered beams may be assessed in terms of dose in the target volume or dose in normal tissue, resulting in a pre-treatment verification method that is more intuitive compared to conventional methods based on measurements at points or in planes. Errors in the dose calculation of the TPS can be detected along with the plan deliverability, such as correct data transfer and mechanical or dosimetric functioning of the linear accelerator. Furthermore, with pre-treatment verification all errors due to patient anatomy changes, patient set-up or treatment 
machine malfunctioning that occur during treatment will be missed. Dose reconstruction methods that do not include patient information at the time of treatment are potentially misleading, so users should be aware of their limitations (for more detail, see the section Clinical approaches to EPID dosimetry).

\section{TRANSMISSION EPID DOSIMETRY}

The ultimate aim of radiotherapy verification is to verify that the patient is receiving the correct dose during treatment. To this end, it is becoming increasingly advantageous to determine the dose from transmission images, whereby the beam passes through the patient, as described in Figure 2.2c. In this way the dose can be verified either at the level of the EPID or by reconstructing the dose inside a digital representation of the patient. While dosimetry with or without a phantom (pre-treatment transit or non-transit dosimetry) provide similar information, the reason for doing transit dosimetry behind a phantom is to be able to extend such a procedure to patient treatment verification. Models based on homogeneous phantoms need to be adapted to account for inhomogeneities inside the patient. For verification during treatment, the closer this representation resembles the patient at the time of treatment (e.g. by using a set of (cone-beam) CT scans), the more accurate this validation will be. EPID dosimetry based on transmission images is described in this section, and classified by point-dose verification, 2D portal dose prediction, 2D back-projection models and finally $3 D$ dose reconstruction models. Various methods and references for transmission dosimetry are summarised in Table 2.3.

\section{Point-dose verification based on transmission images}

The motivation for point dose verification based on transmission EPID images was to replace diode in vivo dosimetry. ${ }^{125}$ In vivo dosimetry at a point has been useful for detecting large errors in the delivery of conformal fields re-occurring at successive treatment fractions. Usually in vivo dosimetry is performed using diodes positioned at the entrance or exit side (or both) of the patient. The time required to position the diodes on the patient, to maintain an accurate calibration and to develop a 'trustworthy' system is rather laborious and therefore a disadvantage. Furthermore the shadowing effect of the entrance diode, having a relatively thick build-up cap, may compromise its use. Point dose verification based on EPID measurements, therefore, has been an approach initiated by various groups from the onset of EPID dosimetry. Point dose measurements made behind the patient have been compared at the position of the EPID ${ }^{126}$, extrapolated (back-projected) to a point within the patient (or phantom), at $5 \mathrm{~cm}$ depth $^{127,128}$ and at the isocentre ${ }^{82,129-131}$.

Pasma et al. ${ }^{127}$ and Nijsten et al. ${ }^{128}$ correlated the dose measured with the EPID on the central beam axis with dose values at $5 \mathrm{~cm}$ depth using a (semi-) empirical relationship between these two quantities. Chang et al. ${ }^{82}$ and Piermattei et al. ${ }^{130}$ described similar methods to reconstruct the absolute dose at the isocentre of a phantom, by calibrating the EPID under the same scatter conditions as the actual measurements, 
i.e. using a set of correlation factors between the EPID signal and the dose at the isocentre.

\section{D verification based on transmission images (verification at the EPID level)}

A popular approach to transmission dosimetry involves predicting the portal dose at the level of the imager behind a phantom or patient. ${ }^{22,43,49,132-141}$

At the Erasmus Medical Center - Daniel den Hoed Cancer Center in Rotterdam (The Netherlands), the portal image was predicted behind patient CT scans. Pasma et al. ${ }^{43,132}$ began by comparing corrected EPID images against ionisation chamber measurements, including modulated fields, obtaining agreement up to $1 \%$. De Boer et al. ${ }^{49}$ compared EPID portal dose images and ionisation chamber measurements (at the detector plane) behind an absorber for various field shapes. The average difference in absolute dose was $0.1 \pm 0.5 \%$ ( $1 \mathrm{SD}$ ) over the entire irradiation field, with no deviation

Table 2.3: List of key references on non-transmission based dose verification methods.

\begin{tabular}{|c|c|c|c|}
\hline $\begin{array}{l}\text { Verification } \\
\text { procedure }\end{array}$ & $\begin{array}{l}\text { Position of } \\
\text { verification: } \\
\text { EPID or patient } \\
\text { / phantom }\end{array}$ & $\begin{array}{l}\text { Key referen- } \\
\text { ces }\end{array}$ & $\begin{array}{l}\text { Objective of verification or subject of } \\
\text { the study }\end{array}$ \\
\hline \multirow[t]{3}{*}{$\begin{array}{l}\text { point dose } \\
\text { verification }\end{array}$} & Patient & Pasma $^{127}$ & $\begin{array}{l}\text { Measured point dose distribution back- } \\
\text { projected to the dose in a patient at } 5 \mathrm{~cm} \\
\text { depth }\end{array}$ \\
\hline & $\begin{array}{l}\text { EPID and } \\
\text { patient (in vivo) }\end{array}$ & Nijsten $^{128}$ & $\begin{array}{l}\text { Measured point dose verified at EPID } \\
\text { level and back-projected to the dose in a } \\
\text { patient at } 5 \mathrm{~cm} \text { depth }\end{array}$ \\
\hline & $\begin{array}{l}\text { Patient / } \\
\text { phantom (pre- } \\
\text { treatment and } \\
\text { in-vivo) }\end{array}$ & $\begin{array}{l}\text { Chang }^{82}, \\
\text { Piermattei } \\
131\end{array}$ & $\begin{array}{l}\text { Measured point dose back-projected to } \\
\text { the dose in a patient at the isocenter }\end{array}$ \\
\hline \multirow{7}{*}{$\begin{array}{l}2 \mathrm{D} \text { transit } \\
\text { dose verifica- } \\
\text { tion at EPID } \\
\text { level }\end{array}$} & EPID & $\begin{array}{l}\text { Pasma } \\
\text { van Elmpt }^{132,141}\end{array}$ & $\begin{array}{l}\text { Predicted transit portal dose distribution } \\
\text { using measured beam data and the } \\
\text { planning CT scan }\end{array}$ \\
\hline & EPID & Kroonwijk $^{142}$ & Clinical results for prostate \\
\hline & EPID & $\begin{array}{l}\text { Dahlgren } \\
140\end{array}$ & $\begin{array}{l}\text { Predicted transit portal dose using the } \\
\text { collapsed cone superposition method }\end{array}$ \\
\hline & EPID & Spezi ${ }^{45}$ & Portal dose image prediction using \\
\hline & $\begin{array}{l}\text { extraction of } \\
\text { entrance } \\
\text { fluence }\end{array}$ & $\begin{array}{l}\text { Hansen }^{150} \\
\text { Fielding }^{144^{\prime}} \\
\text { Vieira }^{143} \\
\text { Spies }^{148}\end{array}$ & Extract entrance energy fluence \\
\hline & EPID & McCurdy $_{136-138}^{67}$ & $\begin{array}{l}\text { Predicted portal dose using Monte Carlo } \\
\text { based scatter kernels }\end{array}$ \\
\hline & EPID & $\begin{array}{l}\text { McNutt }^{152}, \\
\text { Reich }^{34}, \\
\text { Mohammadi } \\
35,154\end{array}$ & $\begin{array}{l}\text { Predicted portal dose using a TPS by } \\
\text { adding the EPID to the planning CT scan } \\
\text { ("extended phantom") }\end{array}$ \\
\hline
\end{tabular}


Table 2.3 (continued).

\begin{tabular}{|c|c|c|c|}
\hline $\begin{array}{l}\text { Verification } \\
\text { procedure }\end{array}$ & $\begin{array}{l}\text { Position of } \\
\text { verification: } \\
\text { EPID or patient } \\
\text { / phantom }\end{array}$ & $\begin{array}{l}\text { Key referen- } \\
\text { ces }\end{array}$ & $\begin{array}{l}\text { Objective of verification or subject of } \\
\text { the study }\end{array}$ \\
\hline \multirow[t]{5}{*}{$\begin{array}{l}\text { 2D transit } \\
\text { dose verifica- } \\
\text { tion at } \\
\text { patient level }\end{array}$} & $\begin{array}{l}\text { Patient / } \\
\text { phantom (in } \\
\text { vivo) }\end{array}$ & $\begin{array}{l}\text { Essers }^{22,126} \\
\text { Kirby }^{156,157^{\prime}} \\
\text { Boellaard }^{81} \\
158\end{array}$ & Reconstructed exit dose (3D CRT) \\
\hline & $\begin{array}{l}\text { Patient / } \\
\text { phantom (in } \\
\text { vivo) }\end{array}$ & $\begin{array}{l}\text { Boellaard } \\
160\end{array}$ & $\begin{array}{l}\text { Reconstructed 2D midplane dose (3D } \\
\text { CRT) }\end{array}$ \\
\hline & $\begin{array}{l}\text { Phantom (pre- } \\
\text { treatment) }\end{array}$ & Wendling $^{76}$ & Reconstructed 2D midplane dose (IMRT) \\
\hline & $\begin{array}{l}\text { Patient / } \\
\text { phantom (pre- } \\
\text { treatment } \\
\text { and in-vivo) }\end{array}$ & $\begin{array}{l}\text { McDer- } \\
\text { mott }^{161,162}\end{array}$ & Clinical results for prostate IMRT \\
\hline & $\begin{array}{l}\text { Phantom (pre- } \\
\text { treatment) }\end{array}$ & Talamonti $^{77}$ & $2 \mathrm{D}$ verification (IMRT) \\
\hline \multirow{6}{*}{$\begin{array}{l}\text { 3D dose } \\
\text { verification } \\
\text { (dose } \\
\text { calculated } \\
\text { using a CT } \\
\text { scan ac- } \\
\text { quired from } \\
\text { the planning } \\
\text { stage or } \\
\text { acquired 'in } \\
\text { room') }\end{array}$} & $\begin{array}{l}\text { Patient (plan- } \\
\text { ning CT) }\end{array}$ & Hansen $^{39}$ & $\begin{array}{l}\text { Back-projected dose based on transmis- } \\
\text { sion EPID images and planning CT scan }\end{array}$ \\
\hline & $\begin{array}{l}\text { Patient (plan- } \\
\text { ning } \mathrm{CT} \text { ) }\end{array}$ & Jarry $^{163}$ & $\begin{array}{l}\text { Back-projected energy fluence based on } \\
\text { EPID images and a Monte Carlo calcula- } \\
\text { tion using the planning CT scan }\end{array}$ \\
\hline & $\begin{array}{l}\text { Patient (plan- } \\
\text { ning CT) }\end{array}$ & McNutt $^{123,124}$ & $\begin{array}{l}\text { Combined EPID transit dose measure- } \\
\text { ment and planning CT scan in an "ex- } \\
\text { tended phantom" using the TPS }\end{array}$ \\
\hline & $\begin{array}{l}\text { Patient (plan- } \\
\text { ning } C T \text { ) }\end{array}$ & Louwe $^{166}$ & $\begin{array}{l}\text { Reconstructed 3D dose distribution } \\
\text { (breast) }\end{array}$ \\
\hline & $\begin{array}{l}\text { Patient (in } \\
\text { room CT) }\end{array}$ & $\begin{array}{l}\text { McDer- } \\
\text { mott }^{167}\end{array}$ & $\begin{array}{l}\text { Reconstructed in vivo dose for rectal } \\
\text { cancer patients using cone-beam CT scan }\end{array}$ \\
\hline & $\begin{array}{l}\text { Patient (in } \\
\text { room CT) }\end{array}$ & Partridge $^{164}$ & $\begin{array}{l}\text { Back-projected dose based on transmis- } \\
\text { sion EPID images and MV cone-beam CT }\end{array}$ \\
\hline
\end{tabular}

larger than $2 \%$. This strategy has also been used during treatment to investigate organ motion. ${ }^{142}$ The various research and clinical approaches used by this group have been summarised in a report by Dirkx et al. ${ }^{134}$ These authors and other groups have also proposed methods to separate the modulated beam component from the anatomical information to check the entrance fluence independently. ${ }^{143,144}$

Spezi and Lewis ${ }^{145}$ developed a planar calibration of the Li-Fi EPID response using a full Monte Carlo simulation of a radiotherapy treatment facility including an MLC and EPID. The calibrated EPID was used as a dosimetric system to validate a MC model for the MLC. Computations and measurements agreed within $2 \%$ of dose difference (or 2 $\mathrm{mm}$ in regions of high dose gradient). Spies et al. ${ }^{146,147}$ developed a scatter model for portal images using Monte Carlo generated data, followed by a method to reconstruct incident beam profiles from images acquired behind a phantom. ${ }^{148}$ Their method was 
based on an iterative algorithm that compensated for beam attenuation and subtracting phantom scatter reaching the detector, yielding an accuracy for the incident beam distribution of better than 3\% (relative difference). Researchers at the Royal Marsden Hospital, London (UK) presented Monte Carlo simulations and a simple physical model for scatter correction. ${ }^{149}$ Hansen et al. ${ }^{150}$ extracted the primary signal from transmission measurements, accurate to within 1.5\% (1 SD) for scatter-to-primary ratios up to $25 \%$. Later Partridge and Evans ${ }^{151}$ described the practical range for which the model was valid; for centre-of-mass to detector distances larger than $60 \mathrm{~cm}$ and field areas up to $625 \mathrm{~cm}^{2}$.

Several groups have used the TPS to calculate the transmitted dose map at the EPID level ${ }^{34,35,152-154}$, as well as relative fluence maps for tomotherapy systems ${ }^{155}$. Phantom thickness changes of $\sim 1 \mathrm{~mm}$ have been detected, corresponding to dose changes on the central beam axis smaller than $0.6 \%{ }^{34}$ In a later study these authors reported that both for homogeneous and inhomogeneous phantoms, more than $90 \%$ agreement was achieved using dose-difference and distance-to-agreement criteria of $2 \%, 3$ $\mathrm{mm}$ or $3 \%, 2.5 \mathrm{~mm}^{153}$

\section{D back-projection models based on transmission images (verification at the patient or phantom level)}

In order to verify the patient dose in vivo, it is necessary to make a measurement at the same time that the dose is being delivered to the patient and use that measurement to determine the dose inside the patient. To do this with EPID dosimetry, two things are required: transmission measurements and an algorithm to reconstruct the dose in the patient from the EPID signal.

One of the first back-projection methods described in the literature deals with exit dose reconstruction. The EPID has been used for this purpose by several groups. ${ }^{81,126,156-158}$ The main objective was to replace diode exit dosimetry during treatment, with an accuracy reported to be within $3 \%$ compared with diode measurements behind phantoms. ${ }^{156}$ Parsaei et al. ${ }^{28}$ used the Li-Fi EPID to measure relative exit dose profiles behind a phantom. Open and wedged dose profiles measured with the LiFi EPID agreed with ionisation chamber measured profiles to within 3.5\%.

Zhu et al. ${ }^{25}$ used a Li-Fi EPID to back-project pixel values to a 2D dose map of an ionisation chamber matrix measured at the depth of dose maximum $\left(D_{\max }\right)$ of $1.5 \mathrm{~cm}$ in water. Boellaard et al. ${ }^{159,160}$ subsequently introduced a back-projection midplane-dose dose reconstruction model for the Li-Fi EPID. The calibration of the EPID for backprojection dosimetry consisted of two parts. First, a dosimetric calibration established the dose-response relationship by relating EPID pixel values to dose values at the position of the imager. Secondly, the parameters for the back-projection algorithm, i.e. attenuation factors and scatter within the EPID and patient, and from patient to EPID, were determined to enable the conversion from the dose at the EPID position to the dose inside the patient or phantom. This model was extended to the a-Si EPID by Wendling et al. $^{76}$, who reported close agreement between EPID and film measurements using gamma evaluation with $2 \%, 2 \mathrm{~mm}$ dose-difference and distance-toagreement criteria. Clinical applications were reported by McDermott et al. ${ }^{161,162}$, 
where back-projected 2D EPID dosimetry replaced verification with film, and subsequently in vivo verification replaced pre-treatment checks for prostate treatments.

Spies et al. ${ }^{148}$ also investigated reconstructing the $2 \mathrm{D}$ incidence fluence based on a thickness map of the phantom, or transmission measurements. Pre-calculated Monte Carlo scatter kernels were used to estimate scatter. The method was tested for a homogeneous water-equivalent slab phantom for simple rectangular and complex multileaf collimated fields. They reported an accuracy for the incident beam distribution of better than $3 \%$ (relative difference).

An alternative calibration method was described by Talamonti et al. ${ }^{77}$ for pretreatment verification in a phantom. They used an EPID to measure exit doses and related pixel values to film dose distributions measured in a phantom at the patient level. Measurements were made for various phantom thickness, air gap distances, field sizes and measurement position in the phantom. These authors wanted to obtain a universal calibration factor, regardless of field size. To this end, a copper plate was added to attenuate low-energy phantom scattered photons which largely dependent on the field size of the irradiated volume. This method was used for the measurement of the dose distributions for 15 clinical IMRT fields. On average, $97.6 \%$ and $98.3 \%$ of the points agreed (using gamma criteria of $3 \%, 3 \mathrm{~mm}$ ) for EPID versus TPS and for EPID versus film, respectively.

\section{D dose reconstruction based on transmission images}

Three-dimensional dose reconstruction begins with a portal image taken behind a patient or phantom. Based on the information in this image, in combination with a 3D dose reconstruction model, the dose distribution for this segment or field can be reconstructed in 3D within the patient or a phantom. Finally, the 3D dose distributions of all fields may be summed to yield the total 3D dose distribution. In general, 3D dose reconstruction based on transmission dosimetry requires (a) modelling of the EPID response, including a correction for the scatter from the patient or phantom to the measured EPID image, (b) a 3D "density representation" of the patient or phantom, preferably including position and anatomy of various types of tissue and organs, and (c) a dose calculation algorithm.

Hansen et al. ${ }^{39}$ demonstrated this concept on a humanoid phantom. First, the EPID image was corrected for scatter from the phantom to the detector yielding the transmitted primary fluence. This fluence was then back-projected through the planning CT data set yielding the primary fluence within the phantom. By convolving this distribution with energy deposition kernels (taken from the literature) the dose in the phantom was calculated. The reconstructed dose distribution agreed with the dose distribution from the TPS within $2 \%$ in the pelvic region of a humanoid phantom. Jarry and Verhaegen $^{163}$ investigated the use of Monte Carlo calculations for this step and reached agreement within 5\% for IMRT treatments. The planning CT scan was used for this method and is only reliable for anatomy that is not changing during treatment.

Partridge et al. ${ }^{164}$ extended the model of Hansen et al. by incorporating an on-line imaging modality and performed a proof-of-principle experiment on an anthropomorphic phantom. The portal image was converted to primary fluence using an iterative phantom-scatter estimation. Next, the energy fluence was back-projected through a 
"treatment-time" MV (cone-beam) CT model of the phantom. For dose reconstruction, the same convolution/superposition algorithm as for the original planning dose distribution was used. Recently, Chen et al. ${ }^{165}$ also demonstrated the use of a MV conebeam CT data set, acquired just prior to treatment, for dose reconstruction within a patient. In their approach, no correction for the scatter from the patient to the EPID was included; hence reconstructed dose values were $10 \%$ higher than planned values.

Another concept was developed by the group from the Netherlands Cancer Institute - Antoni van Leeuwenhoek Hospital (NKI-AVL) in Amsterdam (The Netherlands) based on their back-projection algorithm for 2D dose reconstruction. The model was extended to multiple parallel planes perpendicular to each beam direction. In this way it was possible to get a full 3D dose reconstruction as demonstrated for breast cancer treatments using EPID images in combination with planning CT data. This information was used to determine the dose distribution in irradiated anthropomorphic breast phantoms. ${ }^{166}$ The scattered components of the dose within the EPID, from the phantom to the EPID, and within the phantom were taken into account by using empirical kernels. For the attenuation correction the external contours of the phantom were used, which were determined from the (planning) CT scan. The average deviation of the $3 \mathrm{D}$ reconstructed dose from the planned dose was $1.4 \%$ with a SD of $5.4 \%$. A later study combined the use of an a-Si EPID-based 3D in vivo dosimetry and cone-beam CT (CBCT) to obtain a complete account of the entire treatment for a selected patient group. For that purpose nine hypo-fractionated rectum cancer IMRT patient plans were investigated. ${ }^{167}$ Planned and measured in vivo 3D dose distributions showed excellent agreement, based on 3D gamma evaluation and dose-volume histograms (DVHs). Over-dosage of up to $4.5 \%$ was measured only in one fraction due to the presence of gas pockets.

McNutt et al. ${ }^{123,124}$ included the EPID with the CT data set into a so-called "extended phantom". With an iterative convolution/superposition algorithm the primary energy fluence at the EPID was adjusted until it converged to a fluence that would have produced the measured portal image. The converged primary energy fluence was back-projected and convolved with dose-deposition kernels. The dose distribution agreed within $3 \%$ with the planned dose distribution.

For the tomotherapy treatment machine, a procedure was developed by Kapatoes et al. ${ }^{168,169}$ A reconstruction of the incident energy fluence was made using the measured transit signal in the detector and a measured transfer function. This function was calculated from a database of calibration data combined with the on-line MV CT scan for the extraction of the radiological path lengths and patient-detector distances. The reconstructed energy fluence was then used as input for a dose calculation inside the MV CT scan. A phantom study ${ }^{169}$ showed that positional differences down to $2 \mathrm{~mm}$ or $2^{\circ}$ could be detected, but had negligible impact on the 3D dose distribution. Accelerator output differences or stuck leaves of the MLC could be detected and their influence on the 3D dose distribution be estimated. 


\section{CLINICAL APPROACHES TO EPID DOSIMETRY}

In the past, treatments were fairly standardised, so experienced radiographers, physicists and radiation oncologists knew what to expect in terms of sizes and shapes of treatment fields, and number of monitor units delivered, for a given type of treatment. With the introduction of advanced radiotherapy techniques and higher dose prescriptions, a wide range of parameters is possible, so deviations are less obvious and it is much more difficult to know when something is wrong. With the introduction of new data managements systems, change of TPSs and protocols, the risk of error also increases, even for a simple treatment technique. It is therefore even more surprising that the commercial development of EPID dosimetry is lagging far behind other advances such as patient positioning, i.e. image-guided radiotherapy (IGRT). To the best of our knowledge, there are only two commercial products available. The first one is produced by Varian, who released a dosimetric module in 'PortalVision' based on a publication by van Esch et al. ${ }^{64}$ Their method allows verification of pre-treatment, nontransmission fields, at the level of the EPID (Figure 2.2a). The second commercial product currently available is based on the method described by Renner et al. ${ }^{119}$ It uses a pre-treatment 3D dose verification method as described in the section on 3D nontransmission dosimetry. Despite the lack of commercial availability, several clinics have introduced in-house solutions and produced data describing the possibilities of EPID dosimetry in clinical practice. Before discussing the clinical experience using EPID dosimetry, an overview is presented of the type of errors that can be detected.

\section{Error detection potential using EPID dosimetry}

Descriptions of the various ways an EPID may be used to verify dose distributions in 2D/3D are summarised in Table 2.4. The methods are divided into two categories: pretreatment verification (image measured outside of treatment time, behind or without a phantom) and treatment verification (image measured before or behind the patient, dose determined inside the patient, in vivo, or at the detector level). Under 'potential errors', various possible sources of error in radiotherapy are listed and the type of EPID verification that is able to detect this error is also indicated.

Several types of errors can be distinguished. Machine errors are related to hardware faults, either random or systematic (occurring at every treatment fraction). Pretreatment measurements using EPIDs reveal systematic errors and may be used to estimate the probability of random machine errors that might occur during treatment. However, they cannot be used to detect random errors occurring during actual patient treatment, such as a change in linac output. If such a random error occurs during pretreatment verification, it can either be corrected or the measurement can be repeated yielding information about its average value and standard deviation. The detection of a wrong gantry angle, due to a mechanical failure, may be difficult with EPID dosimetry. Whether or not EPID dosimetry is sensitive to gantry angle errors is highly dependent on the treatment technique. A case where it might be possible to detect a wrong gantry angle is when transit measurements are made behind a non-homogeneous phantom or patient. 
Table 2.4 Overview of the various errors that can be detected with EPID dosimetry.

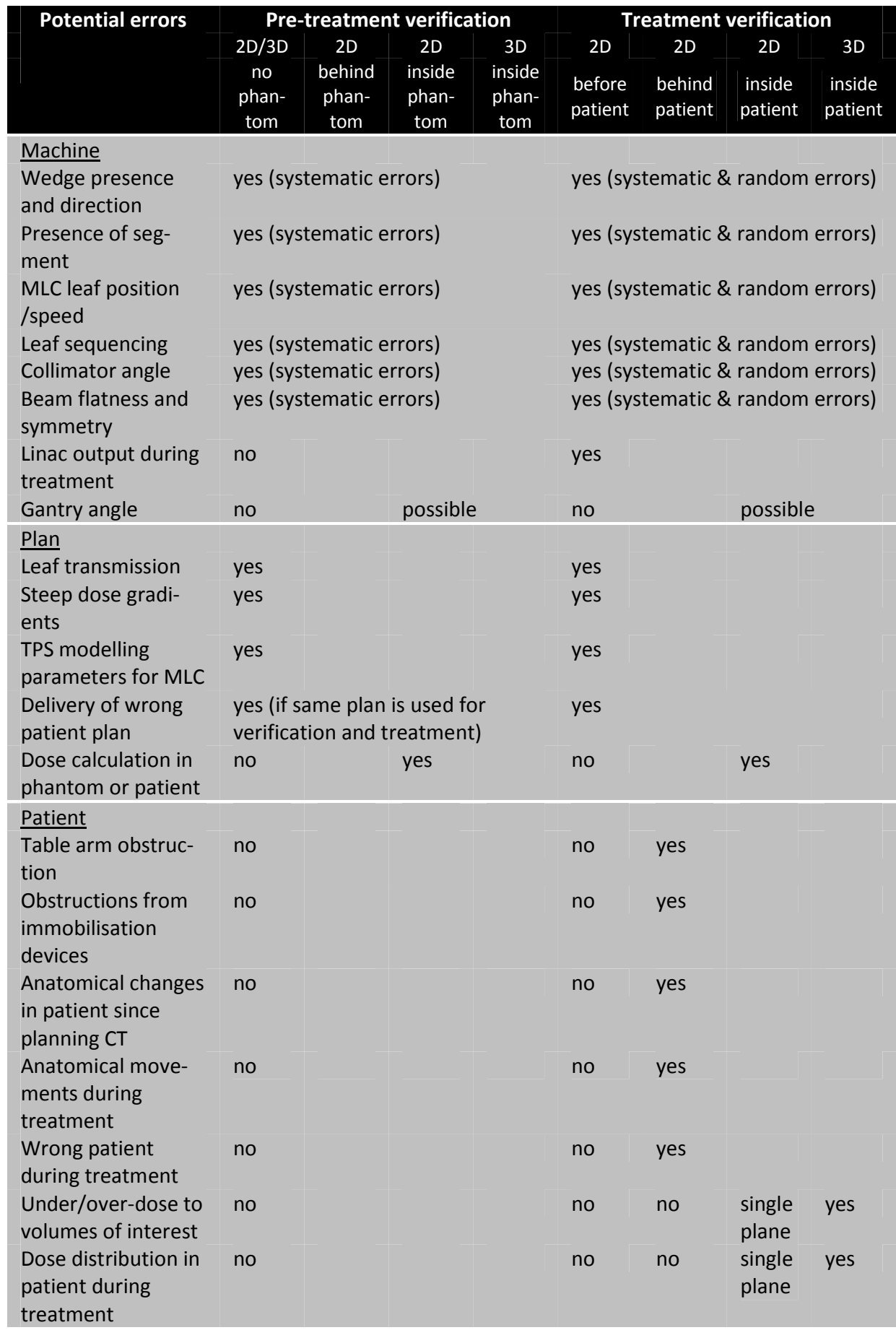


Wrong gantry angles may then result in different transmission images and therefore in a wrong dose distribution at the patient level. A problem arises when the transmission images do not change much and the unplanned angle results in the 'correct dose' delivered to the wrong location. Such a situation emphasises the need for dose reconstruction in combination with patient anatomy and set-up verification at the time of treatment, for instance by including an analysis of anatomical structures visible in EPID images. For transit or non-transit dosimetry in 3D, the contribution of dose from a wrong gantry is likely to render a different total dose distribution in certain cases.

Plan errors include dose calculation errors, wrong TPS commissioning data (such as beam fit errors) and data transfer errors, which includes the selection of a wrong patient plan. If the error is related to the heterogeneity of the patient anatomy, the error will not be detected by re-calculating the dose in a homogeneous phantom. EPIDbased (3D) dose reconstruction algorithms with an independent dose calculation that take into account inhomogeneities (e.g. Monte Carlo, convolution/superposition or collapsed-cone algorithms) are necessary for this type of verification. Errors due to poor modelling of the MLC, however, are likely to be detected by using 2D EPID verification models. As with gantry angle errors, selection of the wrong plan will most likely be detected by EPID transit dosimetry, but only if the transmission results in a different dose distribution. The error will not be detected if the measured and planned dose distributions are similar, even though the medium (i.e. the patient) is different.

Patient errors are specifically related to errors due to changes in the patient's positioning or anatomy from the situation at planning. Verification of the dose based on EPID measurements prior to treatment cannot detect any of these types of errors. Furthermore, at least one vendor is developing a system whereby EPID images are acquired with the detector between the beam source and the patient (Figure 2.2b). The detection of patient-related errors will be limited to situations where additional imaging information is available (usually acquired before and/or after treatment) and the patient did not change between imaging and treatment time. 2D EPID verification models during treatment are used to provide a consistency check for the dose delivered for treatment sites where no large differences in anatomy are expected. These methods are either based on the portal dose image or use a back-projection algorithm to reconstruct a dose-plane inside the patient. For treatment sites where anatomy changes are common it is difficult to say anything meaningful about the dose delivered to the region-of-interest (i.e. target volumes or organs at risk) or the total dose distribution. This will only be possible if the dose is determined in 3D and based on accurate 3D imaging data (e.g. cone-beam CT) acquired close to the time of treatment.

For machine, plan or patient related errors, pre-treatment and treatment EPID dosimetry raises an alarm before or from the first fraction (after other procedures and checks fail), alerting clinical staff to what often turns out to be a simple error with drastic consequences. Ideally, when EPID treatment images can be acquired for every fraction and analysed automatically, a check and record of the entire treatment will be guaranteed without any cost to patient treatment time or clinical workflow. 


\section{Acceptance and rejection criteria}

Quick and adequate interpretation of a verification result can be hampered by the fact that 2D and 3D images render vast amounts of information for the user. For dose distributions with high dose gradients, simple dose difference maps are usually insufficient to deduce clinically relevant differences between planned and measured dose distributions. Dose-difference and distance-to-agreement (DTA) analyses are extremely sensitive in regions of high- and low-dose gradient, respectively. The most common method today to compare two images is the gamma-evaluation method ${ }^{170,171}$, although other methods have also been proposed ${ }^{172,173}$. The gamma-evaluation takes both the dose differences and the DTA into account. The dose criterion should be stated relative to a reference value. Usually this is the maximum or reference dose at the dose specification point (i.e. global) but also the local dose difference could be used (i.e. local). Gamma criteria are indicated as $\mathrm{x} \%, \mathrm{y} \mathrm{mm}$. However, in clinical practice easy to use criteria are needed to quickly evaluate differences between measured and planned dose distributions. Acceptance and rejection criteria can facilitate this process. There is however no consensus on which criteria should be used for specific treatment sites or equipment used.

Various strategies are proposed in the literature with most 2D metrics and criteria based on film dosimetry used to verify the total dose in a plane in a phantom. Film dosimetry is commonly used to detect plan errors, and sometimes also machine errors. Childress et al. ${ }^{174}$ suggested using criteria of $5 \%$ for local dose difference and $3 \mathrm{~mm}$ for DTA. They described the type of errors that could be detected, e.g. beam energy change, wrong collimator rotation or gantry angle. Budgell et al. ${ }^{175}$ used $3 \%$ of the maximum dose and $3 \mathrm{~mm}$ DTA. They focused on the region within the $80 \%$ isodose surface, but switched to $4 \%, 4 \mathrm{~mm}$ criteria if large deviations were visible. The ESTRO QUASIMODO project ${ }^{176}$ used $4 \%, 3 \mathrm{~mm}$ and looked at median and $95 \%$ percentile. Another example of pass/fail criteria for IMRT pre-treatment verification using film dosimetry was presented by Stock et al. ${ }^{177}$, based on the percentage of points with a gamma value larger than a threshold value and the average values of the gamma points in a specific region, using a $3 \%$ dose difference to a reference point and a DTA of $3 \mathrm{~mm}$. If the acceptance criteria were not met, they recommended looking at dose profiles and dose difference maps or at additional parameters such as the gamma angle. This parameter describes whether the dose or the DTA-criterion is dominant.

Spezi et al. ${ }^{178}$ described the use of gamma-area and gamma-volume histograms to analyse 2D and multiple 2D gamma evaluations, respectively, using 3\%, $3 \mathrm{~mm}$ criteria. For all of these studies the final interpretation, if the criteria were not met, was still made by an expert, who decided to accept or reject the dose distribution. Therefore the gamma index has been used more to alert the user to discrepancies, rather than as a strict set of pass/fail criteria.

McDermott et al. ${ }^{162}$ presented criteria and acceptance levels for field-by-field comparisons of multiple fractions. Composite images for each field were made by selecting the minimum, median and 'low' - half-way between minimum and median gamma value per pixel, over mulitple fractions ( 1 to 5 ), in order to filter out random errors. They also introduced 4 levels of 'alert criteria', from very easy to easy, medium and strict based on the average, maximum gamma value and the percentage of points 
exceeding unity. The various alert criteria, and the number of fractions combined, were tested to develop a decision tree to distinguish between systematic and random errors, and minimise false positive and false negative results. The optimum result required a medium level alert criterion, based on combining 'low' gamma values for measurements of 3 fractions for prostate IMRT treatments.

Such decision trees can facilitate the interpretation of differences between dose distributions and the implementation in automatic analysis. Van Zijtveld et al. ${ }^{179}$ implemented such a decision tree for the automatic evaluation of gamma images, both $3 \%, 3 \mathrm{~mm}$ global and 3\%, $3 \mathrm{~mm}$ local, acquired for IMRT pre-treatment verification of the treatment fields delivered and measured using the EPID. They included not only simple metrics such as the number of points failing the gamma evaluation but also incorporated more advanced features of the image such as the size of the area where differences are visible. They suggested that for beam-by-beam analysis the local dose difference criterion should be used to prevent differences in low dose areas from contributing to unacceptable discrepancies in the total plan.

A single solution to what criteria should be used in clinical practice is difficult. Most centres use a gamma evaluation with a dose criterion of $3 \%$ of the maximum or reference dose combined with a DTA criterion of $3 \mathrm{~mm}$, and pose restrictions on metrics based on gamma statistics over a specified area or volume, such as the average gamma value, the maximum gamma value or the proportion of points with a gamma value above a certain threshold value. However, acceptance criteria for the percentage of points not fulfilling these restrictions greatly differ between various centres, treatment techniques and treatment sites. The main reason for these differences is that the level of accuracy that must be achieved should be carefully balanced between the rate of false positive errors (increasing workload unnecessarily) and false negative errors (failing to detect clinically relevant discrepancies).

With the emerging methods that can reconstruct dose in 3D, another interesting point is how these dose distributions need to be compared. Two strategies can be performed. First, a 'standard' verification procedure using the gamma evaluation or dose-difference maps can be performed: a verification of the two data sets at a selected slice, multiple 2D slices or even a gamma evaluation for the entire volume ${ }^{180}$. A second strategy is to analyse the dose distribution in terms of anatomical structures or a region-of-interest-based comparison: Dose-area histograms for $2 \mathrm{D}$ verification or dose-volume histograms for 3D verification methods. The latter strategy might be preferable because a calculation of the parameters in terms of patient-related characteristics can be performed: isodose display on top of the structures of interest, mean dose to the tumour or in the normal tissue, or eventually, a reconstructed dosevolume histogram to get a quick idea of the total treatment plan. This way of verification would ease the process of interpretation of possible differences to the judgement of the verified plan in terms of a 'normal' treatment plan evaluation.

Several examples are given in the literature of errors in dose delivery observed by using EPID measurements in combination with a 3D dose reconstruction method. Van Zijtveld et al. ${ }^{121}$ described the impact of a malfunctioning leaf (machine error), picked up during pre-treatment verification, on the $3 \mathrm{D}$ dose distribution inside the patient. Malfunctioning of one of the leaves caused a difference between measured and predicted portal dose image of about $10 \%$ in one patient. Due to the combination of 
the different treatment fields and the large size of the tumour, the dose differences reduced to $5 \%$ in the reconstructed $3 \mathrm{D}$ dose distribution. They therefore concluded that for clinical evaluation one cannot rely on analysing only the DVH. Proper local dose evaluation is important as well, for example by using automatic slice-by-slice comparison. Another example is given by Chen et al. ${ }^{165}$ describing the in vivo verification of the dose delivered to a patient. The authors measured the dose behind the patient with an EPID and converted the image data to dose values inside the patient using a set of megavoltage cone-beam CT scans. They found differences due to the changed patient anatomy during the course of treatment.

\section{Clinical experience}

Routine clinical use of EPIDs for dosimetry has been restricted to a very few centres until recently. This was partly due to the absence of commercial solutions, the limited use of EPIDs for set-up verification and the lack of demand for patient-specific dose verification. With the introduction of IMRT, these aspects have received much more attention than in the past. The geometric accuracy of patient positioning is very important for IMRT techniques with steep dose gradients between target volumes and organs at risk, thus accelerating the introduction in the clinic of efficient and accurate use of EPIDs to replace film for portal imaging. Furthermore, the need for patientspecific IMRT dose verification also stimulated many groups to investigate the use of EPID dosimetry. Finally many groups realised that EPIDs could be used to perform in vivo dosimetry in a relatively simple way during IMRT treatments, without much additional time at the accelerator. In this section we will summarise the results of clinical studies applying the methods outlined in the previous sections.

The group at the Erasmus Medical Centre - Daniel den Hoed Cancer Center in Rotterdam (The Netherlands) has extensive experience with clinical use of camera-based EPIDs for dosimetric verification of patient treatments, as outlined in the previous sections. In their first paper on clinical use, they reported their experience with in vivo dosimetry based on EPID images acquired during treatment of 10 prostate cancer patients. ${ }^{142}$ EPID images were acquired for all fields during each fraction. The resulting measured portal dose images were compared with the portal dose images predicted with their model, which uses the planning CT scan of the patient. The difference between the average measured and predicted on-axis portal dose was $0.3 \pm 2.1 \%$ (1SD) for the lateral fields and $0.7 \pm 3.4 \%$ (1SD) for the anterior fields. Off-axis differences between measured and predicted dose values were often much larger, up to $15 \%$, and were caused by frequently occurring gas pockets in the rectum during treatment or CT scanning. In a larger group of 115 prostate patients, the dose at $5 \mathrm{~cm}$ depth in the patient, $D_{5}$, was derived from EPID measurements for each treatment beam and compared with the dose predicted by the TPS using the empirical relationship between $D_{5}$ and the portal dose on the beam's central axis. ${ }^{127}$ The mean differences between measured and predicted $D_{5}$ values were similar to those observed when using their portal dose image prediction model. The difference between measured and predicted $D_{5}$ values was larger than $5 \%$ for 7 patients. All these patients had relatively large gas pockets ( 3 to $3.5 \mathrm{~cm}$ diameter in the anterior-posterior direction) in the rectum during acquisition of the planning CT scan, which were not present during most of the treat- 
ments. Pre-treatment verification of dynamic IMRT delivery is performed in Rotterdam using camera-based EPIDs in combination with the first model developed by the Rotterdam group ${ }^{181}$, which compares measured with predicted portal dose images. Recently, van Zijtveld et al. ${ }^{179}$ analysed the results of 270 patients treated with dynamic MLC and verified using this method, in combination with a gamma evaluation. For this large patient group they intercepted 4 severe errors; in one case a wrong treatment plan was sent to the accelerator and three times one of the leaves was malfunctioning. The authors also proposed an automated image analysis scheme, as discussed in the former section, by which visual inspection of images could be avoided in $2 / 3$ of the cases.

The group at the Netherlands Cancer Institute - Antoni van Leeuwenhoek Hospital (NKI-AVL) in Amsterdam (The Netherlands) has developed a back-projection model based on EPID measurements mainly with the idea for use as a routine in vivo dosimetry tool. In a pilot study Boellaard et al. ${ }^{160}$ demonstrated the usefulness of their approach to verify the midplane dose during radiotherapy of larynx, breast, lung, and prostate cancer patients. Midplane dose data derived from portal dose measurements were compared with those calculated with a 3D TPS and generally showed good agreement. The reproducibility was within 1\% (1 SD) and agreed within $2.5 \%$ with treatment planning data for most parts of the field. For a few of the prostate and lung treatments, larger deviations were found due to the differences between the actual patient anatomy and the planning CT data, as a result of variable gas filling in the rectum and anatomical changes in the lung. Clinical results for prostate treatments were later reported, where a-Si EPID dosimetry replaced all film and ionisation chamber measurements for IMRT pre-treatment verification from February $2005 .{ }^{161}$ For 20 patient plans, pre-treatment phantom verification was compared with an EPID and an ionisation chamber yielding an average ratio of $1.00 \pm 0.01$ (1 SD) at the isocentre. The same 20 plans were also verified in $2 \mathrm{D}$, with only $1.5 \%$ of the points yielding gamma values larger than unity ( $3 \%$ of $D_{\max }, 3 \mathrm{~mm}$ DTA). Results for 7 out of 20 plans revealed under-dosage in individual fields ranging from $5 \%$ to $16 \%$, occurring along the junction of abutting segments (tongue-and-groove side). The agreement was improved after adjusting an incorrectly set tongue-and-groove width parameter in the TPS. Subsequently, a report detailed the replacement of pre-treatment with in vivo EPID dosimetry for IMRT prostate radiotherapy. ${ }^{162}$ Dose distributions were reconstructed from EPID images, inside the phantom and patient. Planned and EPID isocentre dose values were, on average, within $1 \%(0.01 \mathrm{SD})$ for both pre-treatment and in vivo verification of the total plan. Multiple in vivo fractions were combined by assessing gamma images, and showed that information obtained in three fractions rendered similar results as pretreatment verification. Additional time for verification was about $2.5 \mathrm{~h}$ per plan for pre-treatment checks and $15 \mathrm{~min}+10 \mathrm{~min} /$ fraction for in vivo dosimetry.

Researchers at the Royal Marsden Hospital in London (UK) have developed a model for using EPID measurements of the tangential fields to construct a thickness model of the patient's breast. Using this information IMRT beams were designed to improve dose homogeneity in the breast by reducing the high-dose region. ${ }^{182}$ This model was an extension of an earlier developed method ${ }^{39}$ to design physical compensators to deliver IMRT to breast cancer patients. Their EPID dosimetry model was the basis of a 
randomized clinical trial in which 300 patients were randomized to evaluate the results of improved dose uniformity on treatment cosmetic outcome. ${ }^{183}$

At the MAASTRO Clinic in Maastricht (The Netherlands) pre-treatment verification and in vivo dosimetry using EPIDs has been performed since 2004 for all patients treated with curative intent with photon beams. They acquired and analysed approximately 32,500 images of about 2500 patients during a period of 24 months. Radiation therapy technologists perform both the measurements and the analysis by using inhouse developed software. Calibrated camera-based EPIDs were used to measure the central field dose, which is compared with a dose prediction at the EPID level. ${ }^{44,62}$ For almost 9000 treatment fields, they found a small mean dose difference of $0.1 \pm 2.4 \%$ (1 SD) and several errors such as wrong manual entering of treatment parameters, the omission of a shielding block and an error in the machine output. For treatment dosimetry, transit dose data were calculated using patient transmission and scatter, and compared with measured values. Furthermore, measured transit dose data are back-projected to a dose value at $5 \mathrm{~cm}$ depth in water $\left(D_{5}\right)$ and directly compared with $D_{5}$ calculated by the TPS. Patient related delivery errors reported were often due to set-up errors and organ motion, initiating further investigation of error sources. Imaging during treatment combined with set-up correction protocols is necessary to decrease dose differences, particularly in the case of breast, lung, and head and neck treatments.

In Bellinzona (Switzerland) results for pre-treatment EPID verification of 44 static IMRT patient plans were reported. ${ }^{110}$ With $9.9 \%$ of points within the measured fields beyond a gamma value of unity ( $3 \%$ of $D_{\max }, 3 \mathrm{~mm}$ DTA), they concluded that the technique was feasible, dosimetrically accurate and recommended for large-scale implementation.

A group in Rome (Italy) ${ }^{130}$ used an a-Si EPID for verifying the dose at the isocentre for a group of 10 patients treated with conformal techniques in the pelvic region. A dosimetric module of a commercial software tool was used to convert the transmitted signal of 25 pixels around the centre of the EPID into dose values, which was then used to determine the dose at the isocentre using a model based on a series of phantom measurements. The authors report that the use of EPIDs strongly reduced the workload of their in vivo dosimetry procedure compared to their previous method in which a small ionisation chamber, positioned on the EPID, was used.

\section{DISCUSSION AND CONCLUSIONS}

The use of EPIDs for dosimetry has matured, and currently a variety of reliable and accurate dose verification methods are used in clinical practice, but this is not yet widespread. Quality control of a linear accelerator, pre-treatment and treatment dose verification, as well as in vivo dosimetry methods using EPIDs are now routinely in use in a growing number of clinics. First it has been shown by several groups that for IMRT verification, EPID dosimetry can replace the more cumbersome film and ionisation chamber measurements. From these studies it can be concluded that one set of EPID measurements is sufficient to check an IMRT plan, making it a useful, efficient and 
accurate dosimetric tool. Furthermore, there is a growing demand to verify that the planned dose distribution is indeed correctly delivered to the patient. For that purpose treatment dosimetry is an essential tool, and EPID dosimetry has shown to be a better alternative to other methods that are limited to point dose information, particularly during IMRT treatments. Once the EPID is calibrated for dosimetry, treatment dosimetry performed with EPIDs is a fast procedure compared to pre-treatment verification because the images are acquired during treatment time (for some methods an additional set of images may be required). The only extra time is needed for analysis, which can be automated. Since portal imaging is generally performed by radiation therapy technologists at the treatment machine, IMRT dose verification based on EPID images acquired during treatment only requires additional input of physicists or radiation oncologists if criteria for acceptance are not met, as for the routine use of portal imaging for patient set-up verification.

As radiotherapy becomes more complicated, both in vivo dosimetry and geometry verification in three dimensions using image-guided radiotherapy (IGRT) methods will become necessary. Also over longer treatment times, anatomical changes such as weight loss, flexing of the neck and variation in lung density, occur frequently, resulting in dose delivery variation over a series of fractions. Methods to integrate 3D in vivo dosimetry and IGRT procedures, such as the use of $\mathrm{kV}$ or MV cone-beam CT, are therefore under development. IGRT procedures using a cone-beam scan made on the treatment day for dose reconstruction, as well as for re-contouring volumes of interest, would improve the accuracy of in vivo dosimetry for many treatment sites. A challenge will be to implement these procedures in clinical routine; i.e. finding answers for questions such as when is a deviation in dose clinically unacceptable, when should a patient be re-planned, and how should dose variations in deformed volumes be taken into account in an efficient way.

EPID dosimetry is evolving now to an integrated part in the total chain of verification procedures that are implemented in a radiotherapy department. It provides a safety net for advanced treatments as well as a full account of the dose delivered to specific volumes, allowing adaptation of the treatment from the original plan if necessary. Finally it should be noted that large-scale clinical implementation of EPID dosimetry is dependent upon commercial availability. Current models and software are developed by research groups and are mainly limited to academic centres. Commercial solutions should be easy to implement, simple, robust and efficient in their daily use, and be sufficiently accurate for the purpose they are serving.

\section{ACKNOWLEDGEMENTS}

Two authors, L.N. McDermott and M. Wendling, would like to acknowledge the financial support of the Technology Foundation STW (Project no. 7184). The authors are also grateful to colleagues at MAASTRO and NKI-AVL for contribution to discussions about the clinical role of EPID dosimetry in a modern radiotherapy department. 


\section{REFERENCES}

${ }^{1}$ M.G. Herman, "Clinical use of electronic portal imaging," Semin Radiat Oncol 15, 157-167 (2005).

${ }^{2}$ M.G. Herman, J.M. Balter, D.A. Jaffray, K.P. McGee, P. Munro, S. Shalev, M. Van Herk, and J.W. Wong, "Clinical use of electronic portal imaging: report of AAPM Radiation Therapy Committee Task Group 58," Med Phys 28, $712-737$ (2001).

${ }^{3}$ M.G. Herman, J.J. Kruse, and C.R. Hagness, "Guide to clinical use of electronic portal imaging," J Appl Clin Med Phys 1, 38-57 (2000).

${ }^{4}$ K.A. Langmack, "Portal imaging," Br J Radiol 74, 789-804 (2001).

${ }^{5}$ L.E. Antonuk, "Electronic portal imaging devices: a review and historical perspective of contemporary technologies and research," Phys Med Biol 47, R31-65 (2002).

${ }^{6} \mathrm{C}$. De Wagter, QA-QC of IMRT: European Perspective, in Image-Guided IMRT, T. Bortfeld, et al., Editors. 2006, Springer-Verlag: Berlin-Heidelberg.

7J.M. Moran, QA-QC of IMRT: American Perspective, in Image-Guided IMRT, T. Bortfeld, et al., Editors. 2006, Springer-Verlag: Berlin-Heidelberg.

${ }^{8}$ T. Wiezorek, N. Banz, M. Schwedas, M. Scheithauer, H. Salz, D. Georg, and T.G. Wendt, "Dosimetric quality assurance for intensity-modulated radiotherapy feasibility study for a filmless approach," Strahlenther Onkol 181, 468-474 (2005).

${ }^{9}$ S. Amerio, A. Boriano, F. Bourhaleb, R. Cirio, M. Donetti, A. Fidanzio, E. Garelli, S. Giordanengo, E. Madon, F. Marchetto, U. Nastasi, C. Peroni, A. Piermattei, C.J. Sanz Freire, A. Sardo, and E. Trevisiol, "Dosimetric characterization of a large area pixel-segmented ionization chamber," Med Phys 31, 414-420 (2004).

${ }^{10}$ E. Spezi, A.L. Angelini, F. Romani, and A. Ferri, "Characterization of a 2D ion chamber array for the verification of radiotherapy treatments," Phys Med Biol 50, 3361-3373 (2005).

${ }^{11}$ D. Letourneau, M. Gulam, D. Yan, M. Oldham, and J.W. Wong, "Evaluation of a 2D diode array for IMRT quality assurance," Radiother Oncol 70, 199-206 (2004).

${ }^{12}$ P.A. Jursinic and B.E. Nelms, "A 2-D diode array and analysis software for verification of intensity modulated radiation therapy delivery," Med Phys 30, 870-879 (2003).

${ }^{13}$ A.F. Monti and G. Frigerio, "Dosimetric verification of 6 and $18 \mathrm{MV}$ intensity modulated photon beams using a dedicated fluoroscopic electronic portal imaging device (EPID)," Radiother Oncol 81, 88-96 (2006).

${ }^{14}$ B.M. Hesse, L. Spies, and B.A. Groh, "Tomotherapeutic portal imaging for radiation treatment verification," Phys Med Biol 43, 3607-3616 (1998).

${ }^{15}$ F. Cremers, T. Frenzel, C. Kausch, D. Albers, T. Schonborn, and R. Schmidt, "Performance of electronic portal imaging devices (EPIDs) used in radiotherapy: image quality and dose measurements," Med Phys 31, 985-996 (2004).

${ }^{16}$ A.L. Boyer, L. Antonuk, A. Fenster, M. Van Herk, H. Meertens, P. Munro, L.E. Reinstein, and J. Wong, "A review of electronic portal imaging devices (EPIDs)," Med Phys 19, 1-16 (1992).

${ }^{17}$ M.C. Kirby and A.G. Glendinning, "Developments in electronic portal imaging systems," $\mathrm{Br} \mathrm{J}$ Radiol 79 Spec No 1, S50-65 (2006).

${ }^{18} \mathrm{M}$. van Herk, "Physical aspects of a liquid-filled ionization chamber with pulsed polarizing voltage," Med Phys 18, 692-702 (1991).

${ }^{19} \mathrm{H}$. Meertens, M. van Herk, J. Bijhold, and H. Bartelink, "First clinical experience with a newly developed electronic portal imaging device," Int J Radiat Oncol Biol Phys 18, 1173-1181 (1990).

${ }^{20} \mathrm{H}$. Meertens, M. van Herk, and J. Weeda, "A liquid ionisation detector for digital radiography of therapeutic megavoltage photon beams," Phys Med Biol 30, 313-321 (1985).

${ }^{21} \mathrm{M}$. van Herk and H. Meertens, "A matrix ionisation chamber imaging device for on-line patient setup verification during radiotherapy," Radiother Oncol 11, 369-378 (1988). 
${ }^{22}$ M. Essers, R. Boellaard, M. van Herk, H. Lanson, and B. Mijnheer, "Transmission dosimetry with a liquid-filled electronic portal imaging device," Int J Radiat Oncol Biol Phys 34, 931-941 (1996).

${ }^{23}$ F.F. Yin, M.C. Schell, and P. Rubin, "Input/output characteristics of a matrix ion-chamber electronic portal imaging device," Med Phys 21, 1447-1454 (1994).

${ }^{24}$ M. Essers, B.R. Hoogervorst, M. van Herk, H. Lanson, and B.J. Mijnheer, "Dosimetric characteristics of a liquid-filled electronic portal imaging device," Int J Radiat Oncol Biol Phys 33, 1265-1272 (1995).

${ }^{25}$ Y. Zhu, X.Q. Jiang, and J. Van Dyk, "Portal dosimetry using a liquid ion chamber matrix: dose response studies," Med Phys 22, 1101-1106 (1995).

${ }^{26}$ R. Boellaard, M. van Herk, and B.J. Mijnheer, "The dose response relationship of a liquid-filled electronic portal imaging device," Med Phys 23, 1601-1611 (1996).

${ }^{27} \mathrm{H}$. Keller, M. Fix, and P. Ruegsegger, "Calibration of a portal imaging device for high-precision dosimetry: a Monte Carlo study," Med Phys 25, 1891-1902 (1998).

${ }^{28} \mathrm{H}$. Parsaei, E. el-Khatib, and R. Rajapakshe, "The use of an electronic portal imaging system to measure portal dose and portal dose profiles," Med Phys 25, 1903-1909 (1998).

${ }^{29}$ J. Chang, G.S. Mageras, C.C. Ling, and W. Lutz, "An iterative EPID calibration procedure for dosimetric verification that considers the EPID scattering factor," Med Phys 28, 2247-2257 (2001).

${ }^{30}$ A. Van Esch, B. Vanstraelen, J. Verstraete, G. Kutcher, and D. Huyskens, "Pre-treatment dosimetric verification by means of a liquid-filled electronic portal imaging device during dynamic delivery of intensity modulated treatment fields," Radiother Oncol 60, 181-190 (2001).

${ }^{31}$ J. Chang, G.S. Mageras, and C.C. Ling, "Evaluation of rapid dose map acquisition of a scanning liquid-filled ionization chamber electronic portal imaging device," Int J Radiat Oncol Biol Phys 55, 1432-1445 (2003).

${ }^{32}$ P.W. Chin, D.G. Lewis, and E. Spezi, "Correction for dose-response variations in a scanning liquid ion chamber EPID as a function of linac gantry angle," Phys Med Biol 49, N93-103 (2004).

${ }^{33}$ R.J. Louwe, R. Tielenburg, K.M. van Ingen, B.J. Mijnheer, and M.B. van Herk, "The stability of liquid-filled matrix ionization chamber electronic portal imaging devices for dosimetry purposes," Med Phys 31, 819-827 (2004).

${ }^{34}$ P. Reich, E. Bezak, M. Mohammadi, and L. Fog, "The prediction of transmitted dose distributions using a 3D treatment planning system," Australas Phys Eng Sci Med 29, 18-29 (2006).

${ }^{35} \mathrm{M}$. Mohammadi and E. Bezak, "Two-dimensional transmitted dose measurements using a scanning liquid ionization chamber EPID," Phys Med Biol 51, 2971-2985 (2006).

${ }^{36}$ K. Tateoka, A. Ouchi, M. Waka, K. Nakata, D. Nagase, K. Shimizume, T. Saikawa, and M. Hareyama, "Dosimetric properties of the liquid ionization chamber electronic portal imaging device (EPID)," Igaku Butsuri 26, 28-38 (2006).

${ }^{37}$ E.J. Morton, W. Swindell, D.G. Lewis, and P.M. Evans, "A linear array, scintillation crystalphotodiode detector for megavoltage imaging," Med Phys 18, 681-691 (1991).

${ }^{38}$ J.R. Symonds-Tayler, M. Partridge, and P.M. Evans, "An electronic portal imaging device for transit dosimetry," Phys Med Biol 42, 2273-2283 (1997).

${ }^{39}$ V.N. Hansen, P.M. Evans, and W. Swindell, "The application of transit dosimetry to precision radiotherapy," Med Phys 23, 713-721 (1996).

${ }^{40}$ M.A. Mosleh-Shirazi, P.M. Evans, W. Swindell, J.R. Symonds-Tayler, S. Webb, and M. Partridge, "Rapid portal imaging with a high-efficiency, large field-of-view detector," Med Phys 25, 2333-2346 (1998). 
${ }^{41}$ V.G. Althof, J.C. de Boer, H. Huizenga, J.C. Stroom, A.G. Visser, and B.N. Swanenburg, "Physical characteristics of a commercial electronic portal imaging device," Med Phys 23, 1845-1855 (1996).

${ }^{42}$ B.J. Heijmen, K.L. Pasma, M. Kroonwijk, V.G. Althof, J.C. de Boer, A.G. Visser, and H. Huizenga, "Portal dose measurement in radiotherapy using an electronic portal imaging device (EPID)," Phys Med Biol 40, 1943-1955 (1995).

${ }^{43}$ K.L. Pasma, M. Kroonwijk, J.C. de Boer, A.G. Visser, and B.J. Heijmen, "Accurate portal dose measurement with a fluoroscopic electronic portal imaging device (EPID) for open and wedged beams and dynamic multileaf collimation," Phys Med Biol 43, 2047-2060 (1998).

${ }^{44}$ S.M. Nijsten, A.W. Minken, P. Lambin, and I.A. Bruinvis, "Verification of treatment parameter transfer by means of electronic portal dosimetry," Med Phys 31, 341-347 (2004).

${ }^{45}$ A.G. Glendinning and D.E. Bonnett, "Dosimetric properties of the Theraview fluoroscopic electronic portal imaging device," Br J Radiol 73, 517-530 (2000).

${ }^{46}$ A.G. Glendinning, S.G. Hunt, and D.E. Bonnett, "Measurement of the response of Gd2O2S:Tb phosphor to 6 MV x-rays," Phys Med Biol 46, 517-530 (2001).

${ }^{47}$ E.M. Franken, J.C. de Boer, J.C. Barnhoorn, and B.J. Heijmen, "Characteristics relevant to portal dosimetry of a cooled CCD camera-based EPID," Med Phys 31, 2549-2551 (2004).

${ }^{48}$ E.M. Franken, J.C. de Boer, and B.J. Heijmen, "A novel approach to accurate portal dosimetry using CCD-camera based EPIDs," Med Phys 33, 888-903 (2006).

${ }^{49}$ J.C. de Boer, B.J. Heijmen, K.L. Pasma, and A.G. Visser, "Characterization of a high-elbow, fluoroscopic electronic portal imaging device for portal dosimetry," Phys Med Biol 45, 197216 (2000).

${ }^{50}$ M. Partridge, P.M. Evans, and J.R. Symonds-Tayler, "Optical scattering in camera-based electronic portal imaging," Phys Med Biol 44, 2381-2396 (1999).

${ }^{51}$ M. Partridge, J.R. Symonds-Tayler, and P.M. Evans, "IMRT verification with a camera-based electronic portal imaging system," Phys Med Biol 45, N183-196 (2000).

${ }^{52}$ M.L. Dirkx and B.J. Heijmen, "Testing of the stability of intensity modulated beams generated with dynamic multileaf collimation, applied to the MM50 racetrack microtron," Med Phys 27, 2701-2707 (2000).

${ }^{53}$ M.L. Dirkx, M. Kroonwijk, J.C. de Boer, and B.J. Heijmen, "Daily dosimetric quality control of the MM50 Racetrack Microtron using an electronic portal imaging device," Radiother Oncol 37, 55-60 (1995).

${ }^{54}$ L.E. Antonuk, Y. El-Mohri, W. Huang, K.W. Jee, J.H. Siewerdsen, M. Maolinbay, V.E. Scarpine, H. Sandler, and J. Yorkston, "Initial performance evaluation of an indirect-detection, active matrix flat-panel imager (AMFPI) prototype for megavoltage imaging," Int J Radiat Oncol Biol Phys 42, 437-454 (1998).

${ }^{55}$ L.E. Antonuk, J. Yorkston, W. Huang, J.H. Siewerdsen, J.M. Boudry, Y. el-Mohri, and M.V. Marx, "A real-time, flat-panel, amorphous silicon, digital x-ray imager," Radiographics 15, 993 1000 (1995).

${ }^{56}$ L.N. McDermott, R.J. Louwe, J.J. Sonke, M.B. van Herk, and B.J. Mijnheer, "Dose-response and ghosting effects of an amorphous silicon electronic portal imaging device," Med Phys 31, 285-295 (2004).

${ }^{57} \mathrm{P}$. Winkler and D. Georg, "An intercomparison of 11 amorphous silicon EPIDs of the same type: implications for portal dosimetry," Phys Med Biol 51, 4189-4200 (2006).

${ }^{58}$ L. Parent, J. Seco, P.M. Evans, A. Fielding, and D.R. Dance, "Monte Carlo modelling of a-Si EPID response: the effect of spectral variations with field size and position," Med Phys 33, 45274540 (2006).

${ }^{59}$ P. Winkler, A. Hefner, and D. Georg, "Dose-response characteristics of an amorphous silicon EPID," Med Phys 32, 3095-3105 (2005). 
${ }^{60}$ L. Parent, A.L. Fielding, D.R. Dance, J. Seco, and P.M. Evans, "Amorphous silicon EPID calibration for dosimetric applications: comparison of a method based on Monte Carlo prediction of response with existing techniques," Phys Med Biol 52, 3351-3368 (2007).

${ }^{61}$ J. Chen, C.F. Chuang, O. Morin, M. Aubin, and J. Pouliot, "Calibration of an amorphous-silicon flat panel portal imager for exit-beam dosimetry," Med Phys 33, 584-594 (2006).

${ }^{62}$ S.M. Nijsten, W.J. van Elmpt, M. Jacobs, B.J. Mijnheer, A.L. Dekker, P. Lambin, and A.W. Minken, "A global calibration model for a-Si EPIDs used for transit dosimetry," Med Phys 34, 3872-3884 (2007).

${ }^{63}$ P.B. Greer, "Correction of pixel sensitivity variation and off-axis response for amorphous silicon EPID dosimetry," Med Phys 32, 3558-3568 (2005).

${ }^{64}$ A. Van Esch, T. Depuydt, and D.P. Huyskens, "The use of an aSi-based EPID for routine absolute dosimetric pre-treatment verification of dynamic IMRT fields," Radiother Oncol 71, 223234 (2004).

${ }^{65}$ P.B. Greer and C.C. Popescu, "Dosimetric properties of an amorphous silicon electronic portal imaging device for verification of dynamic intensity modulated radiation therapy," Med Phys 30, 1618-1627 (2003).

${ }^{66} \mathrm{C}$. Kirkby and R. Sloboda, "Consequences of the spectral response of an a-Si EPID and implications for dosimetric calibration," Med Phys 32, 2649-2658 (2005).

${ }^{67}$ B.M. McCurdy, K. Luchka, and S. Pistorius, "Dosimetric investigation and portal dose image prediction using an amorphous silicon electronic portal imaging device," Med Phys 28, 911 924 (2001).

${ }^{68}$ J.V. Siebers, J.O. Kim, L. Ko, P.J. Keall, and R. Mohan, "Monte Carlo computation of dosimetric amorphous silicon electronic portal images," Med Phys 31, 2135-2146 (2004).

${ }^{69}$ E.E. Grein, R. Lee, and K. Luchka, "An investigation of a new amorphous silicon electronic portal imaging device for transit dosimetry," Med Phys 29, 2262-2268 (2002).

${ }^{70} \mathrm{C}$. Kirkby and R. Sloboda, "Comprehensive Monte Carlo calculation of the point spread function for a commercial a-Si EPID," Med Phys 32, 1115-1127 (2005).

${ }^{71}$ R.J. Louwe, L.N. McDermott, J.J. Sonke, R. Tielenburg, M. Wendling, M.B. van Herk, and B.J. Mijnheer, "The long-term stability of amorphous silicon flat panel imaging devices for dosimetry purposes," Med Phys 31, 2989-2995 (2004).

${ }^{72}$ Y. El-Mohri, L.E. Antonuk, J. Yorkston, K.W. Jee, M. Maolinbay, K.L. Lam, and J.H. Siewerdsen, "Relative dosimetry using active matrix flat-panel imager (AMFPI) technology," Med Phys 26, 1530-1541 (1999).

${ }^{73}$ M. Partridge, B.-M. Hesse, and L. Müller, "A performance comparison of direct- and indirectdetection flat-panel imagers," Nucl.Instrum. Methods Phys.Res.A 484, 351-363 (2002).

${ }^{74}$ A.E. Schach von Wittenau, C.M. Logan, M.B. Aufderheide, 3rd, and D.M. Slone, "Blurring artifacts in megavoltage radiography with a flat-panel imaging system: comparison of Monte Carlo simulations with measurements," Med Phys 29, 2559-2570 (2002).

${ }^{75}$ P.B. Greer, "Off-axis dose response characteristics of an amorphous silicon electronic portal imaging device," Med Phys 34, 3815-3824 (2007).

${ }^{76}$ M. Wendling, R.J. Louwe, L.N. McDermott, J.J. Sonke, M. van Herk, and B.J. Mijnheer, "Accurate two-dimensional IMRT verification using a back-projection EPID dosimetry method," Med Phys 33, 259-273 (2006).

${ }^{77}$ C. Talamonti, M. Casati, and M. Bucciolini, "Pretreatment verification of IMRT absolute dose distributions using a commercial a-Si EPID," Med Phys 33, 4367-4378 (2006).

${ }^{78}$ L.N. McDermott, S.M. Nijsten, J.J. Sonke, M. Partridge, M. van Herk, and B.J. Mijnheer, "Comparison of ghosting effects for three commercial a-Si EPIDs," Med Phys 33, 2448-2451 (2006).

${ }^{79}$ J.H. Siewerdsen and D.A. Jaffray, "A ghost story: spatio-temporal response characteristics of an indirect-detection flat-panel imager," Med Phys 26, 1624-1641 (1999). 
${ }^{80}$ G.J. Budgell, Q. Zhang, R.J. Trouncer, and R.I. Mackay, "Improving IMRT quality control efficiency using an amorphous silicon electronic portal imager," Med Phys 32, 3267-3278 (2005).

${ }^{81}$ R. Boellaard, M. van Herk, and B.J. Mijnheer, "A convolution model to convert transmission dose images to exit dose distributions," Med Phys 24, 189-199 (1997).

${ }^{82}$ J. Chang, G.S. Mageras, C.S. Chui, C.C. Ling, and W. Lutz, "Relative profile and dose verification of intensity-modulated radiation therapy," Int J Radiat Oncol Biol Phys 47, 231-240 (2000).

${ }^{83}$ P.W. Chin, E. Spezi, and D.G. Lewis, "Monte Carlo simulation of portal dosimetry on a rectilinear voxel geometry: a variable gantry angle solution," Phys Med Biol 48, N231-238 (2003).

${ }^{84}$ S.J. Baker, G.J. Budgell, and R.I. MacKay, "Use of an amorphous silicon electronic portal imaging device for multileaf collimator quality control and calibration," Phys Med Biol 50, 13771392 (2005).

${ }^{85}$ A. Curtin-Savard and E.B. Podgorsak, "An electronic portal imaging device as a physics tool," Med Dosim 22, 101-105 (1997).

${ }^{86}$ S.C. Vieira, R.A. Bolt, M.L. Dirkx, A.G. Visser, and B.J. Heijmen, "Fast, daily linac verification for segmented IMRT using electronic portal imaging," Radiother Oncol 80, 86-92 (2006).

${ }^{87}$ G.J. Budgell, R. Zhang, and R.I. Mackay, "Daily monitoring of linear accelerator beam parameters using an amorphous silicon EPID," Phys Med Biol 52, 1721-1733 (2007).

${ }^{88}$ J. Chang, C.H. Obcemea, J. Sillanpaa, J. Mechalakos, and C. Burman, "Use of EPID for leaf position accuracy QA of dynamic multi-leaf collimator (DMLC) treatment," Med Phys 31, 2091-2096 (2004).

${ }^{89} \mathrm{G}$. Liu, T. van Doorn, and E. Bezak, "Assessment of flatness and symmetry of megavoltage x-ray beam with an electronic portal imaging device (EPID)," Australas Phys Eng Sci Med 25, 5866 (2002).

${ }^{90} \mathrm{P}$. Metcalfe, P. Tangboonduangjit, and P. White, "Intensity-modulated radiation therapy: overlapping co-axial modulated fields," Phys Med Biol 49, 3629-3637 (2004).

${ }^{91}$ M.J. Williams and P. Metcalfe, "Verification of a rounded leaf-end MLC model used in a radiotherapy treatment planning system," Phys Med Biol 51, N65-78 (2006).

${ }^{92}$ L.S. Ploeger, M.H. Smitsmans, K.G. Gilhuijs, and M. van Herk, "Accurate measurement of the dynamic response of a scanning electronic portal imaging device," Med Phys 28, 310-316 (2001).

${ }^{93}$ J.I. Prisciandaro, M.G. Herman, and J.J. Kruse, "Utilizing an electronic portal imaging device to monitor light and radiation field congruence," J Appl Clin Med Phys 4, 315-320 (2003).

${ }^{94}$ L. Xing and J.G. Li, "Computer verification of fluence map for intensity modulated radiation therapy," Med Phys 27, 2084-2092 (2000).

${ }^{95}$ Y. Yang and L. Xing, "Quantitative measurement of MLC leaf displacements using an electronic portal image device," Phys Med Biol 49, 1521-1533 (2004).

${ }^{96}$ S.S. Samant, W. Zheng, N.A. Parra, J. Chandler, A. Gopal, J. Wu, J. Jain, Y. Zhu, and M. Sontag, "Verification of multileaf collimator leaf positions using an electronic portal imaging device," Med Phys 29, 2900-2912 (2002).

${ }^{97}$ L. Parent, J. Seco, P.M. Evans, D.R. Dance, and A. Fielding, "Evaluation of two methods of predicting MLC leaf positions using EPID measurements," Med Phys 33, 3174-3182 (2006).

${ }^{98}$ S.C. Vieira, M.L. Dirkx, K.L. Pasma, and B.J. Heijmen, "Fast and accurate leaf verification for dynamic multileaf collimation using an electronic portal imaging device," Med Phys 29, 2034-2040 (2002).

${ }^{99}$ M. Partridge, P.M. Evans, M. van Herk, L.S. Ploeger, G.J. Budgell, and H.V. James, "Leaf position verification during dynamic beam delivery: a comparison of three applications using electronic portal imaging," Med Phys 27, 1601-1609 (2000).

${ }^{100}$ L.S. Ploeger, M.H. Smitsmans, K.G. Gilhuijs, and M. van Herk, "A method for geometrical verification of dynamic intensity modulated radiotherapy using a scanning electronic portal imaging device," Med Phys 29, 1071-1079 (2002). 
${ }^{101}$ J.J. Sonke, L.S. Ploeger, B. Brand, M.H. Smitsmans, and M. van Herk, "Leaf trajectory verification during dynamic intensity modulated radiotherapy using an amorphous silicon flat panel imager," Med Phys 31, 389-395 (2004).

${ }^{102}$ H.V. James, S. Atherton, G.J. Budgell, M.C. Kirby, and P.C. Williams, "Verification of dynamic multileaf collimation using an electronic portal imaging device," Phys Med Biol 45, 495-509 (2000).

${ }^{103}$ W. Li, J.V. Siebers, and J.A. Moore, "Using fluence separation to account for energy spectra dependence in computing dosimetric a-Si EPID images for IMRT fields," Med Phys 33, 4468-4480 (2006).

${ }^{104}$ K. Chytyk and B.M. McCurdy, "Investigation of tilted dose kernels for portal dose prediction in a-Si electronic portal imagers," Med Phys 33, 3333-3339 (2006).

${ }^{105} \mathrm{C}$. Yeboah and S. Pistorius, "Monte Carlo studies of the exit photon spectra and dose to a metal/phosphor portal imaging screen," Med Phys 27, 330-339 (2000).

${ }^{106}$ J. Chang and C.C. Ling, "Using the frame averaging of aS500 EPID for IMRT verification," J Appl Clin Med Phys 4, 287-299 (2003).

${ }^{107}$ J.M. Kapatoes, G.H. Olivera, K.J. Ruchala, and T.R. Mackie, "On the verification of the incident energy fluence in tomotherapy IMRT," Phys Med Biol 46, 2953-2965 (2001).

${ }^{108}$ O.A. Zeidan, J.G. Li, M. Ranade, A.M. Stell, and J.F. Dempsey, "Verification of step-and-shoot IMRT delivery using a fast video-based electronic portal imaging device," Med Phys 31, 463-476 (2004).

${ }^{109}$ B. Warkentin, S. Steciw, S. Rathee, and B.G. Fallone, "Dosimetric IMRT verification with a flatpanel EPID," Med Phys 30, 3143-3155 (2003).

${ }^{110}$ L. Cozzi, A. Fogliata, and G. Nicolini, "Pre-treatment verification of intensity modulated photon beams with films and electronic portal imaging--two years of clinical experience," Z Med Phys 14, 239-250 (2004).

${ }^{111}$ J.M. Moran, D.A. Roberts, T.S. Nurushev, L.E. Antonuk, Y. El-Mohri, and B.A. Fraass, "An Active Matrix Flat Panel Dosimeter (AMFPD) for in-phantom dosimetric measurements," Med Phys 32, 466-472 (2005).

${ }^{112}$ G. Nicolini, A. Fogliata, E. Vanetti, A. Clivio, and L. Cozzi, "GLAaS: an absolute dose calibration algorithm for an amorphous silicon portal imager. Applications to IMRT verifications," Med Phys 33, 2839-2851 (2006).

${ }^{113}$ B.M. Hesse, S. Nill, T. Tücking, and U. Oelfke, "3D in vivo dosimetry by means of entrance dosimetry for dose guided radiotherapy," Radiother Oncol 81, S46 (2006).

${ }^{114}$ B. Poppe, C. Thieke, D. Beyer, R. Kollhoff, A. Djouguela, A. Ruhmann, K.C. Willborn, and D. Harder, "DAVID--a translucent multi-wire transmission ionization chamber for in vivo verification of IMRT and conformal irradiation techniques," Phys Med Biol 51, 1237-1248 (2006).

${ }^{115} \mathrm{~W}$. Ansbacher, "Three-dimensional portal image-based dose reconstruction in a virtual phantom for rapid evaluation of IMRT plans," Med Phys 33, 3369-3382 (2006).

${ }^{116}$ W.J. van Elmpt, S.M. Nijsten, R.F. Schiffeleers, A.L. Dekker, B.J. Mijnheer, P. Lambin, and A.W. Minken, "A Monte Carlo based three-dimensional dose reconstruction method derived from portal dose images," Med Phys 33, 2426-2434 (2006).

${ }^{117}$ W.J. van Elmpt, S.M. Nijsten, A.L. Dekker, B.J. Mijnheer, and P. Lambin, "Treatment verification in the presence of inhomogeneities using EPID-based three-dimensional dose reconstruction," Med Phys 34, 2816-2826 (2007).

${ }^{118}$ W. van Elmpt, S. Nijsten, B. Mijnheer, A. Dekker, and P. Lambin, "The next step in patientspecific QA: 3D dose verification of conformal and intensity-modulated RT based on EPID dosimetry and Monte Carlo dose calculations," Radiother Oncol 86, 86-92 (2008). 
${ }^{119}$ W.D. Renner, K. Norton, and T. Holmes, "A method for deconvolution of integrated electronic portal images to obtain incident fluence for dose reconstruction," J Appl Clin Med Phys 6, 22-39 (2005).

${ }^{120}$ S. Steciw, B. Warkentin, S. Rathee, and B.G. Fallone, "Three-dimensional IMRT verification with a flat-panel EPID," Med Phys 32, 600-612 (2005).

${ }^{121}$ M. van Zijtveld, M.L. Dirkx, H.C. de Boer, and B.J. Heijmen, "3D dose reconstruction for clinical evaluation of IMRT pretreatment verification with an EPID," Radiother Oncol 82, 201-207 (2007).

${ }^{122}$ M. van Zijtveld, M. Dirkx, and B. Heijmen, "Correction of conebeam CT values using a planning CT for derivation of the "dose of the day"," Radiother Oncol 85, 195-200 (2007).

${ }^{123}$ T.R. McNutt, T.R. Mackie, P. Reckwerdt, and B.R. Paliwal, "Modeling dose distributions from portal dose images using the convolution/superposition method," Med Phys 23, 1381-1392 (1996).

${ }^{124}$ T.R. McNutt, T.R. Mackie, and B.R. Paliwal, "Analysis and convergence of the iterative convolution/superposition dose reconstruction technique for multiple treatment beams and tomotherapy," Med Phys 24, 1465-1476 (1997).

${ }^{125} \mathrm{G}$. Leunens, J. Van Dam, A. Dutreix, and E. van der Schueren, "Quality assurance in radiotherapy by in vivo dosimetry. 1 . Entrance dose measurements, a reliable procedure," Radiother Oncol 17, 141-151 (1990).

${ }^{126}$ M. Essers, J.H. Lanson, G. Leunens, T. Schnabel, and B.J. Mijnheer, "The accuracy of CT-based inhomogeneity corrections and in vivo dosimetry for the treatment of lung cancer," Radiother Oncol 37, 199-208 (1995).

${ }^{127}$ K.L. Pasma, M. Kroonwijk, S. Quint, A.G. Visser, and B.J. Heijmen, "Transit dosimetry with an electronic portal imaging device (EPID) for 115 prostate cancer patients," Int J Radiat Oncol Biol Phys 45, 1297-1303 (1999).

${ }^{128}$ S.M. Nijsten, B.J. Mijnheer, A.L. Dekker, P. Lambin, and A.W. Minken, "Routine individualised patient dosimetry using electronic portal imaging devices," Radiother Oncol 83, 65-75 (2007).

${ }^{129}$ M. D'Andrea, G. Laccarino, S. Carpino, L. Strigari, and M. Benassi, "Primary photon fluence extraction from portal images acquired with an amorphous silicon flat panel detector: experimental determination of a scatter filter," J Exp Clin Cancer Res 26, 125-132 (2007).

${ }^{130}$ A. Piermattei, A. Fidanzio, G. Stimato, L. Azario, L. Grimaldi, G. D'Onofrio, S. Cilla, M. Balducci, M.A. Gambacorta, N. Di Napoli, and N. Cellini, "In vivo dosimetry by an aSi-based EPID," Med Phys 33, 4414-4422 (2006).

${ }^{131}$ A. Piermattei, A. Fidanzio, L. Azario, L. Grimaldi, G. D'Onofrio, S. Cilla, G. Stimato, D. Gaudino, S. Ramella, R. D'Angelillo, F. Cellini, L. Trodella, A. Russo, L. Iadanza, S. Zucca, V. Fusco, N. Di Napoli, M.A. Gambacorta, M. Balducci, N. Cellini, F. Deodato, G. Macchia, and A.G. Morganti, "Application of a practical method for the isocenter point in vivo dosimetry by a transit signal," Phys Med Biol 52, 5101-5117 (2007).

${ }^{132}$ K.L. Pasma, B.J. Heijmen, M. Kroonwijk, and A.G. Visser, "Portal dose image (PDI) prediction for dosimetric treatment verification in radiotherapy. I. An algorithm for open beams," Med Phys 25, 830-840 (1998).

${ }^{133}$ X. He, A. Van Esch, R. Reymen, and D. Huyskens, "Evaluation of an electronic portal imaging device for transit dosimetry," Acta Oncol 38, 591-596 (1999).

${ }^{134}$ M.L. Dirkx, J.C. de Boer, and B.J. Heijmen, "Improvement of radiotherapy treatment delivery accuracy using an electronic portal imaging device," Radiat Prot Dosimetry 121, 70-79 (2006).

${ }^{135}$ W.J. van Elmpt, S.M. Nijsten, B.J. Mijnheer, and A.W. Minken, "Experimental verification of a portal dose prediction model," Med Phys 32, 2805-2818 (2005). 
${ }^{136}$ B.M. McCurdy and S. Pistorius, "Photon scatter in portal images: accuracy of a fluence based pencil beam superposition algorithm," Med Phys 27, 913-922 (2000).

${ }^{137}$ B.M. McCurdy and S. Pistorius, "Photon scatter in portal images: physical characteristics of pencil beam kernels generated using the EGS Monte Carlo code," Med Phys 27, 312-320 (2000).

${ }^{138}$ B.M. McCurdy and S. Pistorius, "A two-step algorithm for predicting portal dose images in arbitrary detectors," Med Phys 27, 2109-2116 (2000).

${ }^{139}$ C.V. Dahlgren, A. Ahnesjo, A. Montelius, and G. Rikner, "Portal dose image verification: formalism and application of the collapsed cone superposition method," Phys Med Biol 47, 4371-4387 (2002).

${ }^{140}$ C.V. Dahlgren, K. Eilertsen, T.D. Jorgensen, and A. Ahnesjo, "Portal dose image verification: the collapsed cone superposition method applied with different electronic portal imaging devices," Phys Med Biol 51, 335-349 (2006).

${ }^{141}$ K.L. Pasma, S.C. Vieira, and B.J. Heijmen, "Portal dose image prediction for dosimetric treatment verification in radiotherapy. II. An algorithm for wedged beams," Med Phys 29, 925931 (2002).

${ }^{142}$ M. Kroonwijk, K.L. Pasma, S. Quint, P.C. Koper, A.G. Visser, and B.J. Heijmen, "In vivo dosimetry for prostate cancer patients using an electronic portal imaging device (EPID); demonstration of internal organ motion," Radiother Oncol 49, 125-132 (1998).

${ }^{143}$ S.C. Vieira, M.L. Dirkx, B.J. Heijmen, and H.C. de Boer, "SIFT: a method to verify the IMRT fluence delivered during patient treatment using an electronic portal imaging device," Int J Radiat Oncol Biol Phys 60, 981-993 (2004).

${ }^{144}$ A.L. Fielding, P.M. Evans, and C.H. Clark, "Verification of patient position and delivery of IMRT by electronic portal imaging," Radiother Oncol 73, 339-347 (2004).

${ }^{145}$ E. Spezi and D.G. Lewis, "Full forward Monte Carlo calculation of portal dose from MLC collimated treatment beams," Phys Med Biol 47, 377-390 (2002).

${ }^{146}$ L. Spies, P.M. Evans, M. Partridge, V.N. Hansen, and T. Bortfeld, "Direct measurement and analytical modeling of scatter in portal imaging," Med Phys 27, 462-471 (2000).

${ }^{147} \mathrm{~L}$. Spies and T. Bortfeld, "Analytical scatter kernels for portal imaging at $6 \mathrm{MV}$," Med Phys 28, 553-559 (2001).

${ }^{148}$ L. Spies, M. Partridge, B.A. Groh, and T. Bortfeld, "An iterative algorithm for reconstructing incident beam distributions from transmission measurements using electronic portal imaging," Phys Med Biol 46, N203-211 (2001).

${ }^{149}$ W. Swindell and P.M. Evans, "Scattered radiation in portal images: a Monte Carlo simulation and a simple physical model," Med Phys 23, 63-73 (1996).

${ }^{150}$ V.N. Hansen, W. Swindell, and P.M. Evans, "Extraction of primary signal from EPIDs using only forward convolution," Med Phys 24, 1477-1484 (1997).

${ }^{151}$ M. Partridge and P.M. Evans, "The practical implementation of a scatter model for portal imaging at $10 \mathrm{MV}$," Phys Med Biol 43, 2685-2693 (1998).

${ }^{152}$ T.R. McNutt, T.R. Mackie, P. Reckwerdt, N. Papanikolaou, and B.R. Paliwal, "Calculation of portal dose using the convolution/superposition method," Med Phys 23, 527-535 (1996).

${ }^{153} \mathrm{M}$. Mohammadi, E. Bezak, and P. Reich, "The use of extended dose range film for dosimetric calibration of a scanning liquid-filled ionization chamber electronic portal imaging device," J Appl Clin Med Phys 8, 69-84 (2007).

${ }^{154}$ M. Mohammadi, E. Bezak, and P. Reich, "Comparison of two-dimensional transmitted dose maps: evaluation of existing algorithms," Australas Phys Eng Sci Med 29, 179-187 (2006).

${ }^{155}$ J.M. Kapatoes, G.H. Olivera, P.J. Reckwerdt, E.E. Fitchard, E.A. Schloesser, and T.R. Mackie, "Delivery verification in sequential and helical tomotherapy," Phys Med Biol 44, 1815-1841 (1999). 
${ }^{156}$ M.C. Kirby and P.C. Williams, "The use of an electronic portal imaging device for exit dosimetry and quality control measurements," Int J Radiat Oncol Biol Phys 31, 593-603 (1995).

${ }^{157}$ M.C. Kirby and P.C. Williams, "Measurement possibilities using an electronic portal imaging device," Radiother Oncol 29, 237-243 (1993).

${ }^{158}$ R. Boellaard, M. van Herk, H. Uiterwaal, and B. Mijnheer, "Two-dimensional exit dosimetry using a liquid-filled electronic portal imaging device and a convolution model," Radiother Oncol 44, 149-157 (1997).

${ }^{159}$ R. Boellaard, M. Essers, M. van Herk, and B.J. Mijnheer, "New method to obtain the midplane dose using portal in vivo dosimetry," Int J Radiat Oncol Biol Phys 41, 465-474 (1998).

${ }^{160}$ R. Boellaard, M. van Herk, H. Uiterwaal, and B. Mijnheer, "First clinical tests using a liquidfilled electronic portal imaging device and a convolution model for the verification of the midplane dose," Radiother Oncol 47, 303-312 (1998).

${ }^{161}$ L.N. McDermott, M. Wendling, B. van Asselen, J. Stroom, J.J. Sonke, M. van Herk, and B.J. Mijnheer, "Clinical experience with EPID dosimetry for prostate IMRT pre-treatment dose verification," Med Phys 33, 3921-3930 (2006).

${ }^{162}$ L.N. McDermott, M. Wendling, J.J. Sonke, M. van Herk, and B.J. Mijnheer, "Replacing pretreatment verification with in vivo EPID dosimetry for prostate IMRT," Int J Radiat Oncol Biol Phys 67, 1568-1577 (2007).

${ }^{163} \mathrm{G}$. Jarry and F. Verhaegen, "Patient-specific dosimetry of conventional and intensity modulated radiation therapy using a novel full Monte Carlo phase space reconstruction method from electronic portal images," Phys Med Biol 52, 2277-2299 (2007).

${ }^{164}$ M. Partridge, M. Ebert, and B.M. Hesse, "IMRT verification by three-dimensional dose reconstruction from portal beam measurements," Med Phys 29, 1847-1858 (2002).

${ }^{165}$ J. Chen, O. Morin, M. Aubin, M.K. Bucci, C.F. Chuang, and J. Pouliot, "Dose-guided radiation therapy with megavoltage cone-beam CT," Br J Radiol 79 Spec No 1, S87-98 (2006).

${ }^{166}$ R.J. Louwe, E.M. Damen, M. van Herk, A.W. Minken, O. Torzsok, and B.J. Mijnheer, "Threedimensional dose reconstruction of breast cancer treatment using portal imaging," Med Phys 30, 2376-2389 (2003).

${ }^{167}$ L.N. McDermott, M. Wendling, J. Nijkamp, A. Mans, J.J. Sonke, B.J. Mijnheer, and M. van Herk, "3D in vivo dose verification of entire hypo-fractionated IMRT treatments using an EPID and cone-beam CT," Radiother Oncol 86, 35-42 (2008).

${ }^{168}$ J.M. Kapatoes, G.H. Olivera, K.J. Ruchala, J.B. Smilowitz, P.J. Reckwerdt, and T.R. Mackie, "A feasible method for clinical delivery verification and dose reconstruction in tomotherapy," Med Phys 28, 528-542 (2001).

${ }^{169}$ J.M. Kapatoes, G.H. Olivera, J.P. Balog, H. Keller, P.J. Reckwerdt, and T.R. Mackie, "On the accuracy and effectiveness of dose reconstruction for tomotherapy," Phys Med Biol 46, 943-966 (2001).

${ }^{170}$ D.A. Low, W.B. Harms, S. Mutic, and J.A. Purdy, "A technique for the quantitative evaluation of dose distributions," Med Phys 25, 656-661 (1998).

${ }^{171}$ D.A. Low and J.F. Dempsey, "Evaluation of the gamma dose distribution comparison method," Med Phys 30, 2455-2464 (2003).

${ }^{172}$ A. Bakai, M. Alber, and F. Nusslin, "A revision of the gamma-evaluation concept for the comparison of dose distributions," Phys Med Biol 48, 3543-3553 (2003).

${ }^{173}$ S.B. Jiang, G.C. Sharp, T. Neicu, R.I. Berbeco, S. Flampouri, and T. Bortfeld, "On dose distribution comparison," Phys Med Biol 51, 759-776 (2006).

${ }^{174}$ N.L. Childress, C. Bloch, R.A. White, M. Salehpour, and Rosen, II, "Detection of IMRT delivery errors using a quantitative 2D dosimetric verification system," Med Phys 32, 153-162 (2005).

${ }^{175}$ G.J. Budgell, B.A. Perrin, J.H. Mott, J. Fairfoul, and R.I. Mackay, "Quantitative analysis of patient-specific dosimetric IMRT verification," Phys Med Biol 50, 103-119 (2005). 
${ }^{176}$ S. Gillis, C. De Wagter, J. Bohsung, B. Perrin, P. Williams, and B.J. Mijnheer, "An inter-centre quality assurance network for IMRT verification: results of the ESTRO QUASIMODO project," Radiother Oncol 76, 340-353 (2005).

${ }^{177}$ M. Stock, B. Kroupa, and D. Georg, "Interpretation and evaluation of the gamma index and the gamma index angle for the verification of IMRT hybrid plans," Phys Med Biol 50, 399-411 (2005).

${ }^{178}$ E. Spezi and D.G. Lewis, "Gamma histograms for radiotherapy plan evaluation," Radiother Oncol 79, 224-230 (2006).

${ }^{179}$ M. van Zijtveld, M.L. Dirkx, H.C. de Boer, and B.J. Heijmen, "Dosimetric pre-treatment verification of IMRT using an EPID; clinical experience," Radiother Oncol 81, 168-175 (2006).

${ }^{180}$ M. Wendling, L.J. Zijp, L.N. McDermott, E.J. Smit, J.J. Sonke, B.J. Mijnheer, and M. van Herk, "A fast algorithm for gamma evaluation in 3D," Med Phys 34, 1647-1654 (2007).

${ }^{181}$ K.L. Pasma, M.L. Dirkx, M. Kroonwijk, A.G. Visser, and B.J. Heijmen, "Dosimetric verification of intensity modulated beams produced with dynamic multileaf collimation using an electronic portal imaging device," Med Phys 26, 2373-2378 (1999).

${ }^{182}$ P.M. Evans, E.M. Donovan, M. Partridge, P.J. Childs, D.J. Convery, S. Eagle, V.N. Hansen, B.L. Suter, and J.R. Yarnold, "The delivery of intensity modulated radiotherapy to the breast using multiple static fields," Radiother Oncol 57, 79-89 (2000).

${ }^{183}$ E. Donovan, N. Bleakley, E. Denholm, P. Evans, L. Gothard, J. Hanson, C. Peckitt, S. Reise, G. Ross, G. Sharp, R. Symonds-Tayler, D. Tait, and J. Yarnold, "Randomised trial of standard 2D radiotherapy (RT) versus intensity modulated radiotherapy (IMRT) in patients prescribed breast radiotherapy," Radiother Oncol 82, 254-264 (2007). 
PRE-TREATMENT DOSE VERIFICATION 



\section{CHAPTER}

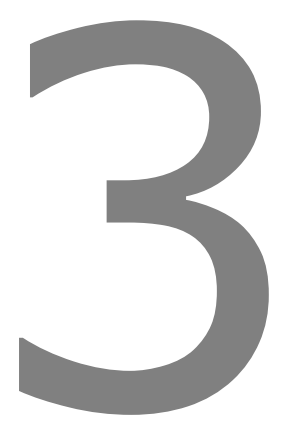

A Monte Carlo based three-dimensional dose reconstruction method derived from portal dose images

Wouter van Elmpt, Sebastiaan Nijsten, Robert Schiffeleers, André Dekker, Ben Mijnheer, Philippe Lambin and André Minken

Published in: Medical Physics 33(7):2426-2434, 2006. 


\begin{abstract}
Verification of intensity-modulated radiation therapy (IMRT) is necessary for adequate quality control of the treatment. Pre-treatment verification may trace possible differences between the planned dose and actual dose delivered to the patient. To estimate the impact of differences between planned and delivered photon beams, a threedimensional (3D) dose verification method has been developed that reconstructs the dose inside a phantom. The pre-treatment procedure is based on portal dose images measured with an electronic portal imaging device (EPID) of the separate beams, without the phantom in the beam and a 3D dose calculation engine based on Monte Carlo calculation. Measured grayscale portal images are converted into portal dose images. From these images the lateral scattered dose in the EPID is subtracted and the image is converted into energy fluence. Subsequently a phase-space distribution is sampled from the energy fluence and a 3D dose calculation in a phantom is started based on a Monte Carlo dose engine. The reconstruction model is compared to film and ionization chamber measurements for various field sizes. The reconstruction algorithm is also tested for an IMRT plan using $10 \mathrm{MV}$ photons delivered to a phantom and measured using films at several depths in the phantom. Depth dose curves for both 6 and $10 \mathrm{MV}$ photons are reconstructed with a maximum error generally smaller than $1 \%$ at depths larger than the build-up region, and smaller than $2 \%$ for the off-axis profiles, excluding the penumbra region. Absolute dose values are reconstructed within $1.5 \%$ for square field sizes ranging from 5 to $20 \mathrm{~cm}$ width. For the IMRT plan, the dose was reconstructed and compared to the dose distribution with film using the gamma evaluation, with a $3 \%$ and $3 \mathrm{~mm}$ criterion. $99 \%$ of the pixels inside the irradiated field had a gamma value smaller than one. The absolute dose at the isocenter agreed within $1 \%$ with the dose measured with an ionization chamber. It can be concluded that our new dose reconstruction algorithm is able to reconstruct the 3D dose distribution in phantoms with a high accuracy. This result is obtained by combining portal dose images measured prior to treatment with an accurate dose calculation engine.
\end{abstract}




\section{INTRODUCTION}

Intensity-modulated radiotherapy (IMRT) allows the delivery of radiation dose conformally to the tumor and/or sparing of normal tissues. IMRT can result in steep dose gradients between target volumes and organs at risk and therefore good quality control procedures are necessary of the total process of treatment planning, patient setup and beam delivery. Quality control procedures must be implemented before the start of the treatment, i.e. pre-treatment verification should be performed. ${ }^{1-6}$ Various verification techniques and a large number of homogeneous and inhomogeneous phantoms are available for IMRT verification. Also a variety of detectors are used including film, arrays and matrices of diodes and ionization chambers. Verification procedures can be divided according to the number of points that are checked in the phantom, and can be performed at a single point, in two-dimensions (2D), in multiple 2D planes, i.e. semi three-dimensional (3D), and using a full 3D reconstruction. All methods have their own advantages and disadvantages.

Point detectors, e.g. ionization chambers, in a phantom can be used for checking the dose delivered at a single point. This verification technique is fast and simple and can be used for an absolute dose verification of both the total plan and a beam-bybeam verification. The difficulty with point detectors is that differences are difficult to interpret in terms of tumor coverage or dose in normal tissue because only a single point is verified.

An extension of the single point dose measurement is a 2D plane measurement. Films, energy fluence detectors or electronic portal imaging devices (EPIDs) are suitable for measuring a 2D dose distribution in front of, inside, or behind a phantom. ${ }^{7-11}$ Films are widely used for checking the $2 \mathrm{D}$ dose distribution inside a phantom. This measurement technique is accurate but labor intensive. The total plan can be verified and beam-by-beam measurements are also possible. 2D energy fluence detectors that can be placed on top of the treatment couch or attached to the accelerator head can decrease the workload compared to film measurements. However, these measurement devices have the disadvantage that only a verification of the energy fluence of a single beam can be performed. With energy fluence detectors, or for beam-by-beam verification in general, it is not clear how possible differences add up in the total dose delivered.

EPIDs can be used for geometric verification of the patient position, of the multileaf collimator (MLC) setting or of the leaf trajectory control, but also for dosimetry purposes. If the EPID, which is attached permanently to the linear accelerator, is used for dosimetry this would decrease the workload compared to placing conventional dosimeters, energy fluence detectors or films.

There are several possibilities to use EPIDs for pre-treatment verification. The forward approach ${ }^{1,2,6,12-14}$ predicts the dose or the measured grayscale value at the portal imager based on the planned beams. This predicted dose is then compared with the measured dose and differences are quantified at the plane of the portal imager. The difficulty with this method is that it is not clear how differences at the plane of the EPID are related to the dose in the target volume or in normal tissues. A backward approach $^{3-5,15-20}$ is used to relate the measured portal dose to the dose at a point, 
plane or 3D volume inside the patient. These reconstructed dose data are then compared with the corresponding planned dose values.

Verifying the entrance energy fluence or the dose inside a phantom with ionization chamber, film or specially developed equipment is labor intensive. For the beam-bybeam analysis techniques it is not clear what the impact is of possible differences on the total 3D dose distribution. With simultaneous film measurements it is possible to measure in multiple planes of the phantom and to reconstruct a semi-3D dose distribution. This procedure is cumbersome for routine pre-treatment verification due to the high workload for developing, digitizing and comparing the films with the planned dose. Full 3D dose distribution verification will gain more insight in possible delivery problems. Several approaches are presented in the literature. Gels ${ }^{21}$ have the possibility for measuring a 3D dose distribution inside a phantom, but such a technique is not yet available for routine patient specific quality control and can only be used to verify class solution treatments. In an approach to pre-treatment 3D dose verification, Steciw et al. $^{5}$ implemented a dose verification procedure based on the measured energy fluence, and then applied the same treatment planning system as used for the original planning to calculate the dose inside the patient and to estimate the impact of possible differences. Errors in the dose calculation engine of the planning system are, however, not detected in these approaches. Renner et al. ${ }^{4}$ used film dosimetry and a dose calculation algorithm based on a convolution/superposition algorithm to reconstruct the $3 \mathrm{D}$ delivered dose in the patient. An independent pre-treatment verification is necessary to detect all possible types of errors, e.g. incorrect treatment parameter transfer, dose calculation errors due to limitations of the algorithm itself and its implementation in the treatment planning system, uncertainties in the leaf position, and possible delivery errors of the linear accelerator.

To overcome the limitations of the current pre-treatment verification models, that can only verify the dose at a single point or in a plane, and the limitation of entrance energy fluence detectors that can only measure a single beam with no information on how differences may add up or cancel out in the 3D dose distribution, a 3D dose reconstruction model is necessary.

The aim of our work is to develop a method that can reconstruct the 3D dose distribution actually delivered to a phantom or patient, based on a measurement of portal dose images of the separate beams before the start of treatment. Our 3D pretreatment dose verification should allow an independent verification of IMRT delivery by using measured portal images in combination with an independent dose calculation algorithm based on a Monte Carlo dose engine. Such a model would replace the need for performing film or ionization chamber measurements in a phantom for pretreatment quality control procedures of (IMRT) treatments. The result of the independent $3 D$ dose calculation must then be compared with the planned dose distribution. In the present study, this method is presented and verified for homogeneous phantoms. At a later stage the model will be extended to situations having inhomogeneous (tissue) densities. 


\section{MATERIALS AND METHODS}

\section{D dose reconstruction model}

The pre-treatment dose reconstruction model is based on measuring portal images without the phantom in the beam and an independent dose calculation algorithm. The reconstruction model consists of 4 steps. First, a portal image is converted to a portal dose image. Second, from this portal dose image the energy fluence exiting the linac is extracted. The energy fluence is defined as the total energy of the photons passing through an area. The third step is to sample a phase space distribution from the energy fluence. And finally, a dose calculation based on a Monte Carlo calculation in a phantom is performed. These 4 steps will be discussed in the next sections in more detail.

\section{Portal dose measurement}

A portal image is measured under the same conditions as the actual treatment but without an object placed in the beam. This portal image is converted into a portal dose image (PDI) using an in-house developed calibration model, similar to the procedure described by Chen et al. ${ }^{7}$ The calibration model converts the EPID image to a PDI with an error smaller than 1\% (SD) for images without an object in the beam. In our study this PDI represents the 2D dose distribution measured at a depth of $5 \mathrm{~cm}$ under full scatter conditions in a water tank, with a source-to-surface distance of $145 \mathrm{~cm}$, resulting in an effective source-to-detector distance of $150 \mathrm{~cm}$.

\section{Energy fluence extraction}

The primary dose is extracted from the measured PDI by deconvolving the portal dose with a lateral scatter kernel. The kernel is calculated from dose measurements with a mini-phantom and dose measurements under full scatter conditions. The portal dose $D$ can be described by a primary and a lateral scatter component $D^{P}$ and $D^{S}$, respectively:

$$
D=D^{P}+D^{S}
$$

The lateral scattered dose $D^{S}$ is described by a convolution of the primary dose with a lateral scatter kernel $\mathrm{K}$,

$$
D^{S}=D^{P} \otimes K
$$

This kernel can be fitted from on-axis phantom scatter measurements at the position of the EPID. The phantom scatter correction factor $S_{p}$ is determined by using the collimator and total scatter correction factor $S_{c}$ and $S_{c, p}$, respectively, measured at a source-to-detector distance of $150 \mathrm{~cm}$, normalized to a $10 \times 10 \mathrm{~cm}^{2}$ field. The lateral scatter kernel is determined from the following equation:

$$
\frac{S_{c, p}}{S_{c}}=S_{p}=c_{7}\left(1+\iint f(r) \cdot K(r) d^{2} r\right)
$$


with

$$
K(r)=c_{1} \exp \left(-c_{2} r\right)+c_{3} \exp \left(-c_{4} r\right)+c_{5} \exp \left(-c_{6} r\right),
$$

and $c_{7}$ is a parameter describing the relation between the primary dose and the dose measured under full scatter conditions of a $10 \times 10 \mathrm{~cm}^{2}$ field, $f(r)$ is the primary dose profile $D^{P}(r)$ normalized to the central axis dose $(r=0)$ for the measured field,

$$
c_{7}=\frac{D^{P}{ }_{10 \times 10}(r=0)}{D_{10 \times 10}(r=0)}, f(r)=\frac{D^{P}(r)}{D^{P}(r=0)} .
$$

For a derivation of Eq. (3.3) see the Appendix.

The energy fluence $\Psi$ is assumed to be proportional to the primary dose $D^{P}$

$$
\Psi=\frac{D^{P}}{\frac{\mu}{\rho} \exp (-\mu t)},
$$

with $\rho$ the density of the medium and the depth of the measurement point. The linear attenuation coefficient $\mu(\theta)$ at an off-axis angle $\theta$ is assumed to be described by the on-axis attenuation coefficient $\mu(\theta=0)$ multiplied by an off-axis correction factor described by Tailor et al, $^{22}$

$$
\frac{\mu(\theta)}{\mu(\theta=0)}=1+0.00181 \theta+0.00202 \theta^{2}-0.0000942 \theta^{3} \text {. }
$$

\section{Phase space reconstruction}

The starting point of our dose calculation, based on the Monte Carlo code $\mathrm{XVMC}^{23}$, is a phase space distribution (PSD). This PSD is reconstructed using a simple point source model that is located at the target of the linac. ${ }^{24,25}$ From the energy fluence distribution and a pre-defined spectrum, a random position and energy are sampled, respectively. Such a reconstructed PSD has 8 parameters: three parameters indicating the position, three parameters describing the direction and two parameters describing the energy and the statistical weight of the photon.

The direction and position of the individual photons is calculated by assuming that the photons originate from a point source located at the target. This position can be sampled from a point in the reconstructed energy fluence distribution. Six parameters indicating the position and direction of the photon below the collimator jaws, MLC and blocks, are calculated.

The spectrum parameters are taken from the commissioning procedure of the XVMC code. ${ }^{26}$ Briefly, the energy of the photon $E$ is sampled from an on-axis spectrum and multiplied with a correction for the off-axis position to incorporate beam softening. The on-axis spectrum is derived from measured and simulated percentage depth dose curves and fitted to an analytical function. ${ }^{26}$ This on-axis energy spectrum $p(E)$ is described by a function characterized by 4 parameters: $I, b, E_{\min }$ and $E_{\max }$, 


$$
p(E) d E=N E^{l} \exp (-b E) d E,
$$

with $E_{\min }<E<E_{\max }$, and $\mathrm{N}$ a normalization factor, chosen in such a way that the sum of all chances is unity. The energy of the off-axis photons are sampled from the on-axis spectrum and an off-axis correction $s(\theta)$ is applied, ${ }^{22,26}$

$$
E(\theta)=s(\theta) E
$$

with $s(\theta)$ the off-axis correction for angle $\theta$,

$$
s(\theta)=\left(\frac{\mu(\theta=0)}{\mu(\theta)}\right)^{2.22} .
$$

The statistical weight of the photon is sampled from the measured energy fluence distribution. To incorporate the absolute value of the energy fluence at a specific point in the reconstructed phase space distribution, the energy fluence is divided by the offaxis correction factor so that the total energy fluence at an off-axis position is kept constant and equal to the energy fluence extracted from the portal dose image at that position.

\section{Monte Carlo calculation}

The reconstructed phase space distribution is the starting point for an independent dose calculation based on Monte Carlo calculation. The Monte Carlo code $\mathrm{XVMC}^{23}$ is used for a fast calculation of the 3D dose inside the phantom. Briefly, this code was derived from the Voxel Monte Carlo ${ }^{27}$ code for electron dose calculations and several improvements such as variance reduction techniques ${ }^{28}$ are used for a fast and accurate dose calculation. For a detailed explanation of the code see Fippel et al. ${ }^{23}$

\section{Linear accelerator and electronic portal imaging device}

The Electronic Portal Imaging Device (EPID) used for the measurements is a Siemens OptiVue 1000 amorphous silicon type EPID mounted on a Siemens Oncor Avant Garde linear accelerator (Siemens Medical Solutions, Concord, USA) equipped with 6 and 10 MV photons. The EPID is calibrated using an in-house developed model to measure a 2D dose distribution in a water tank at $5 \mathrm{~cm}$ depth placed at $145 \mathrm{~cm}$ from the focus of the linac. For the work presented here the distance of the EPIDs is fixed at a source-toimager distance of $150 \mathrm{~cm}$, with an effective area of $41 \times 41 \mathrm{~cm}^{2}$, the image size is resampled to $512 \times 512$ pixels with a pixel spacing of $0.8 \mathrm{~mm}$. An additional copper plate of $3 \mathrm{~mm}$ is mounted on top of the EPID to reduce the over-response due to lowenergy scattered photons that are incident on the sensitive layer of the EPID. ${ }^{8}$

\section{Phantom study}

The 3D dose reconstruction method has been tested using homogeneous phantoms. First, the model parameters of the reconstruction algorithm are derived. Second, the method is compared to measurements in a water phantom with an ionization cham- 
ber. An irregularly shaped MLC segment is also measured with film in a homogeneous phantom. Third, an actual IMRT plan is delivered to a homogeneous phantom with various film inserts and an insert for an ionization chamber.

\section{Model parameter derivation}

The parameters of the model, i.e. the coefficients of the lateral scatter kernel, the energy fluence and spectrum parameters, are derived from measurements. For the determination of the lateral scatter kernel square fields of widths $3,6,10,15,20,25$, 30 and $35 \mathrm{~cm}$ were used, measured both under full scatter conditions and with a miniphantom. The source-to-detector distance is $150 \mathrm{~cm}$ and 100 monitor units for both 6 and $10 \mathrm{MV}$ photons were given. The mini-phantom is a polystyrene cylindrical phantom with a diameter of $3 \mathrm{~cm}$ and the effective point of measurement is located at $5 \mathrm{~cm}$ water equivalent below the surface of the detector (CC13, Scanditronix Wellhofer, Schwarzenbruck, Germany) read out by an electrometer (Model 35040, Keithley Instruments Inc., Cleveland, USA) and positioned at a source-to-detector distance of $150 \mathrm{~cm}$, equal to the point of measurement of the dose under full scatter conditions. A diagonal profile of the largest field is also measured with the mini-phantom to yield an estimate of $f(r)$ in Eq. (3.3), while the boundary values are chosen according to the field width. The shape of the profile inside the field is assumed to be independent of the field size. The lateral scatter kernel $K$ is fitted to Eq. (3.4). The coefficients $c_{i}$ are determined by a fitting procedure using an unconstrained non-linear minimization problem implemented in Matlab (Matlab 7.0.4, The Mathworks, Natick, USA). The primary dose is calculated from the measured dose under full scatter conditions (Eq. 3.1) in an

Table 3.1: Overview of the parameter values of the reconstruction model for 6 and $10 \mathrm{MV}$ photons.

\begin{tabular}{|c|c|c|}
\hline Fit parameter & $6 \mathrm{MV}$ & $10 \mathrm{MV}$ \\
\hline$c_{1}\left[10^{-3}\right]$ & $6.6350^{*}$ & $11.321^{*}$ \\
\hline$c_{2}\left[10^{-1} \mathrm{~cm}^{-1}\right]$ & $8.8738^{*}$ & $9.3365^{*}$ \\
\hline$c_{3}\left[10^{-4}\right]$ & $13.723^{*}$ & $2.4679^{*}$ \\
\hline $\mathrm{c}_{4}\left[10^{-1} \mathrm{~cm}^{-1}\right]$ & $3.0911^{*}$ & $1.7922^{*}$ \\
\hline$C_{5}\left[10^{-4}\right]$ & $1.0679^{*}$ & $1.1905^{*}$ \\
\hline$c_{6}\left[10^{-1} \mathrm{~cm}^{-1}\right]$ & $0.9468^{*}$ & $1.4860^{*}$ \\
\hline$c_{7}[-]$ & $0.8825^{*}$ & $0.8952^{*}$ \\
\hline$\mu(\theta=0)\left[10^{-2} \mathrm{~cm}^{-1}\right]$ & $4.68 \pm 0.05$ & $3.57 \pm 0.05$ \\
\hline$\rho\left[\mathrm{g} / \mathrm{cm}^{3}\right]$ & 1.000 & 1.000 \\
\hline I [-] & $0.1320^{* *}$ & $0.8552^{* *}$ \\
\hline $\mathrm{b}\left[\mathrm{MeV}^{-1}\right]$ & $0.6478^{* *}$ & $0.5552^{* *}$ \\
\hline $\mathrm{E}_{\min }[\mathrm{MeV}]$ & $0.25^{* *}$ & $0.25^{* *}$ \\
\hline $\mathrm{E}_{\max }[\mathrm{MeV}]$ & $7.00^{* *}$ & $11.00^{* *}$ \\
\hline \multicolumn{3}{|c|}{$\begin{array}{l}\text { * The uncertainty in the parameters is indicated by the actual fitting procedure of the phan- } \\
\text { tom scatter correction factor. The model described this factor with a maximum error of } 0.2 \% \\
\text { between measured and fitted } \mathrm{S}_{\mathrm{p}} \text { values. } \\
\text { The parameters are taken from the commissioning procedure of the XVMC model and are } \\
\text { fitted from a measured percentage depth dose curve. The maximum difference between the } \\
\text { reconstructed curve and the measured curve was } 1 \% \text {. }\end{array}$} \\
\hline
\end{tabular}


iterative way using convolution and deconvolution, programmed using Fast Fourier Transforms for speed considerations.

The spectrum parameters $I, b, E_{\min }$ and $E_{\max }$ are taken from the commissioning procedure of the XVMC code using the Virtual Energy Fluence model. ${ }^{26}$ The fitted energy parameters can be found in Table 3.1.

The on-axis attenuation coefficient $\mu(\theta=0)$ is determined by transmission measurements of a small field $(3 \mathrm{~cm} \times 3 \mathrm{~cm})$ through a $10 \mathrm{~cm}$ thick polystyrene phantom.

The absolute dose calibration constant is determined by reconstructing the dose at a single point, i.e. at a depth of $10 \mathrm{~cm}$ and an SSD $90 \mathrm{~cm}$, for a $10 \mathrm{~cm} \times 10 \mathrm{~cm}$ square field and relating the value to the absolute dose value measured at the same point in a water phantom.

\section{Model verification measurements}

To test the accuracy of the reconstruction model various symmetric field sizes are used with widths of 5,10,15, and $20 \mathrm{~cm}$ for both 6 and $10 \mathrm{MV}$ photons. The percentage depth dose curves and off-axis profiles at various depths (5, 10, 20 and $30 \mathrm{~cm}$ ) are measured with the CC13 ionization chamber in a water phantom (Blue Phantom, Scanditronix Wellhofer, Schwarzenbruck, Germany) with a source-to-surface distance of $90 \mathrm{~cm}$ and are compared to the reconstructed dose.

Phantom measurements are used for checking the dose reconstruction model of an irregularly shaped MLC field using a photon beam of $6 \mathrm{MV}$. A phantom with dimensions $30 \mathrm{~cm} \times 30 \mathrm{~cm} \times 21 \mathrm{~cm}$ consisting of square slabs of about $2 \mathrm{~cm}$ polystyrene thickness is used to position EDR2 films (Eastman Kodak Company, Rochester, NY, USA) at different depths. For this measurement two films are placed at 10.5 and $15.0 \mathrm{~cm}$ depth from the surface $(S S D 90.0 \mathrm{~cm}$ ) of the phantom. The films are processed with the Kodak X-Omat 1000 film processor and digitized using a VXR-16 scanner (Vidar Systems Corporation, Herndon, VA, USA).

\section{IMRT plan and measurements}

An IMRT plan, generated using our treatment planning system (XiO 4.2.2, CMS, St. Louis, MO, USA), intended for a prostate treatment using $10 \mathrm{MV}$ photons consisting of 5 beams (gantry angles of 36, 100, 180, 260 and 324 degrees) and 53 segments in total, is delivered to the phantom described before. Films are placed at 4.3, 8.6, 13.0 and $17.3 \mathrm{~cm}$ depth in the phantom. For checking the absolute dose, one polystyrene slab is replaced by another slab containing a hole, located at the isocenter, for the placement of a Farmer-type ionization chamber (NE 2505/3, NE Technology Ltd, Reading, UK).

\section{PDI measurements and reconstruction}

EPID measurements for the square fields and the MLC field have been performed without the phantom in the beam. For the IMRT plan, the various segments for a single beam are integrated in the EPID software (Coherence-Portal Imaging Workspace, Siemens) resulting in a single image per beam (gantry angle). The gantry angle for 
acquiring the EPID images is forced to 0 degrees, because for portal dose acquisition a copper plate of $3 \mathrm{~mm}$ has been positioned on top of the EPID. This plate was, at the time of measurement not permanently fixed at the EPID and had to be placed manually on top of the EPID in order to perform the portal dose measurements. For the 3D dose reconstruction, however, the gantry angle was changed to the planned gantry angle that was used for the film measurements.

The voxel size and calculation grid for the water phantom verification measurements is $0.50 \times 0.50 \times 0.50 \mathrm{~cm}^{3}$, whereas for the analysis of the film measurements a smaller grid of $0.25 \times 0.25 \times 0.25 \mathrm{~cm}^{3}$ is used. The number of simulated photons was adjusted until a statistical error smaller than $1 \%$ was achieved for all presented data.

\section{RESULTS}

\section{Model parameters}

The results of the on-axis phantom scatter measurements are shown in Figure 3.1. From these measurements and the profile along the diagonal of the largest field, the lateral scatter kernel was fitted. This kernel is derived for a depth of $5 \mathrm{~cm}$ and an SSD of $145 \mathrm{~cm}$ and is shown in Figure 3.1, while the parameters of the fit are also presented in Table 3.1. The error of the fit was for both the 6 and $10 \mathrm{MV}$ beam below $0.2 \%$ (maximum difference). The attenuation coefficient was chosen according to transmission measurements of a small field $(3 \mathrm{~cm} \times 3 \mathrm{~cm})$ using a $10 \mathrm{~cm}$ thick polystyrene phantom. The on-axis attenuation coefficients $\mu(\theta=0)$ are $4.68 \cdot 10^{-2} \mathrm{~cm}^{-1}$ and $3.57 \cdot 10^{-2} \mathrm{~cm}^{-1}$ for the 6 and $10 \mathrm{MV}$ beam, respectively.

\section{Model verification measurements}

Figures 3.2-3.4 show reconstructed and measured beam profiles and depth dose curves for 6 and $10 \mathrm{MV}$ photons. The agreement between ionization chamber measurements and reconstructed data is within a few percent. The profiles, shown in
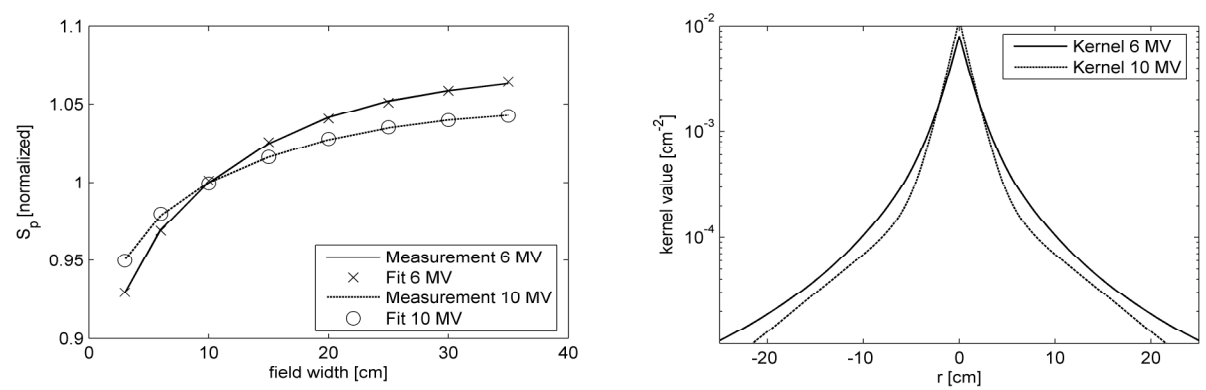

Figure 3.1: Measured and fitted phantom scatter correction factors (left) and the corresponding lateral scatter kernel (right), for the 6 and $10 \mathrm{MV}$ photon beams. 

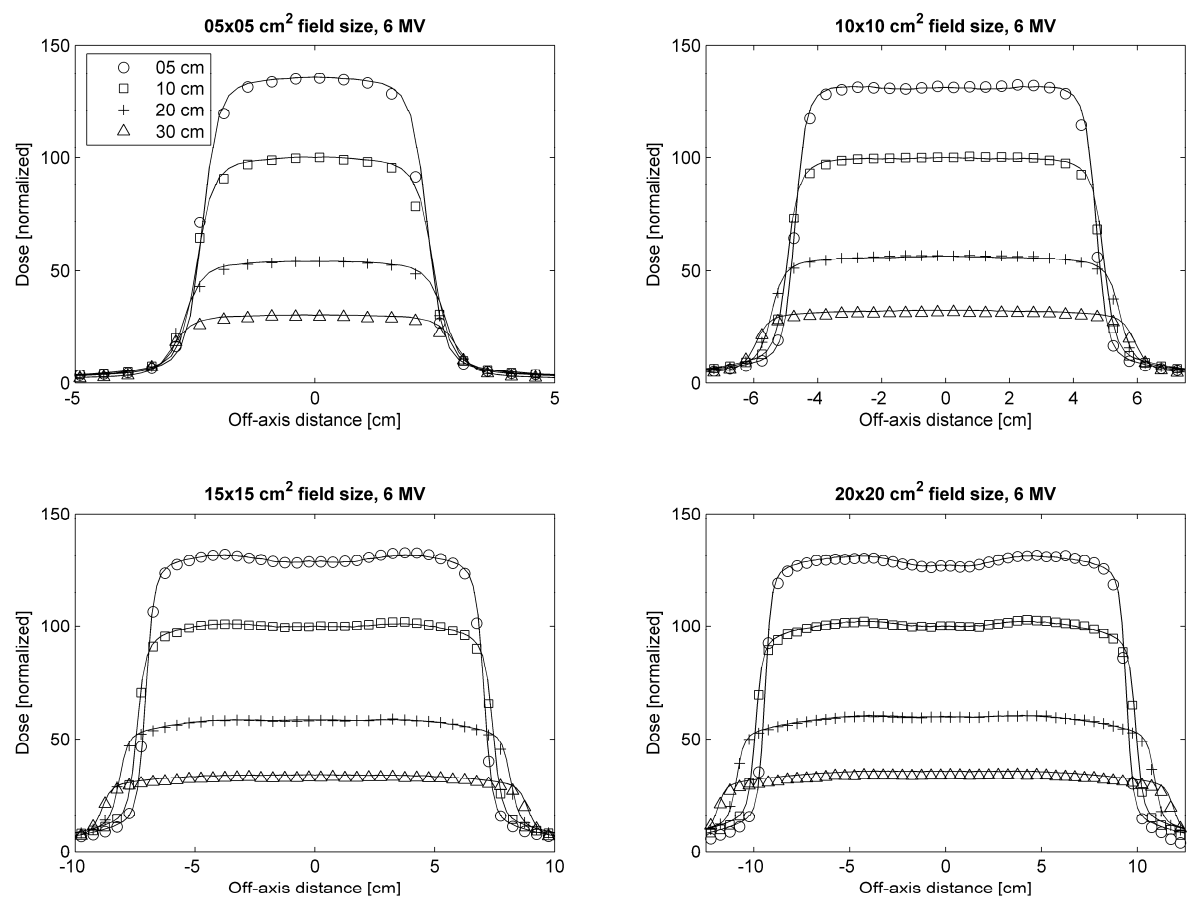

Figure 3.2: Measured and reconstructed beam profiles for various field sizes and various depths for a $6 \mathrm{MV}$ photon beam, normalized at the measured and reconstructed value on the central axis at a depth of $10 \mathrm{~cm}$.

Figures 3.2 and 3.3, are for all field sizes reproduced within $2 \%$ of the dose at the center of the profile, both inside and outside the field (excluding the penumbra) compared to ionization chamber measurements. Depth dose curves for all measured field sizes are reconstructed with an error smaller than 1\% (maximum difference) at depths larger than dose maximum $\left(D_{\max }\right)$. At shallow depths around $D_{\max }$ this difference is larger, which is shown in Figure 3.4. Absolute values of the reconstructed dose have been compared with measured output factors and are shown in Figure 3.5. For small fields, e.g. $5 \mathrm{~cm} \times 5 \mathrm{~cm}$, there is an overestimation of the reconstructed dose compared to the measured dose, while for the larger field sizes, e.g. $20 \mathrm{~cm} \times 20 \mathrm{~cm}$, the opposite effect occurs. This difference is however small, $<1.5 \%$ at maximum, for the 6 and $10 \mathrm{MV}$ beams.

The measured and reconstructed 2D dose distribution at $15.0 \mathrm{~cm}$ depth in the phantom of the $6 \mathrm{MV}$ MLC-shaped field is shown in Figure 3.6, normalized at the central axis. A gamma evaluation ${ }^{29,30}$, with $3 \%$ maximum dose difference and $3 \mathrm{~mm}$ distance-to-agreement criterion, is performed to quantify the differences between the measured and reconstructed dose distribution. The percentage of gamma-values, over a rectangular area defined by the outer leaf positions, smaller than unity is larger than $99 \%$ for both films at 10.5 and $15.0 \mathrm{~cm}$ depth in the phantom. 

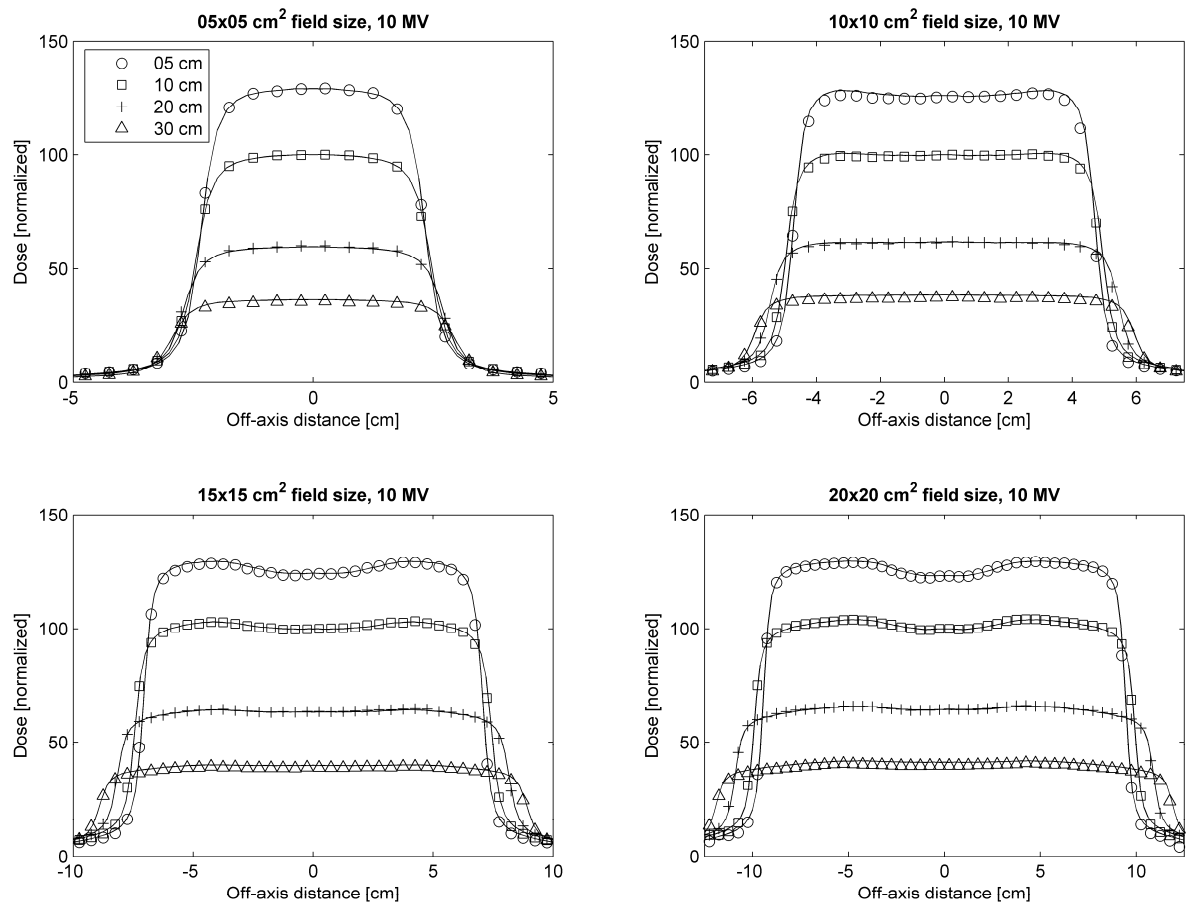

Figure 3.3: Measured and reconstructed beam profiles for various field sizes and various depths for a $10 \mathrm{MV}$ photon beam, normalized at the measured and reconstructed value on the central axis at a depth of $10 \mathrm{~cm}$.

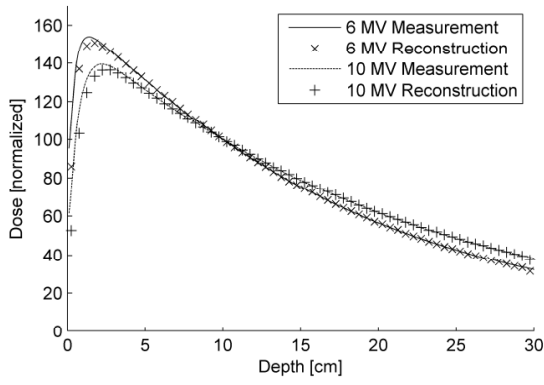

Figure 3.4: Measured and reconstructed percentage depth dose curves for a field size of $10 \mathrm{~cm} \times 10 \mathrm{~cm}, 6$ and $10 \mathrm{MV}$ photons, normalized at $10 \mathrm{~cm}$ depth.

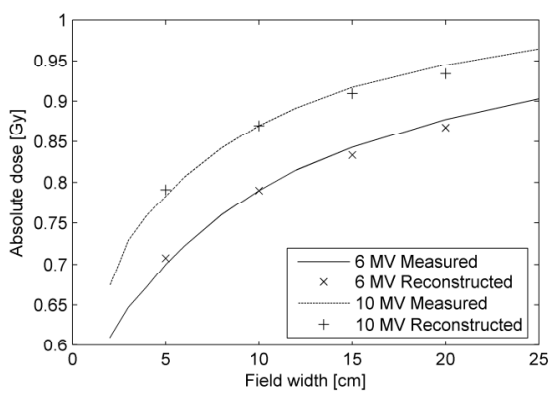

Figure 3.5: Measured and reconstructed absolute dose values at $10 \mathrm{~cm}$ depth in a water phantom (SSD $90 \mathrm{~cm}$ ) for 6 and 10 MV photons. For each measurement 100 monitor units were given. 

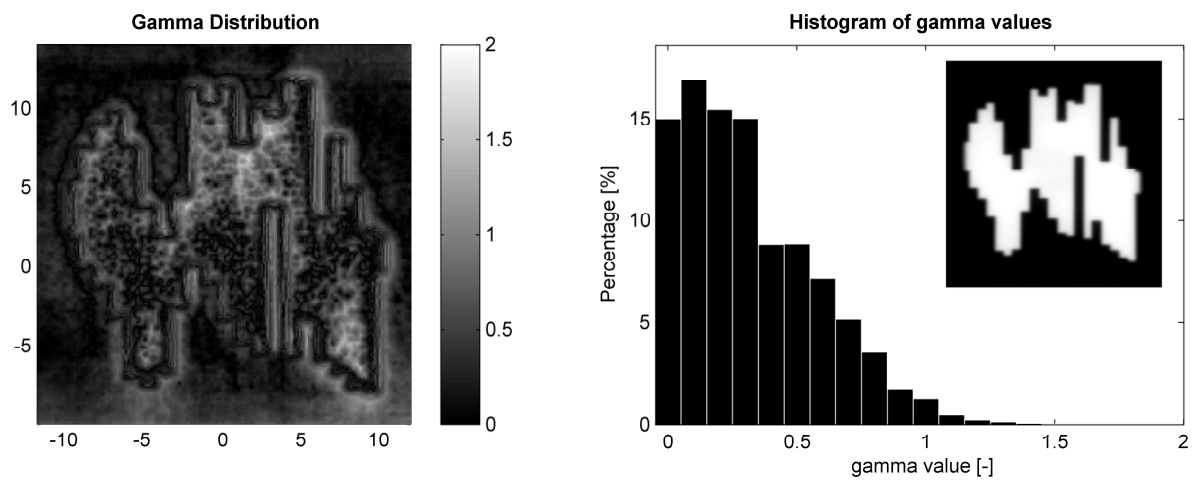

Figure 3.6: Gamma evaluation, with 3\% (of maximum) dose and $3 \mathrm{~mm}$ distance-to-agreement criteria, of a $6 \mathrm{MV}$ MLC-shaped segment and a histogram of the gamma-values (right). The measured portal dose image is shown in the right figure. The film measurement and dose reconstruction have been performed at $15.0 \mathrm{~cm}$ depth in the phantom.

\section{IMRT verification measurements}

The IMRT phantom plan has been checked by verifying the total dose distribution using film measurements in various planes. An ionization chamber measurement is used to verify the absolute dose at a single point in the phantom. The film measurements and reconstruction data are normalized on the central axis value. The gamma evaluation for the 10 MV IMRT plan is shown in Figure 3.7. A histogram of gamma values is calculated inside the irradiated field which is defined from $x=-5$ to $+5 \mathrm{~cm}$ and $y=-10$ to +10 $\mathrm{cm}$. Gamma values are generally below unity. The percentage of gamma-values $>1$ are $1.2 \%, 0.2 \%, 2.3 \%$ and $0.6 \%$ for the data at $4.3,8.6,13.0$ and $17.3 \mathrm{~cm}$ depth in the phantom. The somewhat higher number of gamma values $>1$ for the film placed at $13.0 \mathrm{~cm}$ depth was due to a difference between reconstructed and measured dose in a low dose area with a steep dose gradient towards the edge of the phantom. The dose difference between reconstructed and measured absolute dose was small, 1\%; the reconstructed dose was $2.13 \mathrm{~Gy}$ compared to $2.15 \mathrm{~Gy}$ for the dose measured with the ionization chamber.

\section{DISCUSSION}

Our new method reconstructs the 3D dose distribution in a homogeneous phantom based on the actual delivered beams measured with an EPID prior to treatment without the object placed in the beam. An accurate and easy to use reconstruction model has been developed that is able to reconstruct the 3D dose distribution in a homogeneous phantom independent of the treatment planning system, and based on the actual measured portal images.

The energy parameters are taken from the commissioning procedure of the XVMC code. The parameters can easily be derived from a measured depth dose curve. The 

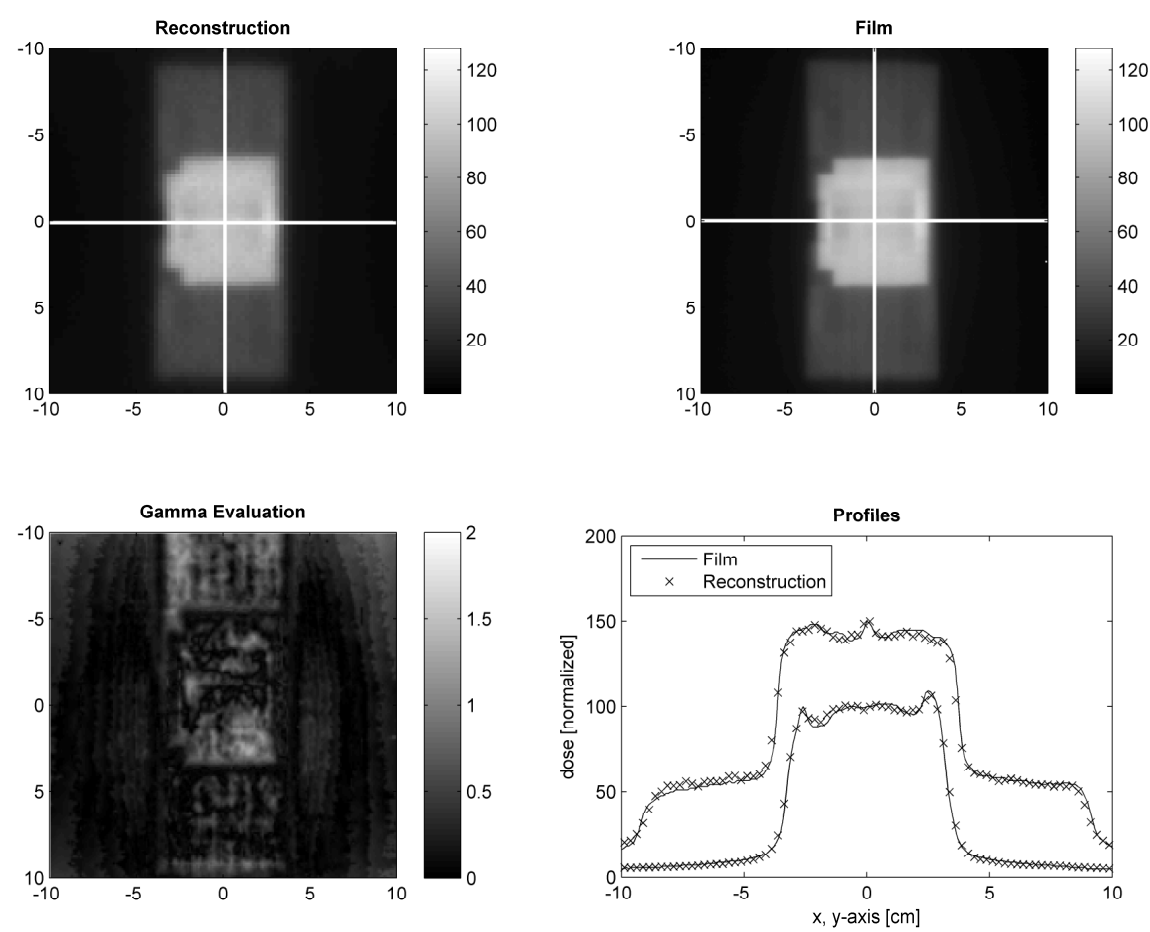

Figure 3.7: Reconstructed and measured dose distribution of a 10 MV IMRT prostate plan at 17.3 $\mathrm{cm}$ depth in the phantom are presented in the upper left and right plot, respectively. Differences between reconstructed dose and film, shown as a gamma evaluation, are given in the lower left plot. $3 \%$ of maximum dose and $3 \mathrm{~mm}$ distance-to-agreement criteria were used. In the lower right plot the reconstructed and film profiles for a horizontal and a vertical profile are shown. The vertical profiles are multiplied by a factor 1.5 in the lower right plot for a clear visual representation.

reconstructed percentage depth dose values are in good agreement with the measured data. There is, however, a small difference in the build-up region. This could be due to electron contamination, which is not modeled in the reconstruction model, but accurate measurements with the ionization chamber are also difficult to perform due to the lack of electron equilibrium. An extra source of this electron contamination could be incorporated in the model to yield accurate surface doses, but for dose verification at depths larger than $D_{\max }$ this is not necessary. Also head scatter is not taken into account very accurately. The measured energy fluence at the position of the EPID also contains head scatter fluence that is, due to the point source model, assumed to originate from the target as well.

The lateral scatter kernel is derived from the measured on-axis dose under full scatter conditions and primary dose. The primary dose is reconstructed accurately from the portal dose. The reconstructed off-axis primary dose profiles at the position of the portal imager are in good agreement (maximum difference $<2 \%$, not shown) with the measured primary dose profiles, excluding the penumbra. This method of 
verification with a mini-phantom is, however, limited to regions with a low dose gradient and cannot be used in regions with steep dose gradients because of the volume averaging effect due to the finite size, $3 \mathrm{~cm}$ diameter, of the mini-phantom. However, the penumbra after the reconstruction of the dose inside the phantom is in good agreement with measurements, indicating that the primary dose extraction from the portal dose image is also valid in regions with a high dose gradient.

The agreement between the measured and reconstructed absolute dose is good, but there is a trend to overestimate the dose for smaller field sizes and underestimate the dose for larger field sizes compared to the measurements. Fippel et al. ${ }^{26}$ also found this difference for the XVMC dose calculations using the commissioning procedure of the Virtual Energy Fluence model. The reason for this small difference is still unknown and needs further investigation. For IMRT treatments that consist of a large number of small fields this difference can be minimized by determining the absolute conversion factor for the reconstruction model at a smaller field size than the $10 \mathrm{~cm} \times 10 \mathrm{~cm}$ field that was used.

For the square fields, the reconstructed off-axis and depth dose data show excellent agreement with the measurements in the water phantom. Also the MLC-shaped segment shows a good correlation between measured and reconstructed dose values. At some points in the off-axis region around $(x, y)=(8,-7) \mathrm{cm}$ there is a slightly larger difference. These small differences between measurement and reconstruction might be the result of differences in the acquisition of the portal image or uncertainties in the film measurement, voxel size effects of the dose calculation, repositioning of the leaves between two different fractions, or variation in the output of the linac.

The dose calculation is performed using a Monte Carlo dose engine. The presented 3D dose reconstruction method is in principle not restricted to Monte Carlo based methods. Renner et al. ${ }^{4}$ used a similar approach by using film dosimetry and a dose calculation algorithm based on a convolution/superposition algorithm. Steciw et al. ${ }^{5}$ used the measured energy fluence and the treatment planning system's algorithm to estimate the impact of possible differences. Errors in the dose calculation engine of the planning system are, however, not detected in this approach.

The reconstruction algorithm is also not restricted to be used with homogeneous phantoms. Monte Carlo calculations are in principle superior in the dose calculation accuracy for inhomogeneous phantoms. Our reconstruction model must then also be validated with an inhomogeneous phantom study. The presented approach can then be used for dose reconstruction in the planning CT data of the patient. This opens possibilities for actual patient specific pre-treatment quality assurance procedures that can quantify the effects of differences between planned and delivered beams.

\section{CONCLUSION}

Our newly developed 3D dose verification model allows an accurate and independent method for the verification of complex (IMRT) treatments and the quantification of possible differences between the $3 \mathrm{D}$ dose distribution calculated with the treatment planning system and actual dose delivered. A set of measured portal images is the only 
input needed for the reconstruction model, which is the input for an accurate dose calculation based on a Monte Carlo dose engine.

\section{ACKNOWLEDGMENTS}

The authors would like to thank Dr. Matthias Fippel for the distribution of the XVMC code for dose calculations. Also gratitude is expressed to Chris Bouwman for helping with the fit of the energy spectra.

\section{APPENDIX: DERIVATION OF THE LATERAL SCATTER KERNEL}

The dose $D$ measured under full scatter conditions can be described as the summation of a primary dose $D^{P}$ and a lateral scattered dose $D^{S}$,

$$
D=D^{P}+D^{S}
$$

the lateral scattered dose is calculated using the primary dose and a lateral scatter kernel $\mathrm{K}$,

$$
D^{S}=D^{P} \otimes K .
$$

Evaluation of Eq. (3.A1) and (3.A2) at the central axis ( $r=0)$ and dividing this value by $D^{P}(r=0)$ leads to

$$
\frac{D(r=0)}{D^{P}(r=0)}=\frac{D^{P}(r=0)+\iint D^{P}\left(r^{\prime}\right) K\left(r^{\prime}\right) d^{2} r^{\prime}}{D^{P}(r=0)}=1+\iint f\left(r^{\prime}\right) K\left(r^{\prime}\right) d^{2} r^{\prime},
$$

with $f(r)$ the profile of the field, normalized to the central axis.

The collimator scatter correction factor $S_{c}$ and the total scatter correction factor $\mathrm{S}_{\mathrm{c}, \mathrm{p}}$ are defined as the measured primary dose and the measured dose under full scatter conditions, respectively, divided by the corresponding values for the reference field size, i.e. $10 \mathrm{~cm} \times 10 \mathrm{~cm}$,

$$
S_{c, p}=\frac{D}{D_{10 \times 10}}, S_{c}=\frac{D^{P}}{D^{P}{ }_{10 \times 10}} \text {. }
$$

Normalizing the measured primary and the dose measured under full scatter conditions and dividing the total scatter correction factor by the collimator scatter correction factor leads to the phantom scatter correction factor $S_{p}$,

$$
\frac{D(r=0) / D_{10 \times 10}(r=0)}{D^{P}(r=0) / D_{10 \times 10}^{P_{10}}(r=0)}=\frac{S_{c, p}}{S_{c}}=S_{p}=\frac{D_{10 \times 10}^{P}(r=0)}{D_{10 \times 10}(r=0)}\left(1+\iint f\left(r^{\prime}\right) K\left(r^{\prime}\right) d^{2} r^{\prime}\right) .
$$




\section{REFERENCES}

${ }^{1}$ A. Van Esch, T. Depuydt, and D. P. Huyskens, "The use of an aSi-based EPID for routine absolute dosimetric pre-treatment verification of dynamic IMRT fields," Radiother. Oncol. 71, 223234 (2004).

${ }^{2}$ A. Van Esch, B. Vanstraelen, J. Verstraete, G. Kutcher, and D. Huyskens, "Pre-treatment dosimetric verification by means of a liquid-filled electronic portal imaging device during dynamic delivery of intensity modulated treatment fields," Radiother. Oncol. 60, 181-190 (2001).

${ }^{3}$ M. Wendling, R. J. W. Louwe, L. N. McDermott, J. J. Sonke, M. van Herk, and B. J. Mijnheer, "Accurate two-dimensional IMRT verification using a back-projection EPID dosimetry method," Med. Phys. 33, 259-273 (2006).

${ }^{4}$ W. D. Renner, M. Sarfaraz, M. A. Earl, and C. X. Yu, "A dose delivery verification method for conventional and intensity-modulated radiation therapy using measured field fluence distributions," Med. Phys. 30, 2996-3005 (2003).

${ }^{5}$ S. Steciw, B. Warkentin, S. Rathee, and B. G. Fallone, "Three-dimensional IMRT verification with a flat-panel EPID," Med. Phys. 32, 600-612 (2005).

${ }^{6}$ S. M. J. J. G. Nijsten, A. W. H. Minken, P. Lambin, and I. A. D. Bruinvis, "Verification of treatment parameter transfer by means of electronic portal dosimetry," Med. Phys. 31, 341-347 (2004).

7J. Chen, C. F. Chuang, O. Morin, M. Aubin, and J. Pouliot, "Calibration of an amorphous-silicon flat panel portal imager for exit-beam dosimetry," Med. Phys. 33, 584-594 (2006).

${ }^{8}$ L. N. McDermott, R. J. W. Louwe, J. J. Sonke, M. B. van Herk, and B. J. Mijnheer, "Dose-response and ghosting effects of an amorphous silicon electronic portal imaging device," Med. Phys. 31, 285-295 (2004).

${ }^{9}$ B. J. M. Heijmen, K. L. Pasma, M. Kroonwijk, V. G. M. Althof, J. C. J. de Boer, A. G. Visser, and H. Huizenga, "Portal dose measurement in radiotherapy using an electronic portal imaging device (EPID)," Phys. Med. Biol. 40, 1943-1955 (1995).

${ }^{10}$ K. L. Pasma, M. Kroonwijk, J. C. J. de Boer, A. G. Visser, and B. J. M. Heijmen, "Accurate portal dose measurement with a fluoroscopic electronic portal imaging device (EPID) for open and wedged beams and dynamic multileaf collimation," Phys. Med. Biol. 43, 2047-2060 (1998).

${ }^{11}$ P. B. Greer, and C. C. Popescu, "Dosimetric properties of an amorphous silicon electronic portal imaging device for verification of dynamic intensity modulated radiation therapy," Med Phys 30, 1618-1627 (2003).

${ }^{12}$ K. L. Pasma, B. J. M. Heijmen, M. Kroonwijk, and A. G. Visser, "Portal dose image (PDI) prediction for dosimetric treatment verification in radiotherapy. I. An algorithm for open beams," Med. Phys. 25, 830-840 (1998).

${ }^{13}$ W. J. C. van Elmpt, S. M. J. J. G. Nijsten, B. J. Mijnheer, and A. W. H. Minken, "Experimental verification of a portal dose prediction model," Med. Phys. 32, 2805-2818 (2005).

${ }^{14}$ K. L. Pasma, S. C. Vieira, and B. J. M. Heijmen, "Portal dose image prediction for dosimetric treatment verification in radiotherapy. II. An algorithm for wedged beams," Med. Phys. 29, 925-931 (2002).

${ }^{15}$ K. L. Pasma, M. Kroonwijk, S. Quint, A. G. Visser, and B. J. M. Heijmen, "Transit dosimetry with an electronic portal imaging device (EPID) for 115 prostate cancer patients," Int. J. Radiat. Oncol. Biol. Phys. 45, 1297-1303 (1999).

${ }^{16}$ R. J. W. Louwe, E. M. F. Damen, M. van Herk, A. W. H. Minken, O. Torzsok, and B. J. Mijnheer, "Three-dimensional dose reconstruction of breast cancer treatment using portal imaging," Med. Phys. 30, 2376-2389 (2003).

${ }^{17}$ R. Boellaard, M. Essers, M. van Herk, and B. J. Mijnheer, "New method to obtain the midplane dose using portal in vivo dosimetry," Int. J. Radiat. Oncol. Biol. Phys. 41, 465-474 (1998). 
${ }^{18}$ R. Boellaard, M. van Herk, H. Uiterwaal, and B. Mijnheer, "First clinical tests using a liquid-filled electronic portal imaging device and a convolution model for the verification of the midplane dose," Radiother. Oncol. 47, 303-312 (1998).

${ }^{19}$ M. Partridge, M. Ebert, and B. M. Hesse, "IMRT verification by three-dimensional dose reconstruction from portal beam measurements," Med Phys 29, 1847-1858 (2002).

${ }^{20}$ J. M. Kapatoes, G. H. Olivera, J. P. Balog, H. Keller, P. J. Reckwerdt, and T. R. Mackie, "On the accuracy and effectiveness of dose reconstruction for tomotherapy," Phys Med Biol 46, 943-966 (2001).

${ }^{21} Y$. De Deene, "Gel dosimetry for the dose verification of intensity modulated radiotherapy treatments," Z. Med. Phys. 12, 77-88 (2002).

${ }^{22}$ R. C. Tailor, V. M. Tello, C. B. Schroy, M. Vossler, and W. F. Hanson, "A generic off-axis energy correction for linac photon beam dosimetry," Med. Phys. 25, 662-667 (1998).

${ }^{23} \mathrm{M}$. Fippel, "Fast Monte Carlo dose calculation for photon beams based on the VMC electron algorithm," Med. Phys. 26, 1466-1475 (1999).

${ }^{24}$ M. K. Fix, H. Keller, P. Ruegsegger, and E. J. Born, "Simple beam models for Monte Carlo photon beam dose calculations in radiotherapy," Med. Phys. 27, 2739-2747 (2000).

${ }^{25}$ M. K. Fix, P. J. Keall, K. Dawson, and J. V. Siebers, "Monte Carlo source model for photon beam radiotherapy: photon source characteristics," Med. Phys. 31, 3106-3121 (2004).

${ }^{26}$ M. Fippel, F. Haryanto, O. Dohm, F. Nusslin, and S. Kriesen, "A virtual photon energy fluence model for Monte Carlo dose calculation," Med. Phys. 30, 301-311 (2003).

${ }^{27} \mathrm{I}$. Kawrakow, M. Fippel, and K. Friedrich, "3D electron dose calculation using a Voxel based Monte Carlo algorithm (VMC)," Med. Phys. 23, 445-457 (1996).

${ }^{28}$ I. Kawrakow, and M. Fippel, "Investigation of variance reduction techniques for Monte Carlo photon dose calculation using XVMC," Phys. Med. Biol. 45, 2163-2183 (2000).

${ }^{29}$ D. A. Low, and J. F. Dempsey, "Evaluation of the gamma dose distribution comparison method," Med. Phys. 30, 2455-2464 (2003).

${ }^{30}$ D. A. Low, W. B. Harms, S. Mutic, and J. A. Purdy, "A technique for the quantitative evaluation of dose distributions," Med. Phys. 25, 656-661 (1998). 


\section{CHAPTER}

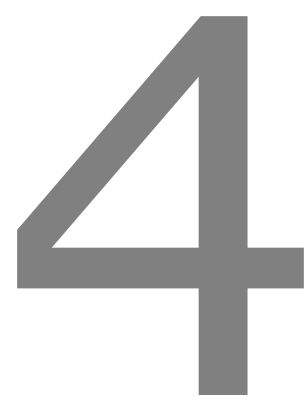

Treatment verification in the presence of inhomogeneities using EPID-based three-dimensional dose reconstruction

Wouter van Elmpt, Sebastiaan Nijsten, André Dekker, Ben Mijnheer and Philippe Lambin

Published in: Medical Physics 34(7):2816-2826, 2007. 


\section{ABSTRACT}

Treatment verification is a prerequisite for the verification of complex treatments, checking both the treatment planning process and the actual beam delivery. Pretreatment verification can detect errors introduced by the treatment planning system (TPS) or differences between planned and delivered dose distributions. In a previous paper we described the reconstruction of three-dimensional (3D) dose distributions in homogeneous phantoms using an in-house developed model based on the beams delivered by the linear accelerator measured with an amorphous silicon electronic portal imaging device (EPID), and a dose calculation engine using the Monte Carlo code $X V M C$. The aim of the present study is to extend the method to situations in which tissue inhomogeneities are present and to make a comparison with the dose distributions calculated by the TPS.

Dose distributions in inhomogeneous phantoms, calculated using the Fast-Fourier Transform Convolution (FFTC) and Multi-Grid Superposition (MGS) algorithms present in the TPS, were verified using the EPID-based dose reconstruction method and compared to film and ionization chamber measurements. Differences between dose distributions were evaluated using the $\gamma$-evaluation method $(3 \% / 3 \mathrm{~mm})$ and expressed as a mean $\gamma$ and the percentage of points with $\gamma>1\left(P_{\gamma>1}\right)$.

For rectangular inhomogeneous phantoms containing a low-density region, the differences between film and reconstructed dose distributions were smaller than $3 \%$. In low-density regions there was an overestimation of the planned dose using the FFTC and MGS algorithms of the TPS up to $20 \%$ and $8 \%$, respectively, for a $10 \mathrm{MV}$ photon beam and a $3 \times 3 \mathrm{~cm}^{2}$ field. For lower energies and larger fields $\left(6 \mathrm{MV}, 5 \times 5 \mathrm{~cm}^{2}\right)$ these differences reduced to $6 \%$ and $3 \%$, respectively. Dose reconstruction performed in an anthropomorphic thoracic phantom for a 3D conformal and an IMRT plan, showed good agreement between film data and reconstructed dose values $\left(P_{\gamma>1}<6 \%\right)$. The algorithms of the TPS underestimated the dose in the low-dose regions outside the treatment field, due to an implementation error of the jaws and MLC of the linac in the TPS. The FFTC algorithm of the TPS showed differences up to $6 \%$ or $6 \mathrm{~mm}$ at the interface between lung and breast. Two IMRT head and neck plans, reconstructed in a commercial phantom having a bone-equivalent insert and an air cavity, showed good agreement between film measurement, reconstructed and planned dose distributions using the FFTC and MGS algorithm, except in the bone-equivalent regions where both TPS algorithms underestimated the dose with $4 \%$. Absolute dose verification was performed at the isocenter where both planned and reconstructed dose were within $2 \%$ of the measured dose. Reproducibility for the EPID measurements was assessed and found to be of negligible influence on the reconstructed dose distribution.

Our 3D dose verification approach is based on the actual dose measured with an EPID in combination with a Monte Carlo dose engine, and therefore independent of a TPS. Because dose values are reconstructed in 3D, isodose surfaces and dose-volume histograms can be used to detect dose differences in target volume and normal tissues. Using our method, the combined planning and treatment delivery process is verified offering an easy to use tool for the verification of complex treatments. 


\section{INTRODUCTION}

Intensity-modulated radiotherapy (IMRT) demands a high accuracy of both the planned dose calculation procedure and of the actual beam delivery by the linear accelerator. Adequate quality control of the dose distribution is necessary for accurate treatments. Most institutions have developed their own strategies for performing IMRT verification. In general, patient-specific quality assurance of (intensitymodulated) radiotherapy can roughly be divided into two different approaches: beamby-beam or total plan verification.

Beam-by-beam verification can be performed in various ways, for instance by using film measurements inside a phantom ${ }^{1-4}$ or using dedicated measurement devices like energy fluence detectors that can be placed on the treatment couch or are attached to the linear accelerator head. Another possibility is to use the electronic portal imaging device (EPID) that is attached permanently to the gantry. ${ }^{5-13}$ The advantage of beam-by-beam verification is that errors or differences are directly related to a particular beam (segment) and the source of the error is easy to detect, for instance a wrong leaf position, deleted or wrong number of monitor units of a segment or deletion of a whole beam. The disadvantage is that these types of errors are not easily related to the total plan, or to the dose in the target volume or in organs at risk.

Total plan verification is an alternative for the beam-by-beam verification procedure. This is often performed by placing a film inside a, usually homogeneous, phantom with or without an ionization chamber and the total plan is delivered to this phantom. The same beam sequence as applied for a specific patient is transferred to this phantom and the dose distribution inside the phantom is calculated using the clinically applied treatment planning system (TPS). Such a "hybrid" plan is evaluated by comparing the calculated and measured dose distribution inside this phantom. The advantage of this procedure is that the effect of possible differences in the individual beams are expressed in terms of the total dose distribution, because differences may add up or cancel out in the total dose distribution. Total plan verification can be performed using a single or a set of multiple films placed in a phantom, using specially developed gels ${ }^{14}$, or by using three-dimensional (3D) dose reconstruction algorithms that relate measured portal images to the dose delivered inside the phantom or patient. $^{15-20}$ The problem with total plan verification using a phantom is that if the treatment plan is transferred to this phantom, all anatomical details of the individual patient are lost. If differences are visible in the dose distribution measured or reconstructed in the phantom situation, it is not always clear how these discrepancies will affect the dose distribution inside the patient, and how these differences can be quantified in terms of dose in the target volume or in organs at risk.

The aim of our work was to develop a procedure that is able to use the actual measured energy fluence of beam segments delivered by the linac, in combination with an accurate dose calculation algorithm, to reconstruct the three-dimensional dose distribution in the patient based on the delivered beams. The energy fluence that is exiting the linac is measured using an EPID and the dose calculation is performed with a dose engine based on Monte Carlo computation, which yields in principle the most accurate dose calculation in inhomogeneous regions. The approach described in this 


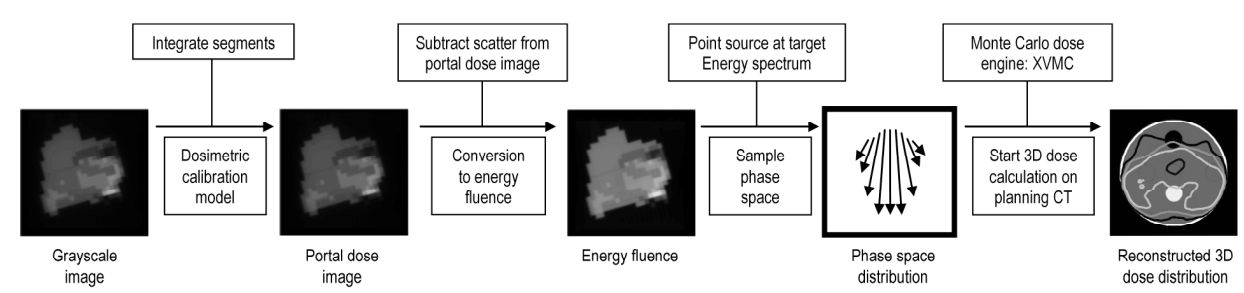

Figure 4.1: Schematic overview of the 3D dose reconstruction model.

work is an extension of a previous developed 3D dose reconstruction method. ${ }^{17}$ The aim of this study is to calculate the dose using a set of planning CT scans, including the full patient anatomy and the presence of tissue inhomogeneities. Various inhomogeneous phantoms are used to verify our model both with conventional and IMRT fields. The dose reconstruction model is compared to film measurements and to dose distributions calculated by the treatment planning system using two different dose calculation algorithms.

\section{MATERIALS AND METHODS}

\section{D dose reconstruction model}

Our 3D dose reconstruction model is based on measured portal images and a dose calculation engine which functions independently of the clinically applied treatment planning system. The procedure has been described in detail in an earlier paper ${ }^{17}$, and will only be addressed briefly.

Treatment fields are measured with an EPID prior to treatment without a phantom or patient in the beam using the same delivery sequence, i.e. applying the same number of monitor units and gantry angles as used for the actual patient treatment. For every beam or gantry angle, the image information of the various segments of a beam is integrated and the measured greyscale images are converted into portal dose images. $^{21,22}$ From these portal dose images the energy fluence is extracted using a deconvolution with a dose-deposition kernel. A phase space is sampled from the energy fluence, assuming that photons exit a point source located at the position of the target. The final step is a dose calculation based on the Monte Carlo code $\mathrm{XVMC}^{23}$ that can be performed using a phantom or the planning CT scan of a patient. A schematic overview of the dose reconstruction process is shown in Figure 4.1.

This dose reconstruction method was validated in a previous study using homogeneous phantoms for both conformal and intensity-modulated fields and showed excellent agreement with measurements. ${ }^{17}$

\section{Linear accelerator and electronic portal imaging device}

Fields are delivered using a Siemens Oncor linear accelerator (Siemens Medical Solutions, Concord, CA, USA) equipped with 6 and $10 \mathrm{MV}$ photons. Two types of EPIDs are used, both amorphous silicon type detectors, only differing in the amount of pixels; 
one detector has $512 \times 512$ pixels (Siemens OptiVue 500) while the other detector has $1024 \times 1024$ pixels (Siemens OptiVue 1000). On top of the sensitive layer of the EPID a $3 \mathrm{~mm}$ copper slab is mounted to remove low energy photons. ${ }^{24,25}$ Images are captured using the standard software of the linear accelerator (Siemens Coherence Workstation). The images of the OptiVue 1000 panel are resampled to a resolution of $512 \times 512$ pixels. The measured portal images are then converted into portal dose images using in-house developed software. ${ }^{22}$ The portal dose images are corrected for ghosting ${ }^{24,25}$, beam profile and the difference in dose in the EPID compared to the water phantom. The EPID accuracy for measuring dose distributions for open and wedged fields, as well as for step-and-shoot IMRT fields, is generally better than 2\% (1 standard deviation (SD)) compared to water phantom measurements. The reproducibility of the EPID acquisition was assessed by measuring a $10 \times 10 \mathrm{~cm}^{2}$ test field each day at the start-up of the linac over a period of 6 months and was better than $0.5 \%$ ( 1 SD) for both 6 and $10 \mathrm{MV}$ photons. ${ }^{22}$

\section{Treatment planning, dose calculation and dose reconstruction}

Dose calculations are performed with our treatment planning system (XiO 4.2.2, Computerized Medical Systems Inc., St. Louis, MO, USA), using both the Fast-Fourier Transform Convolution (FFTC) and the Multi-Grid Superposition (MGS) algorithm. ${ }^{26,27}$ The 3D dose distribution is calculated using the planning CT scan (CT Open, Siemens Medical Solutions, Erlangen, Germany). For both the planning procedure and the dose reconstruction model the same Hounsfield unit to electron density calibration is used. Dose calculations are performed with a grid size of $2 \mathrm{~mm}$ in each direction.

The dose reconstruction procedure uses a grid of $3 \mathrm{~mm}$ to reduce the calculation time, except for simple simulations where a grid size of $2 \mathrm{~mm}$ is used. Dose distributions measured by film or calculated with the dose reconstruction method are interpolated to the grid size of the TPS calculated dose distribution.

The number of sampled photons in the phase-space for the 3D reconstruction procedure was about $2 \times 10^{8}$ photons resulting in a statistical error generally below $2 \%$ per beam. The average time for reconstructing a typical 5-beam plan with a statistical error smaller than $1 \%$ is 5 hours on a dual $1.8 \mathrm{GHz}$ Xeon processor. To compare the reconstructed dose to the planned dose calculated by the treatment planning system and the measured dose with film, the dose calculated by the Monte Carlo code is reported as dose to water. ${ }^{28,29}$

\section{Measurement equipment and dose comparison}

Film measurements are performed using EDR2 films (Eastman Kodak Company, Rochester, NY, USA), processed using a Kodak X-Omat 1000 film processor and digitized using a VXR-16 scanner (Vidar Systems Corporation, Herndon, VA, USA). Calibration films are obtained with the film positioned at the isocenter using $5 \mathrm{~cm}$ of polystyrene build-up for a range of 10 to 250 monitor units (MUs). Absolute dose verification measurements are performed using a calibrated Farmer-type ionization chamber (NE 2505/3, NE Technology Ltd, Reading, UK) read out by a UNIDOS E electrometer (PTW, Freiburg, Germany). 
Dose comparisons are performed using the gamma-evaluation ${ }^{30}$ using a dose criterion of $3 \%$ of the maximum dose and a distance-to-agreement criterion of $3 \mathrm{~mm}$. Statistical parameters such as the mean $\gamma$-value $\left(\gamma_{\text {mean }}\right)$ and the percentage of $\gamma$-values larger than unity $\left(P_{\gamma>1}\right)$ are derived within the $5 \%, 50 \%$ and $80 \%$ of the global maximum of the reconstructed dose distribution. Stock et al. ${ }^{31}$ suggested as criteria for acceptance $\gamma_{\text {mean }}<0.5$ and $P_{\gamma>1}<5 \%$, while rejection was proposed for $\gamma_{\text {mean }}>0.6$ and $P_{\gamma>1}>$ $10 \%$. Intermediate values should result in investigation of the cause of the deviations by looking at, for instance, the size and location of the regions of disagreement and other properties of the dose distribution such as dose profiles and dose difference maps. These criteria are used for the interpretation of IMRT plans delivered to a phantom, and will be used here to evaluate the calculated gamma distributions.

\section{Phantom study}

Three types of phantoms are used in this study and will now be described in more detail.

\section{Lung phantom}

The lung phantom used in this study is made out of slabs of polystyrene $\left(1.03 \mathrm{~g} / \mathrm{cm}^{3}\right)$ and cork $\left(0.25 \mathrm{~g} / \mathrm{cm}^{3}\right)$ with dimensions of $29 \mathrm{~cm} \times 29 \mathrm{~cm}$ and various thicknesses. A slab of cork of $5 \mathrm{~cm}$ is embedded between two layers of polystyrene. The upper and lower polystyrene layer have thicknesses of $7.5 \mathrm{~cm}$ and $12.5 \mathrm{~cm}$, respectively. The source-to-surface distance is $90.0 \mathrm{~cm}$. A film is placed inside this phantom parallel to the central beam axis, and the phantom is pushed tightly together to reduce possible air gaps between the phantom and the film. Measurements are normalized at a point inside the polystyrene region located on the central beam axis at $5 \mathrm{~cm}$ depth. Square field sizes with widths of 3 and $5 \mathrm{~cm}$ were measured for both 6 and $10 \mathrm{MV}$ photon beams using a dose of about $200 \mathrm{cGy}$ delivered at the depth of dose maximum in the phantom. The phantom geometry with density information of the various high- and low-density regions was simulated in the planning system on a contour basis. For this phantom, dose reconstruction based on the measured portal images was performed using the same grid as the planned dose grid, i.e. $2 \times 2 \times 2 \mathrm{~mm}^{3}$.

\section{Thorax phantom}

An anthropomorphic phantom of the thorax region made of polystyrene and cork is used for the evaluation of a clinically used 3D conformal breast treatment plan, consisting of two almost opposing $6 \mathrm{MV}$ photon beams, using a CT scan of this phantom. Field sizes were $9 \times 18 \mathrm{~cm}^{2}$ and $9 \times 17.6 \mathrm{~cm}^{2}$. Inside the phantom 4 film measurements are performed in transversal slices located at $-4.6 \mathrm{~cm},-1.6 \mathrm{~cm}$ cranially and $+1.4 \mathrm{~cm}$ and $+3.5 \mathrm{~cm}$ caudally from the isocenter plane.

Additionally, an IMRT plan for a mediastinal tumor was created on the thorax phantom. The treatment plan consisted of 6 beams with gantry angles 230, 265, 309, 0,51 and 102 degrees (IEC convention) with field sizes modulated within a $10 \times 12 \mathrm{~cm}^{2}$ 
area. Four films were positioned at $-3.6 \mathrm{~cm},-1.2 \mathrm{~cm},+0.6 \mathrm{~cm}$ and $+2.7 \mathrm{~cm}$ from the isocenter plane.

\section{Head and neck phantom}

The head and neck phantom used for the verification of IMRT plans is a commercial phantom (CIRS, Norfolk, VA, USA) in which plugs of various densities can be inserted. The cylindrical phantom is made of water-equivalent material with a $16 \mathrm{~cm}$ diameter and a $30 \mathrm{~cm}$ length. For this study, one insert is filled with bone-equivalent material with a density of $1.60 \mathrm{~g} / \mathrm{cm}^{3}$ and an electron density that is 1.506 times larger relative to that of water. Another insert is left empty simulating an air cavity. A CT scan of this phantom is made and two clinically used IMRT plans of different patients are transferred to this phantom, referred to as plan 1 and plan 2. The treatment technique for both patients consists of 7 beams with gantry angles 206, 258, 309, 0, 51, 102 and 154 degrees (IEC convention). The intensity modulation was optimized within a field size of $20 \times 20 \mathrm{~cm}^{2}$. Two film measurements are made at two transversal positions inside the phantom located at $-1.6 \mathrm{~cm}$ and $+1.6 \mathrm{~cm}$ from the isocenter plane. In addition, an ionization chamber measurement in the center of the phantom is performed for both plans to verify the absolute dose. To verify the reproducibility of the film measurements and the EPID based 3D dose reconstruction, the 2 film measurements and the portal images were acquired again two months later for treatment plan 1 following the same procedure.

\section{RESULTS}

\section{Lung phantom}

Differences between the planned, measured and reconstructed dose distributions have been determined by comparing percentage depth dose curves. In Figure 4.2 percentage depth dose curves (PDDs) are shown for the two algorithms of the TPS, the PDDs extracted from the 3D dose reconstruction and the PDDs resulting from the film measurement for a $3 \times 3 \mathrm{~cm}^{2}$ and $5 \times 5 \mathrm{~cm}^{2}$ field using 6 and $10 \mathrm{MV}$ photon beams. The planned and reconstructed measurements showed good agreement with the measured dose distributions in the high-density regions (polystyrene) for both field sizes and photon beam energies. In the low-density regions (cork) there are differences between planned and measured depth dose curves. The FFTC algorithm overestimates the dose in the low-density regions up to $20 \%$ (local difference) at the distal end of the low-density region for the high photon beam energies and smallest field size, i.e. 10 $M V$ and $3 \times 3 \mathrm{~cm}^{2}$. The MGS algorithm has, for the same setup, the largest local difference of $9 \%$ at the proximal side of the low-density region. For the lower photon beam energy and larger field size, i.e. $6 \mathrm{MV}$ and $5 \times 5 \mathrm{~cm}^{2}$, the maximum difference between planned and measured dose values is below $6 \%$ and $3 \%$, for the FFTC and MGS algorithm, respectively. The reconstructed dose value is within $3 \%$ with respect to the film measurement for all field sizes and photon energies. 

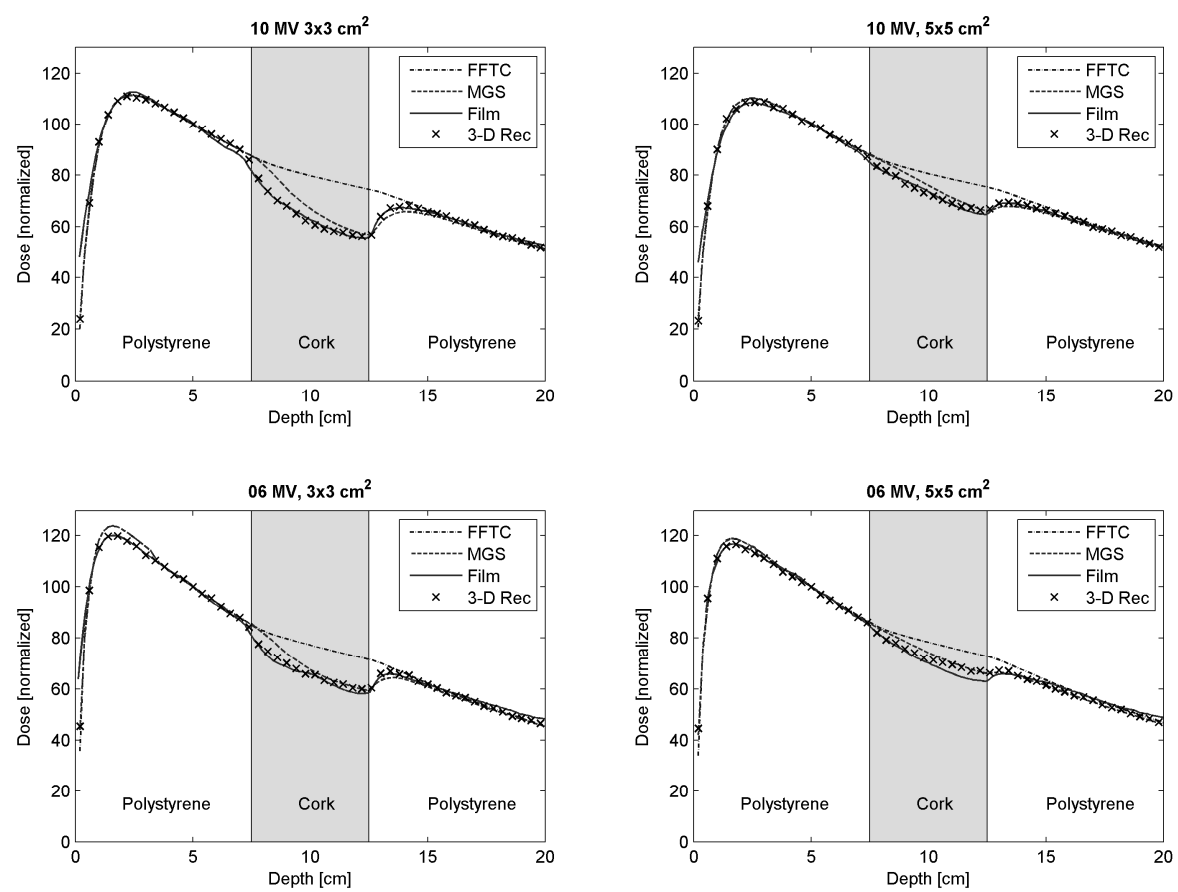

Figure 4.2: Depth dose curves, normalized at $5 \mathrm{~cm}$ depth, of the reconstructed (3-D Rec) and the planned dose distribution using the two algorithms of the planning system (FFTC and MGS) compared to the measured dose distribution using film. Depth dose curves are shown for 6 and $10 \mathrm{MV}$ photon beams and two field sizes, i.e. $3 \times 3 \mathrm{~cm}^{2}$ and $5 \times 5 \mathrm{~cm}^{2}$.

\section{Thorax phantom}

Inside the thorax phantom four films were placed and differences with the TPS calculation and 3D dose reconstruction method were calculated using the gamma-evaluation method. In Figure 4.3, an example of the dose distribution of the 3D conformal breast plan is shown at the position $3.5 \mathrm{~cm}$ caudally from the isocenter. Figure 4.3 shows the calculated, reconstructed and measured dose distribution on top of the corresponding CT slice. The gamma evaluation is used to quantify differences between the various dose distributions. The results of this gamma evaluation averaged over the $4 \mathrm{film}$ measurements performed at different positions are shown in Table 4.1. Statistical analysis for these four films has been performed for different dose regions: the total field within the $5 \%$ and for intermediate and high dose regions within the $50 \%$ and $80 \%$ of the maximum dose in the slice, respectively. Differences are expressed as the percentage of gamma values larger than unity and the mean gamma value. Differences in the low-dose regions are observed for both the FFTC and the MGS algorithm of the TPS and on average there is an underestimation of the dose calculated by the planning system. The local underestimation of the TPS calculated dose is a factor 3 compared to the film dose. Also a disagreement between the dose calculated with the FFTC algo- 


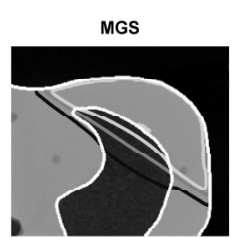

MGS vs. Film

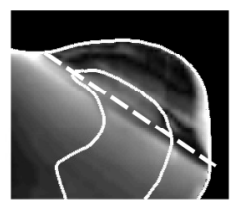

MGS vs. Film

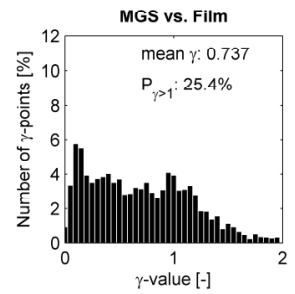

FFTC

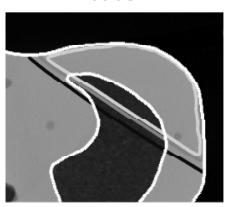

FFTC vs. Film

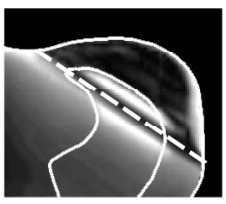

FFTC vs. Film

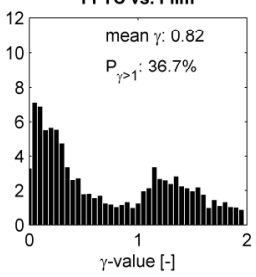

3-D Reconstruction

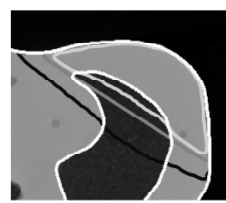

3-D Rec vs. Film
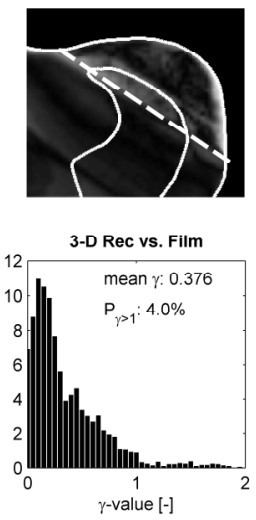
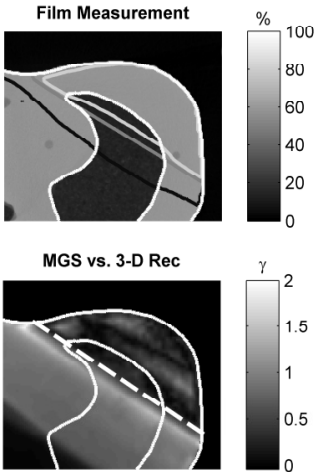

MGS vs. 3-D Rec

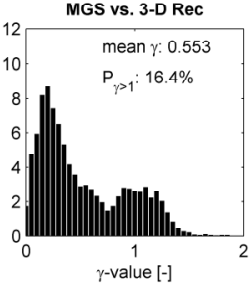

Figure 4.3: Calculated, reconstructed and measured dose distributions and gamma evaluations for a film plane inside the thorax phantom for the 3D conformal plan. The figure represents data in a plane $3.5 \mathrm{~cm}$ caudally from the isocenter plane. Isodose lines are plotted in the upper row for $5 \%, 50 \%$ and $80 \%$ of the maximum dose. For the gamma evaluation a dose criterion of $3 \%$ of the maximum dose and a distance criterion of $3 \mathrm{~mm}$ is used. The dashed white line indicates the $50 \%$ isodose line.

rithm and the film measurement is observed in the lung tissue. Two large regions are visible where the gamma evaluation shows values larger than 2 indicating a dose difference larger than $6 \%$ or a distance-to-agreement larger than $6 \mathrm{~mm}$. These two regions are inside the lung and are separated approximately by the $50 \%$ isodose line.

The results of the gamma evaluation for the IMRT plan delivered to the thorax phantom are averaged over the 4 films at different positions and shown in Table 4.1. An example of the dose distributions for the slice at position $-3.6 \mathrm{~cm}$ is shown in Figure 4.4. This slice is located near the edge of the treatment field, thus the lack of penumbra broadening for the FFTC algorithm is clearly visible in the lung tissue by the large area of high gamma values. The MGS, 3D reconstructed and measured dose distribution are in good agreement with each other. For the slice at the position shown in Figure 4.4, the percentage gamma values above unity and mean gamma value within the area encompassed by $5 \%$ of the maximum dose are $5.4 \%\left(\gamma_{\text {mean }}=0.467\right)$ and $2.8 \%$ $\left(\gamma_{\text {mean }}=0.376\right)$, for the MGS algorithm and the 3D reconstruction, respectively, compared to the film measurement. The FFTC dose distribution shows a large deviation $\left(P_{\gamma>1}=24 \%, \gamma_{\text {mean }}=0.734\right)$ compared to the film measurement for this slice. 
Table 4.1: Analysis of gamma-values of the comparison between the various dose calculation methods and the film measurements for the 3D conformal plan and the IMRT plan delivered to the thorax phantom. Results are expressed as the average values with their standard deviation (SD).

\begin{tabular}{|c|c|c|c|c|c|c|c|}
\hline \multirow[t]{2}{*}{ Thorax Phantom } & & \multicolumn{2}{|c|}{$\begin{array}{c}\text { within } 5 \% \\
\text { isodose line of } \\
\text { maximum dose }\end{array}$} & \multicolumn{2}{|c|}{$\begin{array}{c}\text { within } 50 \% \\
\text { isodose line of } \\
\text { maximum dose }\end{array}$} & \multicolumn{2}{|c|}{$\begin{array}{l}\text { within } 80 \% \\
\text { isodose line of } \\
\text { maximum dose }\end{array}$} \\
\hline & & $\begin{array}{c}Y_{\text {mean }}[-] \\
\text { mean } \\
(1 S D)\end{array}$ & $\begin{array}{l}P_{y>1}[\%] \\
\text { mean } \\
(1 S D)\end{array}$ & $\begin{array}{c}Y_{\text {mean }}[-] \\
\text { mean } \\
\text { (1SD) }\end{array}$ & $\begin{array}{l}P_{\gamma>1}[\%] \\
\text { mean } \\
\text { (1SD) }\end{array}$ & $\begin{array}{c}Y_{\text {mean }}[-] \\
\text { mean } \\
(1 S D)\end{array}$ & $\begin{array}{l}P_{y>1}[\%] \\
\text { mean } \\
(1 S D)\end{array}$ \\
\hline \multirow{4}{*}{$\begin{array}{l}\text { 3D Conformal } \\
\text { Plan (breast) } \\
\text { Average of } 4 \text { film } \\
\text { measurements at } \\
\text { different axial } \\
\text { positions }\end{array}$} & $\begin{array}{c}\text { MGS } \\
\text { vs. Film }\end{array}$ & $\begin{array}{c}0.794 \\
(0.124)\end{array}$ & $\begin{array}{l}31.6 \\
(6.4)\end{array}$ & $\begin{array}{c}0.575 \\
(0.136)\end{array}$ & $\begin{array}{l}11.4 \\
(7.6)\end{array}$ & $\begin{array}{c}0.554 \\
(0.182)\end{array}$ & $\begin{array}{l}10.5 \\
(9.3)\end{array}$ \\
\hline & $\begin{array}{c}\text { FFTC } \\
\text { vs. Film }\end{array}$ & $\begin{array}{c}0.898 \\
(0.162)\end{array}$ & $\begin{array}{l}34.3 \\
(5.8)\end{array}$ & $\begin{array}{c}0.629 \\
(0.195)\end{array}$ & $\begin{array}{l}14.4 \\
(9.3)\end{array}$ & $\begin{array}{c}0.602 \\
(0.243)\end{array}$ & $\begin{array}{c}13.2 \\
(11.0)\end{array}$ \\
\hline & $\begin{array}{l}\text { 3-D Rec. } \\
\text { vs. Film }\end{array}$ & $\begin{array}{c}0.426 \\
(0.089)\end{array}$ & $\begin{array}{l}5.0 \\
(4.2)\end{array}$ & $\begin{array}{c}0.464 \\
(0.082)\end{array}$ & $\begin{array}{l}5.3 \\
\text { (3.9) }\end{array}$ & $\begin{array}{c}0.441 \\
(0.098)\end{array}$ & $\begin{array}{c}4.9 \\
(5.0)\end{array}$ \\
\hline & $\begin{array}{l}\text { 3-D Rec. } \\
\text { vs. MGS }\end{array}$ & $\begin{array}{c}0.559 \\
(0.027)\end{array}$ & $\begin{array}{l}19.1 \\
(1.8)\end{array}$ & $\begin{array}{c}0.304 \\
(0.047)\end{array}$ & $\begin{array}{c}0.3 \\
(0.5)\end{array}$ & $\begin{array}{c}0.303 \\
(0.048)\end{array}$ & $\begin{array}{c}0.3 \\
(0.7)\end{array}$ \\
\hline \multirow{4}{*}{$\begin{array}{c}\text { IMRT Plan } \\
\text { (mediastinum) } \\
\text { Average of } 4 \text { film } \\
\text { measurements at } \\
\text { different axial } \\
\text { positions }\end{array}$} & $\begin{array}{l}\text { MGS } \\
\text { vs. Film }\end{array}$ & $\begin{array}{c}0.516 \\
(0.042)\end{array}$ & $\begin{array}{l}8.0 \\
(2.1)\end{array}$ & $\begin{array}{c}0.451 \\
(0.066)\end{array}$ & $\begin{array}{c}5.3 \\
(1.5)\end{array}$ & $\begin{array}{c}0.432 \\
(0.014)\end{array}$ & $\begin{array}{c}2.6 \\
(1.2)\end{array}$ \\
\hline & $\begin{array}{c}\text { FFTC } \\
\text { vs. Film }\end{array}$ & $\begin{array}{c}0.634 \\
(0.075)\end{array}$ & $\begin{array}{l}16.5 \\
(5.4)\end{array}$ & $\begin{array}{c}0.809 \\
(0.135)\end{array}$ & $\begin{array}{l}28.8 \\
(9.6)\end{array}$ & $\begin{array}{c}1.008 \\
(0.044)\end{array}$ & $\begin{array}{l}42.8 \\
(2.8)\end{array}$ \\
\hline & $\begin{array}{l}\text { 3-D Rec. } \\
\text { vs. Film }\end{array}$ & $\begin{array}{c}0.426 \\
(0.068)\end{array}$ & $\begin{array}{c}6.3 \\
(3.3)\end{array}$ & $\begin{array}{c}0.434 \\
(0.051)\end{array}$ & $\begin{array}{c}4.0 \\
(1.8)\end{array}$ & $\begin{array}{c}0.377 \\
(0.032)\end{array}$ & $\begin{array}{c}1.6 \\
(1.1)\end{array}$ \\
\hline & $\begin{array}{l}\text { 3-D Rec. } \\
\text { vs. MGS }\end{array}$ & $\begin{array}{c}0.372 \\
(0.049)\end{array}$ & $\begin{array}{c}1.2 \\
(1.3)\end{array}$ & $\begin{array}{c}0.356 \\
(0.043)\end{array}$ & $\begin{array}{c}1.5 \\
(1.5)\end{array}$ & $\begin{array}{c}0.324 \\
(0.040)\end{array}$ & $\begin{array}{c}0.3 \\
(0.6)\end{array}$ \\
\hline
\end{tabular}

Acceptance criteria according to Stock et al. ${ }^{31}$ Bold numbers indicate non-acceptable values: $V_{\text {mean }}>0.6$ or $P_{p>1}>10 \%$. Italic numbers fall in the intermediate range, i.e. acceptable if other verification tools indicate no large errors: $0.5>\gamma_{\text {mean }}>0.6$ or $5 \%>P_{\nu>1}>10 \%$.

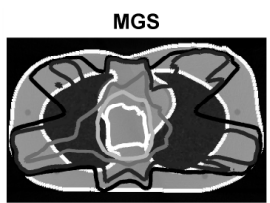

MGS vs. Film

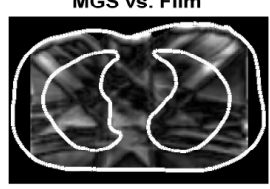

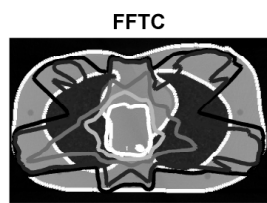

FFTC vs. Film

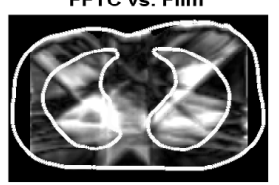

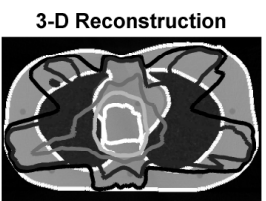

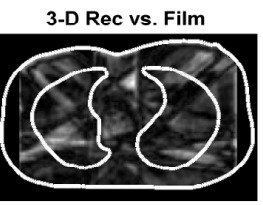

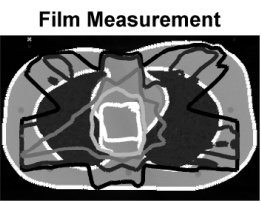
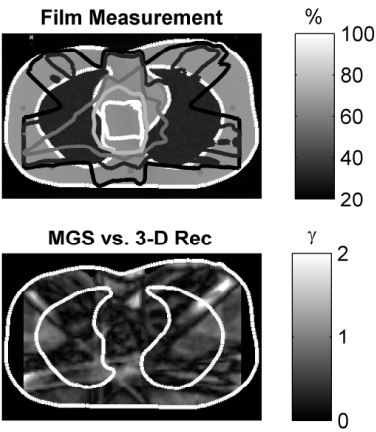

Figure 4.4: Results of the IMRT plan delivered to the thorax phantom. Isodose lines for $20 \%, 40 \%$, $60 \%, 70 \%$ and $90 \%$ of the maximum dose are shown. The bottom row shows the gamma evaluation indicating that the film size does not cover the total phantom and the edges are not taken into account during the gamma evaluation. 


\section{Head and neck phantom}

The measured energy fluence distributions for plan 1 are shown in Figure 4.5. For this plan, the measured dose with the ionization chamber at the isocenter for a single fraction was $2.13 \mathrm{~Gy}$ compared to $2.11 \mathrm{~Gy}$ for the dose reconstruction and $2.18 \mathrm{~Gy}$ for both the FFTC and the MGS algorithm of the TPS. For plan 2, the absolute dose values were 1.82, 1.81 and 1.84 Gy for the TPS algorithms, the dose reconstruction and the ionization chamber measurement, respectively. All dose calculations and dose reconstructions are within $3 \%$ of the measured dose values.

The calculated, reconstructed and measured dose distributions for plan 1 are shown in Figure 4.6 in which isodose lines are plotted for $50 \%, 60 \%, 70 \%$ and $80 \%$ of the maximum dose delivered. The air cavity is excluded from the gamma analysis due to the fact that the presence of the film disturbs the dose distribution in the air cavity. Statistical data of the gamma comparison averaged for the 4 films of plan 1 and the results for the 2 films of plan 2 are shown in Table 4.2. Mean gamma values and the percentage of gamma values larger than unity are shown.

For plan 1, good agreement with the 4 film measurements is observed, i.e. $P_{\gamma>1}$ smaller than $2 \%$ and $\gamma_{\text {mean }}<0.4$ for both TPS dose calculations and the 3D dose reconstruction. However, a large region of disagreement for the algorithms of the TPS is visible around the bone insert. A systematic underestimation of the dose of $4 \%$ occurs in this region, resulting in a $\gamma_{\text {mean }}$ of 0.96 and 1.01 for the MGS and the FFTC algorithm, respectively. The 3D reconstructed dose based on Monte Carlo shows a smaller difference in this region $\left(\gamma_{\text {mean }}=0.46\right)$ compared to the film measurement.

For plan 2 the differences between the 2 film measurements and the dose reconstruction or dose calculation are somewhat larger but generally $\mathrm{P}_{\gamma>1}$ is around $5 \%$ with $\gamma_{\text {mean }}$ lower than 0.5 . This plan shows more modulation in the delivered dose distribution than plan 1 which possibly explains the larger differences in gamma statistics compared to the more homogeneous dose distribution of plan 1.

The reproducibility of the film measurements was evaluated by repeating the entire film measurement procedure for plan 1 approximately 2 months later. No significant differences were observed either in the delivery by the linear accelerator or in the measurement and processing results of the 2 films. The individual film results in Table 4.2 for plan 1 are therefore combined and the average of the 4 films is shown.

The reproducibility of the 3D reconstruction based on the EPID measurements was assessed by repeating the acquisition of the portal images and the reconstruction of the 3D dose distribution. A gamma evaluation of the two reconstructed dose distributions in the corresponding slices that encompassed the entire 3D dose distribution was performed at different times showing very good agreement $\left(P_{\gamma>1}=0.8 \%\right)$. Such a comparison includes possible differences in output of the linear accelerator and possible aging of the EPID detector that could result in a difference in response. 


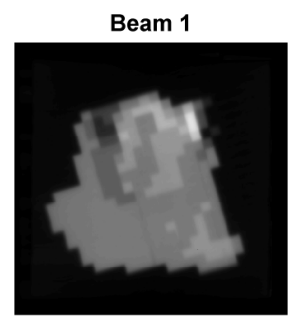

Beam 5

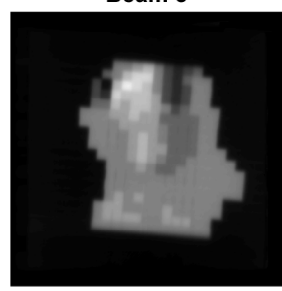

Beam 2

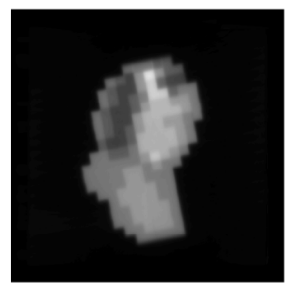

Beam 6

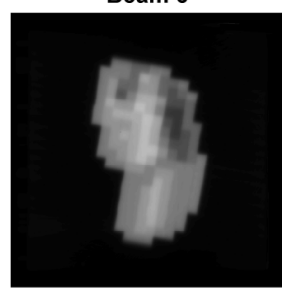

Beam 3

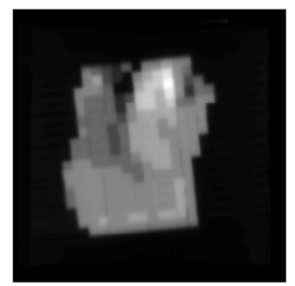

Beam 7

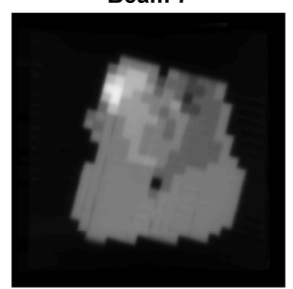

Beam 4

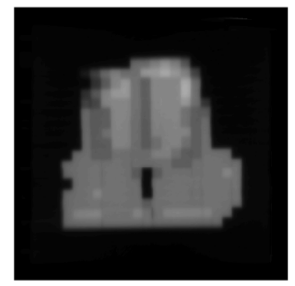

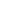

.
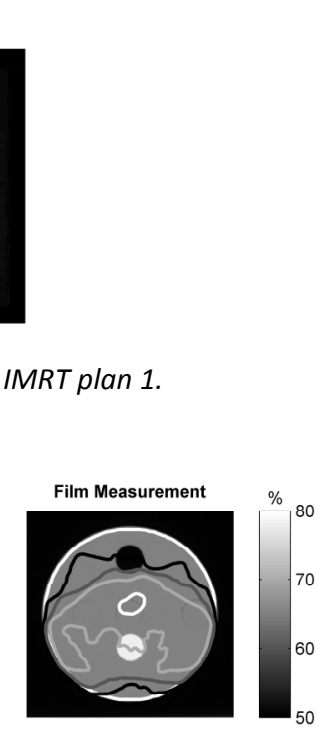

Figure 4.5: Extracted energy fluence distributions of the head and neck IMRT plan 1.
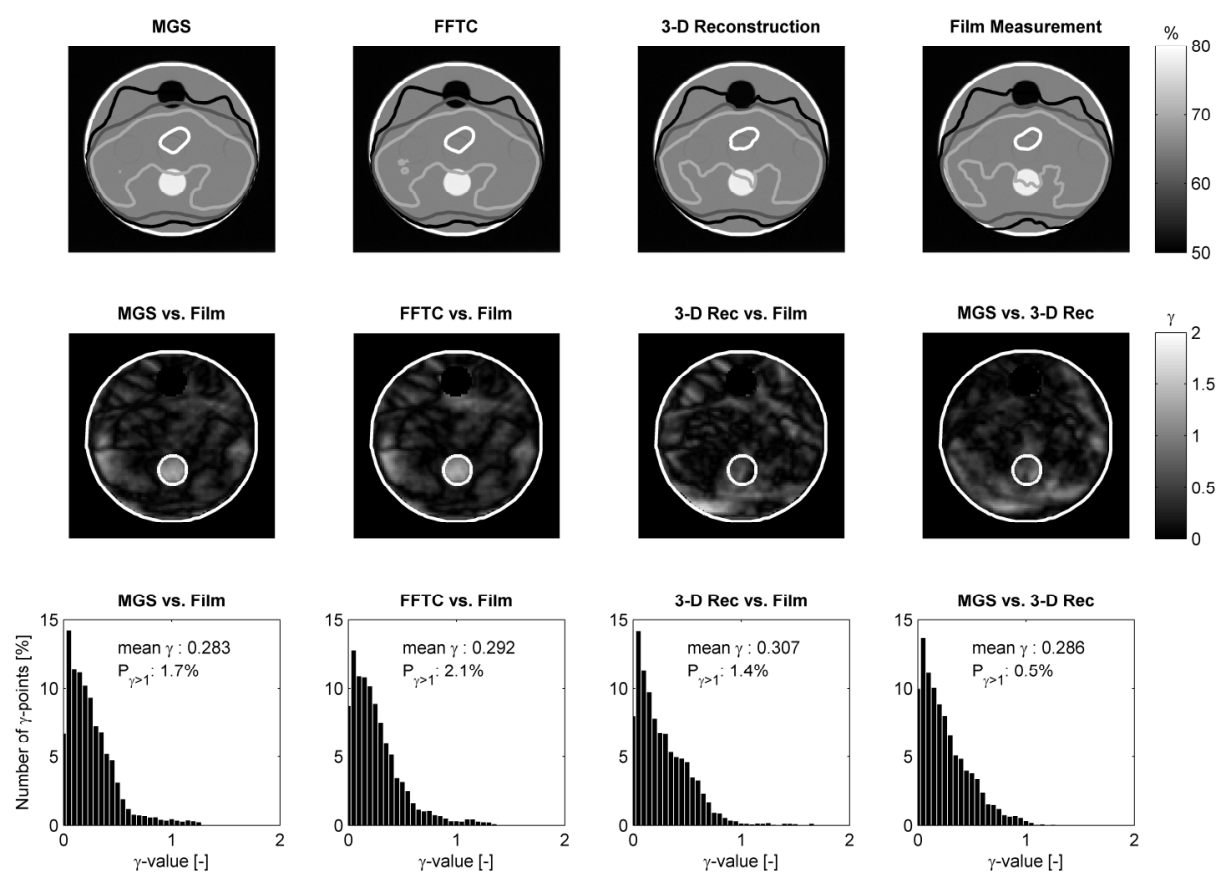

Figure 4.6: Dose distribution, gamma plots and gamma histograms in a plane located at -1.6 $\mathrm{cm}$ from the isocenter, for the head and neck phantom of plan 1. Isodose lines are shown for the $50 \%, 60 \%, 70 \%$ and $80 \%$ of dose maximum. The gamma histograms are calculated over the area within the $5 \%$ isodose line excluding the air cavity, with a $3 \%$ of the maximum dose criterion and a $3 \mathrm{~mm}$ distance-to-agreement criterion. 
Table 4.2: Analysis of gamma-values of the comparison between the various dose calculation methods and the film measurements for both plans delivered to the head and neck phantom. Results are expressed as the average and standard deviation (SD) of the four films of plan 1 , and as the individual values for the two film measurements of plan 2.

\begin{tabular}{|c|c|c|c|c|c|c|c|}
\hline \multirow[t]{2}{*}{$\begin{array}{l}\text { Head and Neck } \\
\text { Phantom }\end{array}$} & & \multicolumn{2}{|c|}{$\begin{array}{c}\text { within } 5 \% \\
\text { isodose line of } \\
\text { maximum dose }\end{array}$} & \multicolumn{2}{|c|}{$\begin{array}{l}\text { within } 50 \% \\
\text { isodose line of } \\
\text { maximum dose }\end{array}$} & \multicolumn{2}{|c|}{$\begin{array}{c}\text { within } \mathbf{8 0 \%} \\
\text { isodose line of } \\
\text { maximum dose }\end{array}$} \\
\hline & & $V_{\text {mean }}[-]$ & $P_{\gamma>1}[\%]$ & $V_{\text {mean }}[-]$ & $P_{Y>1}[\%]$ & $V_{\text {mean }}[-]$ & $P_{\gamma>1}[\%]$ \\
\hline \multirow{6}{*}{$\begin{array}{l}\text { IMRT Plan } 1 \\
\text { Results of } 4 \text { film } \\
\text { measurements: } \\
\text { mean (1SD) }\end{array}$} & $\begin{array}{l}\text { MGS vs. } \\
\text { Film }\end{array}$ & $\begin{array}{c}0.341 \\
(0.078)\end{array}$ & $\begin{array}{c}1.8 \\
(1.1)\end{array}$ & $\begin{array}{c}0.330 \\
(0.064)\end{array}$ & $\begin{array}{c}1.2 \\
(0.9)\end{array}$ & $\begin{array}{c}0.295 \\
(0.082)\end{array}$ & $\begin{array}{c}0.6 \\
(0.9)\end{array}$ \\
\hline & FFTC vs. & 0.324 & 1.8 & 0.339 & 1.6 & 0.301 & 0.8 \\
\hline & Film & $(0.075)$ & (1.1) & (0.059) & (1.1) & $(0.080)$ & $(1.2)$ \\
\hline & 3-D Rec. & 0.299 & 1.5 & 0.299 & 0.9 & 0.242 & 0.3 \\
\hline & vs. Film & $(0.075)$ & (1.3) & (0.055) & (1.1) & (0.091) & $(0.5)$ \\
\hline & $\begin{array}{l}\text { 3-D Rec. } \\
\text { vs. MGS }\end{array}$ & $\begin{array}{c}0.266 \\
(0.016)\end{array}$ & $\begin{array}{c}0.6 \\
(0.4)\end{array}$ & $\begin{array}{c}0.276 \\
(0.018)\end{array}$ & $\begin{array}{c}0.6 \\
(0.4)\end{array}$ & $\begin{array}{c}0.214 \\
(0.035)\end{array}$ & $\begin{array}{c}0.1 \\
(0.2)\end{array}$ \\
\hline \multirow{4}{*}{$\begin{array}{l}\text { IMRT Plan } 2 \\
\text { Results of } 2 \text { film } \\
\text { measurements: } \\
\text { Film } 1 \text { / Film } 2\end{array}$} & $\begin{array}{l}\text { MGS vs. } \\
\text { Film }\end{array}$ & $\begin{array}{c}0.467 / \\
0.363\end{array}$ & $\begin{array}{c}5.7 / \\
1.6\end{array}$ & $\begin{array}{c}0.464 / \\
0.375\end{array}$ & $\begin{array}{c}5.4 / \\
2.7\end{array}$ & $\begin{array}{c}0.525 / \\
0.441\end{array}$ & $\begin{array}{c}5.0 / \\
4.5\end{array}$ \\
\hline & $\begin{array}{l}\text { FFTC vs. } \\
\text { Film }\end{array}$ & $\begin{array}{c}0.478 / \\
0.367\end{array}$ & $\begin{array}{c}6.0 / \\
2.1\end{array}$ & $\begin{array}{c}0.470 / \\
0.397\end{array}$ & $\begin{array}{c}6.0 / \\
3.7\end{array}$ & $\begin{array}{c}0.545 / \\
0.500\end{array}$ & $\begin{array}{c}6.6 / \\
6.6\end{array}$ \\
\hline & $\begin{array}{l}\text { 3-D Rec. } \\
\text { vs. Film }\end{array}$ & $\begin{array}{c}0.395 / \\
0.363\end{array}$ & $\begin{array}{c}4.6 / \\
1.1\end{array}$ & $\begin{array}{c}0.372 / \\
0.372\end{array}$ & $\begin{array}{l}2.8 / \\
1.7\end{array}$ & $\begin{array}{c}0.407 / \\
0.388\end{array}$ & $\begin{array}{c}4.4 / \\
2.5\end{array}$ \\
\hline & $\begin{array}{l}\text { 3-D Rec. } \\
\text { vs. MGS }\end{array}$ & $\begin{array}{c}0.579 / \\
0.243\end{array}$ & $\begin{array}{c}14.0 / \\
0.16\end{array}$ & $\begin{array}{c}0.588 / \\
0.269\end{array}$ & $\begin{array}{c}15.0 / \\
0.1\end{array}$ & $\begin{array}{c}0.576 / \\
0.335\end{array}$ & $\begin{array}{c}11.0 / \\
0.3\end{array}$ \\
\hline
\end{tabular}

\section{DISCUSSION}

\section{Lung phantom}

For the simple inhomogeneous phantom containing a slab of cork the dose distribution calculated with our dose reconstruction method is in good agreement with measurements. The dose calculation algorithms of the treatment planning system calculate the dose accurately inside the water-equivalent density regions but overestimate the dose inside the low-density regions. This is in agreement with what can be expected from algorithms having these simple inhomogeneity corrections, and was, for instance, also shown in similar studies performed by Miften et al. ${ }^{26,27}$, and in AAPM Report $85 .{ }^{32}$

A small difference for the $3 \times 3 \mathrm{~cm}^{2}$ field was visible in the first $3 \mathrm{~cm}$ of the PDD calculated by the treatment planning system for the $6 \mathrm{MV}$ beam and was mainly due to a misfit of the electron contamination in the TPS. The electron contamination was estimated based on the difference between a measured and a TPS calculated PDD. Verification measurements showed that the measured PDD used during commissioning was not correct. A small misalignment of the central-axis and the measurementaxis probably caused this difference. Other field sizes did not show this overestimation in the region around the dose maximum of the PDD. 


\section{Thorax phantom}

The thorax phantom is an anthropomorphic phantom and includes soft tissue and a low-density region simulating the lung. Treatment planning systems generally have difficulties in calculating the dose distributions for such geometries and density variations. Although for simple pencil beam or convolution algorithms the dose in the target volume is usually correct, as discussed for instance in AAPM Report 85, the dose calculated in the lung tissue may not always be correct. ${ }^{32}$

In Figure 4.3, the two major discrepancies are clearly visible between the TPS calculated dose and the reconstructed or measured dose distribution. First, for the FFTC algorithm there are differences close to the penumbra in the lung tissue. The penumbra broadening effect visible in the film measurement is not calculated accurately with the FFTC algorithm while the MGS algorithm does model this effect more accurately. Second, the dose transmitted through the MLC is not correctly predicted. This is due to the fact that the TPS assumes that there is a pair of backup jaws behind the MLC to reduce the transmission through the MLC while for the Siemens linacs this pair of backup jaws is removed and the blocking of the field in the direction of the MLC leaves is performed only by the MLC. A solution to this problem would be a more accurate representation of the treatment head of the Siemens linac in the XiO planning system. In the current version of XiO (4.2.2) there are two collimator jaws that both have the same transmission value and it is not possible to set this transmission value for the collimator jaws independently.

The reconstructed 3D dose distribution for the 3D conformal and the IMRT plans are in good agreement with the measured dose distribution, both inside and outside the high-dose gradient region. The fact that the measured portal dose image is used as an input for the 3D dose reconstruction removes errors such as a wrong implementation of the collimator and MLC transmission values. The measured energy fluence is directly used to calculate the dose in the CT scan.

The result of the comparison shown in Table 4.1 and Table 4.2 between the MGS algorithm of the TPS with the reconstructed dose values is one of the most interesting findings of this study. These values will be used in the future for accepting or rejecting a treatment plan calculation and delivery. The gamma evaluation of these two dose distributions shows smaller differences than the separate comparisons with the film measurement. This may be due to an error in the film measurement which is not present in the comparison between planned and reconstructed dose distribution. As can be seen for example in Figure 4.3, at the left side of the breast there is a small region with high gamma values in the three comparisons with film. The fact that this is not visible in either the reconstructed or the planned dose distribution suggests that this is possibly an inaccuracy in the film measurement. This may be caused by the film having a size which was not adjusted to the phantom dimensions and extended outside the phantom, thus causing some extra scattered or build-up dose in this region, as observed, for instance by Gilles et al. ${ }^{33}$ However, the experiment was designed to avoid the positioning of a film at central axis of the beam, so that the influence of the part of the film that extended outside the phantom was minimized. Another explanation might be that the phantom is made of $1 \mathrm{~cm}$ slices that are not smoothly overlap- 
ping at the border of the slices, so a small difference in thickness can be present at both sides of the film.

\section{Head and neck phantom}

The head and neck phantom can be used for the verification of the IMRT head and neck plans and delivery. The recommended procedure is to place a film and an ionization chamber inside this phantom, checking both a relative $2 \mathrm{D}$ dose profile and the absolute dose delivered to a single point. Our 3D dose reconstruction procedure is able to perform the same verification without the labour intensive work of setting up the phantom, placing the film and ionization chamber, processing and scanning the film. The input for the dose reconstruction is the measured grayscale image of each beam. Our results show that this reconstruction is in good agreement with the dose that would be measured with both film and ionization chamber.

For plan 1, there are differences visible inside the region with the bone-equivalent density between the TPS calculated dose and the measured dose using film. The dose is underestimated in this region, which may be due to the extra amount of scattered photons and electrons inside the bone-equivalent material that are not accurately taken into account by the algorithms of the TPS. These algorithms do not model the effect of different atomic compositions on the dose delivery but only take into account differences in electron density, Monte Carlo calculations do take into account the difference in cross-sections and stopping powers for different atomic compositions. This may be of clinical importance because the organ at risk in this region is the spinal cord and clinical plans can be rejected or accepted based on the dose in this organ. The 3D dose reconstruction does not show this difference and is in better agreement with the film, as can be expected from a Monte Carlo simulation that inherently takes into account the variation in atomic composition of the material and secondary scattered photons and electrons.

\section{General discussion}

The advantage of our verification procedure compared to the TPS planned dose distribution is that the reconstructed dose distribution gives a more accurate representation of what actually will be delivered to the patient. For instance, possible commissioning errors in the TPS modelling of interleaf leakage, rounded leaf ends, wrong collimator jaw transmission or tongue-and-groove parameters, are not present in the $3 \mathrm{D}$ dose reconstruction because an actual measurement of the energy fluence delivered by the linac is used as input for the 3D dose reconstruction. By using a Monte Carlo dose engine for the patient dose calculation, limitations due to the type of dose calculation algorithm such as the use of pencil beams or a simple convolution model are also removed.

There are two items that can be checked with pre-treatment verification. The first is the accurate delivery of the beam sequence as planned by the TPS, such as positioning of the multi-leaf collimator, delivery of the various segments with proper number of monitor units, as well as the correct treatment parameter transfer from TPS to linac. The second item that can be verified is the dose calculation by the TPS. This dose 
calculation is not always accurate for all situations, e.g. due to the presence of air cavities in the head and neck region or low-density lung tissue in the thorax. Also machine-related parameters must be incorporated accurately, such as tongue-andgroove effects of the MLC, interleaf leakage and block transmission.

If there are differences between planned and measured or reconstructed dose distributions, the first thing that has to be found out is the type of error that has occurred. In case of a failure of the treatment device this has to be fixed before the start of treatment of a patient. If the error is due to an erroneous calculation or a wrong implementation of the linac characteristics in the TPS then this should if possible be detected and removed. However, differences due to a rather simple dose calculation algorithm such as FFTC or pencil beam algorithms cannot be taken into account during the planning procedure. The only thing one can do in such a situation is to be aware of the limitations of the software of the TPS and if possible to avoid or minimize situations in which these errors may occur. For prostate planning, simple algorithms can be sufficient but for sites incorporating tissue inhomogeneities like lung or head and neck, a more accurate algorithm might be necessary. One of the benefits of the proposed pre-treatment verification is that it provides an additional check of the dose that will be delivered to the patient before treatment, although at this late stage making a new plan may delay patient treatment which is not desirable but possible, if necessary.

The overall time needed to perform our verification procedure does not differ much from the time required for conventional IMRT QA using film and ionization chamber dosimetry. What does differ is the user-time that is needed for performing the actual measurements. If one assumes that for conventional IMRT verification 30 minutes are needed to position a phantom, set-up the ionization chamber equipment, insert the film and start the beam delivery, and that an additional 90 minutes are required for film processing and analyzing the film measurements, the total procedure lasts about 2 hours. The 3D dose reconstruction method demands only time for delivering the fields and to measure them with the EPID, which takes less than 20 min. After the acquisition of the portal images, the reconstruction can be started automatically, taking typically some 5 hours for a statistical error below $1 \%$, or less than one hour for a statistical accuracy of $2 \%$. The analysis of the various gamma-evaluations can be performed in $10 \mathrm{~min}$. This analysis can be automated even further if decision rules are implemented.

Because the dose distribution is reconstructed in $3 \mathrm{D}$ in the planning $\mathrm{CT}$, various methods for the evaluation of the reconstructed and planned dose distributions can be used, such as dose-volume histograms (DVHs), the dose in the target volume, gamma histograms or gamma images. The evaluation tools like isodose surfaces and DVHs can be used in the same way as applied during the routine treatment planning process. The application of these tools in clinical practice is the subject of further studies.

While careful analysis of gamma images can lead to valuable insights, in clinical practice clear pass or fail criteria are useful. Stock et al. ${ }^{31}$ suggested criteria for the interpretation of gamma values for hybrid IMRT plans (i.e. plans delivered to a phantom). The accepted, intermediate and rejected values for two parameters according to their classification are used in our analysis and shown in Table 4.1 and Table 4.2. The agreement between the reconstructed dose values and film measurements is excellent for the IMRT head-and-neck treatment. For dose reconstruction inside such a phantom 
geometry maybe more stringent criteria than $3 \% / 3 \mathrm{~mm}$ can therefore be applied because possible errors and inaccuracies in the film measurement are removed.

For treatment verification, this 3D dose reconstruction will be a major step forward compared to the hybrid IMRT phantom verification procedures because we now have the ability to estimate the possible sources of uncertainty in terms of patient related characteristics, such as dose in the target volume or dose in normal tissues. Currently, this 3D dose reconstruction procedure is only used in our institution for the evaluation of complex plans, but we are working on the implementation of our 3D dose reconstruction procedure as a quality control procedure for all our IMRT patients.

\section{CONCLUSION}

We developed a three-dimensional dose reconstruction model that is able to accurately calculate the dose distribution in phantoms or patients based on the actual delivered beams. Incorporating a Monte Carlo dose engine assures a highly accurate dose calculation inside the planning $\mathrm{CT}$ also for regions with inhomogeneous tissue densities. The reconstructed dose distribution can be evaluated in the same way as performed during the treatment planning process, while in addition a rapid evaluation of the differences with the planned dose distribution is possible.

\section{REFERENCES}

${ }^{1}$ X.R. Zhu, P.A. Jursinic, D.F. Grimm, F. Lopez, J.J. Rownd, and M.T. Gillin, "Evaluation of Kodak EDR2 film for dose verification of intensity modulated radiation therapy delivered by a static multileaf collimator," Med. Phys. 29, 1687-1692 (2002).

${ }^{2}$ M. Bucciolini, F.B. Buonamici, and M. Casati, "Verification of IMRT fields by film dosimetry," Med. Phys. 31, 161-168 (2004).

${ }^{3}$ G.J. Budgell, B.A. Perrin, J.H.L. Mott, J. Fairfoul, and R.I. Mackay, "Quantitative analysis of patient-specific dosimetric IMRT verification," Phys. Med. Biol. 50, 103-119 (2005).

${ }^{4}$ A.J. Olch, "Dosimetric performance of an enhanced dose range radiographic film for intensitymodulated radiation therapy quality assurance," Med. Phys. 29, 2159-2168 (2002).

${ }^{5}$ S.M.J.J.G. Nijsten, A.W.H. Minken, P. Lambin, and I.A.D. Bruinvis, "Verification of treatment parameter transfer by means of electronic portal dosimetry," Med. Phys. 31, 341-347 (2004).

${ }^{6}$ K.L. Pasma, B.J.M. Heijmen, M. Kroonwijk, and A.G. Visser, "Portal dose image (PDI) prediction for dosimetric treatment verification in radiotherapy. I. An algorithm for open beams," Med. Phys. 25, 830-840 (1998).

${ }^{7}$ K.L. Pasma, M. Kroonwijk, J.C.J. de Boer, A.G. Visser, and B.J.M. Heijmen, "Accurate portal dose measurement with a fluoroscopic electronic portal imaging device (EPID) for open and wedged beams and dynamic multileaf collimation," Phys. Med. Biol. 43, 2047-2060 (1998).

${ }^{8}$ A. Van Esch, T. Depuydt, and D.P. Huyskens, "The use of an aSi-based EPID for routine absolute dosimetric pre-treatment verification of dynamic IMRT fields," Radiother. Oncol. 71, 223234 (2004).

${ }^{9}$ B.M. McCurdy, K. Luchka, and S. Pistorius, "Dosimetric investigation and portal dose image prediction using an amorphous silicon electronic portal imaging device," Med. Phys. 28, 911-924 (2001). 
${ }^{10}$ W.J.C. van Elmpt, S.M.J.J.G. Nijsten, B.J. Mijnheer, and A.W.H. Minken, "Experimental verification of a portal dose prediction model," Med. Phys. 32, 2805-2818 (2005).

${ }^{11}$ R. Boellaard, M. Essers, M. van Herk, and B.J. Mijnheer, "New method to obtain the midplane dose using portal in vivo dosimetry," Int. J. Radiat. Oncol. Biol. Phys. 41, 465-474 (1998).

${ }^{12}$ M. Wendling, R.J.W. Louwe, L.N. McDermott, J.J. Sonke, M. van Herk, and B.J. Mijnheer, "Accurate two-dimensional IMRT verification using a back-projection EPID dosimetry method," Med. Phys. 33, 259-273 (2006).

${ }^{13}$ B. Warkentin, S. Steciw, S. Rathee, and B.G. Fallone, "Dosimetric IMRT verification with a flatpanel EPID," Med. Phys. 30, 3143-3155 (2003).

${ }^{14} Y$. De Deene, "Gel dosimetry for the dose verification of intensity modulated radiotherapy treatments," Z. Med. Phys. 12, 77-88 (2002).

${ }^{15}$ M. Partridge, M. Ebert, and B.M. Hesse, "IMRT verification by three-dimensional dose reconstruction from portal beam measurements," Med. Phys. 29, 1847-1858 (2002).

${ }^{16}$ R.J.W. Louwe, E.M.F. Damen, M. van Herk, A.W.H. Minken, O. Torzsok, and B.J. Mijnheer, "Three-dimensional dose reconstruction of breast cancer treatment using portal imaging," Med. Phys. 30, 2376-2389 (2003).

${ }^{17}$ W.J.C. van Elmpt, S.M.J.J.G. Nijsten, R.F.H. Schiffeleers, A.L.A.J. Dekker, B.J. Mijnheer, P. Lambin, and A.W.H. Minken, "A Monte Carlo based three-dimensional dose reconstruction method derived from portal dose images," Med. Phys. 33, 2426-2434 (2006).

${ }^{18}$ S. Steciw, B. Warkentin, S. Rathee, and B.G. Fallone, "Three-dimensional IMRT verification with a flat-panel EPID," Med. Phys. 32, 600-612 (2005).

${ }^{19}$ W.D. Renner, M. Sarfaraz, M.A. Earl, and C.X. Yu, "A dose delivery verification method for conventional and intensity-modulated radiation therapy using measured field fluence distributions," Med. Phys. 30, 2996-3005 (2003).

${ }^{20}$ J.M. Kapatoes, G.H. Olivera, J.P. Balog, H. Keller, P.J. Reckwerdt, and T.R. Mackie, "On the accuracy and effectiveness of dose reconstruction for tomotherapy," Phys. Med. Biol. 46, 943-966 (2001).

${ }^{21}$ J. Chen, C.F. Chuang, O. Morin, M. Aubin, and J. Pouliot, "Calibration of an amorphous-silicon flat panel portal imager for exit-beam dosimetry," Med. Phys. 33, 584-594 (2006).

${ }^{22}$ S.M.J.J.G. Nijsten, W.J.C. Van Elmpt, M. Jacobs, B.J. Mijnheer, A.L.A.J. Dekker, P. Lambin, and A.W.H. Minken, "A global correction model of a-Si EPIDs used for transit dosimetry," Med. Phys. in press (2007).

${ }^{23} \mathrm{M}$. Fippel, "Fast Monte Carlo dose calculation for photon beams based on the VMC electron algorithm," Med. Phys. 26, 1466-1475 (1999).

${ }^{24}$ L.N. McDermott, S.M.J.J.G. Nijsten, J.J. Sonke, M. Partridge, M. van Herk, and B.J. Mijnheer, "Comparison of ghosting effects for three commercial a-Si EPIDs," Med. Phys. 33, 24482451 (2006).

${ }^{25}$ L.N. McDermott, R.J.W. Louwe, J.J. Sonke, M.B. van Herk, and B.J. Mijnheer, "Dose-response and ghosting effects of an amorphous silicon electronic portal imaging device," Med. Phys. 31, 285-295 (2004).

${ }^{26} \mathrm{M}$. Miften, M. Wiesmeyer, S. Monthofer, and K. Krippner, "Implementation of FFT convolution and multigrid superposition models in the FOCUS RTP system," Phys. Med. Biol. 45, 817833 (2000).

${ }^{27}$ M. Miften, M. Wiesmeyer, A. Kapur, and C.M. Ma, "Comparison of RTP dose distributions in heterogeneous phantoms with the BEAM Monte Carlo simulation system," J. Appl. Clin. Med. Phys. 2, 21-31 (2001).

${ }^{28}$ J.V. Siebers, P.J. Keall, A.E. Nahum, and R. Mohan, "Converting absorbed dose to medium to absorbed dose to water for Monte Carlo based photon beam dose calculations," Phys. Med. Biol. 45, 983-995 (2000). 
${ }^{29}$ N. Dogan, J.V. Siebers, and P.J. Keall, "Clinical comparison of head and neck and prostate IMRT plans using absorbed dose to medium and absorbed dose to water," Phys. Med. Biol. 51, 4967-4980 (2006).

${ }^{30}$ D.A. Low, W.B. Harms, S. Mutic, and J.A. Purdy, "A technique for the quantitative evaluation of dose distributions," Med. Phys. 25, 656-661 (1998).

${ }^{31} \mathrm{M}$. Stock, B. Kroupa, and D. Georg, "Interpretation and evaluation of the gamma index and the gamma index angle for the verification of IMRT hybrid plans," Phys. Med. Biol. 50, 399-411 (2005).

${ }^{32}$ N. Papanikolaou, J. Battista, A. Boyer, C. Kappas, E. Klein, T.R. Mackie, M. Sharpe, and J. Van Dyk, Tissue Inhomogeneity Corrections for Megavoltage Photon Beams. AAPM Report No 85, Task Group No 65 of the Radiation Therapy Committee of the American Association of Physicists in Medicine (Medical Physics Publishing, Madison, WI, 2004).

${ }^{33}$ S. Gillis, C. de Wagter, J. Bohsung, B. Perrin, P. Williams, and B.J. Mijnheer, "An inter-centre quality assurance network for IMRT verification: Results of the ESTRO QUASIMODO project," Radiother. Oncol. 76, 340-353 (2005). 



\section{CHAPTER}

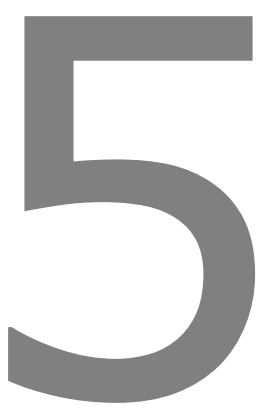

The next step in patient-specific QA: $3 \mathrm{D}$ dose verification of conformal and intensitymodulated RT based on EPID dosimetry and Monte Carlo dose calculations

Wouter van Elmpt, Sebastiaan Nijsten, Ben Mijnheer, André Dekker and Philippe Lambin

Published in: Radiotherapy and Oncology 86(1):86-92, 2008. 
98 Chapter 5

\begin{abstract}
Background and purpose: A method was evaluated to reconstruct the 3D dose distribution in patients using their planning CT scan in combination with a Monte Carlo calculation, and the energy fluence of the actual treatment beams measured pretreatment with an EPID without the patient or a phantom in the beam.

Materials and methods: Nine plans of lung cancer patients treated with a 3D conformal technique, calculated using a simple convolution algorithm (CA), as well as five IMRT treatments of head-and-neck cancer patients, calculated with a more advanced superposition algorithm (SA), were verified. Differences between planned and reconstructed dose distributions were quantified in terms of DVH parameters.

Results: For the lung cancer group, differences between the reconstructed mean PTV dose and the values calculated with the TPS were $5.0 \pm 4.2 \%$ (1SD) and $-1.4 \pm 1.5 \%$ for the CA and SA algorithm, respectively. No large differences in the lung and spinal cord DVH parameters were found. For the IMRT treatments, the average dose differences in the PTV were generally below $3 \%$. The reconstructed mean parotid gland dose was $3.2 \pm 1.2 \%$ lower while the maximum spinal cord dose was on average $3.1 \pm 1.9 \%$ higher.

Conclusions: EPID dosimetry combined with 3D dose reconstruction is a useful procedure for patient-specific QA of complex treatments. DVH parameters can be used to interpret the dose distribution delivered to the patient in the same way as during standard treatment plan evaluation.
\end{abstract}




\section{INTRODUCTION}

Patient-specific quality assurance (QA) is an essential step to guarantee accurate patient treatment in radiation therapy. Verification procedures are performed at various levels starting with the QA of machine-related parameters such as beam flatness and stability, accuracy of the leaf positions of a multi-leaf collimator (MLC), and accurate modelling of the linear accelerator at the commissioning stage of the treatment planning system (TPS). Verification procedures for 3D conformal radiation therapy (3D CRT) and intensity-modulated radiotherapy (IMRT) are commonly performed for an individual patient. This last type of verification is the topic of the present paper.

Many institutions perform pre-treatment patient-specific QA by using film measurements in a phantom, specially designed 2D detectors placed on the treatment couch or attached to the treatment head, or Electronic Portal Imaging Devices (EPIDs). ${ }^{1-7}$ In general the energy fluence or the dose in a specific plane is exported from the TPS for a single field or multiple field arrangement and compared to measured data. In case of planar (beam-by-beam) verification, measurements are usually performed using a zero gantry angle. Consequently possible MLC positioning or dose rate differences at other gantry angles are not detected. For beam-by-beam verification it may also be difficult to interpret possible differences in terms of total tumour or normal tissue dose, while composite plan analysis in a phantom has the drawback that the patient geometry and anatomical details are lost.

Therefore various 3D dose reconstruction models ${ }^{8-15}$ have been developed that can reconstruct the $3 \mathrm{D}$ dose distribution inside a phantom or by using a (planning) CT scan, making analysis of a 3D dose distribution more easy. We have developed a pretreatment verification method that is able to reconstruct the 3D dose distribution that will be delivered to a patient and may detect two main sources of error. ${ }^{12,13}$ The first type of error that may be detected is a wrong dose distribution calculated using a too simple dose engine (e.g. a pencil beam algorithm) or a wrong implementation of an algorithm in the TPS. The second main source of error that may be detected is a discrepancy between the actual dose distribution delivered by the linear accelerator, and the planned dose distribution designed by the TPS. This discrepancy can be due to an incorrect dose distribution calculation (e.g. too simple dose engine, wrong implementation of the dose calculation algorithm or incorrect implementation of the linac head model in the TPS) or due to a malfunctioning of the linac itself.

Our dose reconstruction method ${ }^{12,13}$ combines the verification of both sources of error into a total patient-specific QA procedure by making use of an accurate dose calculation algorithm based on Monte Carlo (MC), in combination with the energy fluence of the delivered fields, measured with an EPID. In this paper we will elucidate the application of our method in a clinical setting for two treatment sites irradiated with different techniques: 3D conformal radiation therapy for lung cancers and intensity-modulated radiation therapy for the treatment of head-and-neck cancers. 


\section{MATERIALS AND METHODS}

\section{Patient characteristics and treatment planning procedure}

We analysed two groups of patients planned using our TPS: XiO version 4.2.2 (CMS, St. Louis, USA) for 3D CRT and XiO version 4.3.3 for IMRT.

First, a group of 9 lung cancer patients treated with a 3D conformal technique consisting mostly of 3 beams: a posterior-anterior beam and two lateral beams with 10 MV photons, applying virtual wedges if necessary. These 9 patients were retrospectively selected to assure considerable inter-patient variation in tumour size and location, and are therefore representative for a larger number of clinically applied treatment plans. Gross tumour volume (GTV) is defined using a planning CT-PET scan; the clinical target volume (CTV) is an expansion of the GTV with $5 \mathrm{~mm}$, and a $10 \mathrm{~mm}$ additional margin is applied to create the planning target volume (PTV). Critical structures that are taken into account during treatment planning are the lungs, the spinal cord and if necessary the heart and/or the esophagus. The dose in the PTV is escalated with twice a day $1.8 \mathrm{~Gy}$ fractions, up to a maximum value of $79.2 \mathrm{~Gy}$ or until a mean lung dose (MLD) of 19 Gy or a spinal cord dose of $54 \mathrm{~Gy}$ is reached. ${ }^{16}$ Treatment plan design and dose calculations were performed using the Fast-Fourier Transform convolution algorithm (CA) and were also recalculated for this study using the more advanced Multi-Grid superposition algorithm (SA). ${ }^{17}$ For the recalculation using the SA, all beam parameters including the number of monitor units were identical to that applied using the original CA plan. Both algorithms were implemented in the same version of the TPS (XiO version 4.2.2). The CA is a 'type-a' algorithm according to the classification of Fogliata et al. ${ }^{18}$, whereas the SA is classified as an advanced 'type- $b$ ' model. A grid size of $3 \times 3 \times 3 \mathrm{~mm}^{3}$ was used for all calculations.

The second group of patients consisted of 5 head-and-neck cancer patients treated with a step-and-shoot 7-field IMRT technique applying a $6 \mathrm{MV}$ photon beam with gantry angles of 206, 258, 309, 0, 51, 102 and 154 degrees (IEC convention) and collimator angle increments of 5 degrees from -15 to +15 degrees. An intermediate and high risk PTV, each having a specific dose level, were defined on a planning CT-PET scan as the expansion of the corresponding CTVs with a $5 \mathrm{~mm}$ additional margin. For the intermediate risk PTV a dose of 54 Gy was prescribed, and 68 Gy or 70 Gy for the high risk PTV. Treatment plans for this group were designed solely with the SA algorithm implemented in the TPS (XiO version 4.3.3) using a grid size of $2 \times 2 \times 2 \mathrm{~mm}^{3}$.

\section{D dose reconstruction method}

A 3D dose reconstruction method was previously developed that combines the energy fluence of the beams delivered by the linear accelerator (Siemens Oncor, Siemens Medical Solutions, Concord, CA, USA) measured with an EPID (Siemens OptiVue 500, 1000 or 1000ST), and a dose calculation based on the Monte Carlo dose engine $\mathrm{XVMC}^{19}$. The energy fluence is measured pre-treatment without a phantom or the patient in the beam. The accuracy of this dose reconstruction method was extensively verified using film and ionisation chamber measurements, and differences in absolute 
dose are generally within $3 \%$ compared to measurements for both homogeneous ${ }^{12}$ and inhomogeneous ${ }^{13}$ phantoms.

The treatment beams are delivered and measured with the EPID using a dummy run (one-to-one copy of the clinical plan including all beam, collimator and gantry settings) of the fields prior to patient treatment. The captured EPID images are converted using an in-house developed calibration model into portal dose images. ${ }^{20}$ No additional correction for the use of virtual wedges was applied. For the measured IMRT fields, two additional corrections were performed. First, the ghosting correction was performed per segment instead of a single correction for the entire portal dose image. Second, to compensate for the difference between EPID response for unattenuated energy fluence of the open field and attenuated energy fluence through the MLC and jaws, the raw pixel values outside the field (indicated by the $20 \%$ isodose of the particular segment) were corrected as suggested by Li et al. ${ }^{21}$ From these portal dose images the phase-space is extracted by assuming a point source at the target and a given energy spectrum. The Monte Carlo dose calculation is started using the planning CT scan for each of the beams, which included a conversion factor from dose-to-tissue to dose-to-water for each voxel as described by Siebers et al. ${ }^{22}$ The individual dose distributions are summed and multiplied by the number of fractions. The dose distribution is compared with the planned dose distribution either by looking at isodose surfaces of specific slices or by using a dose-volume histogram (DVH). The Monte Carlo calculation uses a grid size of $3 \times 3 \times 3 \mathrm{~mm}^{3}$ and particles from the phase-space are sampled until a statistical uncertainty in the dose per voxel inside the PTV of approximately $1 \%$ is achieved.

\section{Analysis using DVH parameters}

For the lung cancer patients, differences in dose distribution are analysed using the mean dose, $D_{5 \%}$ and $D_{95 \%}$ ( $D_{x \%}$ is the minimum dose to $x \%$ of the volume) of the PTV. For the normal tissue the mean lung dose, $V_{5}, V_{10}, V_{20}, V_{30}$ and $V_{50}$ are calculated inside the structure defined as the lungs without all GTVs $\left(V_{x}\right.$ is the relative volume receiving more than $x$ Gy). For the spinal cord the maximum dose expressed as $D_{0.1 \%}$ is calculated.

For the head-and-neck cancer patients, differences are analysed using the $D_{5 \%}$, $D_{95 \%}$ and mean dose in the high and intermediate-risk PTVs. For the normal tissues, the parameters that are analysed are the mean dose in the parotid glands and the maximum dose $\left(D_{0.1 \%}\right)$ in the spinal cord.

Differences have been expressed as a mean \pm 1 standard deviation (SD). Relative differences between planned values $D_{\text {xio }}$ and reconstruction dose values $D_{\text {Rec }}$ are expressed as the average of the pair-wise differences according to: $\left(1-D_{\mathrm{XiO}} / D_{\mathrm{Rec}}\right) * 100$.

Although we choose representative cases for each treatment site, we also calculated the statistical significance for groups with more than 5 subjects using a Wilcoxon signed rank test with a Bonferroni correction of the $p$-value by a factor 2 for the multiple comparisons. Statistically significance is assumed for a $p$-value $<0.05$. 


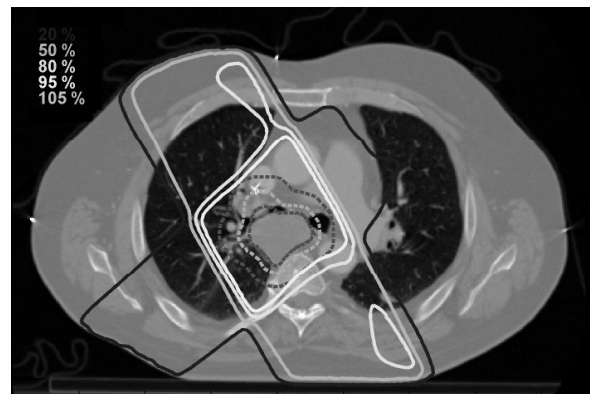

convolution algorithm

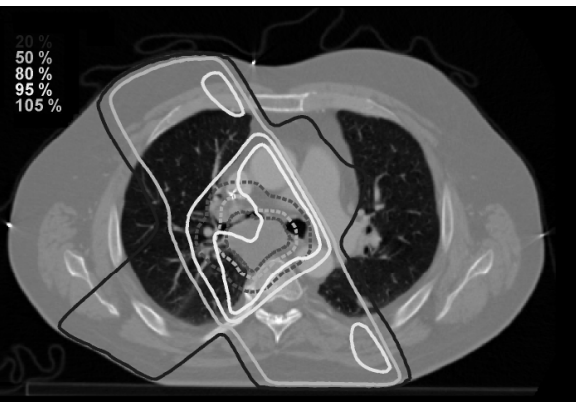

superposition algorithm

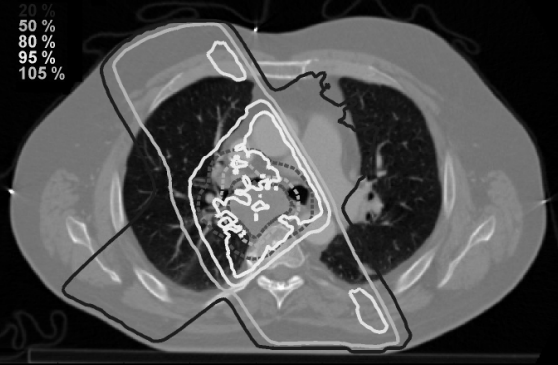

3-D dose reconstruction

Figure 5.1: Dose distributions of a lung cancer treatment obtained by using the convolution or superposition algorithm of the TPS, and the 3D EPID-based reconstructed dose distribution. The GTV, CTV and PTV are shown as dotted lines.

\section{Analysis using overlapping isodose surfaces}

Overlapping isodose surfaces have been used to visually inspect the dose distribution of the dose reconstruction method.

\section{RESULTS}

\section{Lung cancer patient analysis}

For the 9 patients analysed, the size of the PTV ranged from $51 \mathrm{~cm}^{3}$ to $823 \mathrm{~cm}^{3}$ and was located 5 times in the right and 4 times in the left lobe. Four tumours were located peripherally and five centrally. An example of a transversal CT-slice with the calculated dose distribution using either the convolution or the superposition algorithm of the TPS and our 3D dose reconstruction method is shown in Figure 5.1. The intended coverage of the PTV with the $95 \%$ isodose, as designed during the planning process using the CA, is in reality not achieved, as confirmed by recalculating with the SA algorithm, and with our dose reconstruction method. The resulting dose-volume histograms for this patient are shown in Figure 5.2. 


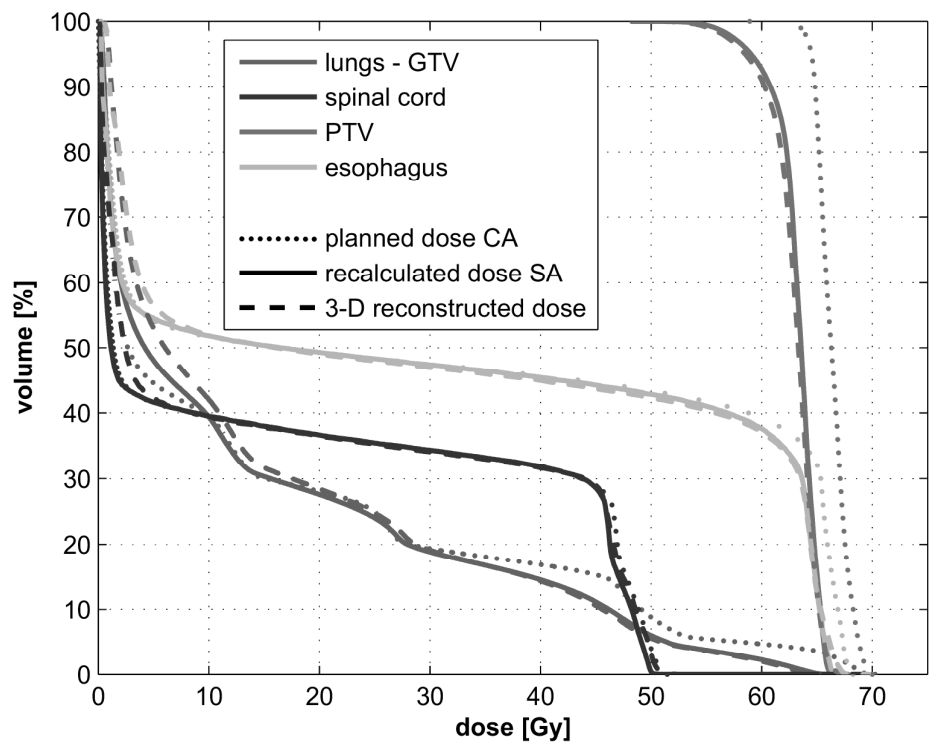

Figure 5.2: Dose-volume histograms for the various dose distributions shown in Figure 5.1.

The results for the DVH parameters of the PTV, lungs and spinal cord are shown in Table 5.1. On average the calculated mean PTV dose using the convolution algorithm $(60.4 \pm 7.6 \mathrm{~Gy})$ or superposition algorithm $(56.7 \pm 6.9 \mathrm{~Gy})$ differed $5.0 \pm 4.2 \%(p=0.022)$ and $-1.4 \pm 1.5 \%$ ( $p=N . S$.$) , respectively, from the mean PTV dose calculated using the$ dose reconstruction method $(57.5 \pm 7.0 \mathrm{~Gy})$. A large difference of $9.2 \pm 7.7 \%(p=0.016)$ was also found between the $D_{95 \%}$ calculated using the convolution algorithm $(58.0 \pm 7.5$ $\mathrm{Gy})$ and dose reconstruction method $53.1 \pm 6.7 \mathrm{~Gy}$. No significant difference $(-0.3 \pm 1.2 \%)$ between SA and dose reconstruction could be observed.

For the spinal cord, only small differences were found in the maximum dose $\left(D_{0.1 \%}\right)$ in the spinal cord for the convolution, superposition algorithm and the dose reconstruction. For the lungs, various parameters are given in Table 5.1 which show deviations between the parameters calculated using the CA and SA dose distributions and the dose reconstruction method.

These differences are also visible in the DVH shown in Figure 5.2. For the low-dose part (below $25 \mathrm{~Gy}$ ) the reconstructed dose distribution is higher compared to the TPS dose calculations. This affects the MLD, $V_{05}, V_{10}, V_{20}$ and $V_{30}$ parameters and results in statistically significant differences $(p=0.016)$ between reconstructed and SA calculated dose distributions. These differences in $V_{05}$ to $V_{30}$ parameters gradually decrease from $9 \%$ to $3 \%$.

\section{Head-and-neck cancer patient analysis}

The head-and-neck IMRT patient group consisted of one nasopharynx, two oropharynx and two hypopharynx cancer patients. The characteristics of the IMRT dose distributions are shown in Table 5.2. An example of the planned and reconstructed dose 
distribution is shown in Figure 5.3, together with the DVH in Figure 5.4. Visual inspection of the two overlapping dose distributions shows similarity between planned and reconstructed dose values both in the high dose as well as in the low dose regions, except in the region of the spinal cord. This difference is also visible in the DVH of the spinal cord in Figure 5.4.

There were only small differences in the dose distributions in the high or intermediate risk PTVs. Also only minor differences were found in the parameters derived from the $\mathrm{DVH}$, such as the $\mathrm{D}_{5 \%}, \mathrm{D}_{95 \%}$ or the mean dose. All average values were generally within 3\% (see Table 5.2).

The reconstructed 3D dose distribution showed a slightly higher dose of $3.1 \pm 1.9 \%$ for the maximum dose $\left(D_{0.1 \%}\right)$ in the spinal cord compared to the TPS dose calculation. For the left and right parotid glands on average a small decrease in the mean dose of $3.0 \pm 0.9 \%$ and $3.3 \pm 1.6 \%$, respectively, was observed for the dose calculated with the SA algorithm relative to the $3 \mathrm{D}$ reconstructed dose.

Table 5.1: Characteristics and dose-volume parameters applied for analysis of the different dose distributions of the lung cancer patients.

\begin{tabular}{|c|c|c|c|c|c|c|c|c|}
\hline \multirow{2}{*}{$\begin{array}{c}\text { Struc- } \\
\text { ture }\end{array}$} & \multirow{2}{*}{$\begin{array}{l}\text { Para- } \\
\text { meter }\end{array}$} & \multirow{2}{*}{$\begin{array}{c}\text { Con- } \\
\text { volution } \\
\text { algo- } \\
\text { rithm } \\
\text { mean } \\
\text { (SD) }\end{array}$} & \multirow{2}{*}{$\begin{array}{c}\text { Super- } \\
\text { position } \\
\text { algo- } \\
\text { rithm } \\
\text { mean } \\
\text { (SD) }\end{array}$} & \multirow{2}{*}{$\begin{array}{c}\text { 3D dose } \\
\text { recon- } \\
\text { struction } \\
\text { mean } \\
\text { (SD) }\end{array}$} & \multicolumn{2}{|c|}{$\begin{array}{l}\text { Convolution } \\
\text { alg. vs. 3D dose } \\
\text { reconstruction }\end{array}$} & \multicolumn{2}{|c|}{$\begin{array}{l}\text { Superposition } \\
\text { alg. vs. 3D dose } \\
\text { reconstruction }\end{array}$} \\
\hline & & & & & $\begin{array}{c}\text { mean } \\
(\mathrm{SD}) \\
{[\%]}\end{array}$ & $\begin{array}{c}\text { p- } \\
\text { value }\end{array}$ & $\begin{array}{c}\text { mean } \\
(\mathrm{SD}) \\
{[\%]}\end{array}$ & $\begin{array}{c}p- \\
\text { value }\end{array}$ \\
\hline \multirow{3}{*}{ PTV } & $\mathrm{D}_{5 \%}(\mathrm{~Gy})$ & $\begin{array}{l}62.9 \\
(7.9)\end{array}$ & $\begin{array}{l}59.7 \\
(7.0)\end{array}$ & $\begin{array}{l}61.1 \\
(7.3)\end{array}$ & $\begin{array}{c}2.9 \\
(2.7)\end{array}$ & 0.042 & $\begin{array}{l}-2.2 \\
(2.0)\end{array}$ & 0.034 \\
\hline & $D_{95 \%}(G y)$ & $\begin{array}{l}58.0 \\
(7.5)\end{array}$ & $\begin{array}{l}53.0 \\
(6.8)\end{array}$ & $\begin{array}{l}53.1 \\
(6.7)\end{array}$ & $\begin{array}{c}9.2 \\
(7.7)\end{array}$ & 0.016 & $\begin{array}{l}-0.3 \\
(1.2)\end{array}$ & N.S. \\
\hline & mean (Gy) & $\begin{array}{l}60.4 \\
(7.6)\end{array}$ & $\begin{array}{l}56.7 \\
(6.9)\end{array}$ & $\begin{array}{l}57.5 \\
(7.0)\end{array}$ & $\begin{array}{c}5.0 \\
(4.2)\end{array}$ & 0.022 & $\begin{array}{l}-1.4 \\
(1.5)\end{array}$ & N.S. \\
\hline $\begin{array}{l}\text { Spinal } \\
\text { Cord }\end{array}$ & $\mathrm{D}_{0.1 \%}(\mathrm{~Gy})$ & $\begin{array}{c}45.0 \\
(10.2)\end{array}$ & $\begin{array}{c}44.8 \\
(10.3)\end{array}$ & $\begin{array}{l}45.3 \\
(9.5)\end{array}$ & $\begin{array}{l}-1.1 \\
(5.0)\end{array}$ & N.S. & $\begin{array}{l}-1.6 \\
(5.9)\end{array}$ & N.S. \\
\hline \multirow{6}{*}{$\begin{array}{l}\text { Lungs } \\
\text { - GTV }\end{array}$} & MLD (Gy) & $\begin{array}{l}12.6 \\
(4.5)\end{array}$ & $\begin{array}{l}11.8 \\
(4.3)\end{array}$ & $\begin{array}{l}12.7 \\
(4.4)\end{array}$ & $\begin{array}{l}-2.0 \\
(3.7)\end{array}$ & N.S. & $\begin{array}{l}-8.3 \\
(2.9)\end{array}$ & 0.016 \\
\hline & $V_{5}(\%)$ & $\begin{array}{c}40.0 \\
(13.4)\end{array}$ & $\begin{array}{c}42.0 \\
(13.6)\end{array}$ & $\begin{array}{c}46.1 \\
(14.4)\end{array}$ & $\begin{array}{r}-14.0 \\
(4.3)\end{array}$ & 0.016 & $\begin{array}{c}-9.3 \\
(2.4)\end{array}$ & 0.016 \\
\hline & $\mathrm{V}_{10}(\%)$ & $\begin{array}{c}34.4 \\
(12.5)\end{array}$ & $\begin{array}{c}33.4 \\
(12.4)\end{array}$ & $\begin{array}{c}36.1 \\
(12.7)\end{array}$ & $\begin{array}{l}-5.4 \\
(5.5)\end{array}$ & 0.042 & $\begin{array}{l}-7.9 \\
(2.9)\end{array}$ & 0.016 \\
\hline & $V_{20}(\%)$ & $\begin{array}{l}21.7 \\
(7.9)\end{array}$ & $\begin{array}{l}21.4 \\
(7.8)\end{array}$ & $\begin{array}{l}22.6 \\
(8.0)\end{array}$ & $\begin{array}{c}-4.1 \\
(3.6)\end{array}$ & 0.022 & $\begin{array}{c}-5.4 \\
(3.1)\end{array}$ & 0.016 \\
\hline & $V_{30}(\%)$ & $\begin{array}{l}17.7 \\
(6.9)\end{array}$ & $\begin{array}{l}16.6 \\
(6.8)\end{array}$ & $\begin{array}{l}17.0 \\
(6.9)\end{array}$ & $\begin{array}{c}4.7 \\
(4.5)\end{array}$ & 0.022 & $\begin{array}{c}-2.7 \\
(2.0)\end{array}$ & 0.016 \\
\hline & $V_{50}(\%)$ & $\begin{array}{c}8.6 \\
(5.7)\end{array}$ & $\begin{array}{c}6.1 \\
(5.1)\end{array}$ & $\begin{array}{c}6.3 \\
(5.2)\end{array}$ & $\begin{array}{c}58.0 \\
(58.4)\end{array}$ & 0.024 & $\begin{array}{c}-4.8 \\
(10.7)\end{array}$ & N.S. \\
\hline
\end{tabular}

N.S. = not significant. Note: The large standard deviations (SD) in the dose-volume parameters are the result of inter patient variability due to the dose escalation protocol that was used. 


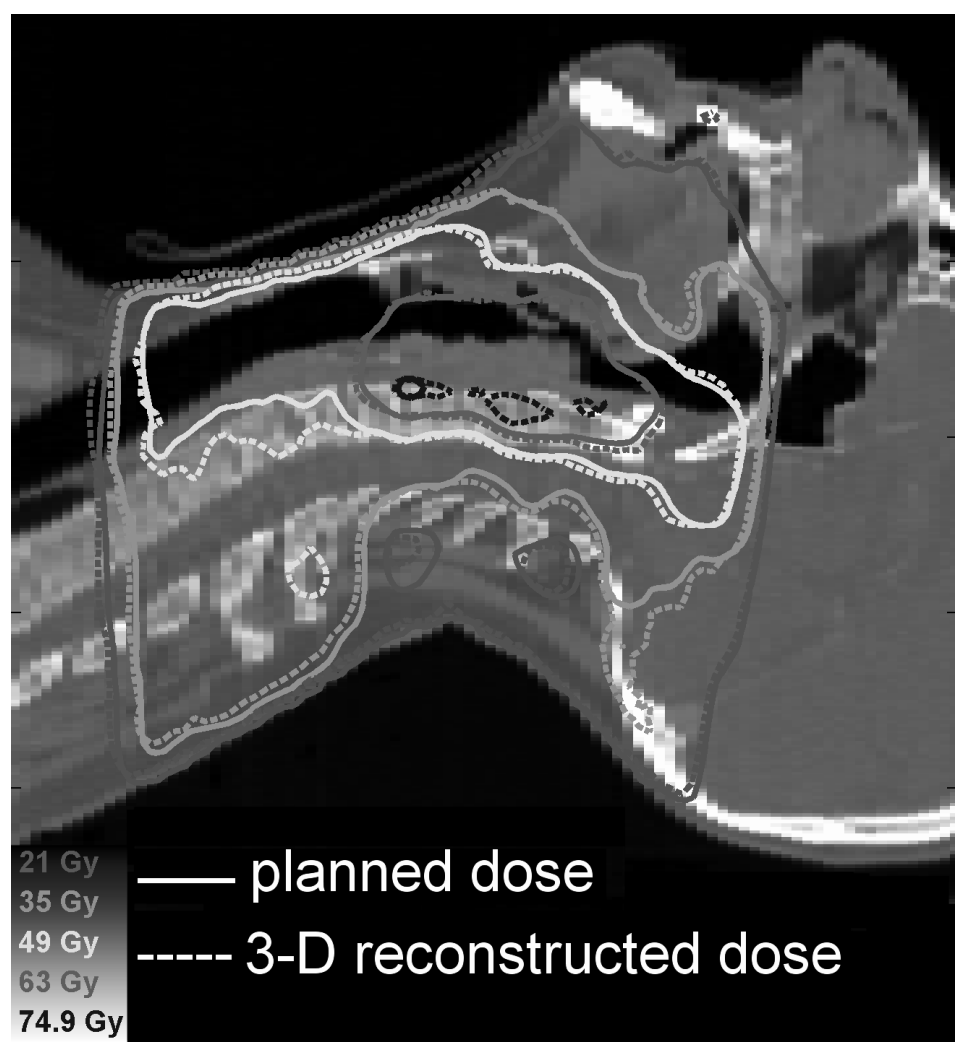

Figure 5.3: Example of a head-and-neck IMRT plan. The solid lines represent the calculated dose distribution using the superposition algorithm of the TPS and the dashed lines represent the reconstructed $3 D$ dose distribution.

Table 5.2: Characteristics and dose-volume parameters of the head-and-neck cancer patients.

\begin{tabular}{|c|c|c|c|c|}
\hline Structure & Parameter & $\begin{array}{c}\text { Superposition } \\
\text { algorithm } \\
\text { mean (SD) } \\
\text { [Gy] }\end{array}$ & $\begin{array}{c}\text { 3D dose } \\
\text { reconstruction } \\
\text { mean (SD) } \\
\text { [Gy] }\end{array}$ & $\begin{array}{c}\text { Relative } \\
\text { difference } \\
\text { mean (SD } \\
\text { [\%] }\end{array}$ \\
\hline $\begin{array}{l}\text { PTV } \\
\text { high risk }\end{array}$ & $\begin{array}{l}D_{5 \%} \\
\text { mean } \\
D_{95 \%}\end{array}$ & $\begin{array}{l}72.9(2.0) \\
69.6(1.0) \\
66.4(0.6)\end{array}$ & $\begin{array}{l}72.6(1.9) \\
69.1(1.1) \\
64.9(1.5)\end{array}$ & $\begin{array}{l}-0.4(1.2) \\
-0.7(0.8) \\
-2.3(1.6)\end{array}$ \\
\hline $\begin{array}{l}\text { PTV } \\
\text { intermediate } \\
\text { risk }\end{array}$ & $\begin{array}{l}\mathrm{D}_{5 \%} \\
\text { mean } \\
\mathrm{D}_{95 \%}\end{array}$ & $\begin{array}{l}68.7(6.4) \\
59.9(3.3) \\
52.6(0.5)\end{array}$ & $\begin{array}{l}68.4(6.7) \\
59.5(3.6) \\
52.1(0.7)\end{array}$ & $\begin{array}{l}-0.5(1.1) \\
-0.7(1.0) \\
-1.0(1.6)\end{array}$ \\
\hline $\begin{array}{l}\text { Spinal Cord } \\
\text { Left Parotid } \\
\text { Right Parotid }\end{array}$ & $\begin{array}{l}\mathrm{D}_{0.1 \%} \\
\text { mean } \\
\text { mean }\end{array}$ & $\begin{array}{l}45.2(1.4) \\
48.3(7.0) \\
50.5(8.8)\end{array}$ & $\begin{array}{l}46.7(1.5) \\
46.9(6.9) \\
49.0(9.2)\end{array}$ & $\begin{array}{l}3.1(1.9) \\
-3.0(0.9) \\
-3.3(1.6)\end{array}$ \\
\hline
\end{tabular}




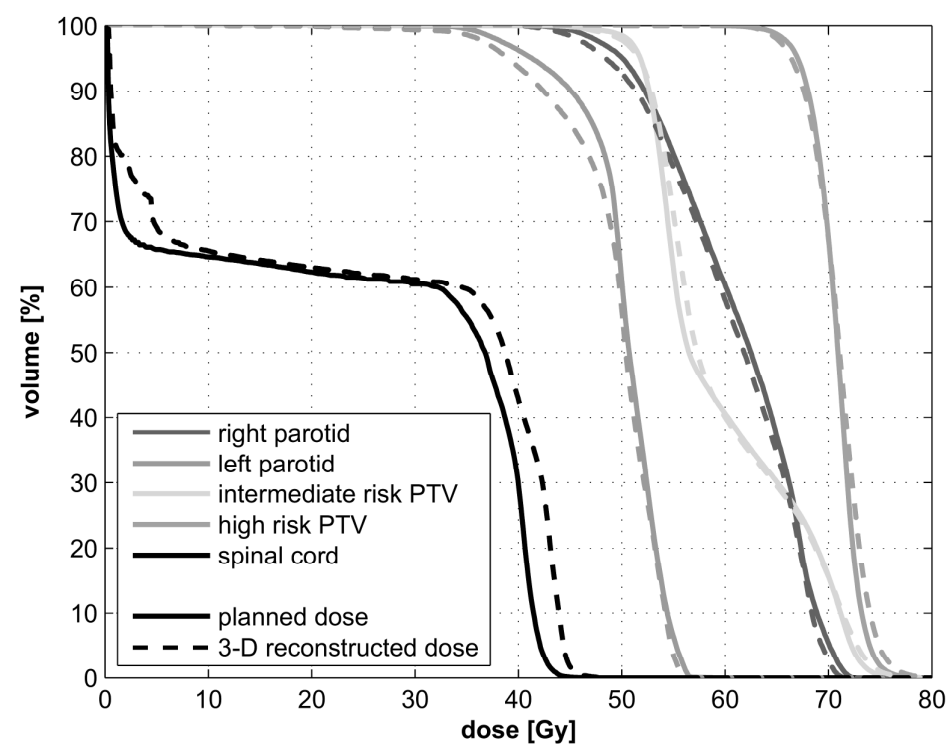

Figure 5.4: Example of a dose-volume histogram of the planned and reconstructed dose distribution for the PTV and normal tissues for the patient shown in Figure 5.3.

\section{DISCUSSION}

Patient-specific QA is performed in most institutions for the verification of complex (IMRT) plans before the start of treatment. Many institutions perform ionisation chamber and film dosimetry inside a phantom and compare the results with dose distributions calculated by the TPS for this situation. In such a way the actual delivery of the planned treatment beams is verified and also, to a certain degree, the dose calculation of the TPS. However, if differences are observed with such a 'hybrid' approach, it is not always easy to understand how these differences will affect the dose distribution for a specific patient geometry.

With our 3D dose reconstruction method differences can be quantified in terms of dose in the PTV or dose in the normal tissues. This will ease the interpretation of a 3D reconstructed dose distribution of a treatment plan evaluation based on the objectives that are set for this treatment plan. For instance for the lung cancer patient group, coverage of the PTV with the $95 \%$ isodose surface must be achieved, the maximum dose in the spinal cord may not exceed $54 \mathrm{~Gy}$ or the $\mathrm{V}_{20}$ for the lungs must be smaller than $30 \%$, which can easily be checked by looking at the dose-volume histogram.

Because our dose reconstruction method uses a Monte Carlo-based dose calculation engine, it is likely that there will be differences between simple algorithms such as the convolution algorithm in regions with high gradients in density. This is clearly shown in the reconstruction of the dose in the PTV for lung tumours that are located 
inside the low-density lung tissue. The largest reduction in mean PTV dose for a specific lung cancer patient in this study was as high as $14 \%$. These differences could, however, also be picked up by a recalculation of the dose distribution using a more advanced algorithm. In our department, we have switched recently from the simple convolution algorithm to the more advanced superposition algorithm for all dose calculations and each treatment site.

For the normal tissue in the lung cancer group there are some differences visible in the low-dose region indicated by the $V_{5}, V_{10}$ and $V_{20}$ ranging from $5 \%$ to $10 \%$, which are also visible in the DVH in Figure 5.2. Two factors contribute to this difference. The first is that the treatment head of the Siemens linac is not modelled properly in the XiOTPS. The back-up jaws are not present in the current Siemens MLC design but are modelled in the XiO-TPS version 4.2.2, which results in a lower planned dose behind closed leaves than actually delivered. The advantage of the presented dose reconstruction method is that it is based on the actually measured energy fluence and inherently takes differences between planned and delivered energy fluence through the MLC into account. The second factor that is likely to contribute to the difference is the fact that only a single photon energy spectrum is used in the dose reconstruction method. Including an additional photon energy spectrum with a higher mean energy for photons transmitted through the MLC and collimator jaws may improve the accuracy in the dose reconstruction in these low-dose regions.

For the head-and-neck IMRT cancer patients, differences in the PTV are very small, around $1 \%-2 \%$, indicating a high accuracy in the dose calculation and dose reconstruction in the high-dose regions. For the low-dose regions, for example in the spinal cord, the reconstructed dose might be due overestimated due to the difference in photon energy spectrum of the large portion of fluence that is delivered through the MLC for highly modulated IMRT treatments.

In our method the starting point of the dose calculation is based on the measured energy fluence of the actually delivered fields prior to treatment. With this approach possible differences between planned and delivered beam characteristics can be detected. However, what is more important is that these differences can directly be visualised in the planning CT scan. For example, small differences in leaf-position have a negative impact on the delivered dose distribution but it is also possible that these differences cancel out. Furthermore, previous studies showed that possible errors in the TPS such as wrong tongue-and-groove parameters or block transmission values may be detected and characterised in the patient geometry. ${ }^{13,15,23}$

An important issue for patient-specific QA of treatment plans and dose distributions is the time such a verification takes. The presented verification procedures can be automated in such a way the only time required to start the verification is the delivery of the treatment fields in a dummy run outside patient treatment time. Dose reconstruction can start automatically after the delivery of the beams and the time to finish a dose reconstruction depends on the number of delivered fields and the statistical accuracy of the Monte Carlo calculation. For a typical 7-field IMRT head-and-neck cancer case with a $1 \%$ statistical uncertainty takes approximately 6 hours on a dualprocessor $1.8 \mathrm{GHz} \mathrm{CPU}$, a lower statistical uncertainty of $2 \%$ takes only $90 \mathrm{~min}$ for this configuration. 
In our department, the presented dose reconstruction method is currently only used on demand for the verification of complex (IMRT) cases. We will implement our approach on a routine basis for all our IMRT patients in the near future to replace phantom dosimetry with the ionisation chamber and film dosimetry by EPID dosimetry combined with 3D dose reconstruction.

\section{CONCLUSIONS}

In this work the clinical implementation was shown of a patient-specific dose verification method that reconstructs the dose distribution based on EPID dosimetry in combination with a planning CT scan. The dose calculation engine is independent of the TPS and uses a Monte Carlo computation. Differences, either due to the type of dose calculation algorithm, dose calculation errors of the TPS or due to machine related parameters, can be quantified and analysed in terms of differences in dose in the PTV or normal tissues. The analysis can be performed either on a slice-to-slice basis using overlapping isodose surfaces or by looking at the dose-volume histograms. Our method is an attractive alternative for patient-specific pre-treatment (both 3D CRT and IMRT) verification using ionisation chamber and film dosimetry.

\section{REFERENCES}

${ }^{1}$ B. Warkentin, S. Steciw, S. Rathee, and B.G. Fallone, "Dosimetric IMRT verification with a flatpanel EPID," Med Phys 30, 3143-3155 (2003).

${ }^{2}$ W.J.C. van Elmpt, S.M.J.J.G. Nijsten, B.J. Mijnheer, and A.W.H. Minken, "Experimental verification of a portal dose prediction model," Med Phys 32, 2805-2818 (2005).

${ }^{3}$ S. Gillis, C. De Wagter, J. Bohsung, B. Perrin, P. Williams, and B.J. Mijnheer, "An inter-centre quality assurance network for IMRT verification: results of the ESTRO QUASIMODO project," Radiother Oncol 76, 340-353 (2005).

${ }^{4}$ A. Van Esch, T. Depuydt, and D.P. Huyskens, "The use of an aSi-based EPID for routine absolute dosimetric pre-treatment verification of dynamic IMRT fields," Radiother Oncol 71, 223 234 (2004).

${ }^{5}$ M. Wendling, R.J. Louwe, L.N. McDermott, J.J. Sonke, M. van Herk, and B.J. Mijnheer, "Accurate two-dimensional IMRT verification using a back-projection EPID dosimetry method," Med Phys 33, 259-273 (2006).

${ }^{6}$ S.M. Nijsten, B.J. Mijnheer, A.L. Dekker, P. Lambin, and A.W. Minken, "Routine individualised patient dosimetry using electronic portal imaging devices," Radiother Oncol 83, 65-75 (2007).

${ }^{7}$ M. van Zijtveld, M.L. Dirkx, H.C. de Boer, and B.J. Heijmen, "Dosimetric pre-treatment verification of IMRT using an EPID; clinical experience," Radiother Oncol 81, 168-175 (2006).

${ }^{8}$ J.M. Kapatoes, G.H. Olivera, J.P. Balog, H. Keller, P.J. Reckwerdt, and T.R. Mackie, "On the accuracy and effectiveness of dose reconstruction for tomotherapy," Phys Med Biol 46, 943-966 (2001).

${ }^{9}$ R.J.W. Louwe, E.M.F. Damen, M. van Herk, A.W.H. Minken, O. Torzsok, and B.J. Mijnheer, "Three-dimensional dose reconstruction of breast cancer treatment using portal imaging," Med Phys 30, 2376-2389 (2003). 
${ }^{10}$ W.D. Renner, M. Sarfaraz, M.A. Earl, and C.X. Yu, "A dose delivery verification method for conventional and intensity-modulated radiation therapy using measured field fluence distributions," Med Phys 30, 2996-3005 (2003).

${ }^{11}$ S. Steciw, B. Warkentin, S. Rathee, and B.G. Fallone, "Three-dimensional IMRT verification with a flat-panel EPID," Med Phys 32, 600-612 (2005).

${ }^{12}$ W.J.C. van Elmpt, S.M.J.J.G. Nijsten, R.F.H. Schiffeleers, A.L.A.J. Dekker, B.J. Mijnheer, P. Lambin, and A.W.H. Minken, "A Monte Carlo based three-dimensional dose reconstruction method derived from portal dose images," Med Phys 33, 2426-2434 (2006).

${ }^{13}$ W.J.C. van Elmpt, S.M.J.J.G. Nijsten, A.L.A.J. Dekker, B.J. Mijnheer, and P. Lambin, "Treatment verification in the presence of inhomogeneities using EPID-based three-dimensional dose reconstruction," Med Phys 34, 2816-2826 (2007).

${ }^{14} \mathrm{G}$. Jarry and F. Verhaegen, "Patient-specific dosimetry of conventional and intensity modulated radiation therapy using a novel full Monte Carlo phase space reconstruction method from electronic portal images," Phys Med Biol 52, 2277-2299 (2007).

${ }^{15} \mathrm{M}$. van Zijtveld, M.L. Dirkx, H.C. de Boer, and B.J. Heijmen, "3D dose reconstruction for clinical evaluation of IMRT pretreatment verification with an EPID," Radiother Oncol 82, 201-207 (2007).

${ }^{16}$ A. van Baardwijk, G. Bosmans, L. Boersma, S. Wanders, A. Dekker, A. Dingemans, G. Bootsma, W. Geraedts, C. Pitz, J. Simons, P. Lambin, and D. De Ruysscher, "Individualized radical radiotherapy of non-small cell lung cancer (NSCLC) based on normal tissue dose constraints: a feasibility study.," Int J Radiat Oncol Biol Phys (submitted), (2007).

${ }^{17}$ M. Miften, M. Wiesmeyer, S. Monthofer, and K. Krippner, "Implementation of FFT convolution and multigrid superposition models in the FOCUS RTP system," Phys Med Biol 45, 817-833 (2000).

${ }^{18}$ A. Fogliata, E. Vanetti, D. Albers, C. Brink, A. Clivio, T. Knoos, G. Nicolini, and L. Cozzi, "On the dosimetric behaviour of photon dose calculation algorithms in the presence of simple geometric heterogeneities: comparison with Monte Carlo calculations," Phys Med Biol 52, 1363-1385 (2007).

${ }^{19} \mathrm{M}$. Fippel, "Fast Monte Carlo dose calculation for photon beams based on the VMC electron algorithm," Med Phys 26, 1466-1475 (1999).

${ }^{20}$ S.M.J.J.G. Nijsten, W.J.C. van Elmpt, M. Jacobs, B.J. Mijnheer, A.L.A.J. Dekker, P. Lambin, and A.W.H. Minken, "A global correction model of a-Si EPIDs used for transit dosimetry," Med Phys 34, 3872-3884 (2007).

${ }^{21}$ W. Li, J.V. Siebers, and J.A. Moore, "Using fluence separation to account for energy spectra dependence in computing dosimetric a-Si EPID images for IMRT fields," Med Phys 33, 4468-4480 (2006).

${ }^{22}$ J.V. Siebers, P.J. Keall, A.E. Nahum, and R. Mohan, "Converting absorbed dose to medium to absorbed dose to water for Monte Carlo based photon beam dose calculations," Phys Med Biol 45, 983-995 (2000).

${ }^{23}$ L.N. McDermott, M. Wendling, B. van Asselen, J. Stroom, J.J. Sonke, M. van Herk, and B.J. Mijnheer, "Clinical experience with EPID dosimetry for prostate IMRT pre-treatment dose verification," Med Phys 33, 3921-3930 (2006). 



\section{CHAPTER}

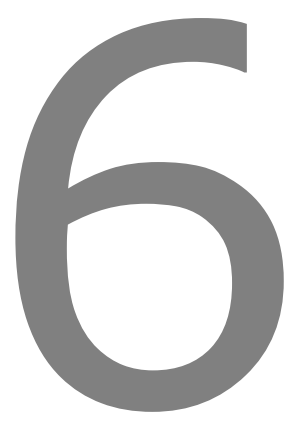

Transition from a simple to a more advanced dose calculation algorithm for radiotherapy of non-small cell lung cancer (NSCLC): Implications for clinical implementation in an individualized dose-escalation protocol

Wouter van Elmpt, Michel Öllers, Marije Velders, Kim Poels, Ben Mijnheer, Dirk De Ruysscher, André Dekker, Philippe Lambin and Liesbeth Boersma

Published in: Radiotherapy and Oncology 88(3):326-334, 2008. 


\begin{abstract}
Background and purpose: To investigate the clinical consequences of the transition from a simple convolution algorithm (CA) to a more advanced superposition dose calculation algorithm (SA) in an individualized isotoxic dose-escalation protocol for NSCLC patients.

Material and methods: First, treatment plans designed according to ICRU50criteria using the CA were recalculated using the SA, for 16 patients. Next, two additional plans were designed for each patient using only the SA: one with 95\%-isodose coverage (ICRU50-criteria), the other allowing PTV coverage with 90\%-isodose at the lung side. PTV dose was escalated to a maximum dose of $79.2 \mathrm{~Gy}$ or lower when limited by either a mean lung dose (MLD) of 19 Gy or a maximum spinal cord dose of $54 \mathrm{~Gy}$. Equivalent uniform doses (EUD) in the PTV were compared.

Results: Recalculation of the CA plans using the SA, showed PTV underdosage in the CA plans: the median PTV EUD was 61.3 Gy (range $44.9-80.4 \mathrm{~Gy}$ ) and $55.5 \mathrm{~Gy}$ $(43.9-76.8 \mathrm{~Gy})$, for CA and SA, respectively $(p<0.001)$. Redesigning plans using the SA resulted in an almost identical PTV EUD of $55.1 \mathrm{~Gy}(43.7-79.2 \mathrm{~Gy})$. For the subgroup $(\mathrm{N}=9)$ with MLD as dose-limiting factor a gain in PTV EUD of $2.7 \pm 1.8 \mathrm{~Gy}(\mathrm{p}=0.008)$ was achieved using the $90 \%$-isodose coverage plan.

Conclusions: Plans calculated using the CA caused large PTV underdosage. Plans designed using the SA often lead to lower maximum achievable tumour doses due to higher MLD values. Allowing somewhat relaxed PTV coverage criteria increased the PTV dose again for MLD restricted cases. Consequently, in clinics where isotoxic individual dose-escalation is applied, implementation of an SA should be accompanied by accepting limited PTV underdosage in patients with MLD as the dose-limiting factor.
\end{abstract}




\section{INTRODUCTION}

It is well known that more advanced algorithms such as the collapsed cone or the superposition algorithm (SA) give a more reliable prediction of the radiation dose in (lung) tissue than more simple algorithms like the pencil beam or convolution algorithm (CA), or by using an equivalent path length (EPL) correction. ${ }^{1-10}$ The main problem with the more simple algorithms is that they do not accurately model the secondary electron transport and energy deposition in regions with large differences in tissue densities, for example lung tissue in the thorax region. This results in an overestimation of absorbed dose and an underestimation of the penumbra width in these low-density regions for these more simple algorithms. De Jaeger et al. ${ }^{4}$ showed that a simple tissue inhomogeneity-correction algorithm like the EPL overestimates the dose in normal lung tissue. Since parts of lung tissue are usually included in the planning target volume (PTV), this means that simple algorithms also overestimate the dose in parts of the PTV. Consequently, treatment plans based on simple algorithms, may in reality result in important underdosage in the PTV. Scholz et al. ${ }^{11}$ showed for example that if the superposition algorithm is used instead of a simple pencil beam algorithm, the median CTV dose decreases by about $4.5 \%$, whereas the median PTV dose even decreases by about $12.1 \%$, while the fraction of the PTV covered by the $90 \%$ isodose was reduced from $95 \%$ to $28.6 \%$.

Since the SA is capable of a more reliable prediction of radiation dose ${ }^{6-10}$, it will allow designing treatment plans that yield good PTV coverage, i.e. coverage of the PTV by the $95 \%$ isodose. However, due to the fact that the actual penumbra is much broader than the penumbra calculated with the simple algorithms, these plans are expected to have larger fields and thus also to yield a higher dose in normal lung tissue. Consequently, implementation of the newer algorithms into clinical practice using the ICRU50-guidelines ${ }^{12}$ for PTV dose inhomogeneities (i.e. the dose in the PTV should be between $95 \%$ and $107 \%$ ) will yield a more realistic coverage ${ }^{1-10}$, but are also expected to result in a higher dose to normal lung tissue. If a fixed radiation dose to the tumour is used, introduction of the newer algorithms will thus lead to more pulmonary toxicity. In our institute however, an individual dose-escalation strategy is applied, similar to several phase I dose-escalation studies where the maximum prescribed dose is based on normal tissue constraints. ${ }^{13-19}$

Transition from the simple to the more advanced algorithms may also have severe consequences for the probability of tumour control. If we assume a patient, in whom the treatment plan is calculated using the simple CA, with a prescribed tumour dose of $70 \mathrm{~Gy}$ at an MLD of $19 \mathrm{~Gy}$, re-calculation of this plan using the more advanced SA algorithm may show that the PTV is only covered by the $70 \%$ isodose, i.e. the actual delivered minimum dose in the PTV was $49 \mathrm{~Gy}$. If we now redesign the treatment plan using the more advanced SA, aiming at covering the PTV with the $95 \%$ isodose surface, and compare the expected tumour control with the CA plan, three categories of scenarios may arise:

1) one in which the SA plan leads to the plan with the highest expected tumour control, 
2) one in which the CA plan leads to the plan with a highest expected tumour control, and

3) one scenario in which it is not clear which plan will lead to the highest tumour control.

Scenario 1 would occur if the SA plan leads to e.g. a maximum prescribed dose at an MLD of $19 \mathrm{~Gy}$ of $70 \mathrm{~Gy}$. In this scenario, the minimum dose in the PTV with the SA is much higher than with the more simple CA ( $95 \%$ of $70 \mathrm{~Gy}$ vs. $70 \%$ of $70 \mathrm{~Gy}$ ). Scenario 2 would occur if the SA plan leads to e.g. maximum prescribed dose at an MLD of $19 \mathrm{~Gy}$ of $45 \mathrm{~Gy}$. In this case, the more advanced redesigned SA plan is much worse and has even a lower tumour control than the original CA plan recalculated with the SA, since the minimum dose of the CA based plan is still higher than the prescribed dose of the SA based plan ( 49 Gy vs. $45 \mathrm{~Gy}$ ). Finally, scenario 3 would occur if the SA plan leads to e.g. a maximum prescribed dose at an MLD of $19 \mathrm{~Gy}$ of $60 \mathrm{~Gy}$. In this case the plan with the simple algorithm has a higher prescribed dose than the SA plan (70 Gy vs. $60 \mathrm{~Gy}$ ), but the minimum dose in the PTV is much lower in the CA plan compared with the SA plan (49 Gy vs. $57 \mathrm{~Gy}$ ).

Although numerous papers ${ }^{1-7}$ described the differences between simple and more advanced dose calculation algorithms, there are no clear guidelines on how they should be applied in clinical routine. Recently, both Morgan et al. ${ }^{6}$ and Bragg et al. ${ }^{5}$ presented detailed comparisons between simple and more advanced algorithms for thoracic treatments. Both papers concluded that the clinical consequences for treatment planning had to be studied in more detail before the transition from simple to more advanced algorithms. Therefore, the purpose of this study is to propose a guideline for such a transition for clinics that use protocols using individual dose-escalation based on normal tissue constraints. In this paper we present a detailed comparison between CA and SA based treatment plans in a planning study of 16 non-small cell lung cancer (NSCLC) patients. To verify the accuracy of the dose calculation of both treatment planning system (TPS) algorithms and the beam modelling in the TPS, an independent dose calculation is performed using an in-house developed dose verification procedure based on Monte Carlo (MC) simulations.

\section{MATERIALS AND METHODS}

\section{Patients and characteristics}

We used the planning PET-CT thorax scans of 16 patients that were treated for lung cancer in our institute in 2005. The clinical TNM stage varied from T1N0 to T4N3M1. Six out of the sixteen patients had N0, seven had N2, and three patients had N3 disease. For T1, T2, T3 and T4 disease these numbers were 1, 6, 2 and 7 patients, respectively.

In all patients the Gross Tumour Volume (GTV) of the primary tumour and the nodes was delineated based on PET-CT data. The Clinical Target Volume (CTV) was defined as the GTV $+0.5 \mathrm{~cm}$ margin. The Planning Target Volume (PTV) was the CTV + $1.0 \mathrm{~cm}$ for the primary lung tumours, and $\mathrm{CTV}+0.5 \mathrm{~cm}$ for the lymph nodes. For 
analysis of dose-volume parameters a PTV was used that is clipped $0.5 \mathrm{~cm}$ beneath the skin.

\section{Radiotherapy treatment planning and dose calculation algorithms}

CT scan based treatment plans were designed using the Fast Fourier Transform convolution algorithm (CA) and the Multi-Grid superposition algorithm (SA) of our clinically applied treatment planning system (XiO, CMS, St. Louis, MO, USA). ${ }^{20}$ The CA and SA are type $a$ and type $b$ algorithms, respectively, according to the classification of Knöös et al. $^{8}$

The convolution algorithm (CA) calculates the photon energy fluence distribution by taking into account the attenuation based on the electron density of the traversed path inside the patient CT scan. Afterwards this photon energy fluence at each point inside the patient is convolved with a primary and a scatter dose deposition kernel to calculate the 3D dose deposition of the beam. The superposition algorithm (SA) applies the same first steps as the convolution algorithm, but the energy deposition kernels are modified to take into account the radiological path length. In this way, low densities (typically in lung tissue) are taken into account by a scaling of the energy deposition kernels. ${ }^{20}$

Both plans were calculated using heterogeneity corrections on a dose grid of $5 \times 5 \times 5$ $\mathrm{mm}^{3}$. Our 3D conformal treatment plans typically consist of 3 treatment beams of 10 MV photons with either a block or the multi-leaf collimator conformed to the PTV and, if necessary, wedges are applied. The objective of the treatment planning procedure was to achieve dose conformance around the PTV and minimize dose in the critical structures. Constraints for the critical structures taken into account for this study are a maximum dose in the spinal cord of 54 Gy and a mean lung dose (MLD) less than 19 Gy. The MLD was defined as the mean dose in both lungs with the GTV excluded. ${ }^{21}$ Taking these constraints into account, the dose inside the PTV was escalated as high as possible up to a maximum prescribed dose of $79.2 \mathrm{~Gy}$ at $1.8 \mathrm{~Gy}$ per fraction twice a day. ${ }^{22}$ The maximum of 79.2 Gy was chosen based on the results of Marks et al. ${ }^{23}$, who defined the maximum tolerated dose for Grade $\geq$ III pulmonary toxicity to be at about $80 \mathrm{~Gy} / 5$ weeks.

To investigate the impact of using the new SA algorithm, the first step was to make an estimation of the dose distributions that had actually been delivered, by recalculating the treatment plans designed using the convolution algorithm (plan 1-CA) with the more advanced superposition algorithm. For this purpose, treatment plans calculated with the CA were recalculated using the new SA, using the same number of monitor units (plan 1-SA). To quantify the differences between the dose distributions, MLD, maximum spinal cord dose, the dose at the prescription point and the mean dose in the PTV were calculated. In addition, the equivalent uniform dose (EUD) was calculated in the PTV (see below).

In the second step, new treatment plans were designed and calculated using the SA, based on the same criteria, and equivalent constraints as applied for the CA plan, as recalculated by the 1-SA plan (see below in results section), i.e. PTV coverage between $95-107 \%$, MLD <18.1 Gy, spinal cord dose <54 Gy, and maximum prescribed dose $<76.5$ Gy (plan 2-SA). 
Since we anticipated that using the same PTV constraints in the SA plan would yield much larger irradiation fields, having a much higher MLD and consequently a lower prescribed dose, a third plan was designed using the SA (plan 3-SA). For this plan, the criteria for the PTV at the lung side were relaxed to a coverage by the $90 \%$ isodose line, thereby allowing an increase in maximum achievable PTV dose.

\section{Parameters to evaluate the dose distributions of the different treatment plans}

As explained above, the prescribed dose was escalated up to maximum value of 79.2 Gy (or 76.5 Gy for the 2-SA and 3-SA plans), until the MLD exceeded 19 Gy (or $18.1 \mathrm{~Gy}$ for the 2-SA and 3-SA plans), or until the maximum spinal cord dose exceeded $54 \mathrm{~Gy}$. Obviously, this strategy was chosen to maximize the tumour control probability (TCP) for the individual patient, with an acceptable risk on normal tissue complications. ${ }^{22}$ However, quantification of the TCP based on the 3D dose distributions is difficult and depends largely on the specific biological parameters chosen. Therefore, we analyzed several surrogate parameters related to the TCP. First of all, we evaluated the prescribed PTV dose and the mean PTV dose. However, in case of extreme doseinhomogeneity (PTV doses $<90 \%$, see discussion), the mean dose is not a good parameter to estimate the TCP. ${ }^{24}$ Therefore, also the equivalent uniform dose (EUD) within the PTV was calculated. ${ }^{25}$ The EUD is defined as the dose needed to obtain the same survival fraction as the inhomogeneous dose distribution $D_{i}$ and can be calculated from the dose-volume histogram (DVH). For the tumour, the EUD is calculated using the differential dose-volume histogram $v_{i}$ of the PTV:

$$
E U D=D_{\text {ref }} \ln \left(\sum_{i} v_{i}\left(S F_{\text {ref }}\right)^{D_{i} / D_{\text {ref }}}\right) / \ln \left(S F_{\text {ref }}\right) \text {, }
$$

with $\mathrm{SF}_{\text {ref }}$, the surviving fraction at the reference dose $\mathrm{D}_{\text {ref }}$ of $1.8 \mathrm{~Gy}$ per fraction equal to 0.5 , which results from an $\alpha$-value of $0.4 .^{25}$

\section{Comparison of treatment plans and statistical analysis}

Ranking of the treatment plans was performed based on the maximum EUD that could be reached taken into account the normal tissue constraints. Also scored was the number of times a particular constraint was reached, i.e. MLD, spinal cord or maximum prescribed dose.

Furthermore, the relation between the mean dose in the PTV and the EUD was investigated as well as the influence of the dose calculation algorithm and tumour volume or location inside the thorax on the final ranking.

Statistically significant differences were evaluated using a Wilcoxon signed rank test (SPSS, version 12.0.1, Chicago, USA) and statistically significance was assumed for $p$-values smaller than 0.05 . No statistical differences ( $p$-value) were calculated for groups with 5 or less subjects. 


\section{Dose verification}

Verification of the calculated dose distributions of the TPS was performed using a 3D dose reconstruction method using the actual delivered beams measured using an amorphous silicon electronic portal imaging device (EPID) as the input for a dose calculation algorithm based on the Monte Carlo (MC) code XVMC. ${ }^{26}$ In this method the dose delivered by the linac is measured using the EPID calibrated for portal dosimetry, and reconstructs the dose distribution applying the planning CT scan. The method has been validated with phantom measurements demonstrating a high accuracy for both homogeneous and inhomogeneous geometries. ${ }^{10,27}$ Such an approach of dose verification is totally independent of the TPS detecting both possible errors in implementation (beam commissioning) of the linac beams in the TPS and limitations of the dose calculation algorithm. This verification was performed using a subset $(\mathrm{N}=5)$ of the patients that were evaluated by reconstructing the dose distribution of plan 1 . These patients were selected based on having a large variety in tumour size and location inside the thorax. Recalculated MC dose distributions are indicated with the suffix MC, e.g. plan 1-MC. MC calculations are in principle the best estimate that is available to calculate the dose delivered to the patient. Differences in MLD, EUD and maximum spinal cord dose between CA, SA and $M C$ are assessed to investigate the overall accuracy of the TPS. For the comparison with the MC calculations the $D_{0.1 \%}$ value (i.e. the dose received by at least $0.1 \%$ of the spinal cord) is used due to the presence of statistical noise in the $\mathrm{MC}$ dose distribution.

\section{RESULTS}

\section{Comparison between different dose calculation algorithms}

\section{Comparison of plan 1-CA with plan 1-SA}

The first step was to recalculate with the SA the treatment plans that had been designed and calculated with the CA, applying the same number of monitor units (plan 1SA). Analysis of the PTV dose showed that the prescribed dose that has been delivered clinically in the years we designed treatment plans using the CA, 61.0 Gy (range 44.6 $79.2 \mathrm{~Gy})$, has actually on average been $3.3 \pm 2.9 \%$ (1 SD) $(p<0.001)$ lower, as estimated by the SA, 59.4 Gy $(43.7-76.0 \mathrm{~Gy})$.

In addition, the PTV coverage appeared to be very poor: underdosages up to $30 \%$ (e.g. patient 2, 11, and 12) were seen, mainly at the lung side of the PTV. Consequently, the mean PTV dose appeared to be much lower than previously expected, on average $61.7 \mathrm{~Gy}(45.3-81.0 \mathrm{~Gy})$ as estimated by the CA appeared to be only $57.3 \mathrm{~Gy}$ $(44.2-77.6 \mathrm{~Gy})$ as estimated by the SA $(p<0.001)$. This difference was even larger if the EUD was used to estimate the tumour control probability, on average $61.3 \mathrm{~Gy}$ (44.9 $-80.4 \mathrm{~Gy}$ ) for the CA vs. $55.5 \mathrm{~Gy}(43.9-76.8 \mathrm{~Gy})$ for the SA ( $p<0.001)$, see Table 6.1.

With respect to the normal tissue parameters: the median MLD for plan 1-CA was 17.4 Gy (4.4 - 19.0 Gy) vs. $16.1 \mathrm{~Gy}(4.3-18.5 \mathrm{~Gy})$ for plan 1-SA (difference $-5.2 \pm 2.8 \%$ 
( $p<0.001)$, range -1.4 to $-0.1 \mathrm{~Gy}$ ). For the maximum spinal cord dose, these differences were very small, $54.0 \mathrm{~Gy}(17.9-54.0 \mathrm{~Gy})$ vs. $52.9 \mathrm{~Gy}(18.1-55.1 \mathrm{~Gy})$, respectively, a difference of $-0.5 \pm 1.5 \%$ ( $p=0.256$, range $-1.1-+1.3 \mathrm{~Gy}$ ), see Table 6.1.

\section{Comparison of plan 2-SA with plan 1-SA}

The second step was to estimate the effect on the dose distributions if new treatment plans were designed, using the SA, based on the same constraints. This analysis showed that the prescribed dose was lower with the newly designed treatment plans: 58.9 Gy $(43.7$ - $76.0 \mathrm{~Gy})$ and $54.9 \mathrm{~Gy}(43.9$ - $76.5 \mathrm{~Gy})$ for plan 1-SA and plan 2-SA $(p=0.211)$, respectively. However, when treatment plans were evaluated in terms of

Table 6.1: Patient characteristics and dose parameters.

\begin{tabular}{|c|c|c|c|c|c|c|c|c|c|c|c|c|c|c|c|c|}
\hline \multirow{3}{*}{ 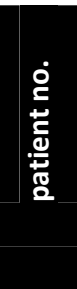 } & \multirow{3}{*}{ 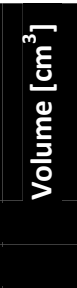 } & \multicolumn{8}{|c|}{$\begin{array}{l}\text { PTV } \\
\text { ose (PB) [Gy] and } \\
\text { orm dose (EUD) [Gy] }\end{array}$} & \multicolumn{2}{|c|}{$\begin{array}{c}\text { mean lung } \\
\text { dose [Gy] }\end{array}$} & \multicolumn{2}{|c|}{$\begin{array}{l}\text { maximum } \\
\text { spinal cord } \\
\text { dose [Gy] }\end{array}$} & \multicolumn{3}{|c|}{$\begin{array}{l}\text { dose-limiting } \\
\text { factor }\end{array}$} \\
\hline & & \multicolumn{2}{|c|}{ 1-CA } & \multicolumn{2}{|c|}{ 1-SA } & \multicolumn{2}{|c|}{ 2-SA } & \multicolumn{2}{|c|}{ 3-SA } & \multirow[t]{2}{*}{$1-C A$} & \multirow[t]{2}{*}{$1-S A$} & \multirow[t]{2}{*}{$1-\mathrm{CA}$} & \multirow[t]{2}{*}{ 1-SA } & \multirow[t]{2}{*}{$1-\mathrm{CA}$} & \multirow[t]{2}{*}{ 2-SA } & \multirow[t]{2}{*}{ 3-SA } \\
\hline & & PD & EUD & PD & EUD & PD & EUD & PD & EUD & & & & & & & \\
\hline 1 & 482 & 57.3 & 57.5 & 54.3 & 53.6 & 51.0 & 51.6 & 55.3 & & 19.0 & 17.7 & & 45.9 & & MLD & MLD \\
\hline 2 & 516 & 61.1 & 60.9 & 59.0 & 54.4 & 53.8 & 54.7 & 58.7 & 58.7 & 17.3 & 16.0 & 54.0 & 53.6 & SC & MLD & MLD \\
\hline 3 & 272 & 65.5 & 64.6 & 63.4 & 61.8 & 63.0 & 63.6 & 63.3 & 63.0 & 13.9 & 13.2 & 54.0 & 53.1 & SC & SC & SC \\
\hline 4 & 610 & 75.4 & 68.2 & 73.4 & 67.5 & 65.1 & 65.0 & 65.7 & 65.2 & 13.8 & 13.4 & 54.0 & 54.6 & SC & SC & SC \\
\hline 5 & 561 & 52.6 & 52.6 & 52.3 & 52.3 & 56.4 & 55.9 & 56.4 & 55.7 & & 4.3 & 54.0 & 54.3 & SC & SC & SC \\
\hline 6 & 487 & 77.2 & 78.3 & 76.0 & 76.8 & 76.5 & 77.1 & 76.5 & 76.1 & 19.0 & 18.5 & 17.9 & 18.1 & MLD & MPD & MPD \\
\hline 7 & 605 & 57.3 & 58.5 & 55.8 & 55.5 & 52.0 & 52.7 & 53.9 & 54.0 & 19.0 & 18.0 & 42.3 & 42.1 & MLD & MLD & MLD \\
\hline 8 & 411 & 53.6 & 53.5 & 53.0 & 52.2 & 49.3 & 49.7 & 56.3 & 55.9 & 18.7 & 18.1 & 54.0 & 53.2 & & MLD & MLD \\
\hline 9 & 435 & 65.5 & 65.6 & 62.8 & 61.1 & 62.7 & 63.8 & 66.1 & & 19.0 & 17.9 & 27.1 & 27.2 & & MLD & MLD \\
\hline 10 & 304 & 60.8 & 61.8 & 58.8 & 58.2 & 54.9 & 55.4 & 58.2 & 58.0 & 14.4 & 13.7 & 54.0 & 30.0 & SC & MLD & MLD \\
\hline 11 & 511 & 61.2 & 62.0 & 58.9 & 55.5 & 54.9 & 54.7 & 55.0 & 54.8 & 17.6 & 16.2 & 54.0 & 52.7 & SC & MLD & MLD \\
\hline 12 & 123 & 79.2 & 80.4 & 70.1 & 67.1 & 76.5 & 79.2 & 75.9 & & & 10.2 & 30.8 & 29.9 & & MPD & MPD \\
\hline 13 & 599 & 50.7 & 53.6 & 50.3 & 53.1 & 50.8 & 51.6 & 51.4 & 52.0 & 14.9 & 14.6 & 54.0 & 54.5 & SC & sc & SC \\
\hline 14 & 704 & 53.2 & 53.6 & 52.1 & 52.8 & 53.0 & 54.4 & 53.0 & 54.0 & 13.7 & 13.1 & 54.0 & 55.1 & & SC & SC \\
\hline 15 & 339 & 70.4 & 70.4 & 68.8 & 68.1 & 65.9 & 66.9 & 72.5 & 69.4 & 19.0 & 18.3 & 51.6 & 51.6 & MLD & MLD & MLD \\
\hline 16 & 859 & 44.6 & 44.9 & 43.7 & 43.9 & 43.7 & 43.7 & 44.6 & 45.3 & 19.0 & 18.3 & 45.0 & 44.9 & MLD & MLD & MLD \\
\hline $\begin{array}{l}\text { me- } \\
\text { dian }\end{array}$ & 499 & 61.0 & 61.3 & 58.9 & 58.4 & 54.9 & 58.7 & 57.3 & 59.9 & 17.4 & 16.1 & 54.0 & 52.9 & $\begin{array}{c}6 \\
\text { MLD }\end{array}$ & $\begin{array}{c}9 \\
\text { MLD }\end{array}$ & 9 \\
\hline range & $\begin{array}{l}123- \\
859\end{array}$ & $\begin{array}{c}44.6- \\
79.2\end{array}$ & $\begin{array}{l}44.9- \\
80.4\end{array}$ & 76.0 & 76.8 & $\begin{array}{l}43.7- \\
76.5\end{array}$ & 79.2 & 76.5 & 76.1 & 19.0 & $\begin{array}{l}4.3- \\
18.5\end{array}$ & $\begin{array}{l}17.9- \\
54.0\end{array}$ & 55.1 & $\begin{array}{c}9 \mathrm{SC} \\
1 \\
\mathrm{MPD}\end{array}$ & $\begin{array}{c}5 \mathrm{SC} \\
2 \\
\mathrm{MPD}\end{array}$ & $\begin{array}{c}5 \mathrm{SC} \\
2 \\
\mathrm{MPD}\end{array}$ \\
\hline
\end{tabular}

Abbreviations: $\mathrm{PTV}=$ planning target volume; $\mathrm{CA}=$ convolution algorithm; $\mathrm{SA}=$ superposition algorithm; $M L D=$ mean lung dose; $S C=$ spinal cord; $M P D=$ maximum prescribed dose. Bold numbers indicate the highest equivalent uniform dose values for the SA plans. 
the EUD of the PTV, these values appeared to be almost identical 55.5 Gy $(43.9-79.2$ Gy) vs. $55.1 \mathrm{~Gy}(43.7-79.2 \mathrm{~Gy})$ for plan 1-SA and plan 2-SA ( $p=0.796)$, respectively. Plan 1-SA was superior to plan 2-SA in 8 of the 16 patients, equal to plan 2-SA in 3 of the 16 patients (difference smaller than 1\%), and worse in 5 of the 16 patients (see Table 6.1).

For plan 2-SA, the restriction of the prescribed dose by the MLD was increased from 6 to 9 patients (Table 6.1), this is a result of the much larger radiation portals that had to be used to achieve full PTV coverage. Also a different beam set-up, i.e. another choice of beam angles, was necessary to achieve the lowest increase in dose in lung in the penumbra region around the PTV.

We found that the conventional beam set-up, consisting of three beams of which two were opposing, was not optimal and the set-up had to be changed to nonopposing beams. If 95\% PTV coverage was aimed at using the original gantry angles of plan 1, then field sizes had to be increased with 10 to $15 \mathrm{~mm}$ to yield adequate coverage. No difference between plan 1-SA and plan 2-SA was found that depended on the tumour location. Differences due to the size of the PTV were only observed for small peripheral tumours in the lung tissue without normal tissue constraints (e.g. patient 12). For these small volume tumours, plan 2-SA yielded a better coverage of the PTV and hence a higher PTV EUD due to the more accurate dose calculation algorithm that is necessary in highly inhomogeneous regions.
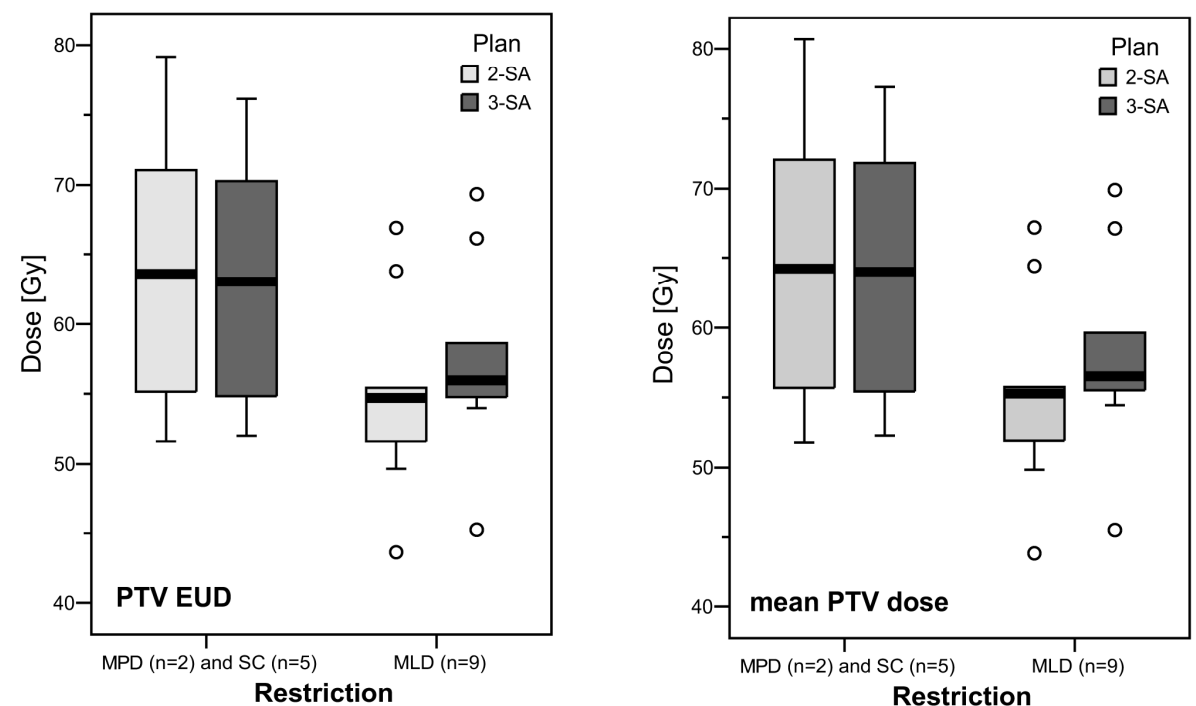

Figure 6.1: Box-plots of the equivalent uniform dose in the planning target volume (PTV EUD) (left) and mean PTV dose (right) between plans created by applying the ICRU constraints for dose homogeneity of 95\% isodose (plan 2-SA, black bars) and the plan with the relaxed homogeneity constraint at the lung side with a 90\% isodose coverage (plan 3-SA, gray bars). A significant increase in both EUD $(p=0.008)$ and mean dose $(p=0.008)$ was observed for the patient group with the mean lung dose (MLD) as the dose-limiting factor, while no gain is achieved for the group limited by the maximum prescribed dose (MPD) or the maximum dose to the spinal cord (SC), $p=0.128$ and $p=0.499$, respectively. 
In summary, plan 2-SA yielded a lower prescribed dose compared to plan 1-SA but due to a better dose homogeneity inside the PTV, the EUD remained approximately equal. However, in 8 out of the 16 patients the 2-SA plan yielded lower EUDs than the 1-SA plan, indicating that simple introduction of the SA without changing the constraints and dose criteria, may result in a worsening of treatment outcome in more than $50 \%$ of the patients. Therefore, we also analyzed the results when relaxing the $95 \%$ isodose criteria around the PTV at the lung side.

\section{Comparison of plan 3-SA with plan 2-SA}

In Figure 6.1 the differences between plan 2-SA and plan 3-SA are shown grouped according to the factor limiting dose-escalation (i.e. MLD vs. spinal cord or maximum prescribed dose). For the 9 patients with the MLD as the dose limiting factor in plan 2$\mathrm{SA}$, the maximum prescribed dose was increased by on average $3.6 \pm 2.4 \mathrm{~Gy}(p=0.008)$ by relaxing the PTV criterion at the lung side (plan 3-SA) to the $90 \%$ isodose surface. The mean PTV dose and the EUD increased on average by $2.9 \pm 1.9 \mathrm{~Gy}(p=0.008)$ and $2.7 \pm 1.8 \mathrm{~Gy}(p=0.008)$, respectively. Consequently, the underdosage of $5 \%$ in a small part of the PTV was compensated by a higher maximum prescribed dose. An example of the dose-volume histogram of the various plans for an individual patient (nr. 15) is shown in Figure 6.2.

For the 7 patients with the maximum prescribed dose or the spinal cord as the dose-limiting factor, no additional gain in prescribed dose $(+0.1 \pm 0.4 \mathrm{~Gy}, \mathrm{p}=0.249)$, mean PTV dose $(-0.6 \pm 1.5 \mathrm{~Gy}, \mathrm{p}=0.499)$ or in PTV EUD $(-0.8 \pm 1.4 \mathrm{~Gy}, \mathrm{p}=0.128)$ could be achieved. In fact, the small increase in prescribed dose led to a reduction in EUD, due to underdosage in parts of the PTV.

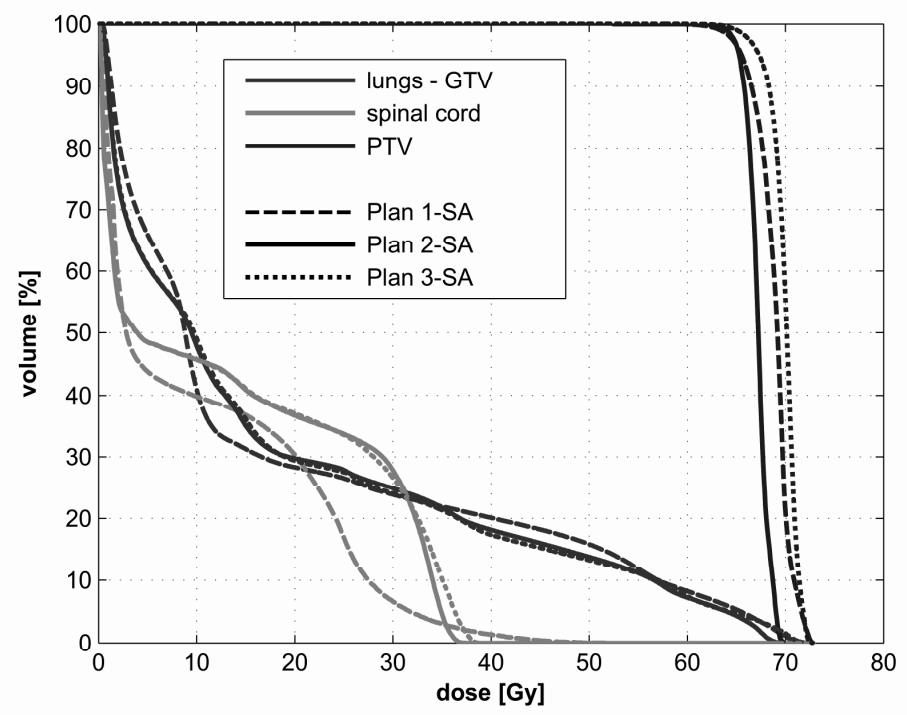

Figure 6.2: Example of a typical dose-volume histogram (DVH) (patient 15) with the mean lung dose (MLD) as dose limiting factor for dose-escalation. An additional gain of $2.5 \mathrm{~Gy}$ in terms of EUD is achieved between plan 2-SA and plan 3-SA. 


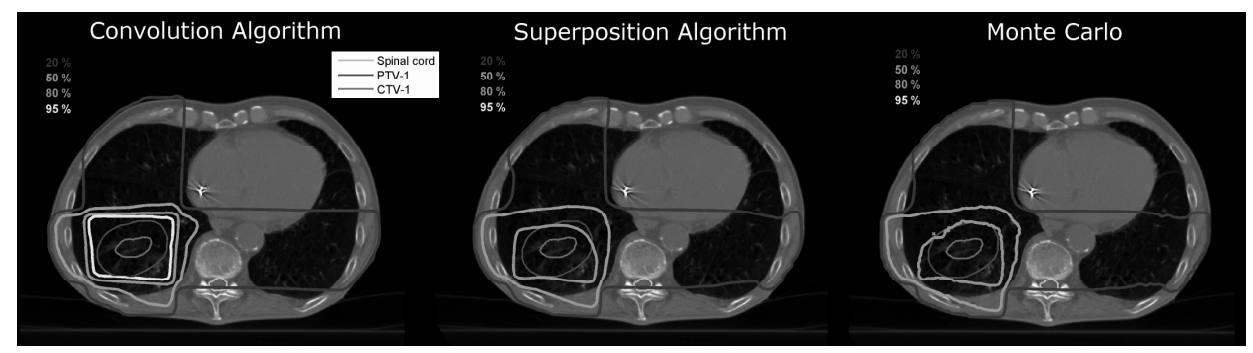

Figure 6.3: Example of the dose distributions of patient 12 using the various dose calculation algorithms. For each calculation the same beam set-up is applied with identical number of monitor units. Note that the MC or SA algorithm do not even reach a coverage of the $80 \%$ isodose around the PTV; the $100 \%$ isodose corresponds to a dose level of $79.2 \mathrm{~Gy}$.

The mean PTV dose showed good correlation $\left(R^{2}=0.998\right)$ with the calculated PTV EUD for a dose range between $90 \%$ and $107 \%$. If a coverage of the PTV with the $95 \%$ (or $90 \%$ ) and $107 \%$ isodose surface is achieved, the PTV EUD is only $1.0 \%$ (or $1.3 \%$ ) lower compared to the mean PTV dose.

\section{Ranking treatment plan 1-SA, 2-SA and 3-SA}

Ranking the three SA treatment plans showed that plan 1-SA yielded the highest PTV EUD in five patients, plan 2-SA in five patients and plan 3-SA in six patients. Thus, there is not one single plan that yields the best results for all patients.

\section{Dose verification}

An example of the dose distribution of plan 1 (patient 12) using the CA, SA and MC dose calculation is shown in Figure 6.3. For the 5 patients analyzed with all 3 dose calculation algorithms, PTV EUD, MLD and maximum dose in the spinal cord are shown in Table 6.2.

The PTV EUD using the CA is overestimated compared to the EUD calculated using a more advanced algorithm. If the more accurate algorithms are used, the EUD decreases by $-5.6 \pm 8.0 \%$ for the SA and $-5.1 \pm 7.1 \%$ for the MC method, compared to the CA. The average difference between EUD of the SA and MC was small: $-0.3 \pm 1.1 \%$.

The average MLD of the five CA plans was $14.9 \mathrm{~Gy}(11.5-19.0 \mathrm{~Gy})$ and reduced by $5.1 \pm 4.2 \%$ to $14.6 \mathrm{~Gy}(10.2-18.3 \mathrm{~Gy})$ for the SA. The MLD calculated using the MC method was $15.4 \mathrm{~Gy}(10.8-19.0 \mathrm{~Gy})$ and did not largely differ $(0.4 \pm 3.4 \%)$ compared to the value obtained with the CA method. The difference between SA and MC was somewhat larger: $4.4 \pm 1.0 \%$. The maximum dose, expressed as $D_{0.1 \%}$, in the spinal cord did not vary between the 3 dose distributions: $47.9 \mathrm{~Gy}(30.5-53.6 \mathrm{~Gy}), 48.1 \mathrm{~Gy}$ (29.6 $54.0 \mathrm{~Gy})$ and $46.1 \mathrm{~Gy}(32.1$ - $55.6 \mathrm{~Gy})$ for the CA, SA and MC dose distribution, respectively. 
Table 6.2 Difference between CA, SA, and MC dose calculation for the 5 patients analyzed.

\begin{tabular}{|c|c|c|c|c|c|c|c|c|c|}
\hline \multirow[t]{2}{*}{$\begin{array}{l}\text { patient } \\
\text { no. }\end{array}$} & \multicolumn{3}{|c|}{\begin{tabular}{|c} 
PTV \\
equivalent uniform dose \\
[Gy]
\end{tabular}} & \multicolumn{3}{|c|}{$\begin{array}{c}\text { mean lung dose } \\
\text { [Gy] }\end{array}$} & \multicolumn{3}{|c|}{$\begin{array}{c}\text { maximum dose }\left(D_{0.1 \%}\right) \\
\text { in the spinal cord } \\
{[G y]}\end{array}$} \\
\hline & 1-CA & 1-SA & 1-MC & $1-\mathrm{CA}$ & 1-SA & 1-MC & $1-\mathrm{CA}$ & 1-SA & 1-MC \\
\hline 12 & 80.4 & 67.1 & 66.0 & 11.5 & 10.2 & 10.8 & 30.5 & 29.6 & 32.1 \\
\hline 13 & 53.6 & 53.1 & 52.3 & 14.9 & 14.6 & 15.4 & 53.6 & 54.0 & 55.6 \\
\hline 14 & 53.6 & 52.8 & 53.0 & 13.7 & 13.1 & 13.8 & 51.2 & 52.1 & 51.8 \\
\hline 15 & 70.4 & 68.1 & 68.5 & 19.0 & 18.3 & 19.0 & 47.9 & 48.1 & 43.2 \\
\hline 16 & 44.9 & 43.9 & 44.0 & 19.0 & 18.3 & 18.9 & 45.0 & 44.9 & 46.1 \\
\hline median & 53.6 & 53.1 & 53.0 & 14.9 & 14.6 & 15.4 & 47.9 & 48.1 & 46.1 \\
\hline range & $\begin{array}{c}44.9- \\
80.4\end{array}$ & $\begin{array}{l}43.9- \\
68.1\end{array}$ & $\begin{array}{c}44.0- \\
68.5\end{array}$ & $\begin{array}{c}11.5- \\
19.0\end{array}$ & $\begin{array}{c}10.2- \\
18.3\end{array}$ & $\begin{array}{c}10.8- \\
19.0\end{array}$ & $\begin{array}{c}30.5- \\
53.6\end{array}$ & $\begin{array}{c}29.6- \\
54.0\end{array}$ & $\begin{array}{c}32.1- \\
55.6\end{array}$ \\
\hline
\end{tabular}

Abbreviations: PTV = planning target volume; $\mathrm{CA}=$ convolution algorithm; $\mathrm{SA}=$ superposition algorithm; $\mathrm{MC}=$ Monte Carlo; $\mathrm{D}_{0.1 \%}=$ maximum dose to $0.1 \%$ of the volume

\section{DISCUSSION}

\section{ICRU criteria and dose calculation algorithm}

Ranking the different treatment plans showed large individual variation; we found it not possible to predict in advance which algorithm or treatment plan will result in the highest tumour control probability. If the ICRU dose homogeneity restrictions (i.e. dose inside the PTV must be within the range of $95 \%$ - $107 \%$ of the prescribed dose) are followed, it is clear that the CA algorithm calculates a dose in the PTV that is within the restrictions. However, in reality a much more heterogeneous dose distribution is delivered that in most cases does not comply with the ICRU criteria. The recalculation of these dose distributions using a more advanced algorithm (e.g. SA or MC) results in a more realistic dose distribution that reveals coverage of the PTV for some patients of only the $60 \%$ or $70 \%$ isodose surface. This also shows that calculating and prescribing dose using a simple algorithm is not suitable for lung cancer treatments. For accurate estimation of the dose calculation in regions with high density gradients such as the thorax, more advanced algorithms are necessary. ${ }^{1-7}$

While plans designed using these more advanced algorithms have a better dose homogeneity inside the target volume, these plans usually result in a lower prescribed dose compared the prescribed dose achieved with the simple algorithms, due to higher MLDs. Therefore a slight relaxation of the PTV coverage for the plans designed using the more advanced algorithms was introduced that resulted in a maximum prescribed dose similar to the dose calculated using the simple algorithms. In reality these plans have a better coverage of the PTV, than the plans designed using the apparent $95 \%$ coverage by assuming an evaluation of the wrong calculated dose distribution using the simple algorithm.

The superposition algorithm used in this study has shown to be in good agreement with the verification calculations performed using Monte Carlo simulations for both 
the PTV and the normal tissue. This was also shown by Morgan et al. ${ }^{6}$ who verified the same superposition algorithm against another type of Monte Carlo calculations using a phantom study. The reduction of the prescribed dose of on average $3.4 \%$ is also in agreement with Knöös et al. ${ }^{8}$, who found a decrease around $2.5-3.7 \%$ for a lung case. Irvine et al $^{28}$ showed the same amount in dose reduction if plans were recalculated using a more advanced algorithm for various thoracic cases. They also reported a lower minimum dose inside the PTV up to $23 \%$ for the recalculated plans. These findings are in agreement with the results found in this study.

Some discrepancies have been observed for the MLD of plan 1-MC, which might be due to the fact that the Monte Carlo based dose reconstruction procedure only has a single photon energy spectrum that is the same for the open and blocked fields. Hence, some small differences in the dose calculation behind the blocked fraction of the field can be expected. ${ }^{27}$ The MLD calculated using the MC method will thus in reality be somewhat lower and probably comparable to the MLD calculated using the SA algorithm.

A previous study performed by De Jaeger et al. ${ }^{4}$ showed a larger difference between two different dose calculation algorithms (i.e. an algorithm applying an Equivalent Path Length (EPL) correction and the Convolution-Superposition (CS) algorithm). These authors showed a decrease in MLD up to $16 \%$ by switching from the simple to the more advanced algorithm. This difference is larger than the difference of approximately $5 \%$ found in this study. There may be several reasons for this observation. First, the algorithm with the EPL correction is an even simpler algorithm than the CA algorithm used in this study. Second, because the EPL and the CS algorithm were implemented in a different TPS in the study of De Jaeger et al. ${ }^{4}$, the beam modelling data that are used as the starting point in their study is likely to cause some additional differences, whereas in our study the CA and the SA algorithm are both implemented in the same TPS using the same beam modelling data. Therefore the only possible difference in our study is the dose calculation engine and not the beam modelling data.

\section{EUD, TCP and mean PTV dose}

Various metrics have been presented in the literature to describe an inhomogeneous dose distribution inside a target volume or an organ at risk. The most simple parameters are the minimum, mean and maximum dose inside the PTV. ICRU50 poses criteria on these parameters and recommended that dose heterogeneity must not exceed $95 \%$ and $107 \%$ of the prescribed dose inside the PTV. To assess the impact of more heterogeneous dose distributions, concepts such as equivalent uniform dose (EUD) or tumour control probability (TCP) are often used. The EUD is a measure of the dose delivered to a volume that would result in the same cell kill as a homogeneous dose. In this work, the EUD has been applied under assumption that there exists a homogeneous distribution of tumour cells. TCP is generally calculated from the EUD by introducing some additional tumour specific or radiobiological parameters like alpha/beta ratios or TD50. The exact values of these parameters are under debate and usually have a large variation in the actual value. ${ }^{29}$ Therefore such metrics might serve as a more clinical related outcome parameter than mean dose, but should be used with caution. As long 
as the dose inhomogeneity is relatively small, even for a variation between $90-107 \%$ instead of $95 \%-107 \%$, the EUD is very similar to the mean PTV dose, but larger differences may occur when the dose inhomogeneity increases. ${ }^{24}$

Another important issue that must be taken into account are the margin from CTV to PTV. This margin is used to incorporate possible tumour motion and daily set-up variations into the treatment planning process for fractionated treatment. This margin is designed in such a way that if the PTV is covered in the planning situation (single planning CT scan), then the dose delivered to the CTV during the entire course of treatment is represented by this dose value. One should however be careful in calculating and interpretating EUD and TCP values, since both the models and the input parameters have uncertainties. For example the homogeneous clonogenic cell density that is often assumed for the calculation might be the actual situation for the GTV, but the tumour cell density in the CTV or PTV might be different. We have chosen to calculate the EUD inside the PTV to get an estimate of the coverage and impact of heterogeneities inside the treated volume. It is not a direct estimation of the dose to the tumour because of the margins used, but at the moment the PTV is the recommended volume to incorporate possible movement of the tumour, daily set-up of the patient and all other uncertainties occurring during treatment.

\section{Relaxation of ICRU PTV coverage criterion}

The application of the ICRU criterion for dose homogeneity inside the PTV restricts the prescribed dose or EUD to the PTV. For dose-escalation, a relaxation of the lower bound coverage criterion to $90 \%$ at the lung side is, in cases where the MLD is the dose-limiting factor, a possibility to escalate to higher dose levels. Schwarz et al. ${ }^{30}$ and Engelsman et al. ${ }^{24}$ already showed that the removal of the maximum or minimum dose constraint inside the PTV, respectively, could yield a higher EUD compared to the plans designed following the ICRU guidelines. In IMRT optimization procedures this constraint can be removed from the plan objectives. We used forward planned conformal radiotherapy and applied a relaxation of the isodose coverage at the lung side to create this inhomogeneous dose distribution, which requires a slight change and some additional user interaction during treatment planning. However, this is possible without adapting the rest of the treatment planning procedure. We decided to accept only a minor dose-inhomogeneity, because it is likely that the uncertainties in the EUD model are currently too large to take large dose inhomogeneities into account. In addition, in this range the EUD is similar to the mean PTV dose, which is a much more simple parameter that can easily be calculated by most planning systems.

The amount of increase in tumour control probability (TCP) by only slightly relaxing the ICRU criteria from $95 \%$ to $90 \%$ coverage at the lung side of the PTV can be estimated. For example for the patient group with MLD restriction, the average PTV EUD is increased from $54.8 \mathrm{~Gy}$ to $57.5 \mathrm{~Gy}$ which relates to an increase in TCP of $12.4 \%$ to $17.2 \%$, for $a D_{50}=70 \mathrm{~Gy}$ and $a \gamma=2.0$ by using TCP $=\left(1+\left(D_{50} / D\right)^{4 \gamma}\right)^{-1} \cdot{ }^{31}$ In individual patients (i.e. Patient 8) a gain as large as $6.3 \mathrm{~Gy}$ more than doubles the expected TCP from $6.0 \%$ to $14.2 \%$. 


\section{Clinical implementation of the SA}

Since the SA calculates a more accurate dose distribution than the $\mathrm{CA}$, it is obviously very attractive to apply this advanced algorithm in clinical practice. However, we showed that not a single plan yields the highest PTV EUD for all patients. Ideally, three plans should therefore be made for each patient (1-SA, 2-SA and 3-SA), and the plan with the highest PTV EUD should be selected. However, since this is extremely impractical in clinical practice, we analyzed the consequences of choosing two options. Option 1 is to only use plan 2-SA, and option 2 is to use plan 2-SA with changing into 3SA when the MLD is the dose-limiting factor, instead of the 1-SA plan.

If we would choose for option 1, i.e. plan 2-SA, the average PTV EUD would not differ from the PTV EUD of plan 1-SA, but in 9 out of 16 patients the PTV EUD would be on average $1.8 \pm 0.9$ Gy lower (range $-2.8--0.3 \mathrm{~Gy}$ ) than with plan 1-SA. In the other 7 patients, the PTV EUD would be on average $3.7 \pm 4.3 \mathrm{~Gy}$ higher (range $0.3-12.1 \mathrm{~Gy}$ ) than with plan 1-SA.

If option 2 is chosen, i.e. plans 2-SA with changing into plan 3-SA when the MLD constraint is met, the PTV EUD would be on average $1.9 \pm 3.5$ Gy (range $-2.6-+12.1$ Gy) higher compared to plan 1-SA (range $-2.3-+8.3 \mathrm{~Gy}$ ). Nevertheless, in 5 patients, a higher PTV EUD could be obtained with plan 1-SA, but this difference was very small (on average $-1.2 \pm 0.9 \mathrm{~Gy}$, range $-2.6--0.2 \mathrm{~Gy}$ ). In the other 11 patients, an important increase in PTV EUD of on average $3.3 \pm 3.3 \mathrm{~Gy}$, with a range from 0.3 to $12.1 \mathrm{~Gy}$, could be obtained.

These data show that by simply introducing the SA without adapting the PTVcoverage criterion, 9 out of the 16 of the patients would yield a lower PTV EUD than using the CA (i.e. plan 1-SA). By relaxing the $95 \%$ coverage criterion, a considerable gain could be obtained in PTV EUD. Although still 5 out of the 16 patients do not reach the PTV EUD of the 1-SA plan, the reduction in PTV EUD is, however, quite small: at maximum $2.6 \mathrm{~Gy}$. On the other hand, in the 11 patients who do reach a higher PTV EUD with the 2-SA or 3-SA plan, impressive gains could be obtained up till $12.1 \mathrm{~Gy}$. Therefore, it is our opinion that the balance between positive and negative effects is clearly in favour of the 2-SA plan, and changing into 3-SA in case of MLD restriction.

\section{CONCLUSIONS AND RECOMMENDATIONS FOR IMPLEMENTATION IN CLINICAL ROUTINE}

Some recommendations can be made concerning the implementation of a more advanced algorithm in clinical routine. First, the use of such an algorithm leads to more accurate dose distributions in regions having tissue inhomogeneities. For prospective as well as retrospective evaluation of (recalculated) dose distributions this is a valuable step forward.

Second, to obtain the optimal plan using the more advanced algorithm usually larger field sizes are required to achieve adequate PTV coverage. Also a different beam set-up, i.e. another choice of beam angles, is generally necessary to obtain the lowest MLD and smallest increase of the penumbra width around the PTV in the lung tissue. 
A third point concerns the dose delivered in the target volume, either expressed as a mean PTV dose or PTV EUD. As long as the dose heterogeneity inside the PTV is not large, i.e. the $90 \%$ isodose covering the PTV, the mean dose can be used as an accurate surrogate for the EUD. For larger dose inhomogeneities in the PTV this relation is no longer valid and a larger difference between EUD and mean dose will occur. ${ }^{24}$

Finally, if the mean lung dose is the normal tissue constraint that restricts the escalation of the tumour dose to a higher amount, then the ICRU guidelines should be relaxed in such a way that coverage of the PTV at the lung side is only $90 \%$. This will lead to an additional gain in mean target dose or EUD, because a field size reduction at this side will have a larger impact on the MLD than on the mean dose or EUD of the PTV. However, if the normal tissue constraint is the maximum dose in the spinal cord, a relaxation of the dose coverage around the PTV has no effect because field size reduction will in most cases not alter the maximum dose in the spinal cord.

\section{REFERENCES}

${ }^{1}$ N. Papanikolaou, J. Battista, A. Boyer, C. Kappas, E. Klein, T.R. Mackie, M. Sharpe, and J. Van Dyk, AAPM Report No 85, Tissue Inhomogeneity Corrections for Megavoltage Photon Beams. AAPM Report No 85, Task Group No 65 of the Radiation Therapy Committee of the American Association of Physicists in Medicine. 2004, Madison, WI: Medical Physics Publishing.

${ }^{2}$ U. Haedinger, T. Krieger, M. Flentje, and J. Wulf, "Influence of calculation model on dose distribution in stereotactic radiotherapy for pulmonary targets," Int J Radiat Oncol Biol Phys 61, 239-249 (2005).

${ }^{3}$ M. Engelsman, E.M. Damen, P.W. Koken, A.A. van 't Veld, K.M. van Ingen, and B.J. Mijnheer, "Impact of simple tissue inhomogeneity correction algorithms on conformal radiotherapy of lung tumours," Radiother Oncol 60, 299-309 (2001).

${ }^{4}$ K. De Jaeger, M.S. Hoogeman, M. Engelsman, Y. Seppenwoolde, E.M. Damen, B.J. Mijnheer, L.J. Boersma, and J.V. Lebesque, "Incorporating an improved dose-calculation algorithm in conformal radiotherapy of lung cancer: re-evaluation of dose in normal lung tissue," Radiother Oncol 69, 1-10 (2003).

${ }^{5}$ C.M. Bragg, K. Wingate, and J. Conway, "Clinical implications of the anisotropic analytical algorithm for IMRT treatment planning and verification," Radiother Oncol 86, 276-284 (2008).

${ }^{6}$ A.M. Morgan, T. Knoos, S.G. McNee, C.J. Evans, and D.I. Thwaites, "Clinical implications of the implementation of advanced treatment planning algorithms for thoracic treatments," Radiother Oncol 86, 48-54 (2008).

${ }^{7}$ A. Fogliata, E. Vanetti, D. Albers, C. Brink, A. Clivio, T. Knoos, G. Nicolini, and L. Cozzi, "On the dosimetric behaviour of photon dose calculation algorithms in the presence of simple geometric heterogeneities: comparison with Monte Carlo calculations," Phys Med Biol 52, 1363-1385 (2007).

${ }^{8}$ T. Knoos, E. Wieslander, L. Cozzi, C. Brink, A. Fogliata, D. Albers, H. Nystrom, and S. Lassen, "Comparison of dose calculation algorithms for treatment planning in external photon beam therapy for clinical situations," Phys Med Biol 51, 5785-5807 (2006).

${ }^{9}$ F. Garcia-Vicente, A. Minambres, I. Jerez, I. Modolell, L. Perez, and J.J. Torres, "Experimental validation tests of fast Fourier transform convolution and multigrid superposition algorithms for dose calculation in low-density media," Radiother Oncol 67, 239-249 (2003). 
${ }^{10}$ W.J. van Elmpt, S.M. Nijsten, A.L. Dekker, B.J. Mijnheer, and P. Lambin, "Treatment verification in the presence of inhomogeneities using EPID-based three-dimensional dose reconstruction," Med Phys 34, 2816-2826 (2007).

${ }^{11} \mathrm{C}$. Scholz, C. Schulze, U. Oelfke, and T. Bortfeld, "Development and clinical application of a fast superposition algorithm in radiation therapy," Radiother Oncol 69, 79-90 (2003).

${ }^{12}$ ICRU, International Commission on Radiation Units and Measurements Report 50: Prescribing, recording, and reporting photon beam therapy. 1993: Bethesda.

${ }^{13}$ J.S. Belderbos, W.D. Heemsbergen, K. De Jaeger, P. Baas, and J.V. Lebesque, "Final results of a Phase I/II dose escalation trial in non-small-cell lung cancer using three-dimensional conformal radiotherapy," Int J Radiat Oncol Biol Phys 66, 126-134 (2006).

${ }^{14}$ J. Bradley, M.V. Graham, K. Winter, J.A. Purdy, R. Komaki, W.H. Roa, J.K. Ryu, W. Bosch, and B. Emami, "Toxicity and outcome results of RTOG 9311: a phase I-II dose-escalation study using three-dimensional conformal radiotherapy in patients with inoperable non-small-cell lung carcinoma," Int J Radiat Oncol Biol Phys 61, 318-328 (2005).

${ }^{15}$ J.A. Hayman, M.K. Martel, R.K. Ten Haken, D.P. Normolle, R.F. Todd, 3rd, J.F. Littles, M.A. Sullivan, P.W. Possert, A.T. Turrisi, and A.S. Lichter, "Dose escalation in non-small-cell lung cancer using three-dimensional conformal radiation therapy: update of a phase I trial," J Clin Oncol 19, 127-136 (2001).

${ }^{16}$ F.M. Kong, R.K. Ten Haken, M.J. Schipper, M.A. Sullivan, M. Chen, C. Lopez, G.P. Kalemkerian, and J.A. Hayman, "High-dose radiation improved local tumor control and overall survival in patients with inoperable/unresectable non-small-cell lung cancer: long-term results of a radiation dose escalation study," Int J Radiat Oncol Biol Phys 63, 324-333 (2005).

${ }^{17}$ M. Mehta, R. Scrimger, R. Mackie, B. Paliwal, R. Chappell, and J. Fowler, "A new approach to dose escalation in non-small-cell lung cancer," Int J Radiat Oncol Biol Phys 49, 23-33 (2001).

${ }^{18}$ F.M. Kong, J.A. Hayman, K.A. Griffith, G.P. Kalemkerian, D. Arenberg, S. Lyons, A. Turrisi, A. Lichter, B. Fraass, A. Eisbruch, T.S. Lawrence, and R.K. Ten Haken, "Final toxicity results of a radiation-dose escalation study in patients with non-small-cell lung cancer (NSCLC): predictors for radiation pneumonitis and fibrosis," Int J Radiat Oncol Biol Phys 65, 1075-1086 (2006).

${ }^{19}$ E.D. Yorke, A. Jackson, K.E. Rosenzweig, L. Braban, S.A. Leibel, and C.C. Ling, "Correlation of dosimetric factors and radiation pneumonitis for non-small-cell lung cancer patients in a recently completed dose escalation study," Int J Radiat Oncol Biol Phys 63, 672-682 (2005).

${ }^{20} \mathrm{M}$. Miften, M. Wiesmeyer, S. Monthofer, and K. Krippner, "Implementation of FFT convolution and multigrid superposition models in the FOCUS RTP system," Phys Med Biol 45, 817-833 (2000).

${ }^{21}$ S.L. Kwa, J.V. Lebesque, J.C. Theuws, L.B. Marks, M.T. Munley, G. Bentel, D. Oetzel, U. Spahn, M.V. Graham, R.E. Drzymala, J.A. Purdy, A.S. Lichter, M.K. Martel, and R.K. Ten Haken, "Radiation pneumonitis as a function of mean lung dose: an analysis of pooled data of $540 \mathrm{pa}-$ tients," Int J Radiat Oncol Biol Phys 42, 1-9 (1998).

${ }^{22}$ A. van Baardwijk, G. Bosmans, L. Boersma, S. Wanders, A. Dekker, A.M. Dingemans, G. Bootsma, W. Geraedts, C. Pitz, J. Simons, P. Lambin, and D. De Ruysscher, "Individualized Radical Radiotherapy of Non-Small-Cell Lung Cancer Based on Normal Tissue Dose Constraints: A Feasibility Study," Int J Radiat Oncol Biol Phys in press, (2008).

${ }^{23}$ L.B. Marks, J. Garst, M.A. Socinski, G. Sibley, A.W. Blackstock, J.E. Herndon, S. Zhou, T. Shafman, A. Tisch, R. Clough, X. Yu, A. Turrisi, M. Anscher, J. Crawford, and J. Rosenman, "Carboplatin/paclitaxel or carboplatin/vinorelbine followed by accelerated hyperfractionated conformal radiation therapy: report of a prospective phase I dose escalation trial from the Carolina Conformal Therapy Consortium," J Clin Oncol 22, 4329-4340 (2004). 
${ }^{24}$ M. Engelsman, P. Remeijer, M. van Herk, J.V. Lebesque, B.J. Mijnheer, and E.M. Damen, "Field size reduction enables iso-NTCP escalation of tumor control probability for irradiation of lung tumors," Int J Radiat Oncol Biol Phys 51, 1290-1298 (2001).

${ }^{25} \mathrm{~A}$. Niemierko, "Reporting and analyzing dose distributions: a concept of equivalent uniform dose," Med Phys 24, 103-110 (1997).

${ }^{26}$ W.J. van Elmpt, S.M. Nijsten, R.F. Schiffeleers, A.L. Dekker, B.J. Mijnheer, P. Lambin, and A.W. Minken, "A Monte Carlo based three-dimensional dose reconstruction method derived from portal dose images," Med Phys 33, 2426-2434 (2006).

${ }^{27}$ W. van Elmpt, S. Nijsten, B. Mijnheer, A. Dekker, and P. Lambin, "The next step in patientspecific QA: 3D dose verification of conformal and intensity-modulated RT based on EPID dosimetry and Monte Carlo dose calculations," Radiother Oncol 86, 86-92 (2008).

${ }^{28} \mathrm{C}$. Irvine, A. Morgan, A. Crellin, A. Nisbet, and I. Beange, "The clinical implications of the collapsed cone planning algorithm," Clin Oncol (R Coll Radiol) 16, 148-154 (2004).

${ }^{29}$ S. Webb and A.E. Nahum, "A model for calculating tumour control probability in radiotherapy including the effects of inhomogeneous distributions of dose and clonogenic cell density," Phys Med Biol 38, 653-666 (1993).

${ }^{30}$ M. Schwarz, M. Alber, J.V. Lebesque, B.J. Mijnheer, and E.M. Damen, "Dose heterogeneity in the target volume and intensity-modulated radiotherapy to escalate the dose in the treatment of non-small-cell lung cancer," Int J Radiat Oncol Biol Phys 62, 561-570 (2005).

${ }^{31}$ J.F. Fowler, W.A. Tome, J.D. Fenwick, and M.P. Mehta, "A challenge to traditional radiation oncology," Int J Radiat Oncol Biol Phys 60, 1241-1256 (2004). 
TREATMENT VERIFICATION AND IN VIVO DOSIMETRY 



\section{CHAPTER}

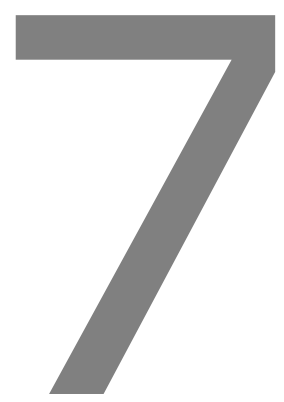

Calibration of megavoltage cone-beam CT for radiotherapy dose calculations: Correction of cupping artifacts and conversion of CT-numbers to electron density

Steven Petit, Wouter van Elmpt, Sebastiaan Nijsten, Philippe Lambin and André Dekker

Published in: Medical Physics 35(5):849-865, 2008. 


\begin{abstract}
Megavoltage cone-beam CT (MV CBCT) is used for 3D imaging of the patient anatomy on the treatment table prior to or just after radiotherapy treatment. To use MV CBCT images for radiotherapy dose calculation purposes, reliable electron density (ED) distributions are needed. Patient scatter, beam hardening and softening effects result in cupping artifacts in MV CBCT images and distort the CT number to ED conversion.

A method based on transmission images is presented to correct for these effects without using prior knowledge of the object's geometry. The scatter distribution originating from the patient is calculated with pencil beam scatter kernels that are fitted based on transmission measurements. The radiological thickness is extracted from the scatter subtracted transmission images and is then converted to the primary transmission used in the cone-beam reconstruction. These corrections are performed in an iterative manner, without using prior knowledge regarding the geometry and composition of the object. The method was tested using various homogeneous and inhomogeneous phantoms with varying shapes and compositions, including a phantom with different electron density inserts, phantoms with large density variations and an anthropomorphic head phantom.

For all phantoms, the cupping artifact was substantially removed from the images and a linear relation between CT number and electron density was found. Accurate conversion to ED could be made with difference that were reduced from up to $0.30 \mathrm{ED}$ units for the uncorrected images compared to differences in the corrected images smaller than 0.03 ED units for the majority of analyzed electron densities; the residual difference is equal to the amount of noise in the images. The ED distributions were evaluated in terms of absolute dose calculation accuracy for homogeneous cylinders of different size; errors decreased from $7 \%$ to below $1 \%$ in the center of the objects for the uncorrected and corrected images, respectively, and maximum differences were reduced from $17 \%$ to $2 \%$, respectively.

The presented method corrects the MV CBCT images for cupping artifacts and extracts reliable ED information of objects with varying geometries and composition, making these corrected MV CBCT images suitable for accurate dose calculation purposes.
\end{abstract}




\section{INTRODUCTION}

With the introduction of three-dimensional (3D) conformal radiotherapy and intensitymodulated radiotherapy (IMRT), irradiation strategies have become more and more complicated and dose gradients have become steeper. Accurate positioning of the patient and verification of the treatment delivery have thus become more important, since a small misalignment of the patient set up can have large consequences on the effectiveness of the treatment. On-line imaging prior to treatment is currently used for geometric verification of the patient position, but these images can also be used for a verification of the dose delivered in the actual patient anatomy at the time of treatment.

To perform this dosimetric verification, several methods are proposed in the literature to reconstruct the 3D patient dose distribution based on portal images and an electron density (ED) map of the patient. ${ }^{1-8}$ The planning CT scan should preferably not be used to provide the ED map, because differences in patient position and anatomy other than rigid body transformations can occur between the time of the planning CT scan and treatment fractions, due to for example, organ motion and weight loss. Online anatomical information of the patient on the treatment table is required. A first method was accomplished using helical tomotherapy with on-board imaging capabilities. Kapatoes et al. ${ }^{3}$ reconstructed the 3D dose distribution with tomotherapy treatment based on transmission measurements and a CT image of the patient in treatment position. A second option is to use a CT scanner in the treatment suite, that shares the same couch as the treatment unit. ${ }^{9}$ The third method to obtain on-line anatomical information of the patient on the treatment table ${ }^{10,11}$, is to combine a large number of portal images, measured from different angles around the patient, to yield a 3D image. This technique is referred to as cone-beam CT (CBCT). СВCT can be divided into two categories depending on the energy of the photons, KV CBCT and MV CBCT. To use CBCT for dose calculation purposes, the scanner must, preferably, be attached to the treatment unit to avoid differences in patient position between the CBCT acquisition and treatment. Whereas for KV CBCT an additional kV source and detector is needed, MV CBCT can be performed with the linear accelerator (linac) in combination with an Electronic Portal Imaging Device (EPID) sharing the same isocenter at the linac as the actual treatment. A disadvantage is, that the contrast in the MV CBCT images is lower compared to that in the KV CBCT images. ${ }^{5,12,13}$

In order to use MV CBCT images for 3D dose calculations, the images must be calibrated to electron density (ED). This is not straightforward. The Feldkamp cone-beam algorithm, ${ }^{14}$ used to reconstruct the 2D portal images to a 3D image, assumes a linear relation between log attenuation and object electron density with thickness, which is valid in case of a mono-energetic MV photon beam and no scatter radiation. However, in $\mathrm{MV}$ CBCT this linear relation is disturbed by three factors. First, the scatter radiation originating from the object contributes to the signal in the portal images. Second, the energetic spectrum of the treatment beam results in beam hardening as the beam travels through the object and a shift in beam spectrum towards lower energies for off-axis locations, referred to as beam softening. Third, the response of the EPID to radiation depends on the energy of the photons incident on the detector. If the portal 
images are not corrected before $\mathrm{CBCT}$ reconstruction the $\mathrm{MV}$ CBCT images will experience cupping artifacts that can lead to errors in dose calculation. ${ }^{1,15}$

A number of different methods have been proposed to reduce the cupping artifacts in MV CBCT images in order to obtain reliable electron densities. Chen et al. ${ }^{1}$ derived a spatially dependent cupping correction function with the MV CBCT of a large water cylinder. However, details about the exact method they used are not provided. In a more detailed paper by Morin et al. ${ }^{16}$ of the same group, the cupping artifact of a $16 \mathrm{~cm}$ diameter water cylinder was characterized using multiple ellipsoid shapes of different size centered around the same point in space. The ellipsoid shapes are associated with different correction factors. The CT numbers of the voxels that are positioned on the surface of one ellipsoid are multiplied with the same correction factor to account for the cupping artifact. The remaining cupping artifact of the MV CBCT of a head-and-neck patient was smaller than $5 \%$. In order to correct cupping artifacts of MV CBCTs of different body parts centered on different off-axis locations, multiple phantoms need to be scanned and different sets of ellipsoid shapes need to be acquired.

Spies et al. ${ }^{17}$ used Monte Carlo based scatter kernels to remove the scatter from the MV CB portal images and applied a linear quadratic model accounting for pixel-topixel variations in sensitivity and beam hardening. A CCD camera-based EPID was used with a total dose of $636 \mathrm{cGy}$. The overall cupping artifact reduced from $30 \%$ to $8 \%$, although discrepancies for cortical bone were still large, up to $17 \%$.

Dose calculations using kV CB CT scans have also been described. ${ }^{18-20}$ Yoo et al. ${ }^{19}$ recommended mounting a bowtie filter to improve image quality, yielding dosimetric results equal to those based on CT plans. Yang et al. ${ }^{18}$ used the $\mathrm{kV} \mathrm{CBCT}$ data for dose calculation by calibration of the CT numbers to ED without any specific cupping correction.

Other groups reported on correction of cupping artifacts mainly to improve image quality. ${ }^{21,22}$ Jarry et al. ${ }^{21}$ corrected the cupping artifact for a kV CBCT bench-top system by using Monte Carlo generated scatter distributions to take into account the contribution of object scatter radiation in the portal images. For kV cone-beam data acquired with a micro-CT scanner, Kachelriess et al. ${ }^{22}$ developed a cupping artifact correction method that passed raw projection image data through a single pixel-independent polynomial before CBCT reconstruction. Their results showed cupping free images after correction, but these authors do not report on the feasibility of their method to correct cupping artifacts of objects with dimensions comparable to human patients. The method and scanner were used for imaging purposes and no efforts were made in quantifying the conversion from CT number to electron density, which is needed in order to use the images for dose calculations.

Correction procedures designed for $\mathrm{kV}$ (cone-beam) CT scans are not guaranteed to be accurate for MV CBCT scans, because MV photon beams have completely different scatter properties compared to the $\mathrm{kV}$ photon beams. In addition, the attenuation of $\mathrm{MV}$ beams is a function of the electron density whereas for $\mathrm{kV}$ photon beams the attenuation also depends on the atomic composition.

The goal of the present study is to use the MV CBCT system for obtaining accurate electron density distributions that can be used for dose calculations. For this purpose, an automated pre-reconstruction cupping correction method is developed that cor- 
rects the cupping artifacts in the MV CBCT images and converts the CT numbers to electron density distributions. Various homogeneous and inhomogeneous phantoms are used to experimentally verify the presented cupping correction algorithm. We compared our method with previously described methods by Kachelriess et al. ${ }^{22}$ and Spies et al. ${ }^{17}$, and differences and similarities with these approaches are discussed. The MV CBCT scans were acquired using a linear accelerator equipped with a commercially available low-dose CBCT acquisition mode and an amorphous silicon EPID that is used in clinical routine for patient imaging.

\section{MATERIALS AND METHODS}

\section{Hardware and operation}

A Siemens Oncor medical linear accelerator with MV CBCT acquisition mode is used in combination with an OptiVue 1000 ST amorphous silicon EPID (Siemens Medical Solutions, Concord, California, USA). The EPID has an active area of $41 \times 41 \mathrm{~cm}^{2}$ with $1024 \times 1024$ pixels and a pixel resolution of $0.4 \mathrm{~mm}$. The Siemens Coherence Therapist Workspace acquisition software was used for the measurement of (cone-beam) portal images.

To acquire an MV CBCT scan of an object, the object is irradiated with the $6 \mathrm{MV}$ treatment beam in a low dose-rate mode with a field size in the isocenter plane of 27.4 $x 27.4 \mathrm{~cm}^{2}$. The total dose at the EPID can be varied from 2 to $60 \mathrm{MUs}$. The source-todetector distance (SDD) is fixed at $145 \mathrm{~cm}$. During MV CBCT acquisition the gantry rotates continuously from 270 을 to 110 o clockwise acquiring 200 portal images with increments of 1 ㅇ. The portal images are automatically corrected for individual pixel sensitivity, variation in intensity along the beam profile, the energy dependent response of the EPID to the non-attenuated beam, dead pixels and dark current. Dead pixels and dark current are corrected by the system interactively. The correction for individual pixel sensitivities, the variations in intensity along the beam profile and the dependence of the response of the EPID on the energetic spectrum of the beam is performed in a single step using flood field images, also referred to as gain images.

The reconstruction of the 2D portal images to a $3 D$ volume is performed using a filtered back-projection method based on the Feldkamp algorithm ${ }^{14}$ implemented in a research version of the cone-beam reconstruction software provided by Siemens (Siemens Medical Solutions, Concord, California, USA). A geometric calibration phantom is used to determine the relation between the position of the voxels of the 3D image and the position of the pixels of the EPID for each gantry angle. The reconstructed volume has dimensions of $25.6 \times 25.6 \times 25.6 \mathrm{~cm}^{3}$ and contains voxels of $1 \times 1 \times$ $1 \mathrm{~mm}^{3}$. The exact calibration procedure and method of $C B$ reconstruction are described in detail by Pouliot et al. ${ }^{23}$

To limit the amount of noise in the MV CBCT images the majority of the scans is performed using the maximum dose of $60 \mathrm{MU}$. To illustrate the feasibility of cupping correction also with low dose $\mathrm{MV} \mathrm{CBCT}$, additional MV CBCT images are made with a total dose of 8 MUs. 


\section{The cupping correction model}

The proposed cupping correction model subtracts the scatter radiation originating from the object, corrects for beam hardening and softening effects and the energy dependent response of the EPID. The correction model consists of 5 steps. First, the raw portal images are filtered with a Perona-Malik diffusion filter and corrected for differences in accelerator output, which is measured with the monitor chamber for each projection. The beam intensity profile is then restored in the portal images of the object. Second, the scatter contribution is predicted and subtracted from the portal images to yield the primary signal. Third, the primary signal is converted to the radiological thickness (i.e. water equivalent thickness or path length) using a mapping function that inherently takes into account beam hardening and softening effects and the energy dependent response of the EPID. With the radiological thickness a new estimate of the scatter is calculated. In step 4, step 2 and 3 are repeated in an iterative manner until convergence of the radiological thickness is achieved. In the fifth step, the final estimate of the radiological thickness is used to calculate the corresponding primary transmission of a mono-energetic beam, which is needed for cone-beam reconstruction. The resulting 200 primary transmission images are used as input for the cone-beam reconstruction algorithm, and the reconstructed $\mathrm{CT}$ numbers are converted to ED.

The correction algorithm is shown schematically in Figure 7.1. The next sections describe these five steps in detail, whereas in a seperate section the derivation of the model parameters is described.

\section{Restoring the beam profile}

The portal images are corrected by the system automatically for the non-flat beam intensity profile by multiplication with a gain image. The first step of the correction model is to restore the beam intensity profile $\mathrm{BP}(\mathrm{x}, \mathrm{y})$ in the measured portal images of the object and of the open beam, which is needed for accurate scatter calculations. This can be described mathematically by

$$
M(x, y, \theta)=I(x, y, \theta) \cdot B P(x, y),
$$

with $(x, y)$ the coordinates in the detector plane, $\theta$ the gantry angle and $I(x, y, \theta)$ the filtered portal images that are corrected for differences in accelerator output by applying the measured signal of the monitor chamber in the linear accelerator head as a scaling factor to the corresponding portal image. $M(x, y, \theta)$ are the portal images in which the beam profile is restored. 


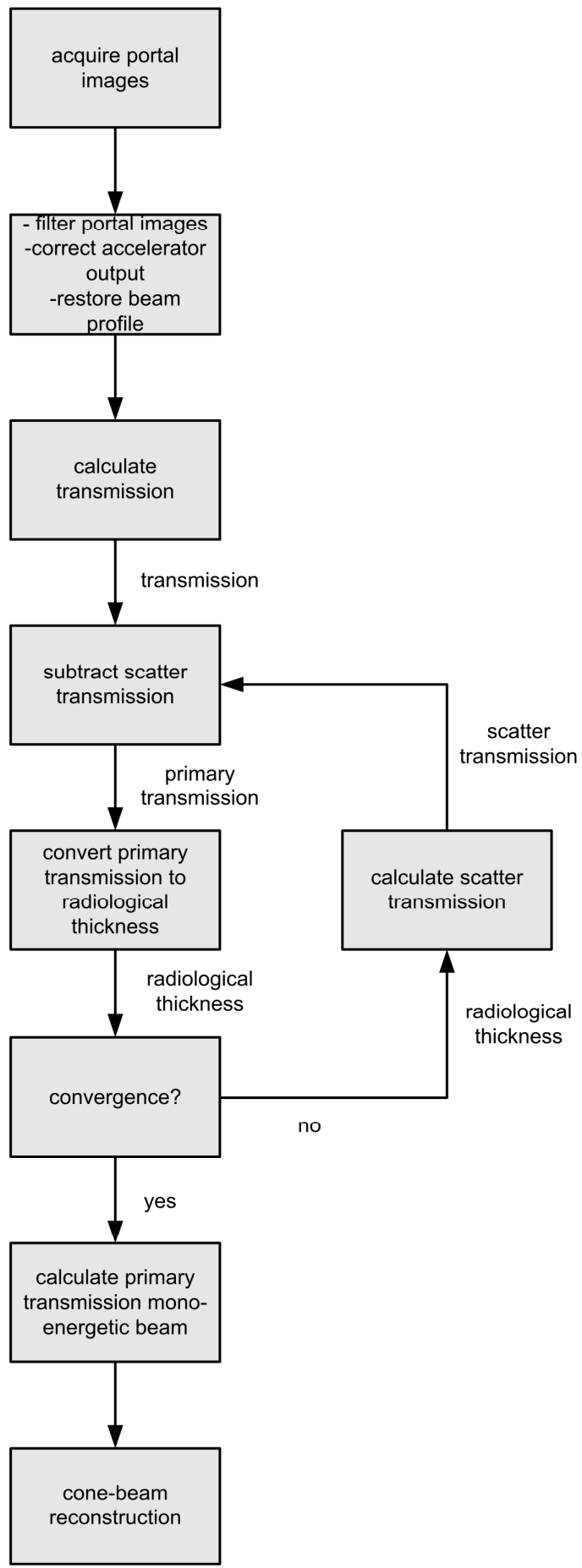

Figure 7.1: Schematic overview of the iterative cupping correction method. 


\section{Removal of scattered transmission}

The total transmission $T(x, y, \theta)$ through an object is the sum of a primary transmission and a scatter component at pixel $(x, y)$ and can be calculated using

$$
T(x, y, \theta)=\frac{M(x, y, \theta)}{M_{\text {Open }}(x, y, \theta)}
$$

with $x$ and $y$ the coordinates at the detector. $M_{\text {open }}(x, y, \theta)$ are the portal images corrected for beam intensity without an object in the beam, also referred to as the open or non-attenuated beam images. The second step of the correction model is to extract the primary transmission $T_{p}\left(x, y, \theta, t_{x, y, \theta}\right)$ from the total transmission by subtracting the scatter component $T_{s}\left(x, y, \theta, t_{\theta}\right)$ which is calculated using the pencil beam concept, ${ }^{24,25}$

$$
\mathbf{T}_{\mathbf{p}}\left(\mathrm{x}, \mathrm{y}, \theta, \mathrm{t}_{\mathrm{x}, \mathrm{y}, \theta}\right)=\mathrm{T}(\mathrm{x}, \mathrm{y}, \theta)-\mathrm{T}_{\mathrm{S}}\left(\mathrm{x}, \mathrm{y}, \theta, \mathbf{t}_{\theta}\right) \text {, }
$$

with $\mathbf{t}_{\theta}$ the distribution of the radiological thickness projected from the focus on the detector plane for gantry angle $\theta$ and $t_{x, y, \theta}$ the projected value of $t_{\theta}$ at the point $(x, y)$. The pencil beam concept describes the scatter originating in the object by the treatment beam as the superposition of the scatter caused by individual pencil beams. The total scattered contribution $T_{s}\left(x, y, \theta, t_{\theta}\right)$ is described by

$$
\mathrm{T}_{S}\left(x, y, \theta, t_{\theta}\right)=\iint_{x^{\prime}, y^{\prime} \in \text { Field }} \frac{M_{\text {Open }}\left(x^{\prime}, y^{\prime}, \theta\right)}{M_{\text {Open }}(x, y, \theta)} K\left(r_{\left(x-x^{\prime}, y-y^{\prime}\right)}, t_{x^{\prime}, y^{\prime}, \theta}\right) d x^{\prime} d y^{\prime},
$$

with $\left(x^{\prime}, y^{\prime}\right)$ the projection of the point where the pencil beam impinges on the object, and $K\left(r_{\left(x-x^{\prime}, y-y^{\prime}\right)}, t_{x^{\prime}, y^{\prime}, \theta}\right)$ are Gaussian scatter kernels ${ }^{25}$, that are only dependent on the water equivalent thickness $t_{x^{\prime}, y^{\prime}, \theta}$ according to

$$
K(r, t)=c_{1}(t) \exp \left(-\left(\frac{r}{c_{2}(t)}\right)^{2}\right)
$$

with $r$ the distance from pixel $(x, y)$ to pixel $\left(x^{\prime}, y^{\prime}\right)$ and $c_{1}(t)$ and $c_{2}(t)$ experimentally determined coefficients (see section on scatter kernel derivation). For scatter estimation, inhomogeneous objects are replaced by homogeneous objects with the same radiological thickness map using the equivalent homogeneous phantom concept. ${ }^{24,25}$

\section{Conversion of transmission to thickness}

For accurate MV CBCT reconstruction, a linear relation is required between the logarithm of the primary transmission and the electron density of the object with thickness, which is the case for the primary transmission of a mono-energetic beam. Due to beam hardening and softening and the energy dependent response of the EPID this condition is not fulfilled for the CBCT acquisition. The degree of beam hardening and softening of a rayline reaching the detector at point $(x, y)$ and the energy dependent response of the EPID at this position only depend on the phantom thickness projected 
on the point $(x, y)$ at the EPID. The third step of the correction model is to extract the radiological thickness $t_{x, y, \theta}$ from the primary transmission images $T_{p}\left(x, y, \theta, t_{x, y, \theta}\right)$. With the mapping function $f$, that uses $M$ sets of coefficients $\alpha_{m}(x, y)$, the radiological thickness $t$ at position $(x, y)$ is related to the $T_{p}\left(x, y, \theta, t_{x, y}, \theta\right)$ according to

$$
T_{p}\left(x, y, \theta, t_{x, y, \theta}\right)=f\left(t_{x, y, \theta},(x, y)\right)=\exp \left(-\sum_{m=1}^{M} \alpha_{m}(x, y) \cdot t_{x, y, \theta}^{m}\right) .
$$

The radiological thickness can then be calculated based on $T_{p}\left(x, y, \theta, t_{x, y, \theta}\right)$ by solving Eq. (7.6) using the inverse of the mapping function $\mathrm{f}^{-1}$

$$
t_{x, y, \theta}=f^{-1}\left(T_{p}\left(x, y, \theta, t_{x, y, \theta}\right),(x, y)\right) .
$$

\section{Iterative correction algorithm}

To calculate the thickness map, the primary transmission must be known, which depends on the scatter contribution (according to Eq. 7.3). However, the scatter contribution depends on its turn on the thickness map. Therefore the thickness map is extracted from the portal images in an iterative manner according to the following steps:

Step (i): Restore the beam profile in the filtered portal images with and without object in the beam that are corrected for differences in accelerator output using Eq. (7.1).

Step (ii): Calculate the transmission using Eq. (7.2).

Step (iii): Calculate the scatter component with the $n^{\text {th }}$ estimate of the thickness, $t^{(n) y, \theta}$ :

$$
T_{S}\left(x, y, \theta, t_{\theta}^{(n)}\right)=\iint_{x^{\prime}, y^{\prime} \in \text { Field }} \frac{M_{\text {Open }}\left(x^{\prime}, y^{\prime}, \theta\right)}{M_{\text {Open }}(x, y, \theta)} K\left(r_{\left(x-x^{\prime}, y-y^{\prime}\right)}, t_{x^{\prime}, y^{\prime}, \theta}^{(n)}\right) d x^{\prime} d y^{\prime} .
$$

Step (iv): Calculate the primary transmission for this thickness estimate:

$$
T_{P}\left(x, y, \theta, t_{x, y, \theta}^{(n)}\right)=T(x, y, \theta)-T_{S}\left(x, y, \theta, t_{\theta}^{(n)}\right) .
$$

Step (v): Calculate a new estimate of the thickness map $t_{x, y, \theta}^{(n)}$ based on the primary transmission

$$
t_{x, y, \theta}^{(n+1)}=f^{-1}\left(T_{p}\left(x, y, \theta, t_{x, y, \theta}^{(n)}\right),(x, y)\right) .
$$

Step (vi): Repeat steps (iii) to (v) until the convergence criterion is reached

$$
\frac{1}{\mathrm{P}} \sum_{\mathrm{x}, \mathrm{y}}\left|\mathrm{t}_{\mathrm{x}, \mathrm{y}, \theta}^{(\mathrm{n}+1)}-\mathrm{t}_{\mathrm{x}, \mathrm{y}, \theta}^{(\mathrm{n})}\right|<\varepsilon,
$$

with $(n)$ denoting the $n^{\text {th }}$ iteration, up to $\mathrm{N}$ iterations are required and $\mathrm{P}$ is the total number of pixels. 
The first estimate of $t_{x, y, \theta}$ in Eq. (7.9) is calculated with Eq. (7.10) using $T$ as a first estimate of $T_{p}$.

Conversion of thickness to the primary transmission of a mono-energetic beam

The cone-beam reconstruction software described in the section hardware and operation and requires the primary transmission of a mono-energetic beam $T_{P, M E}\left(x, y, \theta, t_{x, y, \theta}\right)$ without scatter as input, in order to be able to accurately reconstruct the ED. $T_{P, M E}\left(x, y, \theta, t_{x, y}, \theta\right)$ is calculated using the final estimates of the radiological thickness with

$$
T_{P, M E}\left(x, y, \theta, t_{x, y, \theta}\right)=\exp \left(-\mu \cdot t_{x, y, \theta}\right),
$$

with $\mu$ the attenuation coefficient of a mono-energetic beam with energy $E$ (specified in the section on conversion of thickness to the primary transmission of a monoenergetic beam) in water. In the reconstruction software the log attenuation of the primary transmission is calculated and used in a filtered back-projection method based on the Feldkamp algorithm. ${ }^{14}$

The correction procedure is performed to the 200 portal images of the objects and the set of the $200 T_{M E}\left(x, y, \theta, t_{x, y, \theta}\right)$ images is used as the input for the cone-beam reconstruction software.

\section{Calibration of CT numbers to electron density}

After cone-beam reconstruction the CT numbers are calibrated to electron density. For this purpose a single linear fit is used that converts the reconstructed gray scale values (CT numbers) into electron density values.

\section{Derivation of model parameters}

\section{Raw image filtering and beam profile correction}

To reduce the noise in the final reconstructions the raw portal images are filtered using a diffusion filter ${ }^{26}$ implemented in MevisLab (MevisLab, University of Bremen, Germany) that uses the following equation to filter the images

$$
\frac{\partial \mathrm{l}(\mathrm{x}, \mathrm{y}, \theta)}{\partial \mathrm{s}}=\operatorname{div}\left(\mathrm{g}\left(\left|\nabla \mathrm{I}_{\sigma}(\mathrm{x}, \mathrm{y}, \theta)\right|\right) \nabla \mathrm{l}(\mathrm{x}, \mathrm{y}, \theta)\right)
$$

with $I(x, y, \theta)$ the image, $I_{\sigma}(x, y, \theta)$ is the convolution of $I(x, y, \theta)$ by a Gaussian with standard deviation $\sigma$ and $g$ the Perona-Malik function

$$
g\left(\left|\nabla \mathrm{I}_{\sigma}(\mathrm{x}, \mathrm{y}, \theta)\right|\right)=\frac{1}{1+\left(\frac{\left|\nabla \mathrm{I}_{\sigma}(\mathrm{x}, \mathrm{y}, \theta)\right|}{\kappa}\right)^{2}}
$$


with $\mathrm{k}$ the edge parameter. Eq. (7.13) is solved using a semi-implicit scheme with 2 iterations, a step-size $\mathrm{s}$ of $5, \sigma$ is equal to 2 times the pixel spacing and a $\mathrm{k}$ of 10 . The standard functions implemented in MevisLab were used to perform all filtering operations, e.g. convolutions and the calculation of gradients and derivatives.

The incident beam profile was estimated from a dose profile measured with an ionization chamber in a water phantom at a depth of $14 \mathrm{~mm}$ at a source-to-surface distance of $90 \mathrm{~cm}$ and a field size of $40 \times 40 \mathrm{~cm}^{2}$ in the plane through the isocenter. The measured profile was rescaled to the detector plane by correcting for the divergence of the beam.

\section{Scatter kernel derivation}

The coefficients of the pencil beam kernels $c_{1}(t)$ and $c_{2}(t)$ are derived using on-axis transmission measurements with polystyrene slab phantoms with known thickness and an ED of 0.97 . The thickness of the treatment couch is taken into account by adding $1.5 \mathrm{~cm}$ to the thickness of the slab phantoms stacks. The phantoms are positioned symmetrically with regard to the horizontal plane through the isocenter (i.e. a distance of $100 \mathrm{~cm}$ from the focus of the linac). The phantoms are irradiated with different field sizes, $6 \times 6,10 \times 10,15 \times 15,20 \times 20$ and $25 \times 25 \mathrm{~cm}^{2}$, centered around the isocenter with the $6 \mathrm{MV}$ beam using a total dose of $100 \mathrm{MUs}$. Because the primary transmission is independent of field size, the difference in on-axis transmission values is used to fit both primary transmission and the coefficients of the scatter kernels for phantom thickness ranging between 3.4 and $36.7 \mathrm{~cm}$. A detailed description of this fit procedure is described elsewhere. ${ }^{25}$

\section{Transmission to thickness mapping function}

The transmission to thickness mapping function $f$ is derived using the transmission measurements with the $25 \times 25 \mathrm{~cm}^{2}$ field size, that were used to derive the scatter kernels. The measurements are extrapolated to cover the entire detector plane. The scatter component $T_{s}(x, y, \theta, t)$ of the slab phantoms is calculated using Eq. (7.8) and subtracted from the measured transmission to obtain the primary transmission $T_{p}\left(x, y, \theta, t_{x, y, \theta}\right)$. The coefficients $\alpha_{m}$ of the transmission to thickness mapping function are derived for every point $(x, y)$ in the portal image by fitting the primary transmission values to the phantom thicknesses using a $3^{\text {rd }}$ degree polynomial $(M=3)$. The inverse of the mapping function $\mathrm{f}$ for $\mathrm{M}=3$ generally has 3 solutions. The algorithm automatically selects the thickness value between 0 and $50 \mathrm{~cm}$.

\section{Iterative correction}

The convergence criterion $\varepsilon$ was set to $0.03 \mathrm{~cm}$ and as a result the number of iterations $N$ are different per portal image. We investigated the effect of varying the number of iterations on the final solution. Simulations are performed with a fixed number of either $0,1,2,3,4,5,10,15$ or 20 iterations. To gain more insight in the influence of the various steps of the correction procedure the results without applying a scatter 
correction are analyzed (i.e. measured transmission is directly converted to thickness using the mapping function) as well as the results when only the scatter correction in the iterative loop is applied without using the mapping function to calculate the radiological thickness.

\section{Conversion of thickness to the primary transmission of a mono-energetic beam}

The attenuation coefficient $\mu$ of water in Eq. (7.12) for a $6 \mathrm{MV}$ beam was set to 0.043 $\mathrm{cm}^{-1}$ corresponding to a mono-energetic photon beam of $2.7 \mathrm{MeV}$. However, the accuracy of the method does not depend on the exact value of $\mu$ because the resulting deviation in $T_{P, M E}$ is canceled out by the subsequent calibration from CT number to ED.

\section{CT number to electron density calibration}

The calibration of the reconstructed CT numbers to electron density is performed using a simple 2 point calibration curve. The first point is the average CT number within the corrected $\mathrm{CB}$ reconstruction of a $20 \mathrm{~cm}$ diameter water cylinder and the second point is the CT number of the air outside the phantom. A linear fit is used for this calibration.

\section{Verification of the model}

In order to verify the accuracy of our model in terms of ED calibration and dose calculations, MV CBCT images are made for a large number of phantoms and reconstructed ED distributions are used for dose calculations. The next sections describe the phantoms that were used and the dose calculations that were performed for this verification.

\section{Homogeneous phantoms}

First, two PMMA (polymethyl methacrylate) cylinders filled with water were used. The first cylinder has a radius $R$ of $10 \mathrm{~cm}$, a length of $30 \mathrm{~cm}$ and a wall thickness of $5 \mathrm{~mm}$. The second cylinder has a radius of $5 \mathrm{~cm}$, a length of $30 \mathrm{~cm}$ and a wall thickness of 3 $\mathrm{mm}$. The cylinders are referred to as the large and small cylinder, respectively. An IMRT head-and-neck phantom (CIRS, Norfolk, Virginia, USA) was used. This phantom is cylindrically shaped with a radius of $8 \mathrm{~cm}$ and composed of a material with water equivalent ED. The cylinder contains 5 cavities that can accommodate rod inserts. For the homogenous IMRT phantom water equivalent inserts were used. All cylinders are positioned on the treatment table with the central axis coinciding with the axis of rotation of the linac ( $y$-axis). The $z$-axis is the vertical axis and the $x$-axis the crossplane axis. 


\section{Inhomogeneous phantoms}

Various inhomogeneous phantoms were used. The IMRT head-and-neck phantom was used with one insert filled using bone-equivalent material ( $E D=1.51$ ).

Phantom A was a simple slab phantom with dimensions $30 \times 14.5 \times 14.8 \mathrm{~cm}^{3}$ containing two low-density $(E D=0.19)$ regions of cork of $30 \times 14.5 \times 4.2 \mathrm{~cm}^{3}$ embedded between layers of polystyrene $(E D=0.97)$.

Phantom B consisted of two slabs of polystyrene with a slab of cork and with on top the small $(R=5 \mathrm{~cm})$ homogeneous water cylinder.

Phantom $C$ was a PMMA cylinder with a radius of $10 \mathrm{~cm}$ filled with water that contained cylindrical inserts with different electron densities. The ED values of these inserts relative to water (1.00) were based on muscle (1.03), fat (0.91), cortical bone (1.68), breast (0.96), inner bone (1.14) and lung (0.32). The 6 inserts are placed inside the three cavities of the phantom, in such a way that each cavity contains two inserts positioned behind one another.

An anthropomorphic Rando head phantom was used to simulate an actual patient geometry including bone, air and soft tissue equivalent regions. A kV planning CT scan was made (CT Open, Siemens Medical Solutions, Erlangen, Germany) and the Hounsfield Units were converted to ED using our clinically applied conversion table. A transversal slice of the $\mathrm{kV}$ planning $\mathrm{CT}$ scan was compared to corresponding images of the uncorrected and corrected MV CBCT scans.

\section{Dose calculations}

Dose calculations were performed to investigate the influence of the cupping artifact and the ED conversion in terms of calculated dose. The small and large homogeneous cylinder and the homogeneous IMRT phantom were used. Corrected and uncorrected CBCT images of these phantoms were imported in the treatment planning system XiO (version 4.3.3, CMS, St. Louis, MO, USA). Dose calculations were performed using the Multi-Grid superposition algorithm and a $2 \mathrm{~mm}$ calculation grid. The algorithm implemented in the planning system calculates the dose distribution $D(\vec{r})$ at the position $\vec{r}$ as the superposition of the incident energy fluence $\Psi_{0}$, that is corrected for the attenuation along the raylines using the attenuation coefficients distribution $\mu / \rho(\vec{r})$, with a dose deposition kernel $k\left(\vec{r} ; \vec{r}^{\prime}\right)$

$$
D(\vec{r})=\int \Psi_{0}\left(\vec{r}^{\prime}\right) \frac{\mu}{\rho}\left(\vec{r}^{\prime}\right) e^{-\int_{0}^{r^{\prime}} \mu\left(\vec{r}^{\prime}\right) d \vec{r}^{\prime}} \cdot k\left(\vec{r} ; \vec{r}^{\prime}\right) d \vec{r}^{\prime} .
$$

Here it is clearly shown that the correct attenuation coefficient distribution is necessary for accurate dose distribution calculations. Dose calculations were performed for a $5 \times 5 \mathrm{~cm}^{2} 6 \mathrm{MV}$ beam with $100 \mathrm{MUs}$ with the central axis coinciding with the $\mathrm{x}$-axis of the system using the three homogeneous cylindrical phantoms. MV CBCT images of the cylinders with the ED forced manually to 1.0 inside the cylinder are used as a reference. The dose distributions were analyzed using percentage depth dose curves 
and the absolute dose values at the center and $2 \mathrm{~cm}$ upstream of the exit surface of the phantom are reported.

\section{Comparison with other methods}

The presented method is compared with a method described by Kachelriess et al. ${ }^{22}$ who corrected for cupping artifacts by passing the log attenuation of the portal images through a single fourth order polynomial before cone-beam reconstruction. The coefficients of the polynomial are derived by reconstructing a set of monomial basis images from the projections of a homogenous cylinder. Then the set of coefficients is found by minimizing the linear combination of the reconstructed basis images with the coefficients and a template image that represents the desired ideal image without cupping. We implemented this method with a polynomial degree of order 4 and derived the coefficients using the IMRT phantom (radius $=8 \mathrm{~cm}$ ) with only water equivalent inserts. The method was tested on the Rando head phantom and compared with the images obtained using a kV planning CT scan. After the cupping correction the images were calibrated to ED using a scale factor that was derived by setting the ED equal to 1 in a water equivalent region in the center of the phantom.

We also partly implemented the method of Spies et al. ${ }^{17}$ who used a combination of Monte Carlo derived scatter kernels with a quadratic correction for beam hardening, i.e. $M=2$ for the transmission to thickness mapping function. The differences between the methods are analyzed for the large and small water cylinder. We did not have the availability of a Monte Carlo code to calculate these scatter kernels but instead used our measurement derived kernels.

\section{RESULTS}

\section{The cupping correction model}

The pencil beam scatter kernels are fitted with high accuracy; the maximum difference between the fitted and measured transmission was smaller than $0.3 \%$. The coefficients of the kernels are shown in Table 7.1. With increasing phantom thickness, the kernels are more sharply peaked, reflected by the decreased width $c_{2}(t)$ of the kernels, while

Table 7.1: Fitted scatter kernel coefficients $c_{1}$ and $c_{2}$.

\begin{tabular}{|c|c|c|c|c|c|}
\hline $\begin{array}{c}\mathbf{t} \\
{[\mathbf{c m}]}\end{array}$ & $\begin{array}{c}\boldsymbol{c}_{\mathbf{1}} \\
{\left[\mathbf{1 0 ^ { - 5 }} \mathbf{c m}^{-\mathbf{2}}\right]}\end{array}$ & $\begin{array}{c}\boldsymbol{c}_{\mathbf{2}} \\
{[\mathbf{c m}]}\end{array}$ & $\begin{array}{c}\mathbf{t} \\
{[\mathbf{c m}]}\end{array}$ & $\begin{array}{c}\boldsymbol{c}_{\mathbf{1}} \\
{\left[\mathbf{1 0} \mathbf{1}^{-\mathbf{2}} \mathbf{c m}^{-\mathbf{2}}\right]}\end{array}$ & $\begin{array}{c}\boldsymbol{c}_{\mathbf{2}} \\
{[\mathbf{c m}]}\end{array}$ \\
\hline 0 & 0.0 & 0.0 & & & \\
3.4 & 2.76 & 42.3 & 16.9 & 9.63 & 69.5 \\
5.0 & 3.94 & 102 & 20.3 & 10.5 & 48.5 \\
6.7 & 5.01 & 361 & 22.2 & 10.7 & 43.7 \\
9.0 & 6.50 & 2061 & 25.6 & 10.9 & 38.7 \\
11.4 & 7.73 & 597 & 28.9 & 10.8 & 36.3 \\
13.2 & 8.40 & 153 & 32.5 & 10.6 & 33.2 \\
14.9 & 9.15 & 74.8 & 36.7 & 10.3 & 30.8 \\
\hline
\end{tabular}


the on-axis absolute scatter is increased, indicated by parameter $c_{1}(t)$.

The combined effect of the energy dependent response of the EPID with beam hardening and softening on the primary transmission is illustrated in Figure 7.2. The left figure illustrates the difference in on-axis primary transmission between the polyenergetic clinical treatment beam and a mono-energetic beam. The right figure shows
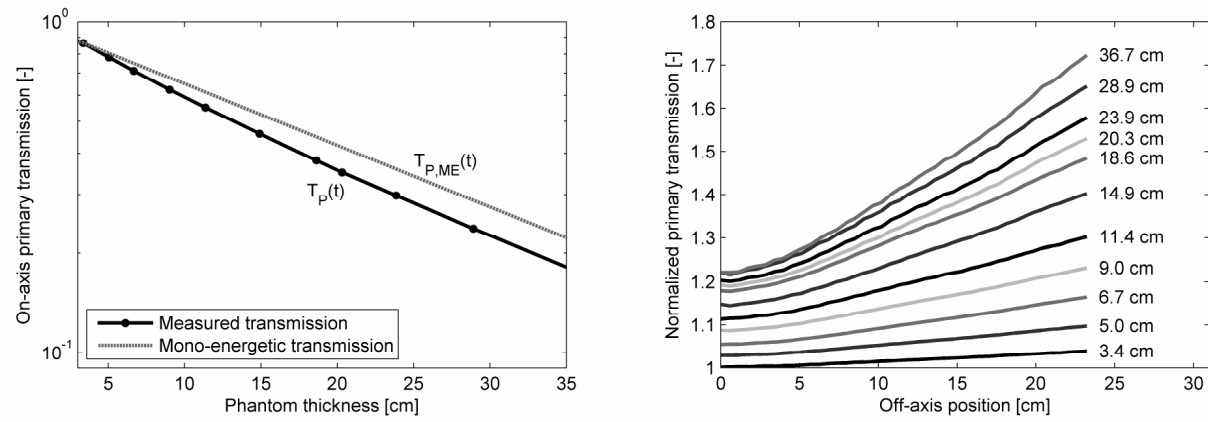

Figure 7.2: The left plot shows the measured on-axis transmission for various phantom thicknesses together with the calculated transmission of a mono-energetic photon beam with a $\mu$ of $0.043 \mathrm{~cm}^{-1}$. The right plot shows the primary transmission as a function of the off-axis position for different phantom thicknesses, normalized to the on-axis primary transmission of a monoenergetic beam. The transmission values are used to determine the $\alpha_{m}$ coefficients needed to convert primary transmission to thickness.
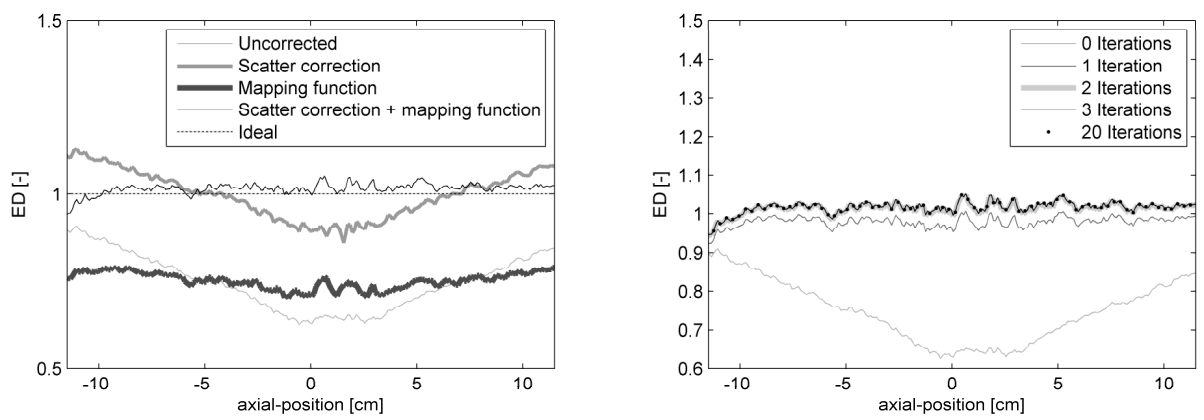

Figure 7.3: The left plot shows the ED along the radial axis of the water cylinder with a radius of $10 \mathrm{~cm}$ for the various corrections: portal images only corrected for scatter (thick gray curve), only the transmission to thickness mapping function applied (thick black curve) and both the scatter correction and the mapping function applied (thin black curve). In addition the uncorrected (thin gray curve) and the real ED (dotted line) are shown. The right plot shows the ED along the radial axis of the same phantom for a varying number of iterations in the iterative correction model. The ED converges rapidly after two iterations. 
the primary transmission as a function of the off-axis distance for different phantom thicknesses, normalized to the (on-axis) primary transmission of a mono-energetic beam. The maximum difference between on- and off-axis locations in terms of the primary transmission can be up to $40 \%$ for large phantom thicknesses. The on-axis parameters $\alpha_{\mathrm{m}}$ were $0.051 \mathrm{~cm}^{-1}$ (off-axis values range from 0.050 to $0.070 \mathrm{~cm}^{-1}$ ), $1.17 \cdot 10^{-4} \mathrm{~cm}^{-2}$ (off-axis range $-1.0 \cdot 10^{-3}$ to $+1.5 \cdot 10^{-4} \mathrm{~cm}^{-2}$ ) and $-5.43 \cdot 10^{-6} \mathrm{~cm}^{-3}$ (off-axis range $-7.6 \cdot 10^{-6}$ to $+1.5 \cdot 10^{-5} \mathrm{~cm}^{-3}$ ) for $\mathrm{m}=1,2$ and 3 , respectively.

The influence of the scatter correction and of the conversion of the transmission to radiological thickness on the final solution was analyzed. The left plot in Figure 7.3 shows ED profiles along the radial axis of the large water cylinder $(R=10 \mathrm{~cm})$. The effect of the scatter correction on the ED is similar to the effect of applying a constant offset to the ED in the entire phantom, which results in a mean ED that is equivalent to the real ED but the cupping artifact is still present. The ED without the scatter correction shows hardly any cupping but a systematic lower ED of approximately 0.25.

The influence of the number of iterations $\mathrm{N}$ was also analyzed. The iterative cupping correction model rapidly converges after 2 to 3 iterations to the final solution. This is shown in terms of the ED in the right plot of Figure 7.3 for a fixed number of

Table 7.2: ED values of the phantoms and materials of the uncorrected and corrected MV CBCT scans.

\begin{tabular}{|c|c|c|c|c|}
\hline Phantom & Material & $\begin{array}{c}\text { ED } \\
\text { relative to } \\
\text { water } \\
{[-]}\end{array}$ & $\begin{array}{c}\text { ED } \\
\text { uncorrected } \\
\text { MV CBCT scans } \\
\text { mean } \pm \text { SD }[-]\end{array}$ & $\begin{array}{c}\text { ED } \\
\text { corrected } \\
\text { MV CBCT scans } \\
\text { mean } \pm \text { SD }[-]\end{array}$ \\
\hline $\begin{array}{l}\text { Water cylinder } \\
(\mathrm{R}=10 \mathrm{~cm})\end{array}$ & Water & 1.00 & $0.86 \pm 0.09$ & $1.00 \pm 0.03$ \\
\hline $\begin{array}{l}\text { Water cylinder } \\
(\mathrm{R}=5 \mathrm{~cm})\end{array}$ & Water & 1.00 & $1.01 \pm 0.05$ & $1.01 \pm 0.03$ \\
\hline $\begin{array}{l}\text { Water cylinder } \\
(\mathrm{R}=10 \mathrm{~cm}) . \\
\text { Total dose } 8 \mathrm{MU}\end{array}$ & Water & 1.00 & $0.84 \pm 0.11$ & $0.99 \pm 0.04$ \\
\hline \multirow[t]{2}{*}{ Phantom A } & Polystyrene & 0.97 & $0.94 \pm 0.06$ & $0.95 \pm 0.03$ \\
\hline & Cork & 0.19 & $0.17 \pm 0.03$ & $0.21 \pm 0.02$ \\
\hline \multirow[t]{3}{*}{ Phantom B } & Water & 1.00 & $0.92 \pm 0.06$ & $0.97 \pm 0.03$ \\
\hline & Polystyrene & 0.97 & $0.94 \pm 0.05$ & $0.97 \pm 0.03$ \\
\hline & Cork & 0.19 & $0.15 \pm 0.04$ & $0.21 \pm 0.03$ \\
\hline \multirow[t]{7}{*}{ Phantom C } & Lung & 0.32 & $0.28 \pm 0.04$ & $0.44 \pm 0.04$ \\
\hline & Fat & 0.91 & $0.79 \pm 0.03$ & $0.93 \pm 0.03$ \\
\hline & Breast & 0.96 & $0.94 \pm 0.03$ & $0.99 \pm 0.02$ \\
\hline & Water & 1.00 & $0.87 \pm 0.07$ & $1.01 \pm 0.03$ \\
\hline & Muscle & 1.03 & $0.76 \pm 0.05$ & $1.05 \pm 0.04$ \\
\hline & Inner bone & 1.14 & $0.93 \pm 0.04$ & $1.13 \pm 0.03$ \\
\hline & Cortical bone & 1.68 & $1.49 \pm 0.05$ & $1.64 \pm 0.03$ \\
\hline \multirow[t]{2}{*}{ IMRT phantom } & Water & 1.00 & $0.93 \pm 0.05$ & $0.95 \pm 0.03$ \\
\hline & Bone & 1.51 & $1.21 \pm 0.03$ & $1.36 \pm 0.04$ \\
\hline IMRT phantom & Water & 1.00 & $0.93 \pm 0.06$ & $0.96 \pm 0.02$ \\
\hline
\end{tabular}


iterations varying from 0 to 3 . Increasing the number of iterations to as much as 20 does not affect the results. For $\mathrm{N}>3$ the mean and the standard deviation (SD) of the differences in ED in the entire phantom between two subsequent iterations are below 0.001 ED units.

\section{Model verification}

\section{Homogeneous phantoms}

The correction model is applied to the portal images of a PMMA cylinder filled with water with a radius of $10 \mathrm{~cm}$. Figure 7.4 shows on the first and second row gray scale images of an axial and sagittal slice through the isocenter of an uncorrected and a corrected MV CBCT scan. The window levels are set to the mean ED \pm 2 standard deviations (SD). This allows a visual inspection of the homogeneity of the reconstructed images. If artifacts are removed the window level is determined solely by the level of noise. The cupping artifact is clearly visible in the uncorrected images (the ED is $32 \%$ smaller in the center than near the wall of the cylinder) but when the cupping correction method is applied the variations in ED due to the cupping are smaller than the level of noise. The results expressed as a mean \pm 1 SD of the ED in the entire phantom are $0.86 \pm 0.09$ if the images are not corrected and $1.00 \pm 0.03$ if the correction model is applied (Table 7.2). The two bottom rows of Figure 7.4 shows the corresponding figures for a water cylinder with a radius of $5 \mathrm{~cm}$. Due to the smaller dimensions of this phantom the cupping is less pronounced than in the larger cylinder, but it is still clearly visible. Applying the correction model results in cupping free images (ED of $1.01 \pm 0.03$ compared to $1.01 \pm 0.05$ without cupping correction). Profiles of the ED in the two cylinders along the $x$ - and $y$-axis are shown in Figure 7.5. The cupping artifact is corrected in all 3 directions for both phantoms. The ED peaks near the wall of the phantoms are caused by the increased ED of the PMMA (ED 1.15) wall compared to water.

In addition the cupping correction method was applied to the cylindrical IMRT phantom with a radius of $8 \mathrm{~cm}$ containing only water equivalent inserts. The cupping artifact of 0.065 ED units (1 SD), present in the uncorrected MV CBCT scan, was removed by the correction method yielding a SD of 0.021 ED units. The mean ED after correction was 0.04 ED units lower than the real ED compared to 0.07 ED units with the uncorrected MV CBCT scan.

To verify whether the correction model is capable of correcting cupping artifacts of low-dose MV CBCT images, a MV CBCT scan of the cylinder with a radius of $10 \mathrm{~cm}$ with a total dose of $8 \mathrm{MUs}$ was analyzed. Correction and $C B$ reconstruction of the portal images resulted also in cupping free images (Table 7.2). The low-dose mode resulted in a lower signal-to-noise ratio, reflected by a slightly increased SD of 0.04 ED units compared to 0.03 for the $60 \mathrm{MU}$ MV CBCT scan. 


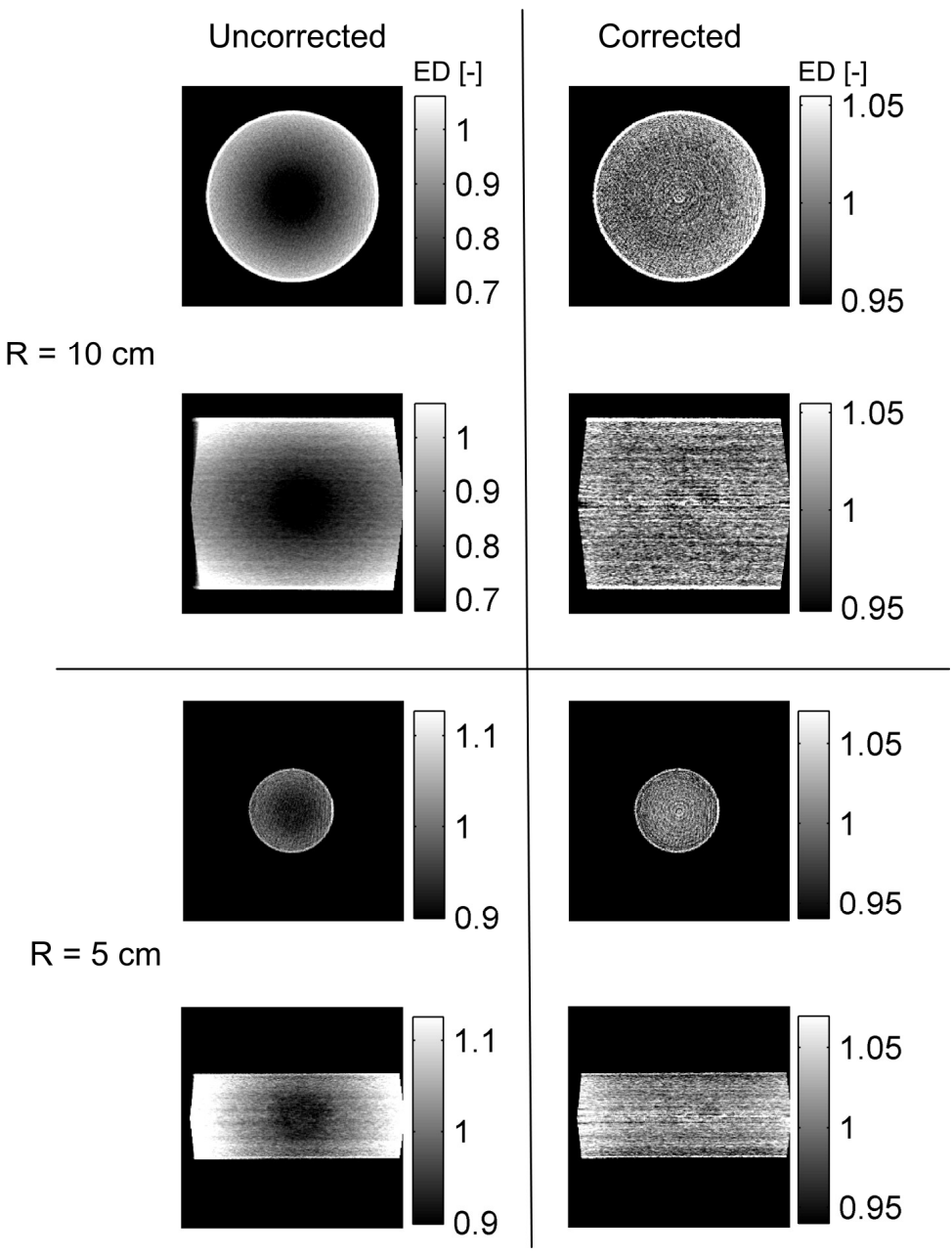

Figure 7.4: The left and right column represent the uncorrected and corrected MV CBCT scans, respectively, of two PMMA cylinders filled with water with radii of 5 and $10 \mathrm{~cm}$. The first and third row show the central axial slices of the MV CBCT scans and the second and fourth row show sagittal slices. The window levels are set to the mean ED in the phantoms $\pm 2 S D$. In the corrected images the window level is determined by the level of noise, which indicates that no residual cupping is present. 


\section{Inhomogeneous phantoms}

The accuracy of the cupping correction method was also assessed using five inhomogeneous objects. For the first object, Phantom A, a schematic representation is shown in the top left figure in Figure 7.6. The ED of the cork (0.19) and polystyrene (0.97) were derived from the $\mathrm{kV}$ planning CT scan. ED profiles along the white dotted line are shown in the top right figure. Due to the cupping artifact the ED is decreased in the polystyrene layer in the middle and in the cork regions, but this was corrected by the cupping correction method. The bottom row shows absolute difference images between the reconstructed ED in the MV CBCT scans and the real ED. The black and white areas indicate regions were the ED is under- or overestimated and correctly reconstructed, respectively. These images confirm that the cupping artifact was corrected using the method proposed here. The mean and SD of the ED in the cork and polystyrene regions are given in Table 7.2.

The second inhomogeneous phantom (Phantom B) consisted of slabs of polystyrene and cork with on top a cylindrical phantom filled with water with a diameter of 10 $\mathrm{cm}$ (Figure 7.7 top left). The ED profiles and the absolute difference images show that the ED was accurately reconstructed after the cupping correction method was applied. Figure 7.8 shows histograms of the ED distributions that confirm the clear reduction of
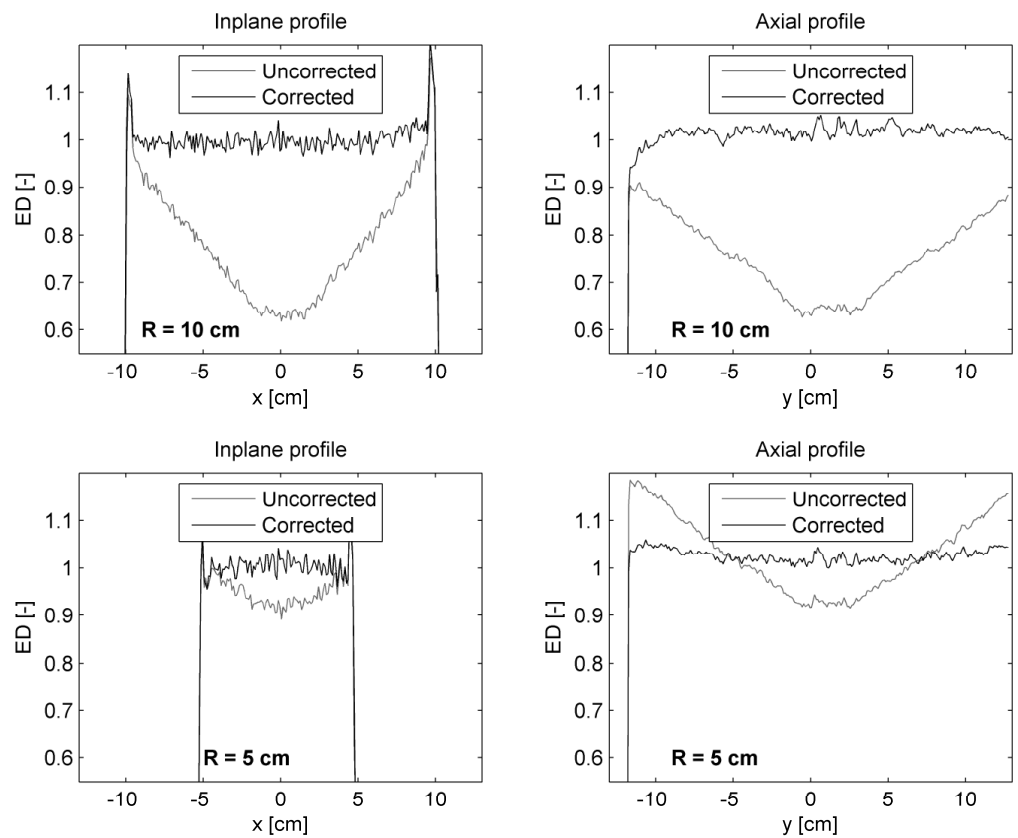

Figure 7.5: Line profiles of the ED along the $x$-and $y$-axis in the water cylinders with radii of 10 and $5 \mathrm{~cm}$ respectively. The cupping artifacts are corrected in three dimensions. The peaks near the walls of the cylinder are caused by the higher ED of the PMMA wall. 

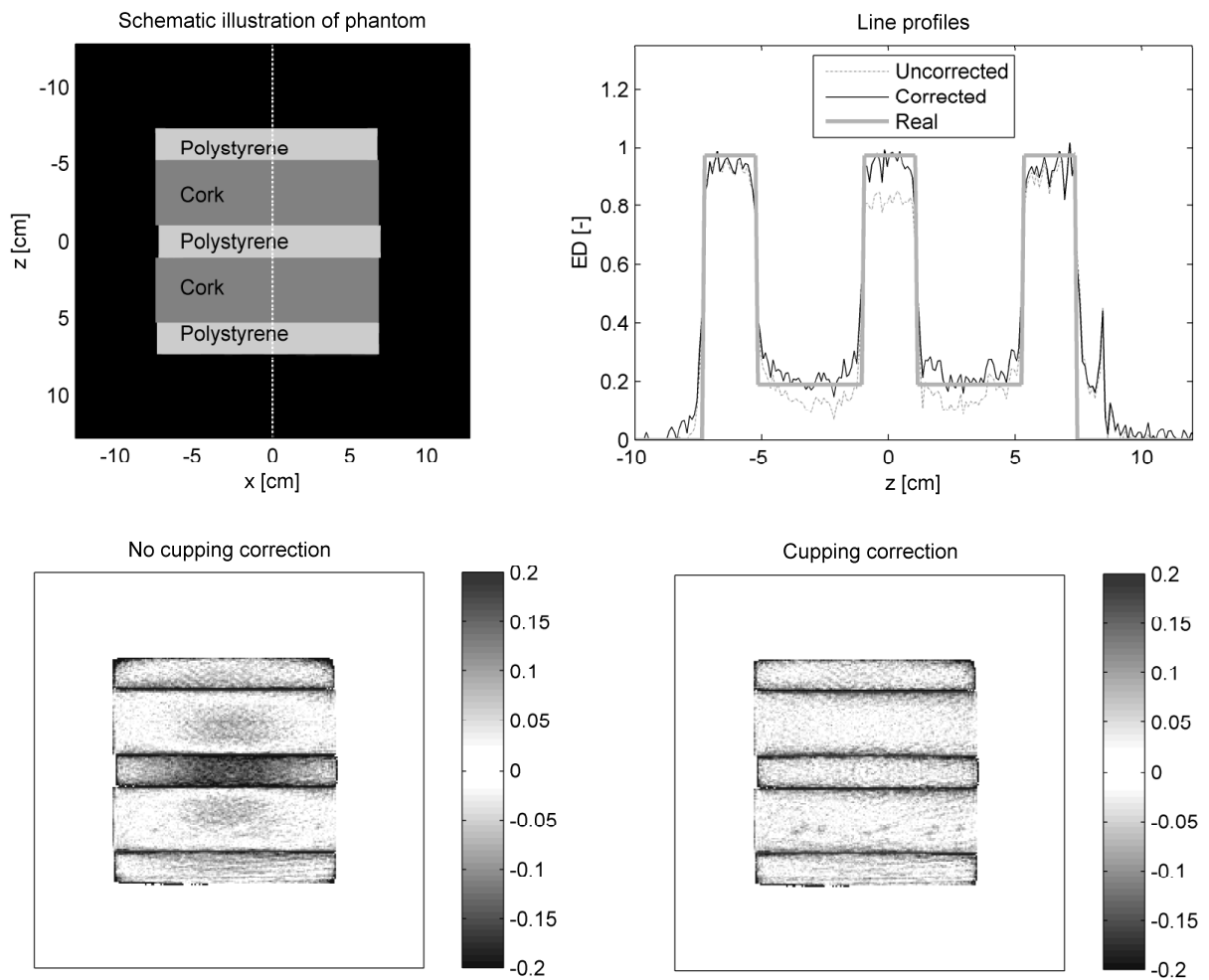

Figure 7.6: Illustration of the corrected and uncorrected MV CBCT scans of Phantom A. The top left figure shows a schematic illustration. The top right figure shows ED profiles along the line $x=$ $0 \mathrm{~cm}$ indicated in the top left figure. The thick gray line represents the real ED. The bottom figures represent absolute difference images of the uncorrected and corrected MV CBCT scan with the ideal image. The cupping artifact is corrected by the proposed cupping correction method.

the cupping artifact indicated by the smaller width of the distributions after correction than without correction.

The third inhomogeneous phantom (Phantom C) was a water cylinder with a radius of $10 \mathrm{~cm}$ that contained cylindrical inserts of varying ED. If uncorrected, the ED is underestimated up to $0.30 \mathrm{ED}$ units in the water region and is systematically more than 0.19 ED units too low in the inserts with ED values corresponding to muscle, inner bone and cortical bone. Figure 7.9 shows line profiles and absolute difference images. If the cupping correction method is applied the ED deviated at maximum 0.04 ED units from the real ED in water and in all inserts except in lung (Table 7.2). 

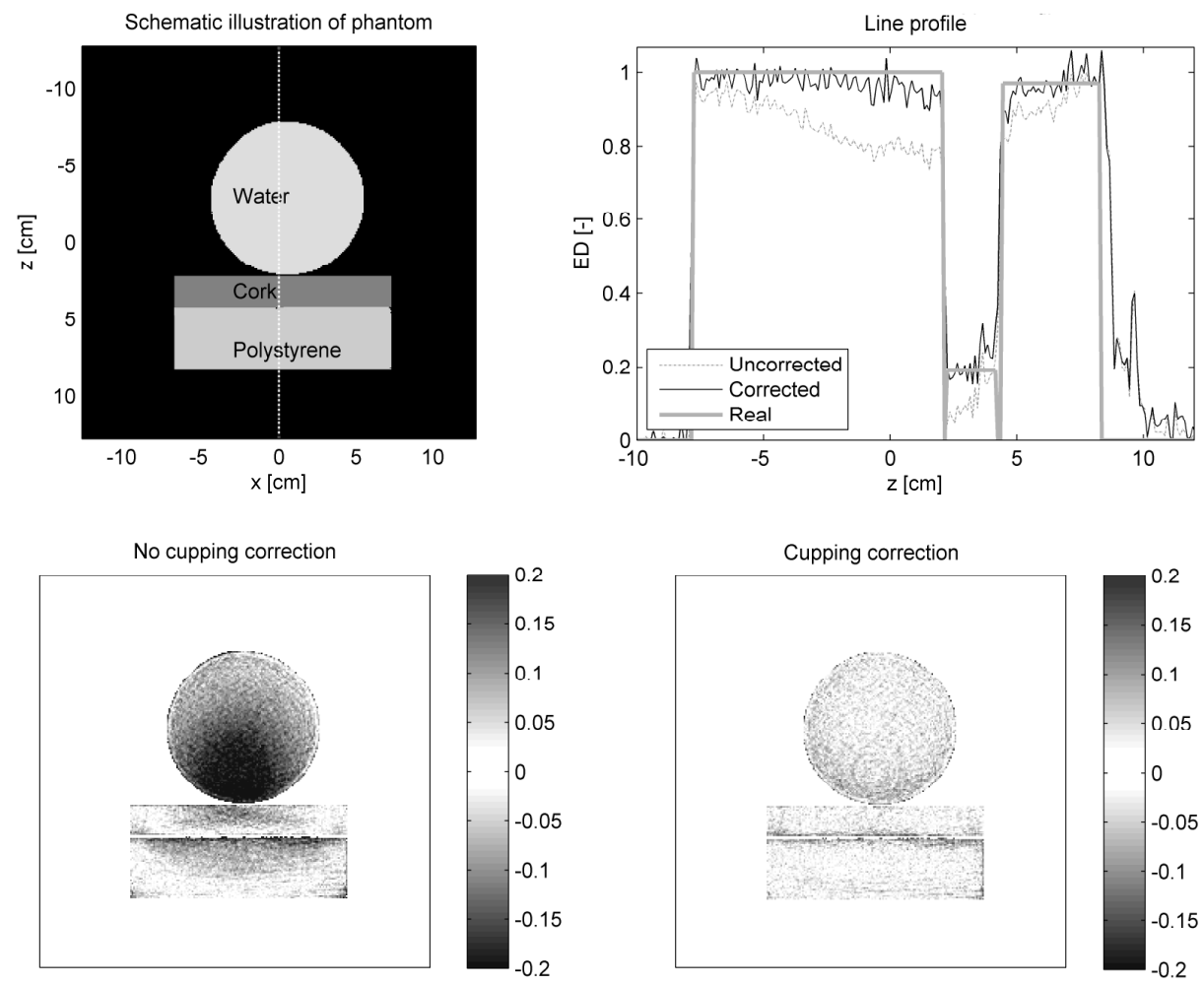

Figure 7.7: Results for the corrected and uncorrected MV CBCT scans of Phantom B.
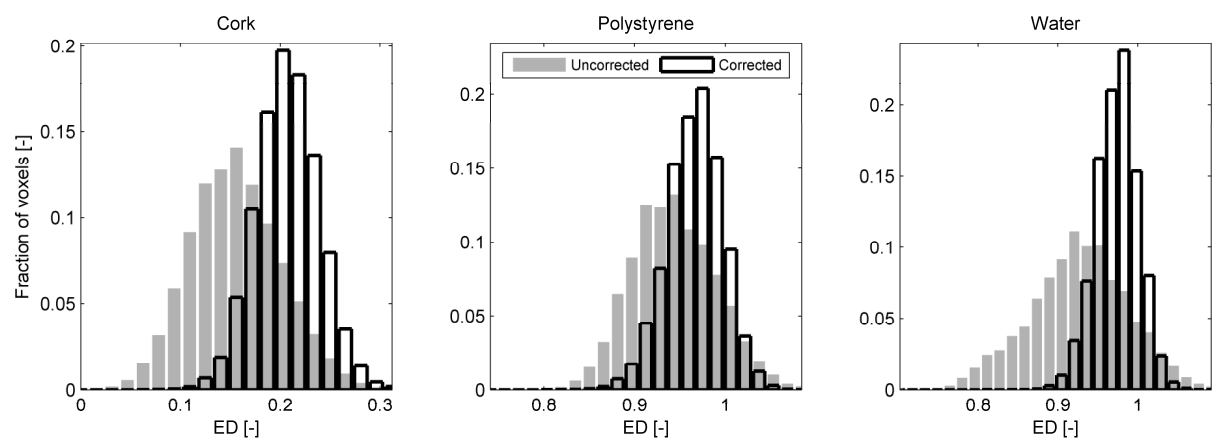

Figure 7.8: ED distributions of the cork, polystyrene and water regions in Phantom B. The solid and open histograms represent the uncorrected and corrected MV CBCT scans, respectively. The distributions of the corrected scans are more sharply peaked than the uncorrected scans indicating that the cupping artifacts have decreased. 

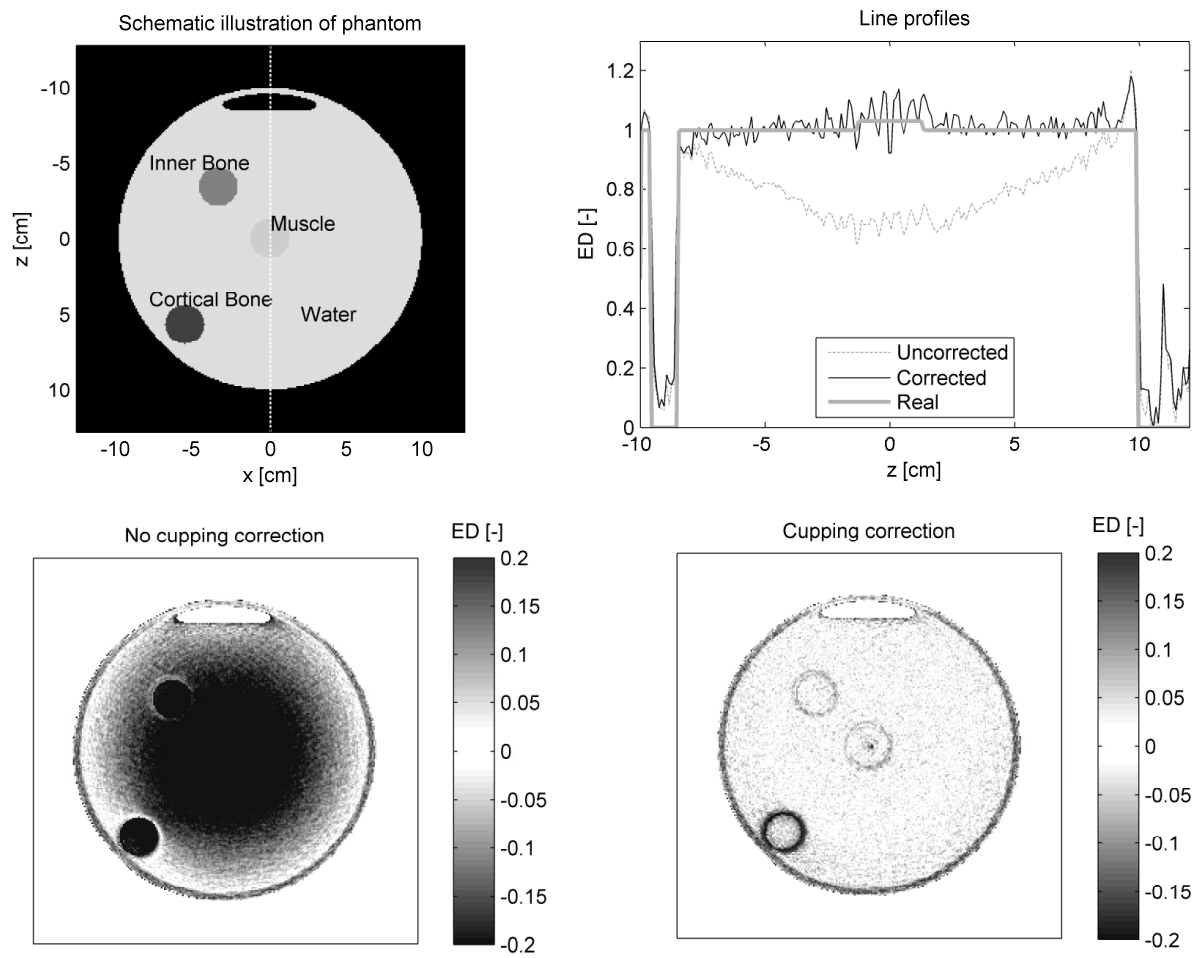

Figure 7.9: Results for the corrected and uncorrected MV CBCT scans of Phantom C.

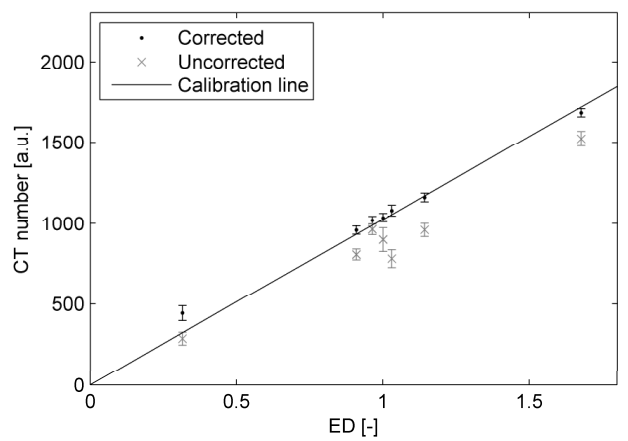

Figure 7.10: Reconstructed CT numbers as function of the ED of the inserts and water of Phantom $C$. The dots and crosses represent the mean ED of the corrected and uncorrected MV CBCT scans, respectively. The error bars represent 1 SD. The calibration line is also shown. 

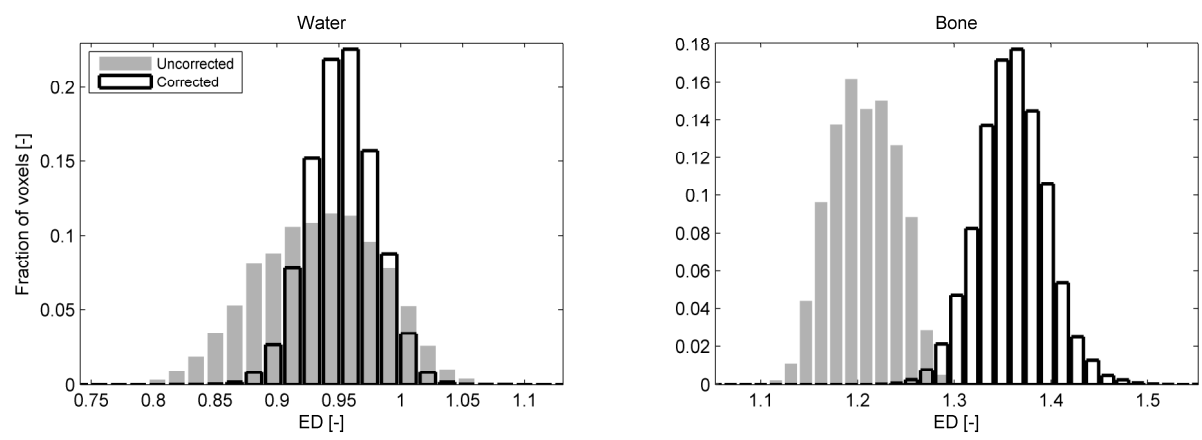

Figure 7.11: ED distributions of the water and bone equivalent regions in de IMRT phantom. The solid and open histograms represent the uncorrected and corrected MV CBCT scans, respectively.

The corrected MV CBCT scan of Phantom $\mathrm{C}$ was used to verify whether an accurate calibration from CT number to ED can be performed with a linear curve. Figure 7.10 shows the $\mathrm{CT}$ numbers of the inserts as function of the real ED together with the calibration line. All inserts except lung are within 0.04 ED units from the calibration line. Without cupping correction it is not possible to obtain a linear relation between CT number and ED with the same accuracy.

Figure 7.11 shows a histogram of the ED distributions of the IMRT phantom with 4 water equivalent inserts and one bone insert ( $E D=1.51)$. The reduction of the cupping artifact is clearly visible. By applying the cupping correction method the mean ED values are shifted from 0.93 to 0.95 and from 1.21 to 1.36 for water and the bone insert, respectively.

The results of applying the cupping correction method to the Rando head phantom are shown in Figure 7.12. A transversal kV planning CT slice is compared with the corresponding uncorrected and corrected MV CBCT slices. If the image quality of the MV CBCT is visually compared with the $\mathrm{kV}$ planning CT image, a lower signal-to-noise ratio is observed. Also visible are streak artifacts in the $\mathrm{kV}$ planning CT scan at the left side of the image and in the nose region, which are not visible in the MV CBCT. Absolute difference images between the $3 \mathrm{CT}$ scans are calculated and shown in the bottom row of Figure 7.12. The cupping artifact in the uncorrected MV CBCT images up to 0.3 $E D$ is clearly visible at the center of the phantom. This cupping artifact is completely removed in the corrected MV CBCT images. Small differences between the KV CBCT and the MV CBCT scan are observed in the high gradient regions. Also the result of the method proposed by Kachelriess et al. is shown, but these results will be discussed in the discussion section on comparison with other methods. 


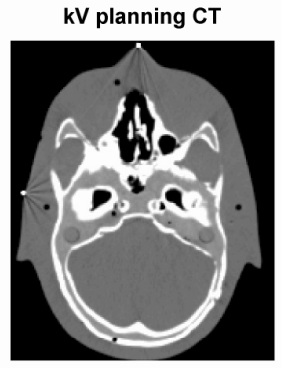

Difference corrected and uncorrected MV CBCT

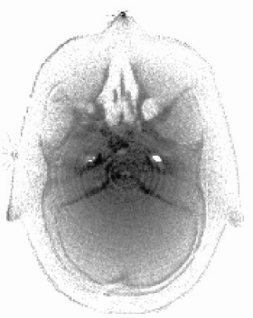

MV CBCT uncorrected

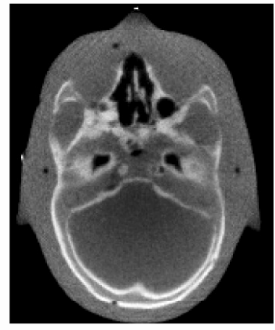

Difference kV CT and uncorrected MV CBCT

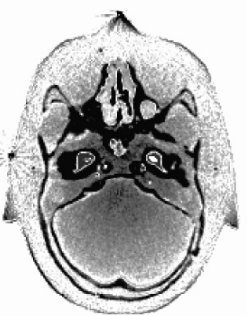

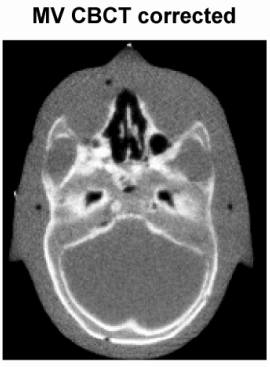

Difference $\mathrm{kV}$ CT and corrected MV CBCT

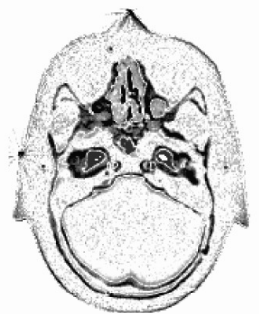

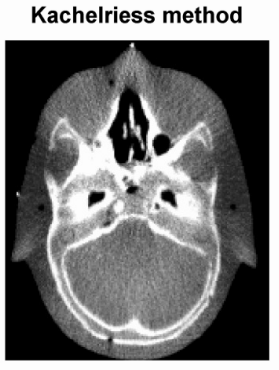

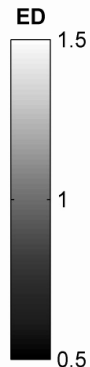

Difference $\mathrm{kV}$ CT and Kachelriess method

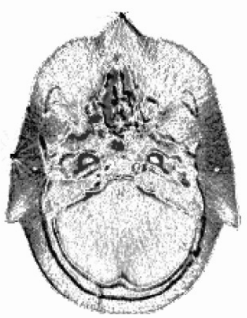

Figure 7.12: The top row shows slices of a $k V$ planning $C T$, an uncorrected and a corrected $M V$ $C B C T$ image and a MV CBCT image corrected using the Kachelriess method. In the bottom plots difference images expressed as the absolute difference in ED between the various slices are shown. The cupping artifact is removed in the corrected MV CBCT images.
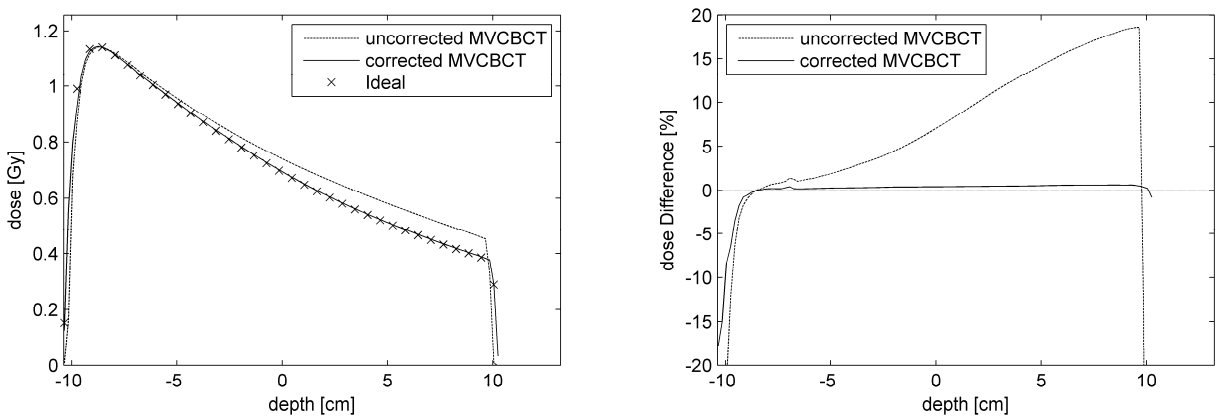

Figure 7.13: Absolute dose profiles along the central axis of the beam inside the water cylinder with a radius of $10 \mathrm{~cm}$ (left). The beam enters the cylinder at $x=-10 \mathrm{~cm}$. The ideal dose profile is calculated with the ED forced to water in the cylinder. The other profiles are obtained with the ED distributions of the corrected and uncorrected MV CBCT images. The right figure shows the relative difference in dose compared to the ideal dose distribution. 


\section{Dose calculations}

Dose calculations are performed with the ED distributions of the water cylinders with radii 5 and $10 \mathrm{~cm}$ and with the IMRT phantom with water equivalent inserts. Figure 7.13 shows the calculated dose and the relative dose differences as a function of depth along the central axis of the beam in the large cylinder. Applying the correction model results in a decrease of the maximum difference from $17.6 \%$ to $0.6 \%$ compared to the reference dose distribution. The corresponding dose difference at the center of the cylinder reduces from $7.1 \%$ to $0.4 \%$. For the IMRT phantom the maximum and mean difference reduce from $5.3 \%$ and $1.0 \%$ to $2.0 \%$ and $0.9 \%$, respectively. For the small cylinder the maximum and mean difference reduce from $2.7 \%$ and $0.3 \%$ to $0.0 \%$ and $0.2 \%$, respectively.

\section{DISCUSSION}

\section{The portal image correction model}

\section{Scatter correction}

For accurate scatter kernel fitting and scatter prediction it is important to restore the beam profile in the portal images. It is not possible to extract this profile from the gain images, because besides a correction for different segments of the EPID, the gain image inherently corrects also for the different EPID response with changing off-axis energy spectrum.

In the left plot of Figure 7.3, the effect of the scatter correction on the ED values is shown. If the scatter contribution is not subtracted from the transmission images, the estimated primary transmission is too high yielding an underestimation in the reconstructed ED of approximately $25 \%$ over the entire image. This implies that the scatter correction is necessary to get the absolute value of the ED correct, whereas the conversion from primary transmission to thickness is needed to remove the cupping artifact. This is confirmed by the reconstructions of the portal images that are not corrected for scatter but that are corrected only for beam hardening and softening and the energy dependent response of the EPID.

\section{Conversion of transmission to thickness}

Both the energy dependent response of the EPID and beam hardening and softening effects have a large influence on the primary transmission. Due to beam hardening, the energy spectrum of a rayline shifts towards higher energies as it travels through an object and the photons traveling along this rayline become relatively less attenuated. If beam hardening is ignored the radiological thickness that is extracted from the primary transmission can be up to $16 \%$ too high for a $25 \mathrm{~cm}$ thick absorber. Due to the off-axis shift in photon spectrum towards lower energies (beam softening), an off-axis rayline 
is more attenuated than an on-axis rayline. The resulting effect on the EPID signal, clearly visible in Figure 7.2, is amplified by the larger amount of attenuation of lowenergetic photons for which the EPID is highly sensitive ${ }^{27}$. If the off-axis variations in primary transmission are not taken into account, the extracted thickness can be overestimated up to $21 \%$, leading to an underestimation in electron density of $0.14 \pm$ 0.09 in the final 3D cone-beam reconstruction of the large water cylinder. The presented conversion from transmission to thickness takes into account these effects.

\section{CT number to ED conversion}

Because the Compton effect is the dominant interaction process in the Megavolt energy range, the reconstructed CT numbers increase almost linearly with electron density and a linear relation is sufficient to convert the CT numbers to ED. ${ }^{1,15,17}$ Apart from two cases, the mean ED values of all the materials of the analyzed phantoms are within 0.04 ED points from the calibration line (Figure 7.10 and Table 7.2). This confirms that a linear relation is suitable to calibrate the CT numbers to ED and that just one MV CBCT scan of a cylinder filled with water is sufficient to perform the calibration. A CT number to ED conversion for the uncorrected images is hampered mainly because the CT number depends not only on the actual ED of the insert but to a large extent also on the position and amplitude of the cupping artifact.

\section{Calculation time}

The total time needed to filter, correct and reconstruct the 200 project images was approximately 2 hours with an Intel ${ }^{\circledR}$ Core $^{\mathrm{TM}} 2 \mathrm{CPU}$ PC with $1.66 \mathrm{GHz}$ and 1 GB RAM. Filtering the images takes approximately 10 minutes, correcting the portal images 100 minutes and the cone-beam reconstruction is performed within 1 minute. No effort has been made yet to optimize the algorithm with respect to calculation time. The calculation time could be reduced by implementing the algorithm in a more low-level programming language than Matlab (Version 7.1, The MathWorks Inc., Natick, MA) we used, by applying a multi-grid approach for scatter prediction and by using more calculation power for example by multi-threading code to be compatible with the recent generation of dual and quad core CPUs. In addition it can be reduced by a factor 1.5 when a fixed number of iterations of 2 is applied. Two iterations were in all analyzed cases sufficient, but due to the specific convergence criterion that the difference between two subsequent iterations may not exceed a certain threshold, a third iteration was needed. It is estimated that the total time needed to correct the portal images can be reduced by a factor 10 or more. For the current study it was not required that the corrected scans would be instantaneously available for interpretation to use for on-line dose calculations. We think that the extraction of reliable electron density is an important step for off-line adaptive treatment, that needs to be available before the next fraction is going to be delivered. 


\section{Verification of the model}

\section{Reconstructed electron density distributions}

Applying the correction model to the portal images of the analyzed phantoms resulted in cupping free images with variations in ED below 0.03 units (1 SD), which is approximately equal to the level of noise, compared to a maximum of 0.09 if the portal images are not corrected. In three of the inserts the standard deviation (1 SD) in ED is 0.04 , but this is merely caused by the increased level of noise in the small inserts than to residual cupping. The mean ED could be reconstructed for all phantoms with an error smaller than 0.04 ED compared to the real ED, except for the bone insert in the IMRT phantom (ED = 1.51) and for the lung insert (ED = 0.32) in Phantom C. Because the $E D$ in the cork regions ( $E D=0.19$ ) in Phantom $A$ and $B$ could be reconstructed with high accuracy, it is expected that the overestimation of the ED in the lung insert is not due to its low ED. It is more probable that its small size, the large gradients in ED near the wall of the insert and its location in the center of the cupping where the ED is 0.3 units too low in the surrounding water are of importance. The CT numbers in the bone equivalent region in the IMRT phantom was $7.8 \%$ too low yielding an underestimation in ED of 0.15 units. Taken into account the underestimation in CT number of $4.6 \%$ in the entire phantom, the deviation in mean CT number in the bone insert is only $3.2 \%$ too low, which is comparable with the other inserts.

The MV CBCT images of the Rando phantom show the actual image quality that is obtained using our MV CBCT system. A clear reduction of the cupping artifact is visible in the corrected compared to the uncorrected MV CBCT images. There are some phenomena related to the use of $\mathrm{MV}$ photon beams that are disadvantageous compared to conventional $\mathrm{kV}(\mathrm{CB}) \mathrm{CT}$ scanning, such as the higher dose that is required and the reduced signal-to-noise ratio due to the different photon-interaction properties. However, the different interaction properties may also be beneficial for MV CBCT. For example, the streak artifacts resulting from high Z-material such as tooth fillings and hip prostheses are not visible in the MV CBCT images. See for example the markers at the skin of the Rando phantom. The difference images between the kV CT and the corrected and uncorrected images show some deviations in regions with large gradients in ED. This might be due to the fact that the slice of the MV CBCT was not exactly at the same position compared to the $\mathrm{kV}$ slice.

\section{Dose distributions}

The dose calculations that use the ED extracted from the uncorrected CBCT of the large cylinder reflect the effect of the cupping artifact on the dose distributions. Due to the cupping, the underestimation of the radiological thickness along the central axis of the beam causes an increased penetration depth and hence an overestimation of the dose up to $18 \%$. If the correction model is applied the maximum difference with the reference dose distributions is smaller than $1 \%$ and $2 \%$ for the water cylinders and the IMRT phantom, respectively. Dose calculations with uncorrected MV CBCT scans of objects that contain regions with ED values higher than water are likely to lead to 
larger errors than observed here, since the effect of cupping is more pronounced in high ED material than in water.

The conditions used for the present dose calculations with uncorrected MV CBCT images (i.e. large object, a single beam with a small field size) represent an extreme case. Dose calculations with smaller objects, multiple beams, larger field sizes or with beams that do not travel through the center of the cupping artifacts will result in smaller errors. However, objects with large variations in ED are expected to lead to larger deviations, since the effect of cupping becomes more pronounced as the ED increases. This means that the potential improvement of applying cupping correction procedures for dose calculations depends on the location of the tumor and the beam arrangement of the treatment plan.

\section{Comparison with other methods}

A number of methods to correct cupping artifacts have been proposed previous$1 y^{1,6,16,17,20-22,28}$ as well for MV CBCT as for kV CBCT. kV radiation has completely different scatter properties than MV radiation and in contrary to the latter it has nonuniform attenuation properties for different material compositions. This makes it more difficult to apply suitable scatter and beam hardening corrections for kv CBCT than for $\mathrm{MV}$ CBCT and hampers the subsequent conversion from CT numbers to ED. ${ }^{20}$ Therefore it is not guaranteed that methods, that were designed to correct cupping artifacts of $\mathrm{kV} C B C T$ images and to convert the images to electron density distributions, will work for MV CBCT scans. ${ }^{18-20}$

Previously, Kachelriess et al. ${ }^{22}$ designed an empirical cupping correction method for a micro kV CB-system, which passes raw projection image data through a single pixel-independent polynomial before $\mathrm{CBCT}$ reconstruction. They demonstrated that their method yields cupping free images of cylinders of different radii and of a mouse. A very attractive feature of their method is the short calculation time needed. Therefore we implemented the Kachelriess method and tested its suitability to correct MV CBCT scans of the Rando head phantom. The coefficients of the polynomial were fitted with an error smaller than $2 \%$ ( $1 \mathrm{SD}$ ) using the water equivalent cylinder with a radius of $8 \mathrm{~cm}$ and the coefficients were $-0.0063,0.643,1.109,-2.567$ and 2.161 from the zero to the fourth order, respectively. Figure 7.12 shows in the fourth column a slice of the MV CBCT scan that was corrected with the Kachelriess method and a difference image with the same slice of the KV planning CT scan. In the center of the phantom the magnitude of the differences is comparable to the current method but an increase is observed towards the boundary of the phantom up to 0.3 ED units. This might be caused by the elliptic shape of the head geometry compared to the perfect cylindrical shape of the fitting geometry, but further investigations are required to characterize the differences. Kachelriess et al. already mentioned improvements could be obtained by calculating multiple calibration coefficients for different transversal slices, this might improve the accuracy for the MV CBCT scan of the head phantom. Although the Kachelriess method can be applied to correct MV CBCT scans of objects with similar dimensions as the calibration phantom, it might be less suitable to correct the cupping artifacts in MV CBCT scans of objects with large differences in geometry compared to the calibration phantom as used in the current study. 

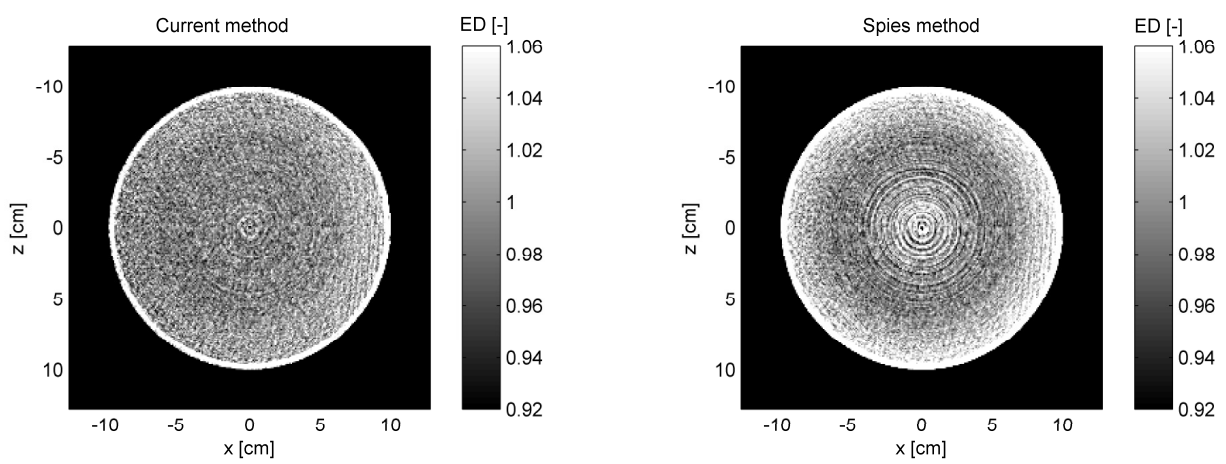

Figure 7.14: The central slice of the MV CBCT scans that are corrected with the current method $(M=3)$ (left) and with an implementation of the method by Spies et al. $(M=2)$. The right image was calibrated to ED by division by the mean CT number in the phantom. The window levels are set to the mean $E D \pm 2 S D$.

The method proposed by Spies et al. ${ }^{17}$ is similar to the presented method with respect to the use of an iterative algorithm based on transmission images to estimate the scatter. The main difference is that they derived the scatter kernels using Monte Carlo simulations, whereas we used transmission measurements based kernels. An advantage of the latter approach is that no MC expertise is needed, that the measurements are performed within an hour and that fitting the kernels is straightforward, which makes this method much easier and less time consuming to implement. Further, Spies used a CCD based EPID, whereas the a-Si EPID used in the present study is known to be much more sensitive to differences in photon energy spectrum. This might influence the performance of the cupping correction methods and limit the interchangeability of the methods for the different EPIDs. In addition, Spies et al. used a $2^{\text {nd }}$ order polynomial to map radiological thickness to primary transmission, whereas we used a third order polynomial. Although it is not expected that both methods would yield large differences in the final solutions, the method of Spies was implemented to verify this and applied to the water cylinders with radii of 5 and $10 \mathrm{~cm}$. The MV CBCT scan of the small cylinder was corrected yielding variation in ED of 0.02 (1 SD), but the mean ED was $6 \%$ higher than the mean ED of the larger cylinder. Correcting the large cylinder yielded an SD of 0.03 , but a systematic overestimation of the ED near the boundary of the cylinder (Figure 7.14). These overestimations in ED are due to discrepancies between the primary transmission data and the polynomial fit with $M=2$ for small values $(\mathrm{t}<8 \mathrm{~cm}$ ) of the radiological thickness. Spies et al. demonstrated that their method reduces cupping artifacts to $8 \%$, we have obtained a similar accuracy of $6 \%$ applying their method. However, a smaller error, at maximum $4 \%$ in terms of ED, was achieved with the current method and validated for a large range of objects with different shapes and composition.

Partridge et al. ${ }^{6}$ used a procedure that was based on the technique of Spies et al. with an a-Si EPID of $20 \times 20 \mathrm{~cm}^{2}$. The EPID size limited the field of view of the CB system at the isocenter to $15.4 \times 15.4 \mathrm{~cm}^{2}$ and therefore also the object size which has a corresponding minimal cupping artifact. No comparison was presented between the 
images with and without correction, but using the corrected images did not result in significant differences in dose compared to a kV planning CT, when both are used for an IMRT dose calculation. These authors mentioned that for full assessment of the clinical accuracy, further investigations should be carried out with a larger detector (40 $x 40 \mathrm{~cm}^{2}$ ). Also the amount of radiation (100 cGy) that was needed during acquisition was too high for clinical use.

Chen et al. ${ }^{1}$ and Morin et al. ${ }^{16}$ measured the size and shape of the cupping artifact of a homogenous cylinder and used it to correct MV CBCT images of head-and-neck patients. After correction, the cupping artifacts were smaller than $5 \%$. A limitation of their method is that the cupping was characterized for cylinders of different sizes and not necessarily valid for differences in shape and position of the cupping artifacts of other objects positioned differently.

Another issue outside the scope of this paper is the effect of the correction model on image quality, which was not considered. Although image quality is, in general, improved if cupping is removed, the impact on resolution and other imaging quality parameters needs to be determined. This is subject of further research. For dose calculations the resolution is less an issue due to the fact that the dose calculation grid of treatment planning systems usually is chosen between $2 \mathrm{~mm}$ to $5 \mathrm{~mm}$.

\section{CONCLUSION}

In this study a model is proposed capable of correcting cupping artifacts and converting CT numbers to electron density for low-dose MV CBCT images for a large range of objects with varying shapes and compositions without using prior knowledge of the geometry. An advantage of the present model compared to other approaches is that no Monte Carlo simulations are needed to describe the scatter kernels and that it can be easily implemented. If cupping artifacts are not corrected errors in dose calculations up to $17 \%$ can occur. Correcting the portal images with the present model results in cupping free images that are converted to electron density distributions and used for accurate dose calculations with errors in dose smaller than $2 \%$.

\section{ACKNOWLEDGEMENTS}

We would like to thank Siemens Oncology Care Systems for technical and financial support. The authors would also like to thank Dr. Ben Mijnheer for critically reading the manuscript and useful discussion.

\section{REFERENCES}

${ }^{1}$ J. Chen, O. Morin, M. Aubin, M.K. Bucci, C.F. Chuang, and J. Pouliot, "Dose-guided radiation therapy with megavoltage cone-beam CT," Br. J. Radiol. 79 Spec No 1, S87-98 (2006).

${ }^{2}$ V.N. Hansen, P.M. Evans, and W. Swindell, "The application of transit dosimetry to precision radiotherapy," Med. Phys. 23, 713-721 (1996). 
3.M. Kapatoes, G.H. Olivera, K.J. Ruchala, J.B. Smilowitz, P.J. Reckwerdt, and T.R. Mackie, "A feasible method for clinical delivery verification and dose reconstruction in tomotherapy," Med. Phys. 28, 528-542 (2001).

${ }^{4}$ R.J.W. Louwe, E.M.F. Damen, M. van Herk, A.W.H. Minken, O. Torzsok, and B.J. Mijnheer, "Three-dimensional dose reconstruction of breast cancer treatment using portal imaging," Med. Phys. 30, 2376-2389 (2003).

${ }^{5}$ U. Oelfke, T. Tucking, S. Nill, A. Seeber, B. Hesse, P. Huber, and C. Thilmann, "Linac-integrated kV-cone beam CT: technical features and first applications," Med. Dosim. 31, 62-70 (2006).

${ }^{6} \mathrm{M}$. Partridge, M. Ebert, and B.M. Hesse, "IMRT verification by three-dimensional dose reconstruction from portal beam measurements," Med. Phys. 29, 1847-1858 (2002).

${ }^{7}$ W.D. Renner, M. Sarfaraz, M.A. Earl, and C.X. Yu, "A dose delivery verification method for conventional and intensity-modulated radiation therapy using measured field fluence distributions," Med. Phys. 30, 2996-3005 (2003).

${ }^{8}$ W.J.C. van Elmpt, S.M.J.J.G. Nijsten, R.F.H. Schiffeleers, A.L.A.J. Dekker, B.J. Mijnheer, P. Lambin, and A.W.H. Minken, "A Monte Carlo based three-dimensional dose reconstruction method derived from portal dose images," Med. Phys. 33, 2426-2434 (2006).

${ }^{9}$ C.M. Ma and K. Paskalev, "In-room CT techniques for image-guided radiation therapy," Med. Dosim. 31, 30-39 (2006).

${ }^{10}$ S.P. Sorensen, P.E. Chow, S. Kriminski, P.M. Medin, and T.D. Solberg, "Image-guided radiotherapy using a mobile kilovoltage x-ray device," Med. Dosim. 31, 40-50 (2006).

${ }^{11}$ J.R. Wong, L. Grimm, M. Uematsu, R. Oren, C.W. Cheng, S. Merrick, and P. Schiff, "Imageguided radiotherapy for prostate cancer by CT-linear accelerator combination: prostate movements and dosimetric considerations," Int. J. Radiat. Oncol. Biol. Phys. 61, 561-569 (2005).

${ }^{12}$ B.A. Groh, J.H. Siewerdsen, D.G. Drake, J.W. Wong, and D.A. Jaffray, "A performance comparison of flat-panel imager-based MV and kV cone-beam CT," Med. Phys. 29, 967-975 (2002).

${ }^{13}$ D.A. Jaffray, J.H. Siewerdsen, J.W. Wong, and A.A. Martinez, "Flat-panel cone-beam computed tomography for image-guided radiation therapy," Int. J. Radiat. Oncol. Biol. Phys. 53, 13371349 (2002).

${ }^{14}$ L.A. Feldkamp, L.C. Davis, and J.W. Kress, "Practical cone-beam algortihm," J. Opt. Soc. Am. A 1, 612-619 (1984).

${ }^{15}$ O. Morin, A. Gillis, J. Chen, M. Aubin, M.K. Bucci, M. Roach, 3rd, and J. Pouliot, "Megavoltage cone-beam CT: system description and clinical applications," Med. Dosim. 31, 51-61 (2006).

${ }^{16}$ O. Morin, J. Chen, M. Aubin, A. Gillis, J.F. Aubry, S. Bose, H. Chen, M. Descovich, P. Xia, and J. Pouliot, "Dose calculation using megavoltage cone-beam CT," Int. J. Radiat. Oncol. Biol. Phys. 67, 1201-1210 (2007).

${ }^{17}$ L. Spies, M. Ebert, B.A. Groh, B.M. Hesse, and T. Bortfeld, "Correction of scatter in megavoltage cone-beam CT," Phys. Med. Biol. 46, 821-833 (2001).

${ }^{18}$ Y. Yang, E. Schreibmann, T. Li, C. Wang, and L. Xing, "Evaluation of on-board kV cone beam CT (CBCT)-based dose calculation," Phys. Med. Biol. 52, 685-705 (2007).

${ }^{19} \mathrm{~S}$. Yoo and F.F. Yin, "Dosimetric feasibility of cone-beam CT-based treatment planning compared to CT-based treatment planning," Int. J. Radiat. Oncol. Biol. Phys. 66, 1553-1561 (2006).

${ }^{20}$ D. Letourneau, R. Wong, D. Moseley, M.B. Sharpe, S. Ansell, M. Gospodarowicz, and D.A. Jaffray, "Online planning and delivery technique for radiotherapy of spinal metastases using cone-beam CT: image quality and system performance," Int. J. Radiat. Oncol. Biol. Phys. 67, 1229-1237 (2007).

${ }^{21}$ G. Jarry, S.A. Graham, D.J. Moseley, D.J. Jaffray, J.H. Siewerdsen, and F. Verhaegen, "Characterization of scattered radiation in kV CBCT images using Monte Carlo simulations," Med. Phys. 33, 4320-4329 (2006). 
${ }^{22}$ M. Kachelriess, K. Sourbelle, and W.A. Kalender, "Empirical cupping correction: a first-order raw data precorrection for cone-beam computed tomography," Med. Phys. 33, 1269-1274 (2006).

${ }^{23}$ J. Pouliot, A. Bani-Hashemi, J. Chen, M. Svatos, F. Ghelmansarai, M. Mitschke, M. Aubin, P. Xia, O. Morin, K. Bucci, M. Roach, 3rd, P. Hernandez, Z. Zheng, D. Hristov, and L. Verhey, "Lowdose megavoltage cone-beam CT for radiation therapy," Int. J. Radiat. Oncol. Biol. Phys. 61, 552-560 (2005).

${ }^{24}$ K.L. Pasma, B.J.M. Heijmen, M. Kroonwijk, and A.G. Visser, "Portal dose image (PDI) prediction for dosimetric treatment verification in radiotherapy. I. An algorithm for open beams," Med. Phys. 25, 830-840 (1998).

${ }^{25}$ W.J.C. van Elmpt, S.M.J.J.G. Nijsten, B.J. Mijnheer, and A.W.H. Minken, "Experimental verification of a portal dose prediction model," Med. Phys. 32, 2805-2818 (2005).

${ }^{26} \mathrm{P}$. Perona and J. Malik, "Scale-space and edge detection using anisotropic diffusion," IEEE Transactions on Pattern Analysis and Machine Intelligence 12, 629-639 (1990).

${ }^{27}$ L. Parent, J. Seco, and P.M. Evans, "Monte Carlo modelling of a-Si EPID response: The effect of spectral variations with field size and position," Med. Phys. 33, 4527-4540 (2006).

${ }^{28}$ S. Midgley, R.M. Millar, and J. Dudson, "A feasibility study for megavoltage cone beam CT using a commercial EPID," Phys. Med. Biol. 43, 155-169 (1998). 


\section{CHAPTER}

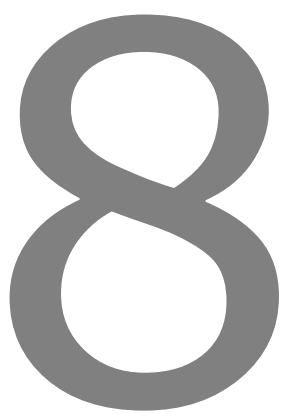

3D in vivo dosimetry using megavoltage cone-beam CT and EPID dosimetry

Wouter van Elmpt, Sebastiaan Nijsten, Steven Petit, Ben Mijnheer, Philippe Lambin and André Dekker

Published in:

International Journal of Radiation Oncology*Biology*Physics (in press), 2009. 


\begin{abstract}
Purpose: To develop a method that reconstructs, independently of previous (planning) information, the dose delivered to the patient by combining in-room imaging with transit dose measurements during treatment.

Methods and Materials: A megavoltage cone-beam CT scan of the patient anatomy is acquired with the patient in treatment position. During treatment, delivered fields are measured behind the patient with an electronic portal imaging device (EPID). The dose information in these images is back-projected through the cone-beam CT scan and used for Monte Carlo simulation of the dose distribution inside the cone-beam CT scan. Validation is performed using various phantoms for conformal and IMRT plans. Clinical applicability is shown for a head-and-neck cancer patient treated with IMRT.

Results: For single IMRT beams and a 7-field IMRT step-and-shoot plan the dose distribution was reconstructed within $3 \% / 3 \mathrm{~mm}$ compared to the measured or planned dose. A 3D conformal plan, verified using 8 point-dose measurements, resulted in a difference of $1.3 \pm 3.3 \%$ (1SD) compared to the reconstructed dose. For the patient case, planned and reconstructed dose distribution was within $3 \% / 3 \mathrm{~mm}$ for about $95 \%$ of the points within the $20 \%$ isodose line. Reconstructed mean dose values, obtained from dose-volume histograms, were within $3 \%$ of prescribed values for target volumes and normal tissues.

Conclusions: We have presented a new method that verifies the dose delivered to a patient by combining in-room imaging with the transit dose measured during treatment. This verification procedure opens possibilities for off-line adaptive radiotherapy and dose-guided radiotherapy strategies taking into account the dose distribution delivered during treatment sessions.
\end{abstract}




\section{INTRODUCTION}

Verification of the dose delivered during external beam radiotherapy is of importance to guarantee accurate delivery of treatment. There are two major sources of uncertainty that may cause the planned dose to be different from the actual dose delivered to the patient. The first possible source of discrepancy is related to the patient model (planning CT scan) in which the dose distribution is planned and calculated. This planning CT scan is usually made on a conventional CT scanner a few days or weeks before the start of treatment. Between the time of obtaining the planning CT scan and the treatment, as well as during the course of treatment, changes in patient anatomy might occur (e.g. weight loss or tumor shrinkage). A second source of uncertainty is due to errors in the modeling of the beam characteristics of the linear accelerator, limitations of the dose calculation algorithm in the treatment planning system (TPS), or to dose delivery errors of the linear accelerator during treatment (e.g. differences between planned and actual leaf positions of the multi-leaf collimator).

In the past years, various research groups investigated the use of dose reconstruction techniques to perform a verification of the dose distribution in three dimensions (3D).$^{1-7}$ These methods either verify the patient anatomy by using in-room imaging, for example using the recent developments in kilovoltage (kV) and megavoltage (MV) cone-beam CT imaging, or verify the dose delivered using another dose calculation engine (e.g. Monte Carlo). Combination of both in-room imaging and a dose calculation based on the actual delivered treatment fields will yield the ultimate verification of the dose delivery: a reconstruction of the dose delivered in the actual patient anatomy for a particular fraction, i.e. 3D in vivo dosimetry.

The aim of this study was to present and validate a model used for the 3D in vivo dose verification based on information gathered during treatment, i.e. the patient anatomy measured using MV cone-beam CT and the transit dose measured behind the patient using an Electronic Portal Imaging Device (EPID) calibrated for dosimetry purposes. $^{8}$ The method was validated using a phantom study incorporating both homogeneous and inhomogeneous phantoms for both 3D conformal and intensitymodulated fields. The clinical applicability of our model is demonstrated for a headand-neck cancer patient treated with IMRT.

\section{METHODS AND MATERIALS}

\section{Treatment delivery and portal image acquisition}

$6 \mathrm{MV}$ photon beams are delivered with a Siemens Oncor linear accelerator (Siemens Medical Solutions, Concord, CA). Portal images are acquired using an amorphous silicon-type EPID (Siemens OptiVue 1000ST, Concord, CA) with the standard acquisition software implemented in the Siemens Coherence workspace. Images have matrix sizes of $512 \times 512$ or $1024 \times 1024$ pixels with a 16-bit grey-level depth and are converted into portal dose images using an in-house developed calibration model. This model has been extensively described elsewhere. ${ }^{9}$ For the IMRT treatment fields, we applied this 
conversion model for the individual segments of a treatment beam, including a ghosting correction factor per segment. ${ }^{10}$ These converted images of the segments are then summed to a portal dose image for the entire beam.

\section{Electron density from MV CBCT}

MV cone-beam CT scans are made following the clinical protocol using a total number of 8 or 15 monitor units (MUs). The projection images of the MV cone-beam CT scan are filtered using an anisotropic diffusion filter, corrected for scattered radiation and the photon energy dependence of the response of the EPID. The projection images are then reconstructed to a $3 \mathrm{D}$ volume using a voxel-driven filtered back-projection algorithm based on the Feldkamp-algorithm. Subsequently the CT numbers are converted into electron densities using a linear conversion based on a point measurement of the CT number inside a water phantom relative to the electron density of water. This calibration procedure has been described in detail by Petit et al. ${ }^{11}$ The cone-beam CT scans are cropped to the patient contour to remove some residual noise from the images outside the patient.

\section{Back-projection of energy fluence through an MV cone-beam CT scan}

The transit portal dose images of the treatment fields are measured behind the patient and corrected for patient scattered radiation. An iterative scheme calculates the patient scattered radiation in the portal dose images using the density information of the MV cone-beam CT scan. In a first step the radiological (i.e. water equivalent) thickness $t(x, y)$ is projected from the target to every point $(x, y)$ in the detector plane. This thickness map $t(x, y)$ is defined as the sum of the electron density distribution $\rho_{e}(\vec{r})$ in the MV cone-beam CT scan along a diverging rayline $\vec{l}$ from the target to the point $(x, y)$ in the detector plane, relative to the electron density of water $\rho_{\text {water }}$ :

$$
t(x, y)=\frac{1}{\rho_{\text {water }}} \int_{0}^{(x, y)} \rho_{e}(\vec{r}) d \vec{l} .
$$

The energy fluence distribution entering the patient is calculated by correcting for the scattered radiation $S(x, y)$ produced inside the patient and the attenuation of the photon beam by the patient. The dose distribution $I_{0}(x, y)$ that would be measured in the absence of the patient is derived from the measured portal dose distribution $I(x, y)$ behind the patient by correcting for scatter and attenuation

$$
\mathrm{I}_{0}(x, y)=\frac{\mathrm{I}(\mathrm{x}, \mathrm{y})-\mathrm{s}(\mathrm{x}, \mathrm{y})}{\exp (-\mu(x, y, t(x, y)) \cdot t(x, y))},
$$

with $\mu(x, y, t)$ the effective attenuation coefficient that takes into account possible beam hardening or softening effects due the presence of the patient inside the beam, and differences in off-axis beam energy. ${ }^{12}$

The scattered dose $S(x, y)$ exiting the patient is calculated using the Equivalent Homogeneous Phantom (EHP) concept ${ }^{13}$ and pencil beam scatter kernels 


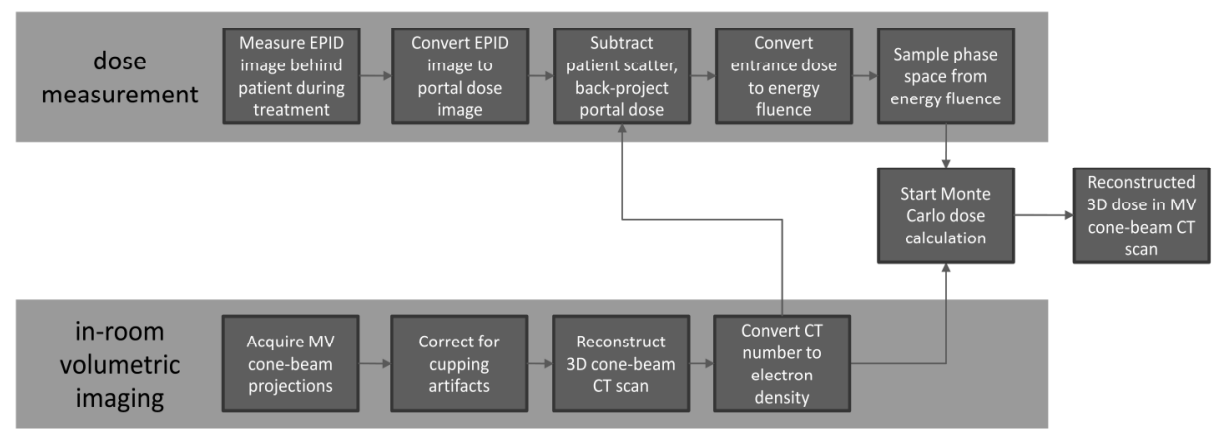

Figure 8.1: Schematic overview of the various steps in the 3D dose reconstruction method.

$$
S(x, y)=\iint I_{0}\left(x^{\prime}, y^{\prime}\right) \cdot k\left(x-x^{\prime}, y-y^{\prime}, t(x, y)\right) d x^{\prime} d y^{\prime}
$$

with $\mathrm{K}(\mathrm{x}, \mathrm{y}, \mathrm{t})$ a Gaussian-shaped pencil beam scatter kernel described in detail in a previous publication. $^{12}$

Because the calculation of the incident dose distribution $I_{0}(x, y)$ and the scattered dose distribution $S(x, y)$ depend on each other, an iterative loop is introduced. The steps above [i.e. Eq. (2-3)] are repeated 3 times, which is necessary to assure convergence of the iterative scheme. ${ }^{11,14}$ The scattered dose distribution is set to zero for the first loop.

The dose distribution $I_{0}(x, y)$ is then converted into an energy fluence distribution $\Psi_{0}(\mathrm{x}, \mathrm{y})$

$$
\Psi_{0}(x, y)=\frac{I_{0}(x, y) \otimes^{-1} K_{\Psi}(x, y)}{\mu(\theta) / \rho \exp \left(-\mu(\theta) \cdot t_{w}\right)},
$$

by correcting for the phantom scatter inside the (artificial) layer of water $\left(t_{w}=5.0 \mathrm{~cm}\right)$ using the energy deposition kernel $K_{\Psi}(x, y)$. Values for this kernel were taken from van Elmpt et al. ${ }^{15}$ This step is necessary because our EPID calibration model converts an EPID image into a dose distribution at a depth $t_{w}=5 \mathrm{~cm}$ inside a water phantom. The attenuation coefficient $\mu(\theta)$ was set to $4.68 \cdot 10^{-2} \mathrm{~cm}^{-1}$ with the off-axis correction proposed by Tailor et al. ${ }^{16}$

From the incident energy fluence distribution $\Psi_{0}(x, y)$ a phase-space is sampled assuming that all photons originate from a point source at the target of the linac. A 3D dose calculation that takes inhomogeneities into account is started from this phasespace using the fast Monte Carlo code $\mathrm{XVMC}^{17}$ using the electron density information of the MV cone-beam CT scan. Previously, the accuracy of this dose reconstruction method for pre-treatment verification (i.e. based on EPID images without an object and the planning CT scan) was assessed to be within $3 \%$ for homogeneous ${ }^{15}$ and inhomogeneous phantoms ${ }^{18}$, whereas its clinical applicability was also shown ${ }^{3}$. A schematic overview of the various steps involved in the 3D dose reconstruction procedure is shown in Figure 8.1. 

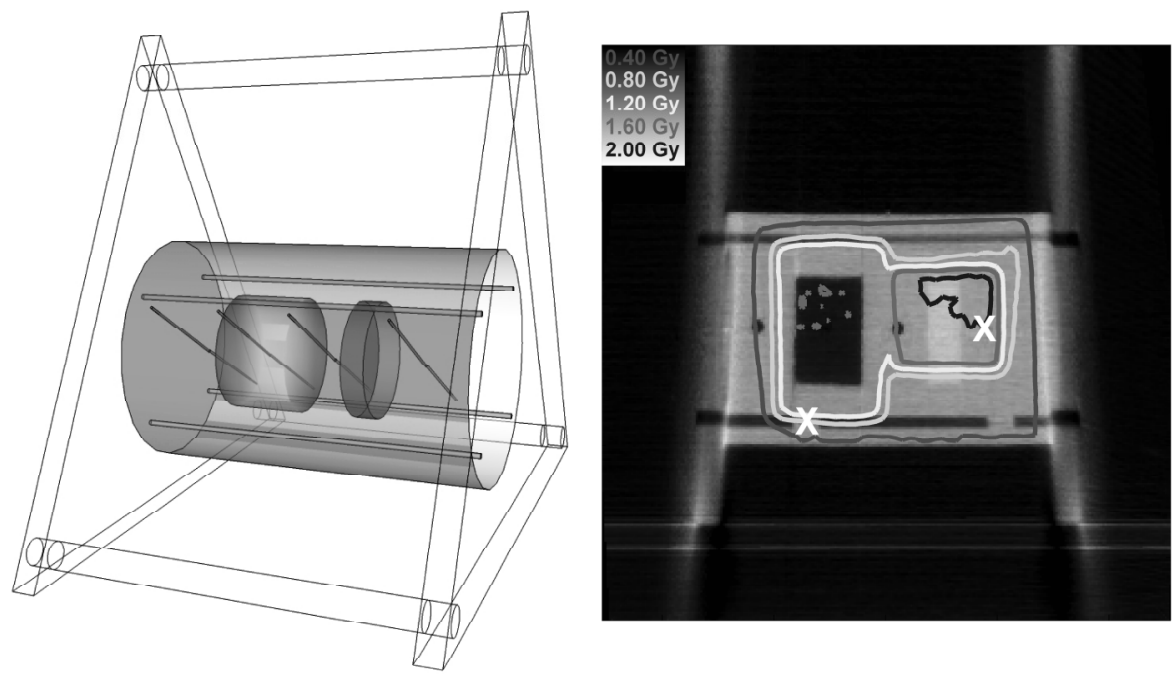

Figure 8.2: Schematic picture of the cylindrical phantom (phantom 2) having a low-density and high-density region (left) and a slice through the MV cone-beam CT scan with the reconstructed dose distribution (right). The white crosses indicate the position of a MOSFET detector.

\section{Phantom verification measurements}

The method was tested using cubic and cylindrical shaped phantoms irradiated using a $6 \mathrm{MV}$ photon beam with 3D conformal as well as intensity-modulated fields. These experiments are described in detail in the following sections.

Because the field-of-view (FOV) of the MV cone-beam CT scan is currently limited to a volume of approximately $27 \times 27 \times 27 \mathrm{~cm}^{3}$, we choose phantoms with similar dimensions as applied for the verification of head-and-neck treatments. Other treatment sites require either a larger FOV of the MV cone-beam CT or a combined registration of the planning CT scan with the MV CBCT scan to compensate for the missing anatomy outside the FOV and are subject of further study and beyond the scope of this paper. ${ }^{19}$

\section{Single IMRT field verification}

A cubic phantom made of polystyrene with dimensions $29.0 \times 14.5 \times 12.8 \mathrm{~cm}^{3}$ (phantom 1) positioned symmetrically around the isocenter was irradiated with a 6 MV IMRT field consisting of 5 segments each delivered with 40 MUs having a field size of $20 \times 10$ $\mathrm{cm}^{2}$ reduced to $4 \times 10 \mathrm{~cm}^{2}$ by closing the leaves in four steps of $4 \mathrm{~cm}$. An MV cone-beam $\mathrm{CT}$ scan having $8 \mathrm{MUs}$ was used for imaging the phantom geometry. Dose verification was performed using film measurements (EDR2, Eastman Kodak Company, Rochester, NY) calibrated for relative dose measurements. Planned 2D dose distributions in a plane inside the phantom at $4.2 \mathrm{~cm}$ and $8.6 \mathrm{~cm}$ depth were compared with the corres- 
ponding 3D reconstructed dose distributions. The film measurements were normalized to the reconstructed dose value at a point in the center of the second segment. Differences are quantified using the gamma evaluation ${ }^{20}$ using a $3 \%$ of the maximum dose and $3 \mathrm{~mm}$ distance-to-agreement criterion within the $5 \%$ isodose line.

\section{D conformal technique verification}

To verify the dose reconstruction also in inhomogeneous cases, a cylindrical phantom of $11 \mathrm{~cm}$ diameter and $14 \mathrm{~cm}$ length was made of flexible silicon material $\left(\rho \approx 1.2 \mathrm{~g} / \mathrm{cm}^{3}\right)$ which included a low-density foam region $\left(\rho \approx 0.1 \mathrm{~g} / \mathrm{cm}^{3}\right)$ of $5 \mathrm{~cm}$ diameter and $3 \mathrm{~cm}$ length (phantom 2), as well as a higher density PMMA disk $\left(\rho \approx 1.4 \mathrm{~g} / \mathrm{cm}^{3}\right)$ of $5 \mathrm{~cm}$ diameter and $1.5 \mathrm{~cm}$ length. A schematic picture of the phantom is shown in Figure 8.2. The phantom was irradiated with a $6 \mathrm{MV}$ 3-beam conformal technique, with one $50 \mathrm{MU}$ field of $9.8 \times 13.0 \mathrm{~cm}^{2}$ at 0-degree gantry angle and a virtual wedge of $45 \mathrm{de}-$ grees; the second beam was a small field of $3.8 \times 5.0 \mathrm{~cm}^{2}$ with $150 \mathrm{MUs}$ at 60 -degree gantry angle and the third field had a size of $7.6 \times 5.0 \mathrm{~cm}^{2}$ with $100 \mathrm{MUs}$ at 270 degrees. To validate the 3D dose reconstruction method, calibrated MOSFET detectors (TN502RD, Thomson and Nielsen Electronics, Ottawa, ON, Canada) were positioned inside this phantom at 8 different points located throughout the entire volume. The MOSFET measurements were repeated 3 times to reduce the measurement error in MOSFET readings. $^{21}$

\section{Comparison with the TPS}

Verification of the 3D reconstructed dose distribution was performed for a clinical IMRT plan. A $20 \mathrm{~cm}$ diameter cylindrical phantom having a polystyrene wall of $5 \mathrm{~mm}$ thickness and filled with water was irradiated using a clinical IMRT plan designed for a head-and-neck cancer patient. Seven fields were delivered consisting of in total 92 segments and 528 MUs. The reconstructed dose distribution is compared to the 3D dose distribution obtained from the clinically applied treatment planning system (XiO 4.3.4, CMS, St. Louis, MO). A 2D gamma analysis within the $20 \%$ isodose line is performed in the transversal, coronal and sagittal slice through the isocenter using a $3 \%$ of the maximum dose and a $3 \mathrm{~mm}$ distance-to-agreement criterion.

\section{Clinical case study}

As an example to illustrate how our 3D dose reconstruction method is applied in clinical practice, we used the treatment of a nasopharyngeal cancer patient with an 8field step-and-shoot IMRT technique having 484 MUs delivered in 97 segments resulting in $56 \mathrm{~Gy}$ in 28 fractions. To verify the 3D dose distribution delivered to this patient, an MV cone-beam CT scan using 8 MUs was made during the $7^{\text {th }}$ fraction and the treatment fields were captured using the EPID. A 3D dose reconstruction inside the cone-beam CT scan of this patient was performed. One of the beams included a couch rotation. Because of a possible collision between the gantry and the EPID it was not possible to capture this treatment field, hence a portal dose image without a patient in the beam was used in the calculation of the energy fluence in Eq. (4). The recon- 
structed dose distribution is compared with the planned dose distribution inside the $\mathrm{kV}$ planning CT scan using 2D gamma analysis applying a $3 \%$ of the maximum dose and $3 \mathrm{~mm}$ distance-to-agreement criterion evaluated within the $20 \%$ isodose line. Because the dose reconstruction is performed early during treatment, no large anatomical differences are expected and dose-volume histograms are calculated using structures delineated in the planning CT scan.

\section{RESULTS}

\section{Phantom study}

\section{Single IMRT field verification}

Two planes extracted from the 3D dose distribution inside the MV cone-beam CT scan have been compared to the measured film dose distributions. A single plane extracted from the dose distribution at $8.6 \mathrm{~cm}$ depth is shown in Figure 8.3 together with the measured film dose distribution. A gamma evaluation for values larger than $5 \%$ of the maximum dose was used to compare both dose distributions. Good agreement was observed as shown by median gamma values of 0.149 and 0.141 for the film positioned at 4.2 and $8.6 \mathrm{~cm}$, respectively. The maximum gamma value of a single pixel for both planes was 0.89 and 0.80 , respectively.

\section{D conformal technique verification}

The 8 dose values obtained with the MOSFETs have been compared to the dose values reconstructed at the position of these detectors for phantom 2 . The average difference between the 8 MOSFET measurements and 3D reconstructed values was small, on average $1.3 \pm 3.3 \%$ ( $1 \mathrm{SD}$ ) expressed relative to the maximum dose of $2 \mathrm{~Gy}$. The largest observed deviation was $6.4 \%$ for a superficially placed MOSFET adjacent to the high-
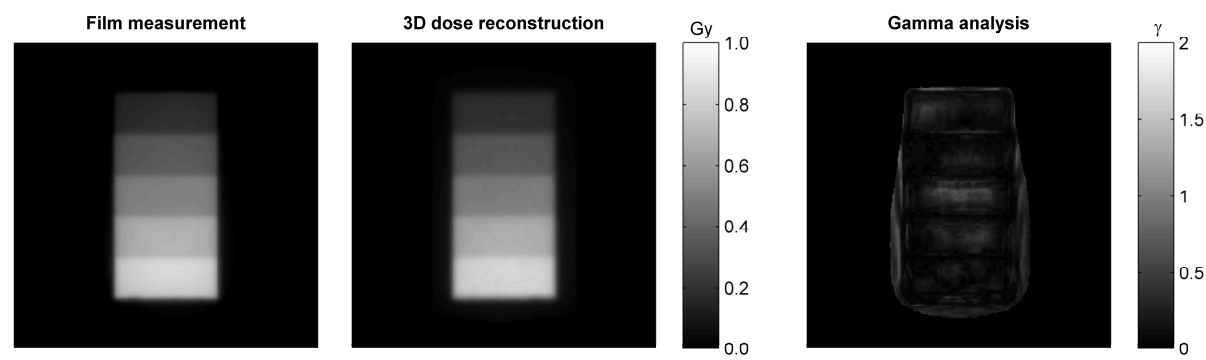

Figure 8.3: Dose distribution in a plane at $6.8 \mathrm{~cm}$ depth inside the cubic phantom (phantom 1). Film measurement (left) and reconstructed dose distribution (middle). The gamma evaluation (3\% max. dose $/ 3 \mathrm{~mm}$ ) is shown at the right. 
density region. A slice of the MV cone-beam CT scan through the isocenter with the dose distribution is shown in Figure 8.2 together with the position of two MOSFET detectors.

\section{IMRT verification}

To verify the accuracy of the 3D dose reconstruction method for an IMRT treatment, the $3 \mathrm{D}$ reconstructed dose distribution was compared to the TPS planned dose distribution for a homogenous water-filled cylinder irradiated with the 7-field IMRT technique. Both dose distributions were in good agreement with each other, indicated by the low values of the 2D gamma analysis in the various planes. The mean gamma value within the $20 \%$ isodose line was $0.253,0.293$ and 0.359 for the transversal, coronal and sagittal plane, respectively. The percentage of points with a gamma value smaller than unity for these planes was $100 \%, 99.8 \%, 100 \%$, respectively. Also scored were the gamma values of the upper percentile, which were $0.74,0.79$ and 0.84 , for the transversal, coronal and sagittal plane, respectively. Figure 8.4 shows the dose distribution of the TPS calculation overlaid with the 3D dose reconstruction method for the 3 orientations through the isocenter.

\section{Clinical example}

Figure 8.5 shows the planning CT scan with the planned dose distribution and the MV cone-beam CT scan with the 3D reconstructed dose. From an imaging point of view, no large anatomical differences can be observed between the planning CT scan and the on-line cone-beam CT scan. In order to quantify possible dosimetric differences, the dose distribution has been compared using a gamma evaluation. Good agreement is obtained between the planned and measured dose distribution indicated by low gamma values. For the transversal and sagittal slice shown in Figure 8.5, 93.5\% and $95.2 \%$ of the points, respectively, had a gamma value $<1$ with a median gamma value of 0.364 and 0.292 , respectively. The gamma evaluation reveals some small dose differences in the regions around the air cavities.

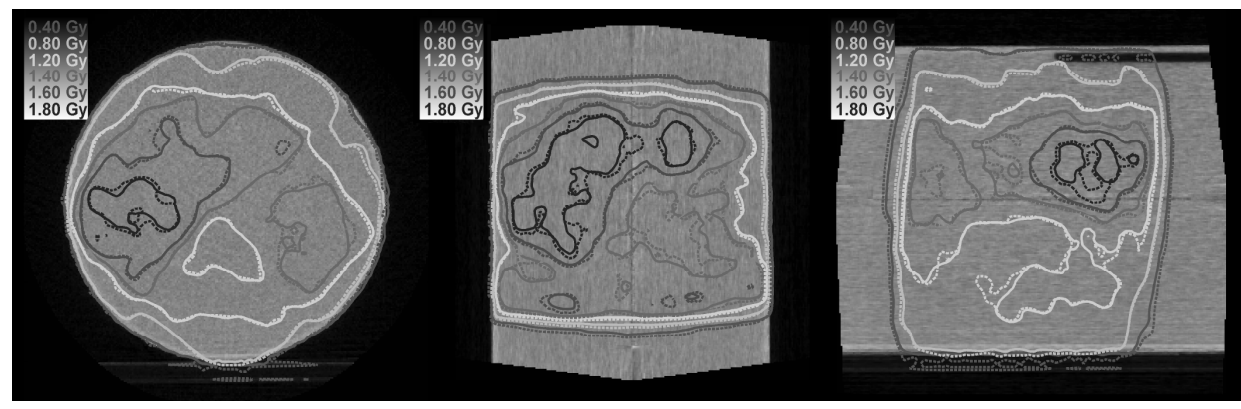

Figure 8.4: Transversal, coronal and sagittal slice of the MV cone-beam CT scan of a $20 \mathrm{~cm}$ diameter water-filled cylinder with the dose distribution calculated by the TPS (solid lines) and the $3 D$ reconstructed dose distribution (dashed lines). 
Figure 8.6 shows the dose-volume histograms (DVHs) of the planned and reconstructed dose distributions. The DVHs of the reconstructed dose of the fraction that was verified are scaled to the situation for 28 fractions to make a comparison with the planned DVH. For the PTV, a small under-dosage $(0.9 \%)$ was observed in mean dose between planned (56.4 Gy) and reconstructed dose (55.9 Gy) for the high-risk PTV. The mean dose in the low-risk PTV was $1.7 \%$ lower, $51.8 \mathrm{~Gy}$ vs. $50.9 \mathrm{~Gy}$ for the planned and reconstructed dose distribution, respectively. For the normal structures, also no large differences were observed. For instance, the maximum dose in the spinal cord was $25.7 \mathrm{~Gy}$ vs. $25.9 \mathrm{~Gy}$, the mean dose in the brain $9.0 \mathrm{~Gy}$ vs. $9.2 \mathrm{~Gy}$, the mean dose in the left eye bulb 35.9 Gy vs. $34.3 \mathrm{~Gy}$ and for the right eye $23.2 \mathrm{~Gy}$ vs. $23.5 \mathrm{~Gy}$ for the planned vs. reconstructed dose, respectively.

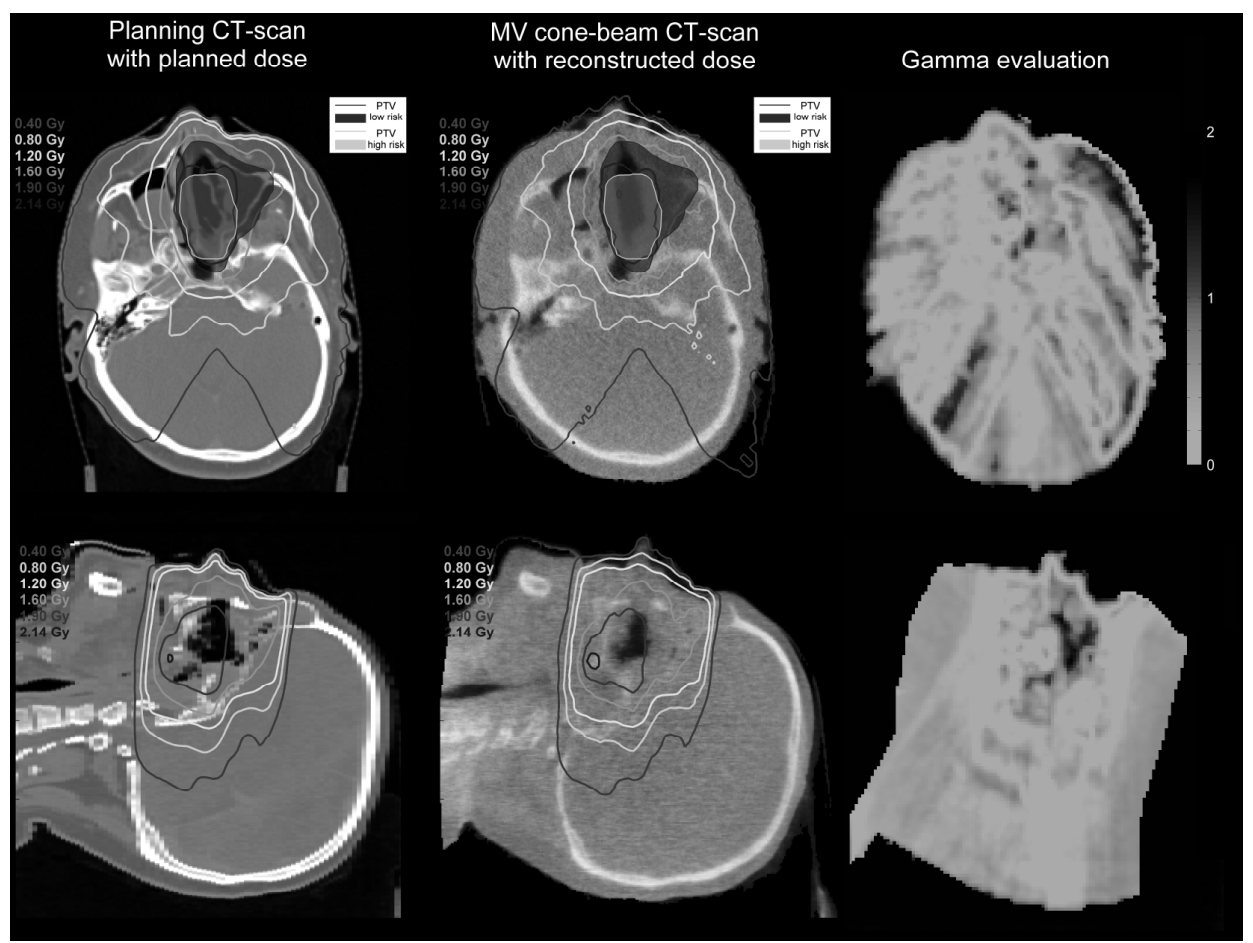

Figure 8.5: Transversal (top row) and sagittal (bottom row) slices through the planning CT scan (left) with the dose calculated by the TPS, the MV cone-beam CT scan with the reconstructed dose distribution (middle) and the gamma evaluation (right) of the comparison between the planned and reconstructed dose distribution. Small differences are visible at the edges of the air cavities. 


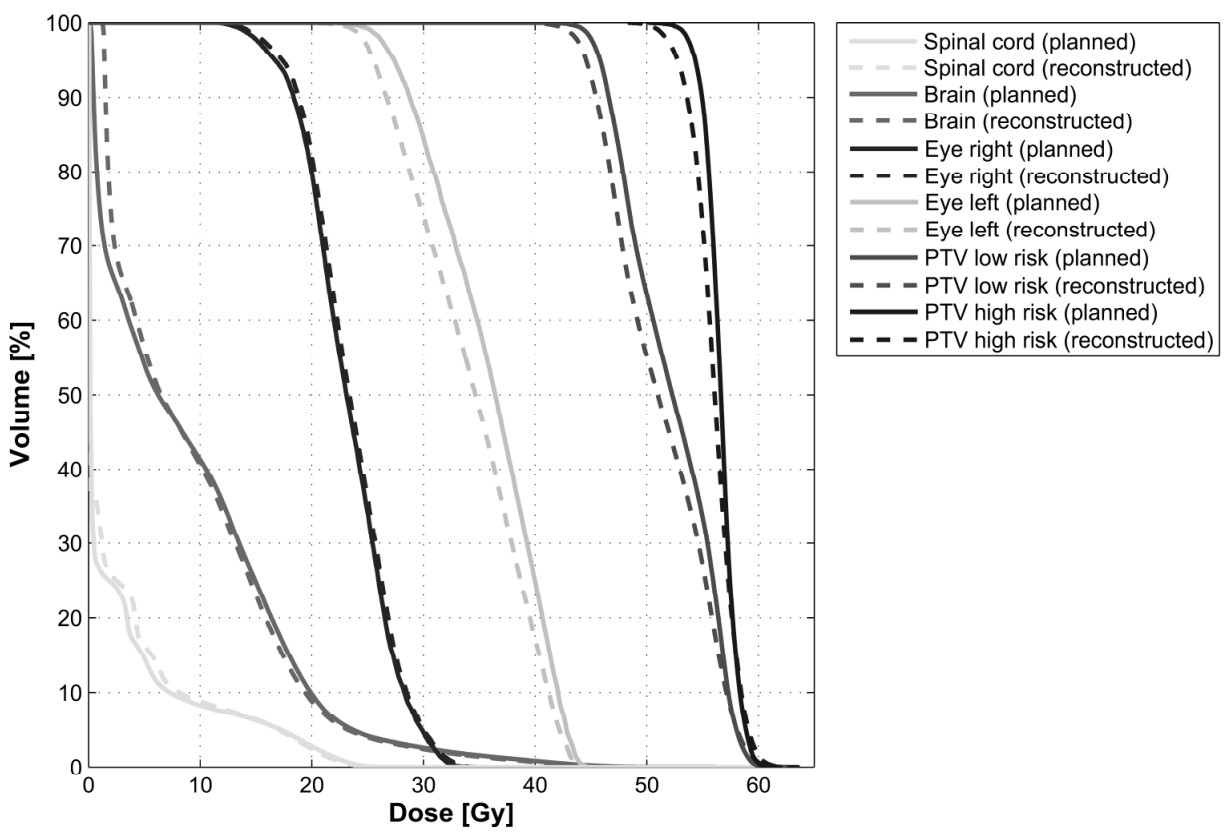

Figure 8.6: Dose-volume histograms of the planned and reconstructed dose distribution of the low- and high-risk PTV and various normal structures.

\section{DISCUSSION}

In this study, we present a new procedure to reconstruct the 3D dose distribution delivered to the patient that is completely independent of the treatment planning process, both with respect to patient anatomy and the dosimetric aspects. For the actual patient anatomy in treatment position, an MV cone-beam CT scan, calibrated for dose calculation, is used. For the fields delivered during treatment, the transit portal dose images behind the patient are acquired as a measure of the dose delivered during treatment. Differences between planned and delivered dose distributions are incorporated in this step, while in addition possible errors or inaccuracies in the dose calculation of the treatment planning system are taken into account by using an independent dose calculation engine based on Monte Carlo simulations.

The advantage and novelty of this $3 \mathrm{D}$ dose reconstruction method compared to other dose recalculation methods ${ }^{7,19,22,23}$ is that the actual delivered beams are used to reconstruct the dose delivered to the patient. If the MV cone-beam CT scan information is already used for adjusting the patient set-up then, no extra dose has to be delivered to the patient. Strategies for taking the imaging dose of the MV cone-beam CT scan into account during IMRT optimization have been described by Morin et al. ${ }^{24}$ The method does not require additional measurements, patient treatment time or 
equipment and can be performed with a modern linear accelerator equipped with an EPID and (MV) cone-beam CT technology. Other imaging modalities, including in-room conventional CT or kV cone-beam CT technology, can also be used for our dose reconstruction method provided that CT images are calibrated into electron density values. ${ }^{23}$

The time needed to reconstruct the 3D dose for the head-and-neck case was approximately 2 hours for an 8-field IMRT step-and-shoot treatment. Conversion of the portal images to portal dose images takes less than 10 minutes, the correction of the cupping artifact in the MV cone-beam CT images takes approximately 1 hour on a 2.4 $\mathrm{GHz}$ Intel Core 2 Duo processor and the dose calculation using the Monte Carlo XVMC code takes less than 20 minutes on the same machine for a statistical accuracy better than $2 \%$. This procedure is fast enough for off-line verification of a fractionated treatment where the $3 \mathrm{D}$ reconstructed dose information has to be available before the next fraction, typically the next day.

The 3D dose reconstruction method consists of various steps that all have their own uncertainty. The EPID calibration method calculates portal dose values with an uncertainty of less than $3 \%(1 \mathrm{SD}),{ }^{9}$ the calibration of the MV cone-beam CT scan into electron density values results in maximum differences of $2 \%$ in the dose distribution ${ }^{11}$ and the 3D Monte Carlo based dose calculation evaluated for head-and-neck cancer patients showed differences smaller than $2 \%(1 \mathrm{SD})$ in the target volume compared to the TPS $^{3}$. Combining these individual uncertainties would result in an upper limit of the order of $4 \%$ ( 1 SD) for the total uncertainty in our dose reconstruction method. This indicates that the method is suitable for detecting dose differences of 5 to $7 \%$ (1 SD).

A cone-beam CT scan is typically made prior to treatment while the measured portal images detect intra-fraction motion of organs during treatment. Our method reconstructs the delivered energy fluence in this patient model acquired prior to treatment. To fully take into account intra-fraction motion in the 3D dose reconstruction model, volumetric imaging during irradiation must be performed. However, such 3D imaging techniques during treatment are currently not available.

For the clinical case, small differences can be observed between the 3D dose distribution calculated by the TPS using the planning CT scan, and the 3D reconstructed dose distribution using the MV cone-beam CT data. The reconstructed dose values using the Monte Carlo simulation are in good agreement with the dose values calculated using the superposition algorithm of the TPS, although overall a slightly lower (about 1-2\%) dose is observed. Considering the fact that many deviations may occur during treatment compared to the planning, e.g. anatomical changes, variation in output of the linear accelerator, small differences in MLC leaf position, these small differences indicate that a good overall quality is achieved for the entire treatment delivery during this treatment fraction. Small differences, predominantly around the air cavities, occur in low-density regions where non-equilibrium of the scattered photons and electrons exists. Around the air cavities, the position of the reconstructed isodose lines differs somewhat from the position of the isodose lines calculated by the TPS which are more collapsed inside the patient. This is probably due to difficulties in accurately predicting energy deposition by the TPS for such configurations.

In clinical practice, it is sometimes difficult to directly compare the reconstructed dose distribution at a slice of an in-room cone-beam (or repeated conventional) CT scan with the original dose distribution calculated in a planning CT scan. Because 
changes in both anatomy and dose distribution may occur, it may not always be sufficient to apply simple rigid registration models for a comparison on a voxel-to-voxel basis. A simple but cumbersome solution would be to delineate the structures of interest in the in-room CT scan and calculate the DVH parameters of interest. It should be realized however, that delineation of structures of interest may be difficult because the image quality of in-room imaging modalities is generally less good than that of a CT scanner used for planning and contrast enhancement, often in combination with PET and MR information. Another solution that might be of interest is the use of non-rigid deformation models. However, this approach has its limitations. Voxels may disappear or appear because changes in tumor or normal tissue volume may occur during the course of treatment. This is an active area of research and needs further investigation with respect to our specific application. ${ }^{25}$ With this final step, a cumulative measurement of the actual dose delivered to the patient in the target volume and normal tissue is possible which may benefit off-line adaptive radiation therapy (ART) and eventually true dose-guided radiotherapy (DGRT).

\section{CONCLUSIONS}

In this work we have described a new model that is able to perform 3D in vivo dosimetry based on in-room MV cone-beam CT imaging and a dose measurement during treatment using an EPID. The accuracy was assessed using phantom measurements and a comparison was made with the dose calculations performed by the treatment planning system. These verification measurements indicate that the error was smaller than $3 \%$ or $3 \mathrm{~mm}$ for most of the points in the 3D reconstructed volume for both conformal and IMRT treatments. The clinical applicability of our model for 3D in vivo dosimetry was demonstrated for a head-and-neck cancer patient treatment with IMRT.

\section{ACKNOWLEDGEMENTS}

The authors would like to thank Stein Fekkes, MSc for building the heterogeneous phantom.

\section{REFERENCES}

${ }^{1}$ L. Lee, Q.T. Le, and L. Xing, "Retrospective IMRT Dose Reconstruction Based on Cone-Beam CT and MLC Log-File," Int J Radiat Oncol Biol Phys 70, 634-644 (2008).

${ }^{2}$ L.N. McDermott, M. Wendling, J. Nijkamp, A. Mans, J.J. Sonke, B.J. Mijnheer, and M. van Herk, "3D in vivo dose verification of entire hypo-fractionated IMRT treatments using an EPID and cone-beam CT," Radiother Oncol 86, 35-42 (2008).

${ }^{3}$ W. van Elmpt, S. Nijsten, B. Mijnheer, A. Dekker, and P. Lambin, "The next step in patientspecific QA: 3D dose verification of conformal and intensity-modulated RT based on EPID dosimetry and Monte Carlo dose calculations," Radiother Oncol 86, 86-92 (2008).

${ }^{4} J$. Chen, O. Morin, M. Aubin, M.K. Bucci, C.F. Chuang, and J. Pouliot, "Dose-guided radiation therapy with megavoltage cone-beam CT," Br J Radiol 79 Spec No 1, S87-98 (2006). 
${ }^{5}$ M. van Zijtveld, M.L. Dirkx, H.C. de Boer, and B.J. Heijmen, "3D dose reconstruction for clinical evaluation of IMRT pretreatment verification with an EPID," Radiother Oncol 82, 201-207 (2007).

${ }^{6}$ R.J. Louwe, E.M. Damen, M. van Herk, A.W. Minken, O. Torzsok, and B.J. Mijnheer, "Threedimensional dose reconstruction of breast cancer treatment using portal imaging," Med Phys 30, 2376-2389 (2003).

${ }^{7}$ M. van Zijtveld, M. Dirkx, and B. Heijmen, "Correction of conebeam CT values using a planning CT for derivation of the "dose of the day"," Radiother Oncol 85, 195-200 (2007).

${ }^{8}$ W. van Elmpt, L. McDermott, S. Nijsten, M. Wendling, P. Lambin, and B. Mijnheer, "A literature review of electronic portal imaging for radiotherapy dosimetry," Radiother Oncol 88, 289309 (2008).

${ }^{9}$ S.M. Nijsten, W.J. van Elmpt, M. Jacobs, B.J. Mijnheer, A.L. Dekker, P. Lambin, and A.W. Minken, "A global calibration model for a-Si EPIDs used for transit dosimetry," Med Phys 34, 38723884 (2007).

${ }^{10}$ L.N. McDermott, S.M. Nijsten, J.J. Sonke, M. Partridge, M. van Herk, and B.J. Mijnheer, "Comparison of ghosting effects for three commercial a-Si EPIDs," Med Phys 33, 2448-2451 (2006).

${ }^{11}$ S.F. Petit, W.J. van Elmpt, S.M. Nijsten, P. Lambin, and A.L. Dekker, "Calibration of megavoltage cone-beam CT for radiotherapy dose calculations: correction of cupping artifacts and conversion of CT numbers to electron density," Med Phys 35, 849-865 (2008).

${ }^{12}$ W.J. van Elmpt, S.M. Nijsten, B.J. Mijnheer, and A.W. Minken, "Experimental verification of a portal dose prediction model," Med Phys 32, 2805-2818 (2005).

${ }^{13}$ K.L. Pasma, B.J. Heijmen, M. Kroonwijk, and A.G. Visser, "Portal dose image (PDI) prediction for dosimetric treatment verification in radiotherapy. I. An algorithm for open beams," Med Phys 25, 830-840 (1998).

${ }^{14}$ L. Spies, M. Partridge, B.A. Groh, and T. Bortfeld, "An iterative algorithm for reconstructing incident beam distributions from transmission measurements using electronic portal imaging," Phys Med Biol 46, N203-211 (2001).

${ }^{15}$ W.J. van Elmpt, S.M. Nijsten, R.F. Schiffeleers, A.L. Dekker, B.J. Mijnheer, P. Lambin, and A.W. Minken, "A Monte Carlo based three-dimensional dose reconstruction method derived from portal dose images," Med Phys 33, 2426-2434 (2006).

${ }^{16}$ R.C. Tailor, V.M. Tello, C.B. Schroy, M. Vossler, and W.F. Hanson, "A generic off-axis energy correction for linac photon beam dosimetry," Med Phys 25, 662-667 (1998).

${ }^{17} \mathrm{M}$. Fippel, "Fast Monte Carlo dose calculation for photon beams based on the VMC electron algorithm," Med Phys 26, 1466-1475 (1999).

${ }^{18}$ W.J. van Elmpt, S.M. Nijsten, A.L. Dekker, B.J. Mijnheer, and P. Lambin, "Treatment verification in the presence of inhomogeneities using EPID-based three-dimensional dose reconstruction," Med Phys 34, 2816-2826 (2007).

${ }^{19}$ J.F. Aubry, J. Pouliot, and L. Beaulieu, "Correction of megavoltage cone-beam CT images for dose calculation in the head and neck region," Med Phys 35, 900-907 (2008).

${ }^{20}$ D.A. Low, W.B. Harms, S. Mutic, and J.A. Purdy, "A technique for the quantitative evaluation of dose distributions," Med Phys 25, 656-661 (1998).

${ }^{21} \mathrm{P}$. Scalchi and P. Francescon, "Calibration of a mosfet detection system for 6-MV in vivo dosimetry," Int J Radiat Oncol Biol Phys 40, 987-993 (1998).

${ }^{22}$ O. Morin, J. Chen, M. Aubin, A. Gillis, J.F. Aubry, S. Bose, H. Chen, M. Descovich, P. Xia, and J. Pouliot, "Dose calculation using megavoltage cone-beam CT," Int J Radiat Oncol Biol Phys 67, 1201-1210 (2007).

${ }^{23}$ S. Yoo and F.F. Yin, "Dosimetric feasibility of cone-beam CT-based treatment planning compared to CT-based treatment planning," Int J Radiat Oncol Biol Phys 66, 1553-1561 (2006). 
${ }^{24}$ O. Morin, A. Gillis, M. Descovich, J. Chen, M. Aubin, J.F. Aubry, H. Chen, A.R. Gottschalk, P. Xia, and J. Pouliot, "Patient dose considerations for routine megavoltage cone-beam CT imaging," Med Phys 34, 1819-1827 (2007).

${ }^{25}$ J. Orban de Xivry, G. Janssens, G. Bosmans, M. De Craene, A. Dekker, J. Buijsen, A. van Baardwijk, D. De Ruysscher, B. Macq, and P. Lambin, "Tumour delineation and cumulative dose computation in radiotherapy based on deformable registration of respiratory correlated CT images of lung cancer patients," Radiother Oncol 85, 232-238 (2007). 

DISCUSSION 



\section{CHAPTER}

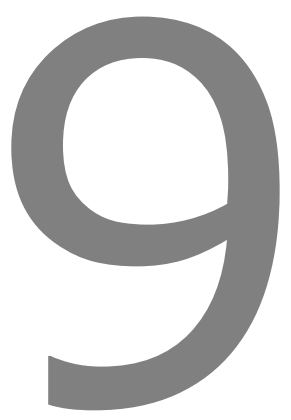

General discussion and future perspectives 



\section{GENERAL DISCUSSION}

Radiotherapy technologists, oncologists and physicists are nowadays familiar with 3D imaging data sets and 3D dose distributions. 3D CT scans, and even 4D CT scans to take respiratory motion into account, are used for state-of-the-art treatment planning. Sometimes multi-modality images are created by adding PET or MRI information. Treatment plans are designed in 3D using a treatment planning system (TPS) and aim for coverage of the target volume in all directions.

\section{Evolution from point dose and 2D verification to 3D dose verification}

In the past, verification procedures frequently used point detectors ${ }^{1,2}$ or $2 \mathrm{D}$ measurements in a plane in front of ${ }^{3-5}$, inside or behind $^{6-10-15}$ a phantom or patient. Chapter 2 gives a comprehensive overview of the various methods and models applying EPIDs mainly for point and 2D dose verification. Although 2D dose verification using EPIDs is currently clinically applied in our institute as the standard tool for large-scale routine dose verification ${ }^{16}$, these $2 \mathrm{D}$ images are not always easy to interpret in terms of tumor dose or dose in the normal tissue. It is therefore expected that verification of the dose in $3 \mathrm{D}$ will result in a more intuitive interpretation of possible dose differences compared to a verification using a $2 \mathrm{D}$ dose plane inside a phantom or a measurement of the dose or energy fluence at the EPID level. The models and methods described in this thesis for dose verification take into account the third dimension. The introduction of this $3 \mathrm{D}$ verification in clinical routine in the upcoming years will bring back the verification and interpretation of dose distributions to the same level used in treatment planning, where most professionals (including physicists and physicians) are familiar with. The use of dose-volume histograms (DVHs) and techniques such as overlapping isodose surfaces or a gamma evaluation can facilitate a comparison and interpretation of the planned dose distribution with the reconstructed dose distribution delivered to the patient. Hence, 3D dose verification will be preferred from the point of ease of interpretation.

\section{Dose calculation accuracy}

Measurements of the dose distribution in 3D inside the patient are not directly possible and dose calculation algorithms or dose reconstruction models are necessary to estimate this 3D dose distribution. The dose calculation accuracy is mainly determined by two factors. First, accurate modeling of treatment machine specific parameters is necessary. Parameters such as lateral dose profiles, depth doses, transmission through the jaws, modeling of the multi-leaf collimator $(\mathrm{MLC})$, are the starting point of a dose calculation algorithm. The second factor that determines the accuracy of the calculated dose distributions is the energy deposition algorithm implemented in the treatment planning system. For this purpose it is necessary to know, for instance, to what extent corrections for beam hardening are taken into account, corrections for tissue inhomogeneities are modeled, and lateral scattering of photons and electrons for the different tissue types is handled. 
In this thesis, both of the above mentioned factors are determined independently from the TPS. For the dose reconstruction model, the specific treatment machine parameters are not modeled in the classical sense by pre-defining all geometric and dosimetric parameters of the treatment machine, but the energy fluence delivered by the treatment machine is measured using the EPID as described in Chapter 3. This verification can detect errors in the TPS that occur due to inaccuracies in the modeled parameters. An example of such a deviation was shown in Chapters 4 and 5, where the transmission values through the jaws were directly measured with the EPID and used in the dose reconstruction model. A difference between measured and planned energy fluence was present due to an erroneous implementation of the characteristics of the MLC and collimator jaws of the treatment machine in the version of the TPS used at that time. These differences are quantified in the dose distribution inside a phantom or patient, where a higher dose was reconstructed behind the closed leaves of the MLC. Other groups also reported erroneous modeling of the treatment machine in the TPS. McDermott et al. ${ }^{7}$ detected, with 2D EPID dosimetry, an underdosage in the junction of abutting segments due to a wrong setting of the tongue-and-groove parameter of the MLC in the TPS. Jang et al. ${ }^{17}$ also found and quantified differences between TPS calculated and Monte Carlo simulated IMRT dose distributions, where the TPS underestimated the dose in the low-dose regions caused by inadequate transmission modeling and a simplistic description of the MLC.

The other main factor that determines the dose calculation accuracy is the energy deposition algorithm. Frequently applied dose calculation algorithms in a TPS include the fast, but less accurate, pencil beam or convolution algorithms and the slower, but more accurate, superposition or collapsed cone algorithms. ${ }^{18}$ The dose calculation algorithm of the reconstruction model presented and used in this thesis is adapted from the Monte Carlo code $\mathrm{XVMC}^{19}$. Monte Carlo simulations are, if implemented properly, the most accurate methods for calculating dose currently available. ${ }^{18,20}$ Chapter 4 shows an analysis of the dose distributions calculated using the convolution and superposition algorithm of our clinically applied TPS and compared measured and reconstructed dose distribution for phantoms having various types of geometry and inhomogeneities. The convolution algorithm shows (known) problems with calculating the dose at tissue interfaces such as the lung-tissue interface, or air cavities in the head-and-neck region. Except for some small regions inside the bony structures, the accuracy of the dose calculation of the superposition algorithm implemented in the TPS was in good agreement with the Monte Carlo based dose reconstruction algorithm. Chapter 5 quantifies differences in terms of dose-volume histogram parameters for actual patient treatments in the thorax and head-and-neck region. In the thorax, the convolution algorithm overestimates the mean dose in the target volume with on average $5 \%$ and results in decreased dose homogeneity in the target volume indicated by a decrease in $D_{95 \%}$ of on average $9 \%$. For the superposition algorithm these numbers are below $2 \%$, hence superposition algorithms are necessary for calculating accurate dose distributions in the thorax region. For the IMRT treatments in our institute, dose distributions are planned only using the superposition algorithm and mean differences in dose-volume histogram parameters of the normal tissue such as the maximum dose in the spinal cord or the mean dose in the parotids were generally below $3 \%$. For the target volume the $D_{5 \%}$, mean dose and $D_{95 \%}$ were within $2 \%$ of the planned values. 
In Chapter 6, the importance and implications of using accurate dose calculation algorithms for treatment planning in case of large inhomogeneous regions such as the thorax are discussed. If the dose calculation algorithm in the TPS is switched from the simple convolution to the more advanced and precise superposition algorithm, this may result in differences in tumor dose and a decrease of tumor coverage. In our clinically applied dose-escalation protocol, where the tumor dose is restricted by the normal tissue complications and enlarging field sizes to achieve adequate tumor coverage will result in too large normal tissue toxicity, accurate dose calculation algorithms are important. A strategy is provided to reach a higher total tumor dose by slightly reducing tumor coverage in selected areas, while keeping an iso-toxic treatment. Accurate dose calculations are not only important for the target volume, but also for the normal tissue. For example, Stroian et al. ${ }^{21}$ showed in a retrospective study that a more advanced dose calculation based on Monte Carlo simulations correlated better with radiation-induced fibrosis in the lung than the simple planned dose distribution of the TPS.

\section{Imaging during treatment}

Various studies have been described in the literature that perform imaging during a course of treatments using repeated CT imaging to assess the impact of, either geometric or dosimetric, changes in the patient anatomy. ${ }^{22-27}$ Differences in dose distribution due to a change in anatomy during treatment compared to the moment the planning CT scan was made, may then be quantified in this newly acquired imaging data-set. However, the use of repeated CT imaging creates additional workload, requires an additional time-slot on the conventional CT scanner and care must be taken in repositioning the patient as set-up on the treatment machine. The ability nowadays with cone-beam CT imaging on the treatment machine eliminates the above-mentioned problems; imaging can be performed just prior to or after a treatment fraction. Currently two types of cone-beam $\mathrm{CT}$ imaging modalities are available: kilovoltage $(\mathrm{kV})^{28}$ and megavoltage $(\mathrm{MV})^{29}$ cone-beam CT.

Various approaches are possible to use cone-beam CT information to estimate the actual dose distribution in an irradiated patient. For instance, if the measured set-up errors are not too large, small translations of the patient might be sufficient to guarantee the correct dose delivery. Another way to use cone-beam CT imaging for dose verification purposes, is to perform a calibration of these scanners in terms of electron density and to use this data for an (independent) dose calculation. There are two main effects hampering a calibration of cone-beam CT scanners. The first is the radiation scattered from the phantom or patient reaching the flat panel detector and deteriorating the image. The second phenomenon is that beam hardening as well as the nonuniform spectral response of the flat-panel imager distorts the logarithmic attenuation relation between object thickness and transmitted signal. For the MV cone-beam CT images, a correction procedure is described in Chapter 7 that enables the use of MV cone-beam CT data for dose calculation, either in the TPS or using the 3D dose reconstruction algorithm. For kV cone-beam CT imaging, the effects of scatter and beam hardening are more difficult to model. In kV cone-beam CT imaging scatter-to-primary ratios can be as large as $120 \%{ }^{30}$ depending on the size of the imaged object, air gap 
and field-of-view. Up to now, some researchers investigated correction strategies by correcting the images of the scanner using a calibration measurement with a phantom that has inserts of known electron density. ${ }^{31-33}$ Such an approach is less suitable if deviations between the dimensions of the imaged objects and the calibration measurements occur. Others have performed scatter correction strategies ${ }^{34-36}$, estimation of the cupping by performing measurements with objects of similar dimensions as the imaged object ${ }^{37,38}$ or a registration of the cone-beam CT scan with the planning CT scan that serves as a look-up table for the correct electron density value. ${ }^{39}$

Another important issue is image quality. For both the kV and the MV cone-beam CT scanner, the image quality, e.g. signal-to-noise and contrast-to-noise ratio, is less compared to images acquired using a conventional CT scanner. This is a result of the principle of cone-beam acquisition: a large portion of the radiation that reaches the detector is scattered in the imaged object and hence deteriorates the images. Comparing the two cone-beam CT modalities, MV cone-beam CT has a lower image quality compared to $\mathrm{kV}$ cone-beam $\mathrm{CT}^{40}$ This is mainly caused by the different interaction properties in the MeV range (Compton scattering) compared to the keV range (photoelectric effect) and the lower quantum efficiency of the detectors for photons with energies in the MeV range. Hence, tissue segmentation is more difficult in MV conebeam $\mathrm{CT}$ images compared to $\mathrm{kV}$ imaging for organs having roughly the same electron density. For example, segmentation of the prostate and surrounding tissues in the abdomen region may already be difficult in conventional or kV cone-beam CT imaging, in MV cone-beam CT images this task will even be harder. For tumors in high contrast regions such as the thorax, this is less of a problem. In the head-and-neck region, the bony anatomy and air cavities also create sufficient contrast in identifying the different regions of interest. Identification of structures of interest can be eased and fastened by using the planning CT scan that usually has all organs already delineated. If a conebeam CT scan is taken early during treatment and no large anatomical differences are expected, these structures delineated on the planning CT scan may be copied to the cone-beam CT data set. At a later stage in the treatment, the possibility of changes in anatomy increase and the cone-beam CT data set needs to be manually delineated or more sophisticated tools, such as deformable image registration, are necessary to identify structures of interest by using atlas based segmentation ${ }^{41,42}$ or a deformation of the original delineated structures of the planning CT $\operatorname{scan}^{43-45}$.

\section{D in vivo dosimetry}

The previous sections have shown that the dose in the target volume or the dose in the normal tissues can differ from the planned dose distribution either due to differences between intended and actual treatment delivery or changes in patient anatomy. Hence, for 3D in vivo dosimetry, a combination of 3D in-room imaging as well as a dose measurement during treatment is necessary.

Only a few procedures are described in the literature that combine 3D imaging and dosimetry during treatment. A proof-of-principle study was performed by Partridge et al. $^{46}$, but due to the (too) high dose necessary at that time for acquiring a 3D MV conebeam CT scan, no patient results or clinical studies were performed. With the introduction of low-dose MV cone-beam CT imaging by Pouliot et al. ${ }^{29}$, the dose levels were 
acceptable for (routine) patient imaging. In a review paper on dose-guided radiotherapy by Chen et al. ${ }^{47}$, a single patient case was presented where cone-beam imaging was used to reconstruct dose distributions based on the energy fluence measured behind the patient. However, the dose reconstruction model they used was sub-optimal and did not include corrections for radiation scattered to the EPID and hence the reconstructed dose was $10 \%$ higher than planned. In Chapter 8 , we described a method that reconstructs the 3D dose distribution inside a MV cone-beam CT scan with an error in dose smaller than $3 \%$ for most of the points in the 3D dose distribution. Because differences are quantified in the actual patient anatomy and allow quantification of the dose in the PTV and normal tissue dose, this 3D dose reconstruction is ideally suitable as a verification procedure during a single treatment fraction thereby checking large parts of the radiotherapy process including dose delivery, dose calculation, patient set-up and changes in the patient anatomy compared the planning situation.

$3 \mathrm{D}$ in vivo dosimetry is useful for both retrospective and prospective evaluation of the dose delivered during a course of treatment. Retrospective studies may focus on the outcome of the treatment in relation with the dose delivered to a patient. For example, tumor recurrence can be evaluated in terms of adequate dose coverage of the tumor, or normal tissue complications may be explained by assessing the dose distribution in a specific organ. Prospective evaluation can be undertaken to monitor and assure correct dose delivery during single or multiple fractions of a complete course of treatment. A possible verification strategy could be to perform 3D in vivo dosimetry during the first week of treatment, where the assumption is that the patient anatomy resembles the anatomy at the time of making the planning CT scan. In this way, the entire chain in radiotherapy from acquiring planning $\mathrm{CT}$ data, treatment plan design and treatment delivery is verified. Another verification strategy could be to apply 3D in vivo dosimetry later during the patient treatment, for example weekly or halfway through the complete course. 3D in vivo dosimetry is then used to prospectively monitor the quality of the treatment delivery, taking possible changes in anatomy into account. Verification is performed whether tumor coverage is still sufficient or organs at risk are reaching their normal tissue toxicity, the treatment plan can be reevaluated and possible adjustments of the treatment plan may be performed.

\section{EXTENDING ART: FROM IGRT TO DGRT}

\section{Image-guided radiotherapy}

With the introduction of intensity-modulated radiotherapy ${ }^{48,49}$, dose distributions can now be created that have complex concave shapes and steep dose gradients resulting in a better sparing of organs at risk, which was not possible with 3D conformal radiotherapy.

In order to exploit the full potential of IMRT, it is crucial that patient positioning is more accurate and reproducible than it is for 3D conformal radiotherapy. Imageguided radiotherapy fills in this part and has in many clinics been introduced almost together with IMRT. Often daily images of the patient are made using in-room imaging 
modalities, e.g. EPID, cone-beam CT, CT-on-rails, stereoscopic kV fluoroscopy or ultrasound localization, and allow repositioning of the patient on a daily basis. The actual anatomy is imaged and aligned with the anatomy that was used during the design of the treatment plan, i.e. usually the planning CT scan. With IGRT, high-precision patient positioning is available and necessary to make optimal use of the possible advantage of IMRT.

\section{Adaptive radiotherapy}

Image information acquired with IGRT during treatment is currently used for patient positioning and alignment. The next step is to use this information as a starting point for adjustments or adaptation of the treatment. This procedure is often referred to as adaptive radiotherapy (ART). ${ }^{50}$ Some institutes started protocols and studies to use the recent development of in-room imaging capabilities to tailor the treatment to make optimal use of this information. For example in prostate cancer, the most frequent or mean position of the prostate after the first few (e.g. five) fractions can be determined and the plan is adapted based on this new position. ${ }^{51-53}$ Others have investigated the use of a daily re-optimization of the treatment plan $^{54}$ based on the new positions of the organ or described methods to adapt the leaf positions of the MLC to the current position of the prostate and seminal vesicles ${ }^{55}$. The basis of this type of image-guided ART is the frequent (on-line) imaging possibilities nowadays available in a radiotherapy department and adaptation of the treatment plan based on the changes observed in the images.

\section{Dose-guided radiotherapy}

With IGRT, the position of the tumor and organs at risk during treatment is determined. However, not only geometric aspects of a treatment should be verified, because the aim of radiotherapy is to deliver a high dose to the tumor while sparing the surrounding tissue. The incorporation of the actual dose delivered during multiple treatment fractions should form the basis for making well-defined adjustments of the treatment plan for an individual patient. Dose-guided radiotherapy (DGRT) can be seen as the next-step of the adaptive radiotherapy process. Figure 9.1 shows the difference in approaches of IGRT and DGRT for adaptive radiotherapy. With DGRT, the dose delivery is also part of the feedback loop. By incorporating both the actual dose and the actual anatomy (IGRT) of the patient for every fraction, a comprehensive evaluation of the dose delivered to the target volume and normal tissue is possible.

For this purpose, new strategies and techniques are necessary for the evaluation of these multiple dose and anatomy datasets during the course of treatment. One of the techniques to take into account changes in patient anatomy is the use of deformable (i.e. non-rigid) registration. ${ }^{45,56,57}$ This type of registration is necessary to make a relation between the various datasets and to 'link' all structures of different modalities or time points to each other. In other words a specific voxel or group of voxels (e.g. the tumor or an organ at risk) can be followed over time and the actual dose delivered to a single or group of voxels can be accumulated. This technique is still in its early stage and lacks clinical validation; answers need to be found on how anatomy changes, 


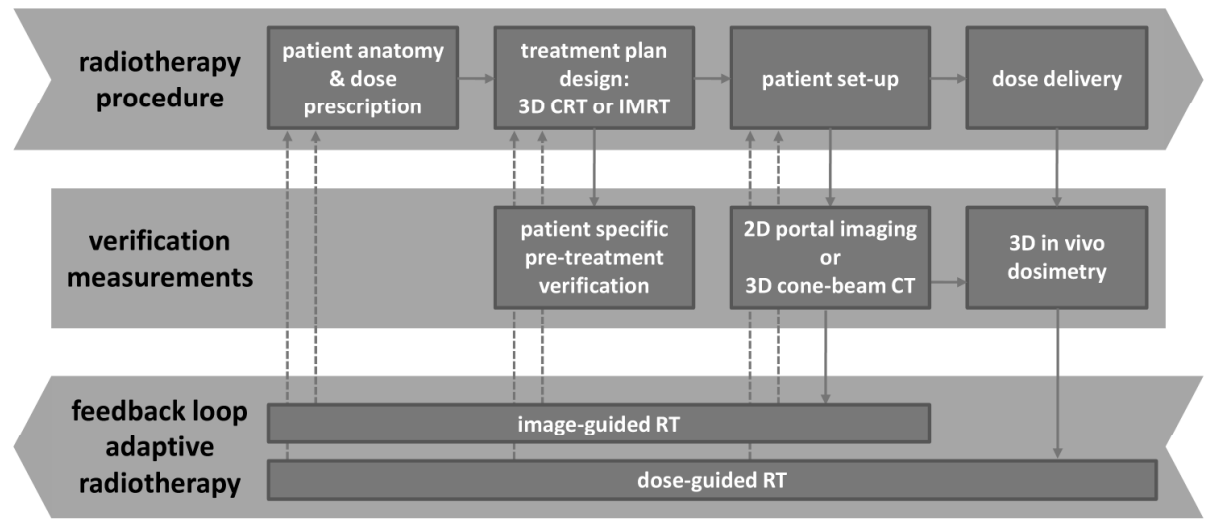

Figure 9.1: Schematic overview of the radiotherapy (RT) process with the position of the verification measurements and the adaptive radiotherapy feedback loops: image-guided RT and doseguided $R T$.

induced by the therapy, need to be taken into account, e.g. what to do with disappearing voxels due to effect of radiotherapy, or tumor growth and shrinkage. For the normal-tissue this might be less of a problem because generally no large (volume) changes are expected because of the sparing of these organs from the high dose regions, although tumor shrinkage can cause the normal tissue to be exposed to a higher than expected dose. Various groups already applied DGRT strategies in clinical practice and estimated the dose to the normal tissues during treatment, for example the dose in the parotids in the head-and-neck region. ${ }^{27,44}$ Others investigated techniques for compensation of loss in tumor coverage by repairing the lack of dose in an additional treatment fraction at the end or by a compensation in the remaining fractions. ${ }^{58}$ Dose-guided radiotherapy techniques still need to be further developed and studies are required to assess the added value in clinical practice.

\section{FUTURE PERSPECTIVES}

The methods described in this thesis form a solid basis for future research in many areas. First of all for clinical studies, the actual cumulative dose delivered for an entire course of radiotherapy may be valuable information for many clinical investigations. E.g. immobilization devices, treatment class solutions and delivery techniques, fractionation strategies or set-up protocols may be evaluated in terms of delivered dose.

Extension of the current DGRT frame-work is necessary to take into account intrafraction motion of the tumor and normal tissue. For example, a tumor in the lung may move because of the respiratory cycle. A standard technique for measuring tumor excursion is by 4D CT imaging. However, dose delivery at the treatment machine may be applied in a single phase using gating procedures, but usually free-breathing is allowed and the tumor position may move in the respiratory cycle. To fully determine 
the delivered dose distribution, the model should then be extended at various parts to take into account these changes in anatomy. First, 4D imaging (e.g. 4D cone-beam CT) should be performed where the different phases of the intra-fraction motion are captured. Second, the measurement of the energy fluence should be binned to the corresponding phases of the anatomy and, third, the dose should be reconstructed in each of these phases. By using deformable image registration the dose in the various phases can be registered to a reference phase for evaluation of the dose delivery.

Another area of research that is possible, using the frame-work of DGRT, is the characterization of dose-response of specific voxels inside the target volume or normal tissue. DGRT allows a measurement of the dose delivered to individual voxels inside a patient. Using (deformable) image registration, the dose inside this voxel can be followed during the course of treatment. The chance that a voxel receives the correct dose and that the tumor tissue inside is destroyed, or the chance that the normal tissue will be damaged and cause complications or failure of its function is called: voxel control or complication probability (VCP). ${ }^{59,60}$ By incorporating imaging modalities (e.g. PET or MRI) and biological features, e.g. tumor cell characteristics such as hypoxia, clonogenic cell density, and proliferation rates or for the normal tissue specific functional properties, a true VCP model may be derived for specific tumors or normal tissues. By combining the VCP concept with boosting or dose-redistribution strategies, higher control rates or reduced normal tissue toxicity may be achieved. ${ }^{61,62}$

The dose delivered to the patient may be incorporated in retrospective analyses and incorporated by adding a quality measurement of the dose delivery into the analysis. The implicit assumption that treatment is delivered as planned in the evaluating of treatment response adds a large uncertainty that has to be removed to further improve the prediction models in the future. By adding dosimetry information to predictive models, the dose delivered to the patient will then be one parameter of the large set of predictive parameters for treatment response. The development of such predictive models is currently under investigation and named computer assisted theragnostics (CAT). ${ }^{63-65}$

\section{CLINICAL IMPLEMENTATION OF 3D DOSIMETRY: A TWO-FOLD PURPOSE}

To summarize, the models developed and described in this thesis have two main applications in the field of radiotherapy. The first one is that they are able to verify the actual dose delivered to the patient, in a pre-treatment as well as in an actual treatment (in vivo) setting. The pre-treatment stage has been developed to assure that the planned (expected) dose is in agreement with the dose that actually will be delivered to the patient (Chapters 3-6). The in vivo stage is used to assess the impact in terms of dose of possible changes in anatomy acquired using in-room imaging (Chapter 7) as well as possible differences in treatment delivery (Chapter 8): a reconstruction of the actual dose delivered during a specific treatment fraction is possible. For retrospective studies of outcome and effectiveness of treatment, these methods can be of great importance. The actual quality of the treatment can be taken into account and re- 
moves the uncertainty that is inherently present in all retrospective models and investigations nowadays: a possible discrepancy between planned and actual dose delivered to the patient.

The second strategy that uses the methods described in this thesis is dose-guided adaptive radiotherapy. With 3D dose reconstruction, the dose delivered during treatment can be followed and used prospectively. Differences in tumor shape and volume changes, patient anatomy changes, or dose delivery differences compared to the planned dose, are quantified and can be taken into account or compensated. True dose-guided radiotherapy is to incorporate all the information gathered during treatment to be used in the remaining course of treatment. Because radiotherapy is generally delivered in many fractions, there is room for updating and adapting the treatment plan to the changing situation. Dose-guided radiotherapy and adaptive radiotherapy are key strategies that will be applied in the future in the context of a continuing personalized and individualized treatment.

Clinical experience is based on evidence-based medicine carefully acquired in clinical trials; one has therefore to be cautious with a fast implementation of this second approach because navigating away from the known treatment technique will require a thorough investigation and detailed (long-term) follow-up. However, because in some patients radiotherapy is failing and side-effects do occur, there is still room for improvement. 3D dose verification for evaluation of the dose delivered and, in the next step, adaptation of the treatment based on the dose distribution delivered may be a critical step towards further improvement of radiotherapy.

\section{REFERENCES}

${ }^{1} \mathrm{E}$. Yorke, R. Alecu, L. Ding, and et al, Diode in vivo dosimetry for patients receiving external beam radiation therapy. AAPM Report No. 87, Task Group 62 of the AAPM Radiation Therapy Committee. 2005, Madison, WI: Medical Physics Publishing.

${ }^{2} \mathrm{M}$. Essers and B.J. Mijnheer, "In vivo dosimetry during external photon beam radiotherapy," Int J Radiat Oncol Biol Phys 43, 245-259 (1999).

${ }^{3}$ B.M. Hesse, S. Nill, T. Tücking, and U. Oelfke, "3D in vivo dosimetry by means of entrance dosimetry for dose guided radiotherapy," Radiother Oncol 81, S46 (2006).

${ }^{4}$ B. Poppe, C. Thieke, D. Beyer, R. Kollhoff, A. Djouguela, A. Ruhmann, K.C. Willborn, and D. Harder, "DAVID--a translucent multi-wire transmission ionization chamber for in vivo verification of IMRT and conformal irradiation techniques," Phys Med Biol 51, 1237-1248 (2006).

${ }^{5}$ S.C. Vieira, M.L. Dirkx, B.J. Heijmen, and H.C. de Boer, "SIFT: a method to verify the IMRT fluence delivered during patient treatment using an electronic portal imaging device," Int J Radiat Oncol Biol Phys 60, 981-993 (2004).

${ }^{6}$ L.N. McDermott, M. Wendling, J.J. Sonke, M. van Herk, and B.J. Mijnheer, "Replacing pretreatment verification with in vivo EPID dosimetry for prostate IMRT," Int J Radiat Oncol Biol Phys 67, 1568-1577 (2007).

${ }^{7}$ L.N. McDermott, M. Wendling, B. van Asselen, J. Stroom, J.J. Sonke, M. van Herk, and B.J. Mijnheer, "Clinical experience with EPID dosimetry for prostate IMRT pre-treatment dose verification," Med Phys 33, 3921-3930 (2006).

${ }^{8} \mathrm{M}$. Wendling, R.J. Louwe, L.N. McDermott, J.J. Sonke, M. van Herk, and B.J. Mijnheer, "Accurate two-dimensional IMRT verification using a back-projection EPID dosimetry method," Med Phys 33, 259-273 (2006). 
${ }^{9}$ R. Boellaard, M. van Herk, H. Uiterwaal, and B. Mijnheer, "First clinical tests using a liquid-filled electronic portal imaging device and a convolution model for the verification of the midplane dose," Radiother Oncol 47, 303-312 (1998).

${ }^{10}$ W.J. van Elmpt, S.M. Nijsten, B.J. Mijnheer, and A.W. Minken, "Experimental verification of a portal dose prediction model," Med Phys 32, 2805-2818 (2005).

${ }^{11}$ M. Kroonwijk, K.L. Pasma, S. Quint, P.C. Koper, A.G. Visser, and B.J. Heijmen, "In vivo dosimetry for prostate cancer patients using an electronic portal imaging device (EPID); demonstration of internal organ motion," Radiother Oncol 49, 125-132 (1998).

${ }^{12}$ K.L. Pasma, B.J. Heijmen, M. Kroonwijk, and A.G. Visser, "Portal dose image (PDI) prediction for dosimetric treatment verification in radiotherapy. I. An algorithm for open beams," Med Phys 25, 830-840 (1998).

${ }^{13}$ K.L. Pasma, S.C. Vieira, and B.J. Heijmen, "Portal dose image prediction for dosimetric treatment verification in radiotherapy. II. An algorithm for wedged beams," Med Phys 29, 925931 (2002).

${ }^{14}$ S.M. Nijsten, B.J. Mijnheer, A.L. Dekker, P. Lambin, and A.W. Minken, "Routine individualised patient dosimetry using electronic portal imaging devices," Radiother Oncol 83, 65-75 (2007).

${ }^{15}$ A. Van Esch, T. Depuydt, and D.P. Huyskens, "The use of an aSi-based EPID for routine absolute dosimetric pre-treatment verification of dynamic IMRT fields," Radiother Oncol 71, 223234 (2004).

${ }^{16}$ S.M. Nijsten, W.J. van Elmpt, B. Mijnheer, L. Persoon, P. Lambin, and A. Dekker, "2D in-vivo verification in clinical routine using EPID dosimetry: clinical experience with more than 2000 patients," Radiother Oncol ESTRO conference 2008, Göteborg, Sweden (2008).

${ }^{17}$ S.Y. Jang, H.H. Liu, and R. Mohan, "Underestimation of low-dose radiation in treatment planning of intensity-modulated radiotherapy," Int J Radiat Oncol Biol Phys 71, 1537-1546 (2008).

${ }^{18}$ N. Papanikolaou, J. Battista, A. Boyer, C. Kappas, E. Klein, T.R. Mackie, M. Sharpe, and J. Van Dyk, Tissue inhomogeneity corrections for megavoltage photon beams. AAPM Report No. 85, Task Group No. 65 of the Radiation Therapy Committee of the American Association of Physicist in Medicine. 2004, Madison, WI: Medical Physics Publishing.

${ }^{19} \mathrm{M}$. Fippel, "Fast Monte Carlo dose calculation for photon beams based on the VMC electron algorithm," Med Phys 26, 1466-1475 (1999).

${ }^{20}$ I.J. Chetty, B. Curran, J.E. Cygler, J.J. DeMarco, G. Ezzell, B.A. Faddegon, I. Kawrakow, P.J. Keall, H. Liu, C.M. Ma, D.W. Rogers, J. Seuntjens, D. Sheikh-Bagheri, and J.V. Siebers, "Report of the AAPM Task Group No. 105: Issues associated with clinical implementation of Monte Carlo-based photon and electron external beam treatment planning," Med Phys 34, 48184853 (2007).

${ }^{21}$ G. Stroian, C. Martens, L. Souhami, D.L. Collins, and J. Seuntjens, "Local correlation between monte-carlo dose and radiation-induced fibrosis in lung cancer patients," Int J Radiat Oncol Biol Phys 70, 921-930 (2008).

${ }^{22}$ C. Lee, K.M. Langen, W. Lu, J. Haimerl, E. Schnarr, K.J. Ruchala, G.H. Olivera, S.L. Meeks, P.A. Kupelian, T.D. Shellenberger, and R.R. Manon, "Evaluation of geometric changes of parotid glands during head and neck cancer radiotherapy using daily MVCT and automatic deformable registration," Radiother Oncol Epub Aug 14 (2008).

${ }^{23}$ G. Bosmans, A. van Baardwijk, A. Dekker, M. Ollers, L. Boersma, A. Minken, P. Lambin, and D. De Ruysscher, "Intra-patient variability of tumor volume and tumor motion during conventionally fractionated radiotherapy for locally advanced non-small-cell lung cancer: a prospective clinical study," Int J Radiat Oncol Biol Phys 66, 748-753 (2006). 
${ }^{24}$ P.A. Kupelian, C. Ramsey, S.L. Meeks, T.R. Willoughby, A. Forbes, T.H. Wagner, and K.M. Langen, "Serial megavoltage CT imaging during external beam radiotherapy for non-smallcell lung cancer: observations on tumor regression during treatment," Int J Radiat Oncol Biol Phys 63, 1024-1028 (2005).

${ }^{25}$ J.C. Roeske, J.D. Forman, C.F. Mesina, T. He, C.A. Pelizzari, E. Fontenla, S. Vijayakumar, and G.T. Chen, "Evaluation of changes in the size and location of the prostate, seminal vesicles, bladder, and rectum during a course of external beam radiation therapy," Int J Radiat Oncol Biol Phys 33, 1321-1329 (1995).

${ }^{26}$ E.K. Hansen, M.K. Bucci, J.M. Quivey, V. Weinberg, and P. Xia, "Repeat CT imaging and replanning during the course of IMRT for head-and-neck cancer," Int J Radiat Oncol Biol Phys 64, 355-362 (2006).

${ }^{27}$ C. Lee, K.M. Langen, W. Lu, J. Haimerl, E. Schnarr, K.J. Ruchala, G.H. Olivera, S.L. Meeks, P.A. Kupelian, T.D. Shellenberger, and R.R. Manon, "Assessment of parotid gland dose changes during head and neck cancer radiotherapy using daily megavoltage computed tomography and deformable image registration," Int J Radiat Oncol Biol Phys 71, 1563-1571 (2008).

${ }^{28}$ D.A. Jaffray, J.H. Siewerdsen, J.W. Wong, and A.A. Martinez, "Flat-panel cone-beam computed tomography for image-guided radiation therapy," Int J Radiat Oncol Biol Phys 53, 13371349 (2002).

${ }^{29}$ J. Pouliot, A. Bani-Hashemi, J. Chen, M. Svatos, F. Ghelmansarai, M. Mitschke, M. Aubin, P. Xia, O. Morin, K. Bucci, M. Roach, 3rd, P. Hernandez, Z. Zheng, D. Hristov, and L. Verhey, "Lowdose megavoltage cone-beam CT for radiation therapy," Int J Radiat Oncol Biol Phys 61, 552-560 (2005).

${ }^{30}$ J.H. Siewerdsen and D.A. Jaffray, "Cone-beam computed tomography with a flat-panel imager: magnitude and effects of x-ray scatter," Med Phys 28, 220-231 (2001).

${ }^{31}$ Y. Yang, E. Schreibmann, T. Li, C. Wang, and L. Xing, "Evaluation of on-board kV cone beam CT (CBCT)-based dose calculation," Phys Med Biol 52, 685-705 (2007).

${ }^{32}$ S. Yoo and F.F. Yin, "Dosimetric feasibility of cone-beam CT-based treatment planning compared to CT-based treatment planning," Int J Radiat Oncol Biol Phys 66, 1553-1561 (2006).

${ }^{33}$ D. Letourneau, R. Wong, D. Moseley, M.B. Sharpe, S. Ansell, M. Gospodarowicz, and D.A. Jaffray, "Online planning and delivery technique for radiotherapy of spinal metastases using cone-beam CT: image quality and system performance," Int J Radiat Oncol Biol Phys 67, 1229-1237 (2007).

${ }^{34}$ G. Jarry, S.A. Graham, D.J. Moseley, D.J. Jaffray, J.H. Siewerdsen, and F. Verhaegen, "Characterization of scattered radiation in kV CBCT images using Monte Carlo simulations," Med Phys 33, 4320-4329 (2006).

${ }^{35}$ J. Rinkel, L. Gerfault, F. Esteve, and J.M. Dinten, "A new method for x-ray scatter correction: first assessment on a cone-beam CT experimental setup," Phys Med Biol 52, 4633-4652 (2007).

${ }^{36}$ J.H. Siewerdsen, M.J. Daly, B. Bakhtiar, D.J. Moseley, S. Richard, H. Keller, and D.A. Jaffray, "A simple, direct method for x-ray scatter estimation and correction in digital radiography and cone-beam CT," Med Phys 33, 187-197 (2006).

${ }^{37}$ O. Morin, J. Chen, M. Aubin, A. Gillis, J.F. Aubry, S. Bose, H. Chen, M. Descovich, P. Xia, and J. Pouliot, "Dose calculation using megavoltage cone-beam CT," Int J Radiat Oncol Biol Phys 67, 1201-1210 (2007).

${ }^{38}$ M. Kachelriess, K. Sourbelle, and W.A. Kalender, "Empirical cupping correction: a first-order raw data precorrection for cone-beam computed tomography," Med Phys 33, 1269-1274 (2006).

${ }^{39}$ M. van Zijtveld, M. Dirkx, and B. Heijmen, "Correction of conebeam CT values using a planning CT for derivation of the "dose of the day"," Radiother Oncol 85, 195-200 (2007). 
${ }^{40}$ B.A. Groh, J.H. Siewerdsen, D.G. Drake, J.W. Wong, and D.A. Jaffray, "A performance comparison of flat-panel imager-based MV and kV cone-beam CT," Med Phys 29, 967-975 (2002).

${ }^{41}$ O. Commowick, V. Gregoire, and G. Malandain, "Atlas-based delineation of lymph node levels in head and neck computed tomography images," Radiother Oncol 87, 281-289 (2008).

${ }^{42}$ T. Zhang, Y. Chi, E. Meldolesi, and D. Yan, "Automatic delineation of on-line head-and-neck computed tomography images: toward on-line adaptive radiotherapy," Int J Radiat Oncol Biol Phys 68, 522-530 (2007).

${ }^{43}$ W. Lu, G.H. Olivera, Q. Chen, K.J. Ruchala, J. Haimerl, S.L. Meeks, K.M. Langen, and P.A. Kupelian, "Deformable registration of the planning image ( $\mathrm{kVCT}$ ) and the daily images (MVCT) for adaptive radiation therapy," Phys Med Biol 51, 4357-4374 (2006).

${ }^{44}$ J.C. O'Daniel, A.S. Garden, D.L. Schwartz, H. Wang, K.K. Ang, A. Ahamad, D.I. Rosenthal, W.H. Morrison, J.A. Asper, L. Zhang, S.M. Tung, R. Mohan, and L. Dong, "Parotid gland dose in intensity-modulated radiotherapy for head and neck cancer: is what you plan what you get?," Int J Radiat Oncol Biol Phys 69, 1290-1296 (2007).

${ }^{45}$ P. Castadot, J.A. Lee, A. Parraga, X. Geets, B. Macq, and V. Gregoire, "Comparison of 12 deformable registration strategies in adaptive radiation therapy for the treatment of head and neck tumors," Radiother Oncol (2008).

${ }^{46}$ M. Partridge, M. Ebert, and B.M. Hesse, "IMRT verification by three-dimensional dose reconstruction from portal beam measurements," Med Phys 29, 1847-1858 (2002).

${ }^{47}$ J. Chen, O. Morin, M. Aubin, M.K. Bucci, C.F. Chuang, and J. Pouliot, "Dose-guided radiation therapy with megavoltage cone-beam CT," Br J Radiol 79 Spec No 1, S87-98 (2006).

${ }^{48}$ Intensity Modulated Radiation Therapy Collaborative Working Group, "Intensity-modulated radiotherapy: current status and issues of interest," Int J Radiat Oncol Biol Phys 51, 880914 (2001).

${ }^{49}$ G.A. Ezzell, J.M. Galvin, D. Low, J.R. Palta, I. Rosen, M.B. Sharpe, P. Xia, Y. Xiao, L. Xing, and C.X. $\mathrm{Yu}$, "Guidance document on delivery, treatment planning, and clinical implementation of IMRT: report of the IMRT Subcommittee of the AAPM Radiation Therapy Committee," Med Phys 30, 2089-2115 (2003).

${ }^{50}$ D. Yan, F. Vicini, J. Wong, and A. Martinez, "Adaptive radiation therapy," Phys Med Biol 42, 123-132 (1997).

${ }^{51}$ J. Nijkamp, F.J. Pos, T.T. Nuver, R. de Jong, P. Remeijer, J.J. Sonke, and J.V. Lebesque, "Adaptive radiotherapy for prostate cancer using kilovoltage cone-beam computed tomography: first clinical results," Int J Radiat Oncol Biol Phys 70, 75-82 (2008).

${ }^{52}$ T.T. Nuver, M.S. Hoogeman, P. Remeijer, M. van Herk, and J.V. Lebesque, "An adaptive off-line procedure for radiotherapy of prostate cancer," Int J Radiat Oncol Biol Phys 67, 1559-1567 (2007).

${ }^{53}$ D. Yan, D. Lockman, D. Brabbins, L. Tyburski, and A. Martinez, "An off-line strategy for constructing a patient-specific planning target volume in adaptive treatment process for prostate cancer," Int J Radiat Oncol Biol Phys 48, 289-302 (2000).

${ }^{54}$ Q.J. Wu, D. Thongphiew, Z. Wang, B. Mathayomchan, V. Chankong, S. Yoo, W.R. Lee, and F.F. Yin, "On-line re-optimization of prostate IMRT plans for adaptive radiation therapy," Phys Med Biol 53, 673-691 (2008).

${ }^{55}$ L.E. Court, L. Dong, A.K. Lee, R. Cheung, M.D. Bonnen, J. O'Daniel, H. Wang, R. Mohan, and D. Kuban, "An automatic CT-guided adaptive radiation therapy technique by online modification of multileaf collimator leaf positions for prostate cancer," Int J Radiat Oncol Biol Phys 62, 154-163 (2005).

${ }^{56}$ J. Orban de Xivry, G. Janssens, G. Bosmans, M. De Craene, A. Dekker, J. Buijsen, A. van Baardwijk, D. De Ruysscher, B. Macq, and P. Lambin, "Tumour delineation and cumulative dose computation in radiotherapy based on deformable registration of respiratory correlated CT images of lung cancer patients," Radiother Oncol 85, 232-238 (2007). 
${ }^{57}$ M. Sohn, M. Birkner, Y. Chi, J. Wang, Y. Di, B. Berger, and M. Alber, "Model-independent, multimodality deformable image registration by local matching of anatomical features and minimization of elastic energy," Med Phys 35, 866-878 (2008).

${ }^{58} \mathrm{Q}$. Wu, J. Liang, and D. Yan, "Application of dose compensation in image-guided radiotherapy of prostate cancer," Phys Med Biol 51, 1405-1419 (2006).

${ }^{59}$ P. Lambin, S. Petit, W. van Elmpt, S. Fekkes, B. Wouters, A. Dekker, and D. De Ruysscher, "Radiotherapy 5.0 - Voxel Control Probability Guided Radiotherapy (VCP-GRT): An Attempt to integrate molecular imaging and dose uncertainties with modern radiation dose delivery," Radiother Oncol 88, S300-S301 (2008).

${ }^{60}$ W. van Elmpt, S. Petit, A. Dekker, and P. Lambin, "Voxel Dose Probability: A New Concept for Treatment Planning Incorporating Dose Uncertainties During Treatment Delivery," Int J Radiat Oncol Biol Phys 69, S696 (2007).

${ }^{61}$ S.M. Bentzen, "Dose painting and theragnostic imaging: towards the prescription, planning and delivery of biologically targeted dose distributions in external beam radiation oncology," Cancer Treat Res 139, 41-62 (2008).

${ }^{62}$ C.C. Ling, J. Humm, S. Larson, H. Amols, Z. Fuks, S. Leibel, and J.A. Koutcher, "Towards multidimensional radiotherapy (MD-CRT): biological imaging and biological conformality," Int J Radiat Oncol Biol Phys 47, 551-560 (2000).

${ }^{63}$ C. Dehing, H. van der Weide, S. Wanders, L. Boersma, D. De Ruysscher, B. Nijsten, H. Steck, S. Krishnan, R.B. Rao, and P. Lambin, "Combining Clinical Data With Medical Knowledge Improves Prediction Models for NSCLC After (Chemo) Radiotherapy," Int J Radiat Oncol Biol Phys 66, S482 (2006).

${ }^{64}$ C. Dehing-Oberije, G. Fung, D. De Ruysscher, H. van der Weide, S. Krishnan, R.B. Rao, and P. Lambin, "The Limitations of Dosimetric Parameters for the Prediction of Radiation-Induced Lung Toxicity: An Approach Based on Machine Learning Techniques," Int J Radiat Oncol Biol Phys 69, S488-S489 (2007).

${ }^{65}$ C. Krisnapuram, C. Dehing, H. Steck, H. van der Weide, D. De Ruysscher, S. Nijsten, S. Wanders, L. Boersma, R.B. Rao, and P. Lambin, "A Knowledge-Model for Predicting Radiation-Induced Esophagitis," Int J Radiat Oncol Biol Phys 69, 5624 (2007). 

SUMMARY 



\section{SUMMARY}

In external beam radiotherapy, the goal is to deliver a high dose to the tumor while sparing the normal tissue and nearby organs at risk. Accurate geometric and dosimetric design of the treatment plan is therefore mandatory to avoid damaging healthy tissue and assure adequate coverage of the tumor. In this thesis, models are developed and procedures are described that combine measurements of the beam delivery either during or prior to treatment, with 3D imaging of the anatomy to derive information on the three-dimensional (3D) dose distribution inside the patient.

Three-dimensional dose verification strategies are applied in two different parts of the treatment procedure. A 3D verification strategy prior to treatment (pre-treatment dose verification) and a $3 \mathrm{D}$ verification strategy applied during treatment (in vivo dosimetry). This thesis is divided in two parts dealing with both strategies.

A general introduction elucidating the importance of dose verification procedures in radiation oncology is given in Chapter 1, with a focus on the use of 3D dose verification in advanced radiotherapy. In Chapter 2, a comprehensive overview on the use of electronic portal imaging devices (EPIDs) for dosimetry purposes is presented. In this literature review, the dosimetric properties and characteristics of EPIDs and the calibration procedures are discussed, as well as the use of EPIDs for pre-treatment dose verification and in vivo dosimetry. The type of errors that can be detected with EPID dosimetry and frequently used acceptance and rejection criteria for interpreting the differences observed between planned and measured dose distributions are presented and current clinical applications of EPID dosimetry are summarized.

Chapters 3-5 describe verification methods and procedures that are applied before the first fraction of a treatment is given. These pre-treatment verification strategies are useful in detecting errors made during treatment planning, dose calculation, treatment plan transfer and to quantify differences between planned and actual delivered energy fluence distributions of treatment fields using the planning CT scan of the patient. Chapter 3 presents the model in detail. This model measures the dose delivered by the linear accelerator with an EPID and reconstructs the 3D dose distribution inside a predefined phantom geometry using a Monte Carlo dose calculation. The method was evaluated for homogeneous phantoms and good agreement between 3D reconstructed and measured dose distributions was observed with $99 \%$ of the points within $3 \%$ or $3 \mathrm{~mm}$ of the measured dose distribution, for both $3 \mathrm{D}$ conformal and intensity-modulated treatment fields.

Chapter $\mathbf{4}$ extends the dose reconstruction model to situations having tissue inhomogeneities and has been verified using inhomogeneous phantoms of various dimensions, including anthropomorphic phantoms. A comparison with the 3D dose distribution calculated using the simple convolution and the more advanced superposition algorithm of the clinically applied treatment planning system is performed. The dose reconstruction method shows that the convolution algorithm is inaccurate in calculating the dose at tissue interfaces present near the lung in the thorax region, or near air cavities in the head-and-neck region. The accuracy of the dose calculation of the superposition algorithm implemented in the TPS was in good agreement with the 
Monte Carlo based dose reconstruction algorithm, except for some small regions inside bony structures.

Chapter 5 applies the methods discussed in Chapters 3 and 4 in a clinical setting and an evaluation is presented of actual patient treatment plans with the dose reconstructed inside the planning CT scan for tumors in the head-and-neck and thoracic region. Differences are expressed in terms of dose-volume histogram (DVH) parameters. For the thorax region, treatment plans calculated using the convolution algorithm of the treatment planning system are compared to the 3D reconstructed dose distribution showing a reduction of on average $5 \%$ in the mean target volume dose. In addition a decreased dose homogeneity in the target volume was observed indicated by a decrease in $D_{95 \%}$ of on average $9 \%$. Recalculating these plans using the superposition algorithm reduced these differences below $2 \%$. From these results, it can be concluded that superposition algorithms are necessary for calculating accurate dose distributions in the thorax region. For the head-and-neck cancer patients treated with intensitymodulated radiotherapy (IMRT), differences between calculated dose distribution of the treatment planning system using the superposition algorithm and the 3D reconstructed dose were generally below $3 \%$. For the target volume the $D_{5 \%}$, mean dose and $\mathrm{D}_{95 \%}$ were within $2 \%$ of the planned values and the values of the normal tissue dose were within $3 \%$ of the planned values.

In Chapter 6, the importance and implications of using accurate dose calculation algorithms for designing 3D conformal treatment plans for patients diagnosed with non-small cell lung cancer are discussed. Different treatment plans are designed using both the convolution and the superposition algorithm following a dose-escalation protocol based on normal tissue constraints. Switching the dose calculation algorithm in the TPS from the convolution to the superposition algorithm results in both a lower dose and a decrease of coverage of the target volume. In the dose-escalation protocol, the tumor dose is restricted by the normal tissue complications and an enlargement of the field sizes to achieve adequate tumor coverage will result in too large normal tissue toxicity. A strategy is provided to implement the more advanced dose calculation algorithms that are able to reach a higher total tumor dose by slightly reducing tumor coverage in selected areas, while keeping an iso-toxic treatment.

The second part of this thesis deals with verification procedures that are applied during treatment. For this purpose, it is necessary to have an accurate model of the patient anatomy prior to or just after treatment, with the patient still in treatment position. Chapter 7 describes a method to acquire a megavoltage (MV) cone-beam CT scan in the treatment room and a calibration procedure is developed to use these images for dose calculation purposes. The images are corrected for the cupping artifact and the Hounsfield units are converted into electron density values. This makes these images suitable for dose calculation using the treatment planning system or using Monte Carlo dose calculations.

Chapter 8 combines the methods developed for pre-treatment verification with MV cone-beam CT imaging. The actual delivered treatment fields are captured behind the patient using the EPID and these fields are used in combination with the in-room acquired cone-beam CT scan. The model back-projects the transmission EPID images behind a patient during treatment to reconstruct the 3D dose distribution delivered of that fraction inside the MV cone-beam CT scan of the actual patient anatomy. The 
accuracy was assessed using a phantom study showing that the error in the reconstructed dose was smaller than 3\% for most of the points in the 3D dose distribution. Because differences are quantified in the actual patient anatomy and allow quantification of the dose in the PTV and normal tissue dose, this 3D dose reconstruction is ideally suitable as a verification procedure during a single treatment fraction checking large parts of the radiotherapy process including dose delivery, dose calculation, patient set-up and changes in the patient anatomy compared the planning situation. As a proof of principle, the method was used to verify the dose delivery for a headand-neck cancer patient treated with IMRT. Except for some small regions around air cavities, good agreement was achieved between the reconstructed dose distribution in the MV cone-beam CT scan and the planned dose distribution inside the planning CT scan. The relevant parameters of the DVHs for the normal tissue were within $3 \%$ of the planned values and the reconstructed mean dose in the target volume was within $2 \%$ of the planned value.

Chapter 9 gives a general discussion on the use of 3D pre-treatment verification and $3 \mathrm{D}$ in vivo dosimetry methods for verification of advanced radiotherapy. These models are necessary to extend the framework of adaptive radiotherapy (ART) to incorporate the dose delivery during treatment: dose-guided radiotherapy (DGRT). Future perspectives are described and an outlook is given on how to incorporate these advanced models in an adaptive way during the course of treatment. 

SAMENVATTING 



\section{SAMENVATTING}

Het doel van radiotherapie is het leveren van een hoge stralingsdosis aan de tumor, terwijl de gezonde normale weefsels en organen zoveel mogelijk worden gespaard. Een nauwkeurig dosimetrisch en geometrisch ontwerp van het behandelplan is dus noodzakelijk om beschadiging van gezonde weefsels te voorkomen en te zorgen voor een voldoende stralingsdosis in de tumor. In dit proefschrift zijn modellen ontwikkeld en procedures beschreven die informatie geven over de dosisafgifte, zowel vóóraf als tijdens de behandeling. Deze informatie kan worden gecombineerd met driedimensionale (3D) beeldvorming van de anatomie om informatie over de 3D-dosisverdeling binnen de patiënt te verkrijgen.

3D-dosisverificatiestrategieën kunnen worden toegepast op twee verschillende tijdspunten gedurende een behandeling. Een 3D-dosisverificatie voorafgaand aan de behandeling ("pre-treatment" dosimetrie), en een 3D-verificatiestrategie toegepast tijdens de behandeling (in vivo dosimetrie). Dit proefschrift is verdeeld in twee delen die beide strategieën behandelen.

Een algemene inleiding over het belang van dosisverificatieprocedures in de radiotherapie is te vinden in hoofdstuk 1, met een focus op het gebruik van 3D-verificatie in geavanceerde radiotherapie. In hoofdstuk $\mathbf{2}$ wordt een uitgebreid overzicht gegeven van het gebruik van elektronische röntgen camera's ("Electronic Portal Imaging Devices", EPID's) voor dosimetrie doeleinden. In deze literatuurstudie worden de dosimetrische eigenschappen en karakteristieken van EPID's en de kalibratiemethoden beschreven, evenals het gebruik van EPID's voor "pre-treatment" dosisverificatie en in vivo dosimetrie. Het type fouten dat kan worden opgespoord met EPID dosimetrie en veelvuldig gebruikte acceptatie- en afwijzingscriteria voor de interpretatie van de waargenomen verschillen tussen de geplande en de gemeten dosisverdelingen worden gepresenteerd, verder zijn de huidige klinische toepassingen van EPID dosimetrie samengevat.

De hoofdstukken 3-5 beschrijven verificatiemethoden en procedures die worden toegepast voordat de eerste fractie van een behandeling wordt gegeven. Deze "pretreatment" verificatie kan gebruikt worden voor het opsporen van fouten gemaakt tijdens het maken van een behandelplan, dosisberekening in de patiënt, overdracht van het behandelplan naar het bestralingstoestel of het opsporen van verschillen tussen de geplande en werkelijke geleverde energie-fluentieverdeling van de behandelvelden. Hoofdstuk 3 beschrijft het model in detail. Dit model meet de dosis afgeleverd door de lineaire versneller met een EPID en reconstrueert de 3D-dosisverdeling in een vooraf gedefinieerd fantoom met behulp van een Monte Carlo dosisberekening. De methode is geëvalueerd voor homogene fantomen waarbij een goede overeenkomst tussen 3D-gereconstrueerde en gemeten dosisdistributies is waargenomen; $99 \%$ van de punten waren binnen $3 \%$ of $3 \mathrm{~mm}$ van de gemeten dosisverdeling, zowel voor 3D-conformale en intensiteit-gemoduleerde bestralingsvelden.

In hoofdstuk 4 wordt het dosisreconstructiemodel uitgebreid voor situaties waarin weefsel-equivalente materialen en dichtheidsverschillen voorkomen waarbij dit is geverifieerd met behulp van diverse fantomen van verschillende afmetingen, met inbegrip van inhomogene en antropomorfe fantomen. Een vergelijking tussen het 
dosisreconstructiemodel en de 3D-dosisverdeling berekend met behulp van het eenvoudige convolutie en meer geavanceerde superpositie algoritme van het klinisch toegepaste behandelplanningssysteem ("treatment planning system", TPS) is uitgevoerd. Het convolutie algoritme is niet nauwkeurig in het berekenen van de dosis rond grensvlakken met verschillende dichtheden die aanwezig zijn bij de long en bij luchtholtes in het hoofd-hals gebied. De nauwkeurigheid van de dosisberekening van het superpositie algoritme geïmplementeerd in het TPS is in goede overeenkomst met het op Monte Carlo berekeningen gebaseerde dosisreconstructie algoritme, met uitzondering van enkele kleine regio's in botstructuren.

Hoofdstuk 5 beschrijft de toepassing van de methoden besproken in de hoofdstukken 3 en 4 in een klinische setting, en een evaluatie wordt gepresenteerd op basis van de "planning CT-scan" van de behandeling van patiënten met tumoren in het hoofd-hals en thorax gebied. De verschillen worden uitgedrukt in parameters van het dosis-volume histogram (DVH). In de thorax regio zijn de plannen berekend met behulp van het convolutie algoritme van het TPS vergeleken met de 3Dgereconstrueerde dosisverdeling waarbij gemiddeld $5 \%$ minder dosis in het doelgebied komt. Bovendien treedt er een verminderde dosishomogeniteit in het doelgebied op, aangegeven door de afname van de $D_{95 \%}$ parameter van gemiddeld $9 \%$. Herberekenen van deze plannen met behulp van het superpositie algoritme reduceerde deze verschillen tot minder dan $2 \%$. Hieruit kan worden geconcludeerd dat het superpositie algoritme, of meer geavanceerde algoritmes in het algemeen, nodig zijn voor de berekening van accurate dosisverdelingen in de thorax regio. Voor de patiënten met tumoren in het hoofd-hals gebied, behandeld met intensiteit-gemoduleerde radiotherapie ("Intensity-Modulated Radiation Therapy", IMRT), zijn de verschillen tussen de geplande dosisverdeling van het TPS gebruik makende van de superpositie algoritme en de 3D-gereconstrueerde dosisverdeling in het algemeen onder de 3\%. Voor de dosis in het doelvolume waren de $D_{5 \%}$, de gemiddelde dosis en de $D_{95 \%}$ binnen $2 \%$ van de geplande waarden en de dosiswaarden van de gezonde weefsels waren binnen $3 \%$ van de geplande waarden.

In hoofdstuk 6 worden de implicaties van het gebruik van nauwkeurige dosisberekening algoritmen voor het ontwerpen van 3D-conformal behandelplannen voor patiënten met niet-kleincellig longkanker besproken. Verschillende behandelplannen zijn ontworpen met gebruikmaking van zowel het convolutie en het superpositie algoritme gebaseerd op een dosis-escalatie protocol waarbij gezonde weefsels de beperkende factor vormen. Als van het convolutie dosisberekening algoritme in het TPS wordt overgestapt naar het superpositie algoritme, resulteert dit in zowel een lagere totale tumordosis en een afname van dekking van het doelvolume. In dit dosisescalatie protocol, waarbij de tumordosis wordt beperkt door gezonde weefsel complicaties, is een vergroting van de bestralingsvelden om voor een adequate tumordekking te zorgen niet mogelijk omdat dit tot een te grote toxiciteit van deze gezonde weefsels zal leiden. Daarom is een strategie bepaald die in staat is om bij de invoering van deze meer geavanceerde dosisberekening algoritmen toch tot een hogere totale tumor dosis komt door het enigszins verminderen van de tumordekking in bepaalde gebieden, waarbij wordt uitgegaan van een iso-toxische behandeling.

Het tweede deel van dit proefschrift behandelt verificatieprocedures die worden toegepast tijdens de behandeling. Voor dit doel is het noodzakelijk te beschikken over 
een nauwkeurig model van de anatomie van de patiënt voorafgaand aan, of vlak na een behandelsessie, waarbij de patiënt nog steeds in behandelpositie is. Hoofdstuk 7 beschrijft een methode voor het maken van een megavolt (MV) "cone-beam CT-scan" in de behandelkamer en een kalibratie-procedure is ontwikkeld voor het gebruik van deze beelden voor dosisberekeningen. De beelden worden gecorrigeerd voor de zgn "cupping" artefacten en de Hounsfield-eenheden worden omgezet in elektronendichtheidswaarden. Dit maakt deze beelden geschikt voor dosisberekening met behulp van het TPS of Monte Carlo dosisberekeningen.

Hoofdstuk 8 combineert de methoden ontwikkeld voor de "pre-treatment" verificatieprocedure met MV "cone-beam" CT-beeldvorming. De werkelijk afgegeven bestralingsvelden worden gemeten achter de patiënt met de EPID en de informatie in deze velden wordt gebruikt in combinatie met de "cone-beam CT-scan" gemaakt in de bestralingsruimte. Een terugprojectie-model is ontwikkeld dat de transmissie EPID beelden achter een patiënt tijdens de behandeling gebruikt voor het reconstrueren van de 3D-dosisverdeling in de werkelijke patiëntanatomie verworven met behulp van de MV "cone-beam CT-scan". De nauwkeurigheid van dit model werd beoordeeld met behulp van een fantoomstudie waarbij de fout in de dosis kleiner was dan $3 \%$ voor de het grootste deel van de punten in de 3D-dosisverdeling. Omdat verschillen kunnen worden gekwantificeerd in de werkelijke patiëntanatomie, is kwantificering van de dosis in het doelgebied en in de gezonde weefsels mogelijk. Deze 3Ddosisreconstructiemethode is daarom een ideale verificatieprocedure omdat in één keer grote delen van het radiotherapieproces gecontroleerd worden zoals de dosisafgifte, dosisberekening, patiëntpositionering en veranderingen in de anatomie van de patiënt ten opzichte van de planningssituatie. Als "proof-of-principle" is de methode gebruikt om de afgegeven dosis te controleren voor een patiënt met een tumor in het hoofd-hals gebied behandeld met IMRT. Behalve enkele kleine regio's rond de luchtholtes, was er een goede overeenkomst tussen de gereconstrueerde dosisverdeling in de MV "cone-beam CT-scan" en de geplande dosisverdeling in de "planning CT-scan". De DVH's voor de gezonde weefsels lagen binnen 3\% van de geplande waardes en de gereconstrueerde gemiddelde dosis in het doelvolume week niet meer dan $2 \%$ van de geplande waarde af.

Hoofdstuk 9 beschrijft een algemene discussie over het gebruik van 3D "pretreatment" verificatie en 3D in vivo dosimetrie methoden voor de verificatie van geavanceerde radiotherapie. Deze modellen zijn nodig voor uitbreiding in het kader van adaptieve radiotherapie ("adaptive radiation therapy", ART) om ook de afgegeven dosis tijdens de behandeling mee te nemen: dosis-gestuurde radiotherapie ("doseguided radiation therapy", DGRT). Toekomstperspectieven zijn beschreven en een visie is gegeven op het op een adaptieve wijze implementeren van deze geavanceerde modellen tijdens een radiotherapeutische behandeling. 

LIST OF PUBLICATIONS 



\section{PUBLICATIONS}

Published articles

W.J.C. van Elmpt, S.M.J.J.G. Nijsten, B.J. Mijnheer and A.W.H. Minken, "Experimental verification of a portal dose prediction model," Medical Physics 32(9): 2805-2818 (2005).

W.J.C. van Elmpt, T.M. Nijsen, P.A. Griep, J.B. Arends, "A model of heart rate changes to detect seizures in severe epilepsy," Seizure 15(6): 366-375 (2006).

W.J.C. van Elmpt, S.M.J.J.G. Nijsten, R.F.H. Schiffeleers, A.L.A.J. Dekker, B.J. Mijnheer, P. Lambin and A.W.H. Minken, "A Monte Carlo based three-dimensional dose reconstruction method derived from portal dose images," Medical Physics 33(7): 2426-2434 (2006).

W.J.C. van Elmpt, S.M.J.J.G. Nijsten, A.L.A.J. Dekker, B.J. Mijnheer and P. Lambin, "Treatment verification in the presence of inhomogeneities using EPID-based threedimensional dose reconstruction," Medical Physics 34(7): 2816-2826 (2007).

S.M.J.J.G. Nijsten, W.J.C. van Elmpt, M. Jacobs, B.J. Mijnheer, A.L.A.J. Dekker, P. Lambin and A.W.H. Minken, "A global correction model of a-Si EPIDs used for transit dosimetry," Medical Physics 34(10): 3872-3884 (2007).

W.J.C. van Elmpt, S.M.J.J.G. Nijsten, A.L.A.J. Dekker, B.J. Mijnheer and P. Lambin, "The next step in patient specific QA: 3D dose verification for conformal and intensitymodulated RT based on EPID dosimetry and Monte Carlo dose calculations," Radiotherapy and Oncology 86(1):86-92 (2008).

S.F. Petit, W.J.C. van Elmpt, S.M.J.J.G. Nijsten, A.L.A.J. Dekker and P. Lambin, "Calibration of megavoltage cone-beam CT for radiotherapy dose calculations: correction of cupping artifacts and conversion of CT-numbers to electron density," Medical Physics 35(3):849-865 (2008).

W.J.C. van Elmpt, M.C. Öllers, M. Velders, K. Poels, B.J. Mijnheer, D. De Ruysscher, A.L.A.J. Dekker, P. Lambin and L. Boersma, "Transition from a simple to a more advanced dose calculation algorithm for radiotherapy of non-small cell lung cancer (NSCLC): Implications for clinical implementation in an individualized dose-escalation protocol," Radiotherapy and Oncology 88(3):326-334 (2008).

W.J.C. van Elmpt, L.N. McDermott, S.M.J.J.G Nijsten, M. Wendling, P. Lambin and B.J. Mijnheer, "A literature review of electronic portal imaging for radiotherapy dosimetry", Radiotherapy and Oncology 88(3):289-309 (2008). 
S.M.J.J.G. Nijsten, W.J.C. van Elmpt, B.J. Mijnheer, A.W.H. Minken, L.C.G.G. Persoon, P. Lambin and A.L.A.J. Dekker, "Prediction of DVH parameter changes due to set-up errors for breast cancer treatment based on 2D portal dosimetry", Medical Physics 36(1):8394 (2009).

W.J.C. van Elmpt, S.M.J.J.G. Nijsten, S.F. Petit, B.J. Mijnheer, P. Lambin and A.L.A.J. Dekker, "3D in vivo dosimetry using megavoltage cone-beam CT and EPID dosimetry," International Journal of Radiation Oncology*Biology*Physics (in press) (2009).

\section{Published abstracts}

W.J.C. van Elmpt, S.M.J.J.G. Nijsten, B.J. Mijnheer, A.W.H. Minken. "Accuracy of primary portal dose extraction for in-vivo dosimetry purposes," ESTRO 2004 conference, Amsterdam, The Netherlands, Radiotherapy and Oncology 73(Suppl 1):S114-115 (2004).

W.J.C. van Elmpt, S.M.J.J.G. Nijsten, R.F.H. Schiffeleers, A.L.A.J. Dekker, B.J. Mijnheer, P. Lambin and A.W.H. Minken, "Three-Dimensional Dose Reconstruction for IMRT PreTreatment Verification using Portal Dose Images," the 9th International Workshop on Electronic Portal Imaging (EPI2k6), 10th -12th April 2006, Melbourne, Australia.

S.M.J.J.G. Nijsten, W.J.C. van Elmpt, B.J. Mijnheer, P. Lambin and A.W.H. Minken, "A correction model for spectral response of an a-Si EPID during exit-dosimetry," the 9th International Workshop on Electronic Portal Imaging (EPI2k6), 10th -12th April 2006, Melbourne, Australia.

W.J.C. van Elmpt, S.M.J.J.G. Nijsten, B.J. Mijnheer, A.L.A.J. Dekker and P. Lambin, "PreTreatment verification using $3 D$ dose reconstruction based on EPID measurements," ESTRO 2006 conference, Leipzig, Germany. Radiotherapy and Oncology 81(Suppl 1):S388 (2006).

S.F. Petit, W.J.C. van Elmpt, S.M.J.J.G. Nijsten, A.L.A.J. Dekker and P. Lambin, "Various procedures for the reduction of cupping artifacts in MegaVoltage Cone-Beam CT," ESTRO 2006 conference, Leipzig, Germany. Radiotherapy and Oncology 81(Suppl 1):S561 (2006).

W.J.C. van Elmpt, S.M.J.J.G. Nijsten, B.J. Mijnheer, A.L.A.J. Dekker and P. Lambin, "Treatment verification using 3-D dose reconstruction based on EPID measurements," Belgian Hospital Physicist Association (BHPA), Liège, Belgium (2007).

S.F. Petit, W.J.C. van Elmpt, S.M.J.J.G. Nijsten, A.L.A.J. Dekker and P. Lambin, "The use of Mega Volt Cone Beam CT for Dose Calculations," Belgian Hospital Physicist Association (BHPA), Liège, Belgium (2007). 
W.J.C. van Elmpt, S.M.J.J.G. Nijsten, B.J. Mijnheer, A.L.A.J. Dekker and P. Lambin, "The next step in patient-specific $Q A: 3 D$ dose verification for conformal and intensitymodulated RT based on EPID dosimetry and Monte Carlo dose calculations," ESTRO physics 2007 conference, Barcelona, Spain. Radiotherapy and Oncology 84(Suppl 1):S96 (2007).

S.M.J.J.G. Nijsten, W.J.C. van Elmpt, B.J. Mijnheer, A.L.A.J. Dekker, P. Lambin and A.W.H. Minken, "An energy spectrum response correction model for a-Si EPIDs used for transit dosimetry," ESTRO physics 2007 conference, Barcelona, Spain. Radiotherapy and Oncology 84(Suppl 1):S162 (2007).

W.J.C van Elmpt, M.C. Öllers, M. Velders, K. Poels, B.J. Mijnheer, D. De Ruysscher, A.L.A.J. Dekker, P. Lambin and L. Boersma, "Transition from a simple to an advanced dose calculation algorithm for radiotherapy of non-small cell lung cancer (NSCLC): Implications for implementation in a clinical individualized dose-escalation protocol," ESTRO physics 2007 conference, Barcelona, Spain. Radiotherapy and Oncology 84(Suppl 1):S199 (2007).

W.J.C. van Elmpt, S.M.J.J.G. Nijsten, B.J. Mijnheer, A.L.A.J. Dekker and P. Lambin, "The next step in patient-specific QA: $3 D$ dose verification for conformal and intensitymodulated radiotherapy based on EPID dosimetry and MC dose calculations," ASTRO annual meeting 2007, Los Angeles, USA. International Journal of Radiation Oncology*Biology*Physics 69(3), Suppl 1: S672-S673 (2007).

W.J.C. van Elmpt, S.F. Petit, A.L.A.J. Dekker and P. Lambin, "Voxel Dose Probability: A New Concept for Treatment Planning Incorporating Dose Uncertainties during Treatment Delivery," ASTRO annual meeting 2007, Los Angeles, USA. International Journal of Radiation Oncology*Biology*Physics 69(3) ;(Suppl 1):S696 (2007).

W.J.C. van Elmpt, S.F. Petit, S.M.J.J.G. Nijsten, B.J. Mijnheer, P. Lambin and A.L.A.J. Dekker, "3D in-vivo dose reconstruction using EPID measurements and MegaVoltage cone-beam CT scans," the 10th International Workshop on Electronic Portal Imaging (EPI2k8), 20th -22th May 2008, San Francisco, USA.

S.M.J.J.G. Nijsten, W.J.C. van Elmpt, R. Kollaard, L.C.G.G. Persoon, B.J. Mijnheer, A.L.A.J. Dekker and P. Lambin, "Routine 2-D in vivo verification using EPID dosimetry: One year clinical experience at MAASTRO," the 10th International Workshop on Electronic Portal Imaging (EPI2k8), 20th -22th May 2008, San Francisco, USA.

S.M.J.J.G. Nijsten, W.J.C. van Elmpt, B.J. Mijnheer, L.C.G.G. Persoon, P. Lambin and A.L.A.J. Dekker, " $2 D$ in-vivo verification in clinical routine using EPID dosimetry: clinical experience with more than 2000 patients," ESTRO 2008 conference, Göteborg, Sweden. Radiotherapy and Oncology 88(Suppl 2):S159 (2008). 
W.J.C. van Elmpt, S.M.J.J.G. Nijsten, S.F. Petit, B.J. Mijnheer, P. Lambin and A.L.A.J. Dekker, "A method to perform $3 D$ in vivo dosimetry using MV cone-beam CT and EPID dosimetry: from planned to measured dose distribution," ESTRO 2008 conference, Göteborg, Sweden. Radiotherapy and Oncology 88(Suppl 2):S160-161 (2008).

P. Lambin, S.F. Petit, H. Aerts, W.J.C. van Elmpt, S. Fekkes, B. Wouters, A. Dekker and D. De Ruysscher, "Radiotherapy 5.0 - Voxel control probability guided radiotherapy (VCP-GRT): an attempt to integrate molecular imaging and dose uncertainties with modern radiation dose delivery," ESTRO 2008 conference, Göteborg, Sweden. Radiotherapy and Oncology 88(Suppl 2):S300-301 (2008).

S. Fekkes, W.J.C. van Elmpt, J. Orban de Xivry, G. Janssens, P. Lambin and A.L.A.J. Dekker, "Accuracy assessment of inter-fraction dose accumulation using non-rigid deformation models: study in cubic silicon phantoms," ESTRO 2008 conference, Göteborg, Sweden. Radiotherapy and Oncology 88(Suppl 2):S363 (2008). 
DANKWOORD 



\section{DANKWOORD}

Als eerste wil ik mijn promotor prof. Philippe Lambin en copromotoren dr. ir. André Dekker en dr. Ben Mijnheer bedanken voor hun bijdrage en ondersteuning bij mijn promotie.

Beste Philippe, het viel mij altijd op dat, hoewel we niet vaak overleg hadden, het toch altijd weer interessante bijeenkomsten waren, waaruit altijd genoeg inspiratie en ideeën voortkwamen voor de komende maanden. Het review-artikel bijvoorbeeld was een van die ideeën die precies op het juiste moment kwam, zowel voor mijn promotieonderzoek, maar ook in het vakgebied was de tijd gekomen om alle relevante literatuur op een rijtje te zetten.

Beste André, jij hebt nadat ik al een jaar gestart was de begeleiding op je genomen. Jouw gestructureerde manier van werken en analyseren van problemen is een van de eigenschappen waar ik tijdens mijn promotie soms aan moest wennen maar uiteindelijk het werk makkelijker maakte.

Beste Ben, ik heb bewondering voor de snelheid en nauwgezetheid waarmee je mijn artikelen, vaak in een zeer prematuur stadium, al wilde nalezen en corrigeren. Zeker voor de eerste artikelen die we samen geschreven hebben was dit een grote hulp waar ik nu bij het schrijven van een willekeurig artikel, projectvoorstel of wetenschappelijk abstract nog steeds profijt van heb.

Beste Bas, eerst als begeleider tijdens mijn afstuderen, later als mede-onderzoeker op de dagen dat je aan je promotie werkte, en nu ook als paranimf. De lange meetsessies die we gehouden hebben de afgelopen jaren waren, ondanks het late tijdstip waarop we meestal klaar waren, zeker gezellig. Als ik nu terugkijk op wat wij samen de afgelopen jaren bereikt hebben, denk ik dat we trots mogen zijn op het verzette werk. Hoewel we beiden samen op 'lijn 16' de route uitstippelden, zullen we waarschijnlijk in de toekomst onze eigen bestemming kiezen, maar de eindbestemming die we samen voor ogen hadden hebben we naar mijn mening zeker gehaald. Succes met het afronden van jouw 'boekje'.

Verder zou ik graag de studenten die hebben meegeholpen en de co-auteurs van de verschillende artikelen die de basis vormen van dit proefschrift willen bedanken: Leah, Markus, Rob, Chris, Stein, Michel, Kim, Marije, Dirk, Liesbeth en Steven. Jullie bijdragen zijn zeer waardevol geweest bij het tot stand komen van de verschillende hoofdstukken van dit proefschrift.

De mensen van de TiQc, en in het bijzonder Joep, Sem, Jonathan en Davy. De talloze malen dat jullie mij hebben geholpen bij het opzetten van de waterbak, ontwikkelen van de filmmetingen of het verhelpen van voor mij totaal onbegrijpelijke foutmeldingen van de versneller. Zonder jullie hulp was het allemaal niet zo snel verlopen en een groot deel van de meetresultaten in dit proefschrift zijn mede dankzij jullie verkregen.

Al mijn kamergenoten die ik in de loop van de jaren gehad heb. Het begon eigenlijk al in Heerlen, waar we buiten in een 'keet' op de parkeerplaats als gevolg van de dunne wandjes tussen de verschillende kamers eigenlijk allemaal op 1 grote kamer zaten. Een speciaal woord van dank aan André Minken; bedankt dat je mij de mogelijkheid hebt gegeven om te starten met dit promotie-onderzoek, helaas hebben we het niet samen kunnen afmaken, maar een groot deel van dit onderzoek hebben we 
samen uitgezet en terugkijkend hierop is het grootste deel ervan uitgekomen! De klinisch fysici en klifio's bij wie ik altijd wel terecht kon voor klinisch of patiënt georiënteerde vragen; deze setting heeft mij zeer geholpen om mijn onderzoek in een groter geheel te doen bekijken. En verder al mijn andere kamergenoten die de afgelopen 4 jaar met mij opgescheept hebben gezeten: Erik, Hugo, Esther, Sonia, Lucas, Steven, Marco, Ramon, Miguel, Katia en Ruud. Ik hoop dat ik nu nog steeds mee mag met jullie promovendi-tapas-etentjes.

Verder zou ik ook de leden van de beoordelingscommissie, prof. Wildberger, prof. Heijmen, prof. Ter Haar Romeny, dr. Verhaegen en prof. De Wagter willen bedanken voor het grondig bestuderen van dit proefschrift. Rianne, bedankt voor de secretariële ondersteuning bij de voltooiing van dit proefschrift.

Als laatste mijn lieve vriendin en familie, jullie hadden altijd vragen over mijn werk en interesse in mijn promotieonderzoek waarbij mijn uitleg misschien niet altijd even uitgebreid of duidelijk was, maar ik hoop dat jullie na vandaag toch een goed beeld hebben kunnen vormen van wat ik de afgelopen jaren heb gedaan. Bedankt voor jullie steun. 
CURRICULUM VITAE 



\section{CURRICULUM VITAE}

Wouter van Elmpt werd geboren op 6 juni 1981 in Elsloo, Limburg. Hij voltooide in 1999 het VWO op scholengemeenschap Groenewald in Stein en vervolgde zijn studie met een opleiding Technische Natuurkunde aan de Technische Universiteit in Eindhoven (TU/e). Hier kwam hij tot de ontdekking dat de techniek in de gezondheidszorg goed bij zijn interesses aansloot en koos hij voor keuzevakken gericht op de medische fysica. Na een stage bij epilepsiecentrum Kempenhaeghe in Heeze kwam hij bij MAASTRO clinic terecht, toen nog gevestigd te Heerlen. Hij voltooide zijn afstudeeropdracht en rondde hiermee in oktober 2004 de ingenieursopleiding aan de TU/e af. Na een korte tijd te hebben gezocht naar een geschikte promotieplaats, vond hij deze snel op dezelfde werkplek en startte hij 1 april 2005 met zijn promotieonderzoek binnen de Klinische Fysica Groep van MAASTRO clinic. Op 1 december 2008 is hij gestart als postdoc onderzoeker bij de vakgroep radiotherapie van de Universiteit Maastricht met als onderwerp beeldvorming en adaptieve radiotherapie bij longkankerpatiënten. 


